\title{
Advanced Gas Cooling Study for the Hospital at Davis-Monthan AFB, AZ
}

by

Michael A. Caponegro

William T. Brown III

Timothy W. Pedersen

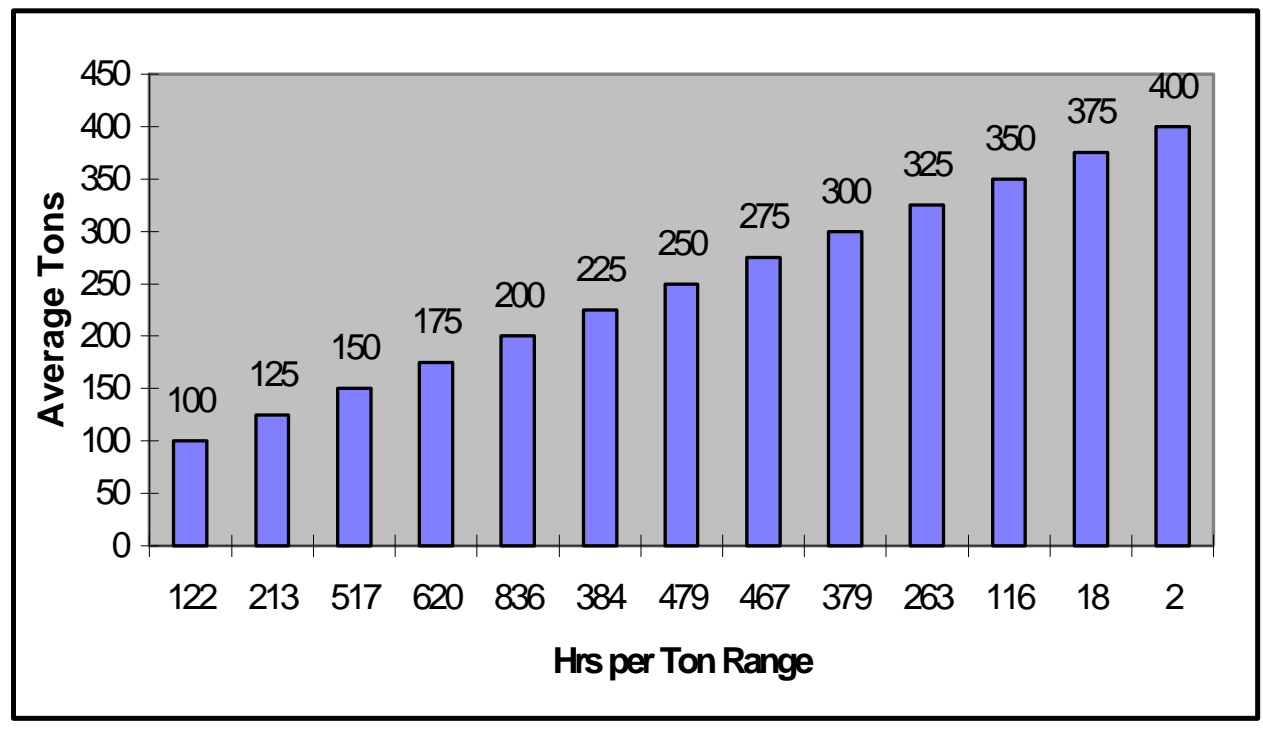

Based on its experience with a cogeneration project at Tyndall AFB, the Air Force Civil Engineering Support Agency (AFCESA) tasked the U.S. Army Construction Engineering Research Laboratories (USACERL) to perform an analysis to see if such a concept, or some other cooling options, could be of economic benefit at the Air Force medical facility at Davis-Monthan AFB, AZ, where the cost of purchased electrical power is relatively high compared to that of natural gas.

USACERL researchers developed a cooling load profile for the facility by reviewing plant records and interviewing plant operators. Boiler logs (daily and monthly) were consulted to determine heating loads, and a spreadsheet was developed to analyze nine options. Savings and first costs were input into the Life Cycle Cost in Design (LCCID) computer program to determine simple paybacks and savings-to-investment ratios for all options. Based on the results of the investigation, preferred options were recommended for meeting the facility cooling load. 


\section{Foreword}

This study was conducted for the Air Force Civil Engineering Support Agency (AFCESA) under Military Interdepartmental Purchase Request (MIPR) No. N94-92, Work Unit WL4, "Evaluation and Application of Gas Cooling Technologies." The technical monitor was Rich Bauman, AFCESA.

The work was performed by the Utilities Division (UL-U) of the Utilities and Industrial Operations Laboratory (UL), U.S. Army Construction Engineering Research Laboratories (CERL). The CERL Principal Investigator was Timothy W. Pedersen. Martin J. Savoie is Chief, CECER-UL-U, and Dr. J ohn Bandy is Operations Chief, CECER-UL. The responsible Technical Director was Gary W. Schanche, CECER-TD. The CERL technical editor was William J. Wolfe, Technical Information Team.

Dr. Michael J . O'Connor is Director of CERL. 


\section{Contents}

Foreword

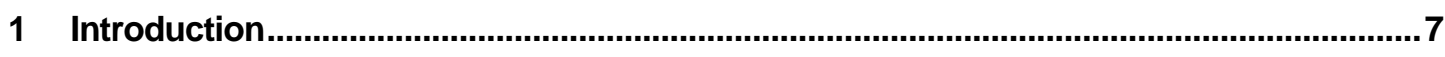

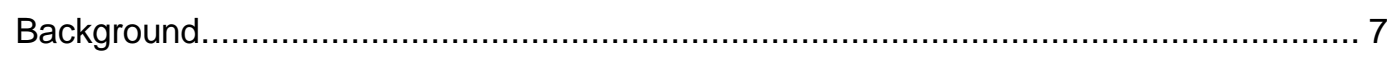

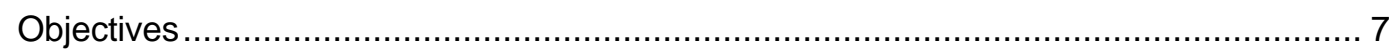

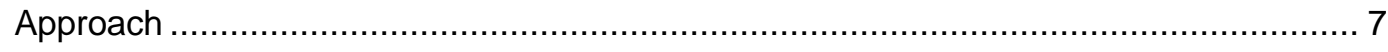

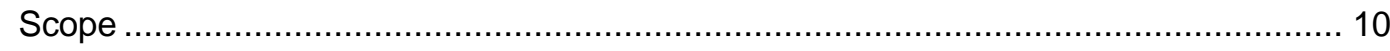

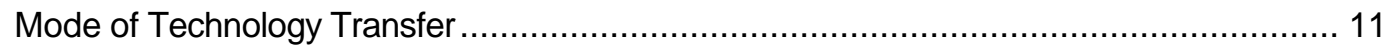

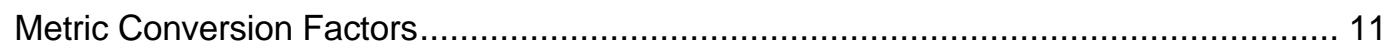

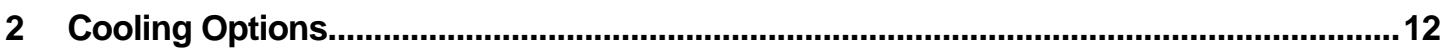

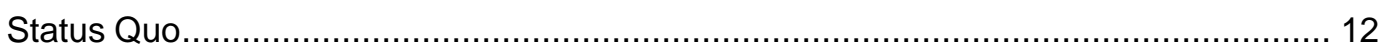

Two Hundred Fifty (250)-Ton Capacity Natural Gas Engine-Driven Chiller With Heat Recovery (Option \#1) ...................................................................... 12

Power Generation With Sufficient Waste Heat To Operate a Water Chilling Unit ............ 13

Power Generation With Sufficient Waste Heat To Operate a Water Chilling Unit and Satisfy Facility Thermal Requirements.............................................................. 15

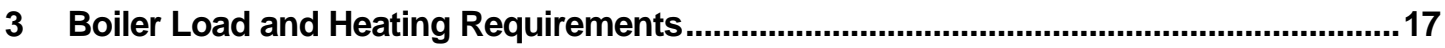

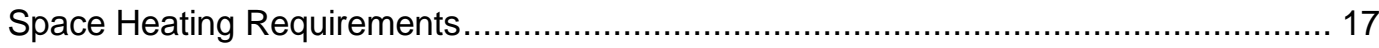

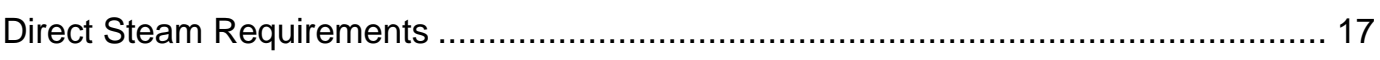

Domestic Hot Water Heating Requirements................................................... 17

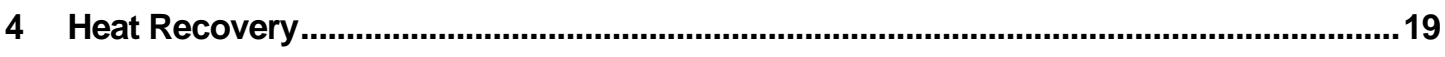

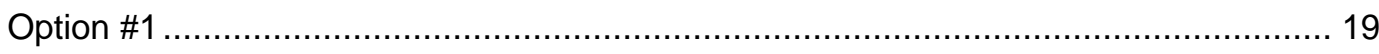

Remaining Options (Heat Recovery from Engine-Generator Sets) ........................... 19

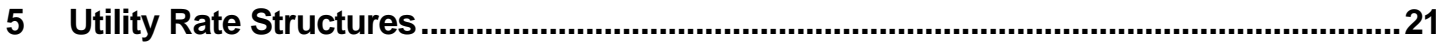

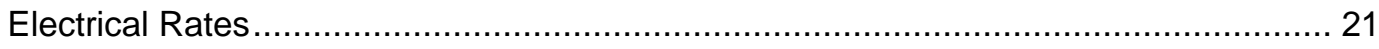

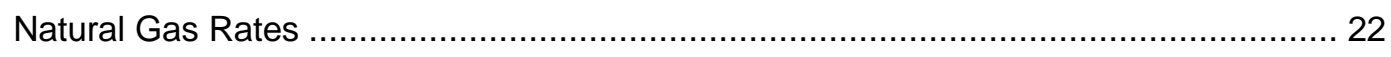

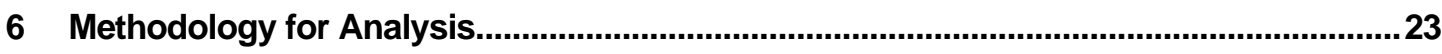

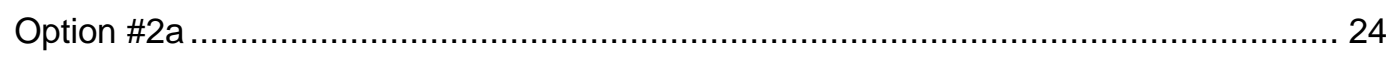

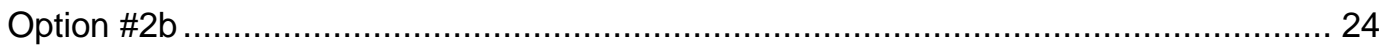

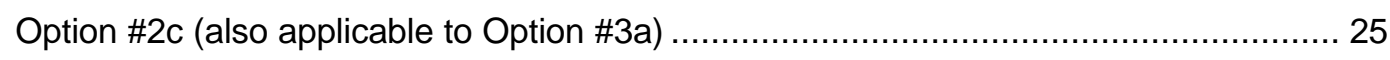




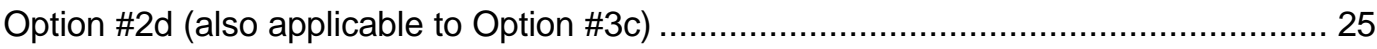

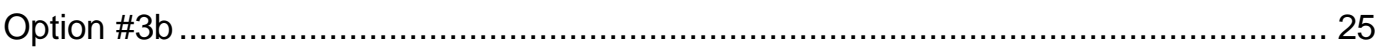

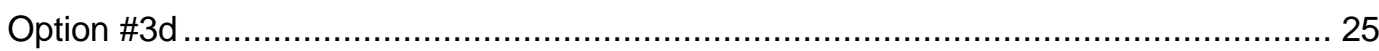

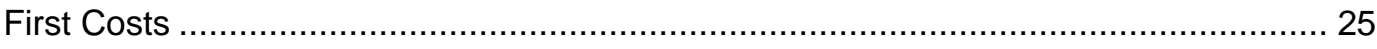

Life Cycle Cost Analysis ............................................................................... 26

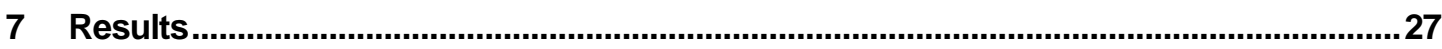

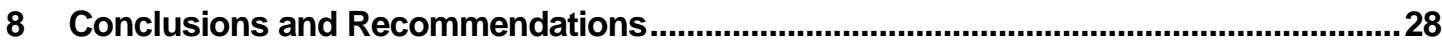

Appendix A: Notes Regarding Interviews and Discussions for Chiller Study at

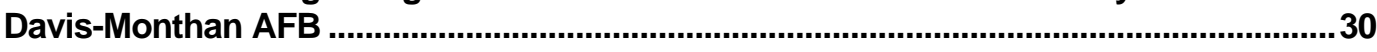

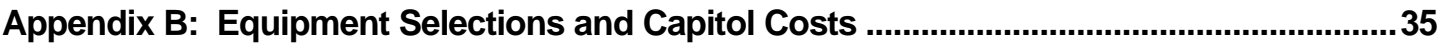

Appendix C: Recent Electric Bills for Davis-Monthan AFB ...........................................39

Appendix D: Spreadsheet Calculation Based on Option \#2a .................................................53

Appendix E: Spreadsheet Calculation Based on Option \#2b..........................................54

Appendix F: Spreadsheet Calculation Based on Options \#2c and 3a................................55

Appendix G: Spreadsheet Calculation Based on Options \#2d and 3c...............................56

Appendix H: Spreadsheet Calculation Based on Option \#3b............................................57

Appendix I: Spreadsheet Calculation Based on Option \#3d .............................................58

Appendix J: Construction Cost Estimates for Each Option ................................................59

Appendix K: Energy Cost Estimates for Each Option...........................................................69

Appendix L: Calculated Paybacks and Savings-to-Investment Ratio for Option 1 ............129

Appendix M: Calculated Paybacks and Savings-to-Investment Ratio for Option 2a .........130

Appendix N: Calculated Paybacks and Savings-to-Investment Ratio for Option 2b .........132

Appendix O: Calculated Paybacks and Savings-to-Investment Ratio for Option 2c .........134

Appendix P: Calculated Paybacks and Savings-to-Investment Ratio for Option 2d...........136

Appendix Q: Calculated Paybacks and Savings-to-Investment Ratio for Option 3a .........138

Appendix R: Calculated Paybacks and Savings-to-Investment Ratio for Option 3b .........140 
Appendix S: Calculated Paybacks and Savings-to-Investment Ratio for Option 3c..........142 Appendix T: Calculated Paybacks and Savings-to-Investment Ratio for Option 3d ...........144 



\section{Introduction}

\section{Background}

The Air Force Civil Engineering Support Agency (AFCESA) has been actively involved with a cogeneration project at Tyndall AFB hospital. The project uses an absorption chiller to satisfy the hospital's base cooling load. The steam for activating the absorption chiller is obtained from waste heat, which is derived from an engine driving a generator to produce electrical power - which is also used by the hospital. Based on this experience, AFCESA funded USACERL to perform an analysis to see if such a concept, or some other cooling options, could be of economic benefit at the Air Force medical facility at Davis-Monthan AFB, $A Z$, where the cost of purchased electrical power is relatively high compared to that of natural gas. USACERL researchers evaluated the case of power generation providing sufficient waste heat to meet the facility base cooling load, and also considered options under which sufficient waste heat could be derived from power production so that a 250-ton absorption chiller could replace an existing motor-driven centrifugal chiller of equal capacity. (The centrifugal chiller uses a chlorofluorocarbon [CFC] refrigerant, R-11.) Heat produced as a result of power generation can be used to satisfy facility thermal, as well as cooling, loads.

\section{Objectives}

The objective of the study was to determine the approach that will minimize the cost of meeting the cooling requirements of the medical facility at DavisMonthan AFB.

\section{Approach}

\section{Cooling Load Profile}

Considerable time was spent developing a cooling load profile for the facility. This was done by meticulously reviewing plant records and discussing plant operation with the operators (Appendix A). Where data appeared inconsistent or 
erroneous, or was missing, trends were examined and reasonable estimates made for the actual cooling loads. Figures 1 and 2 show the results of the data analysis.

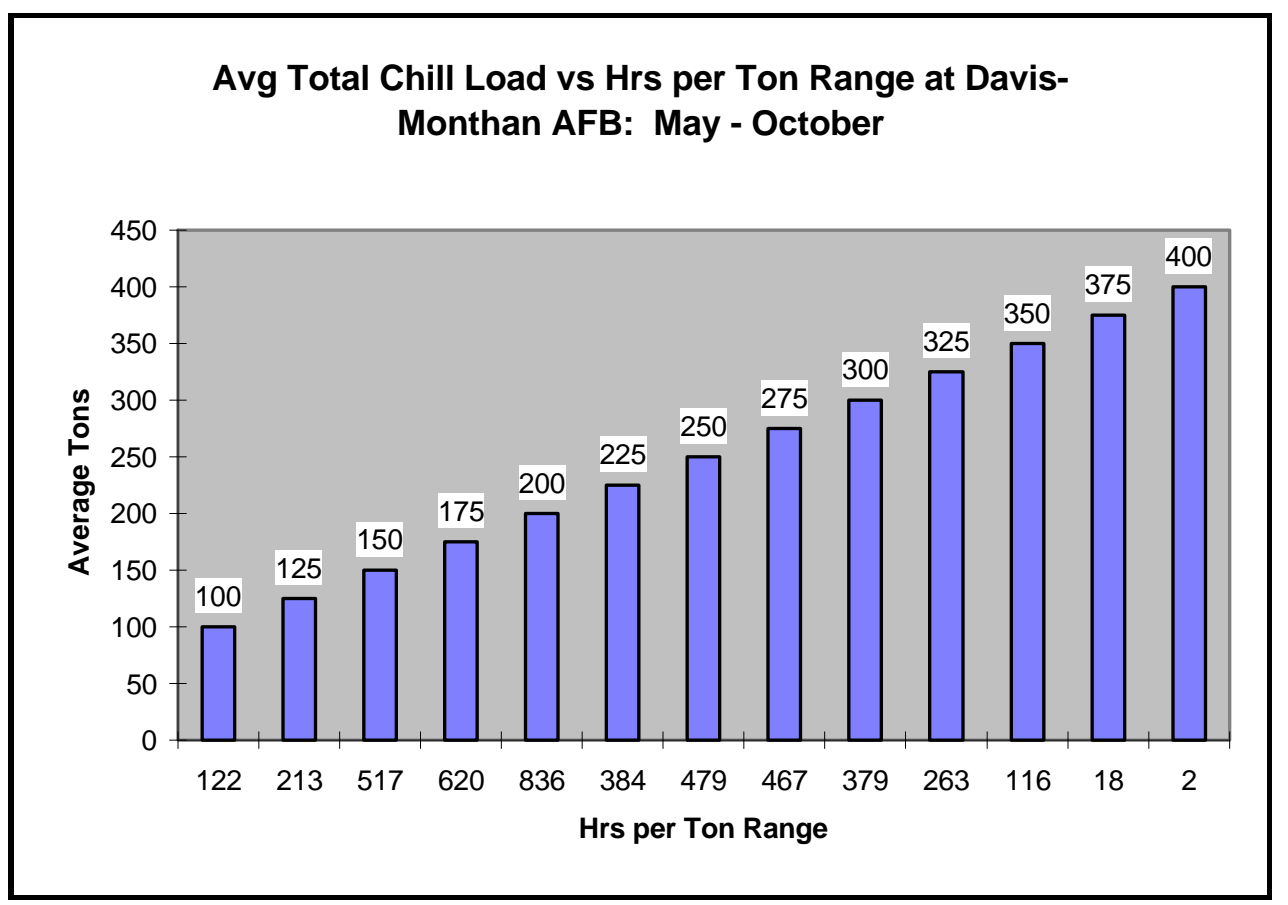

Figure 1. Cooling load estimate, May-October 1996.

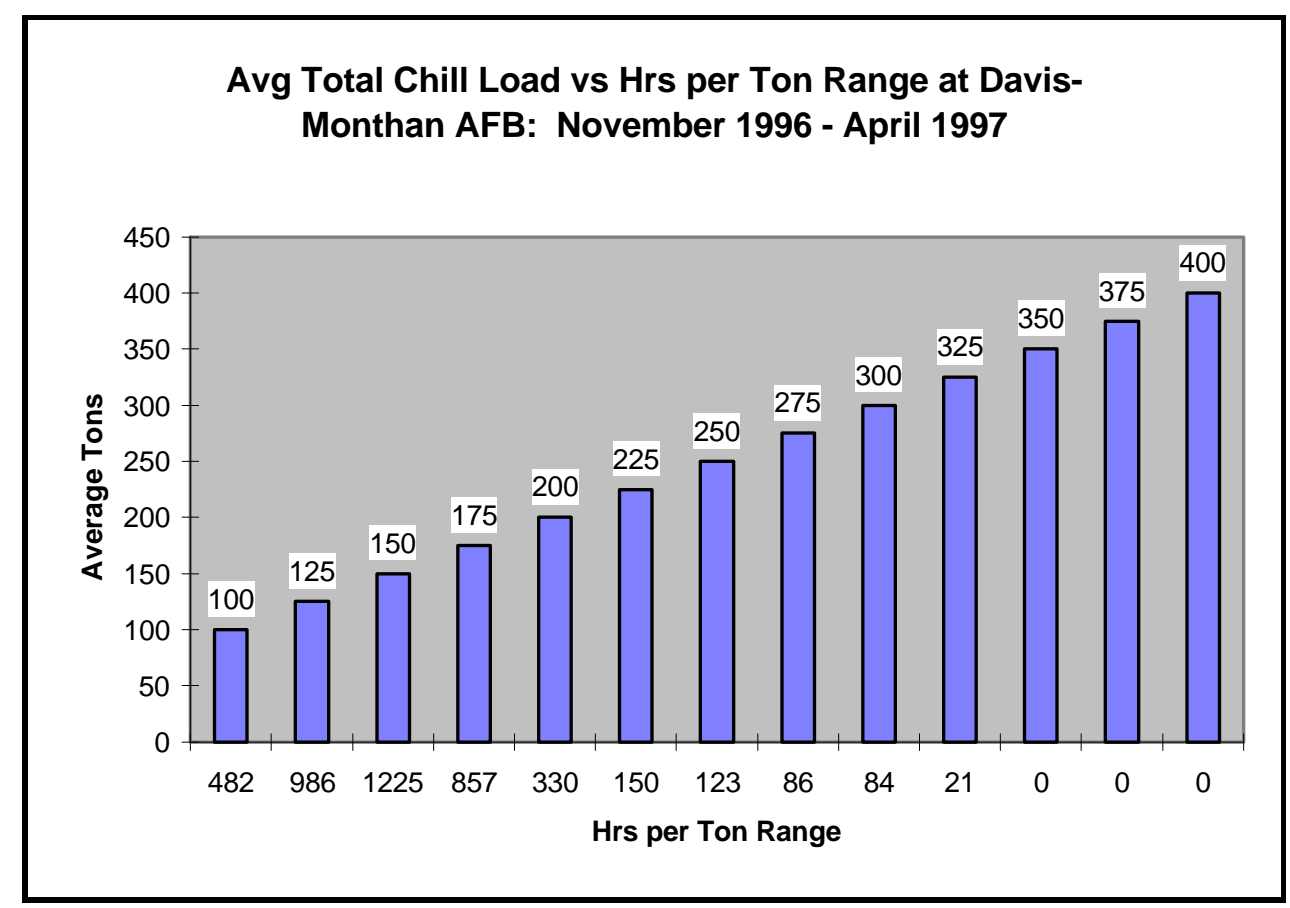

Figure 2. Cooling load estimate, November 1996-April 1997. 


\section{Boiler Load Profile}

Boiler logs (daily and monthly) were consulted to determine heating loads. The hospital cooling and heating plant was visited a number of times to obtain information on the plant equipment, including water chillers, cooling towers, boilers, pumps, and heat exchangers. It became obvious that the plant's boilers are grossly oversized for the load and, based on the pressure of the steam produced (50 psig>15 psig), that the boilers require onsite plant operators. Consequently, it became clear that waste heat from power generation could be used not only to meet facility cooling loads through absorption water chilling, but also to meet facility thermal loads, and in the process to possibly reduce manpower requirements and increase utility cost savings.

\section{Analysis}

An $E X C E L^{\circledR}$ spreadsheet was developed to analyze the numerous options that were considered. The options considered were:

- (Option \#1) Natural gas engine-driven chiller to replace existing 250-ton motor-driven centrifugal chiller, with waste heat used to offset facility thermal requirements

- (Option \#2a) Natural gas-fired engine-generator set, waste heat used to provide steam for single-effect absorption water chiller to meet facility base cooling load (100 tons) with residual heat used for thermal requirement

- (Option \#2b) Natural gas-fired engine-generator set, waste heat used to provide steam for double-effect absorption water chiller to meet facility base cooling load (100 tons), with residual heat used for thermal requirements

- (Option \#2c) Natural gas-fired engine-generator set, waste heat used to provide steam for single-effect absorption water chiller to replace existing 250-ton motor-driven centrifugal chiller, with residual heat used for thermal requirements

- (Option \#2d) Natural gas-fired engine-generator set, waste heat used to provide steam for double-effect absorption water chiller to replace existing 250-ton motor-driven centrifugal chiller, with residual heat used for thermal requirements

- (Option \#3a) Natural gas-fired engine-generator set, waste heat used to provide steam for single-effect absorption water chiller to meet facility base cooling load (100 tons) with residual heat adequate to satisfy facility thermal requirements (existing boilers as backup only)

- (Option \#3b) Natural gas-fired engine-generator set, waste heat used to provide steam for double-effect absorption water chiller to meet facility base cooling load (100 tons) with residual heat adequate to satisfy facility thermal requirements (existing boilers as backup only) 
- (Option \#3c) Natural gas-fired engine-generator set, waste heat used to provide steam for single-effect absorption water chiller to replace existing 250-ton motor-driven centrifugal chiller, with residual heat adequate to satisfy facility thermal requirements (existing boilers as backup only)

- (Option \#3d) Natural gas-fired engine-generator set, waste heat used to provide steam for double-effect absorption water chiller to replace existing 250-ton motor-driven centrifugal chiller, with residual heat adequate to satisfy facility thermal requirements (existing boilers as backup only).

In essence, Option \#1 did not entail the use of an engine-generator set, only a replacement of the existing 250-ton electric motor-driven centrifugal chiller with a natural gas engine-driven chiller, waste heat from which would partially offset facility thermal loads. Options $\# 2 a, 2 b, 2 c$, and $2 d$ entailed use of an enginegenerator set, with the waste heat providing sufficient energy to activate the absorption chiller and also to meet a portion of the facility thermal load. Options $\# 3 a, 3 b, 3 c$, and $3 d$ entailed use of an engine-generator set sized so that the waste heat would not only be sufficient to activate the absorption water chiller, but also to meet the entire thermal load of the facility. Note that, at one time, another option was considered for analysis: use of a 250-ton capacity, directfired, double-effect, absorption chiller. This option was discarded prior to more in-depth analysis because the coefficient-of-performance would be less than for the engine-driven option, particularly when heat is recovered from the engine.

Once the spreadsheet calculated the operating cost savings for each option, the savings and first costs were input into the Life Cycle Cost in Design (LCCID) computer program to determine simple paybacks and savings-to-investment ratios for all options. Based on the results of the investigation, recommendations were made as to the preferred option for meeting the facility cooling load.

\section{Scope}

The scope of the project was to investigate feasible options for meeting the facility's cooling loads, with emphasis on using waste heat from electrical power generation for absorption cooling. An ancillary benefit was that waste heat could be used not only for absorption cooling, but also for partially or totally meeting the medical facility thermal loads. 


\section{Mode of Technology Transfer}

This report documents the opportunities available and financial resources required for reducing cooling and overall utility costs for the Davis-Monthan AFB medical facility. USACERL would be amenable to developing a scope of work for architect-engineer (A-E) services to design the installation of the equipment for whatever option USAF management desires to pursue to reduce the medical facility's utility costs. Additionally, USACERL would be amenable to reviewing the design and participating in technical oversight during construction, as well as monitoring equipment performance to verify estimated savings. Additionally, the technologies considered here for possible application at the medical facility at Davis-Monthan AFB have the potential to benefit other DOD medical facilities. Consequently, it is recommended that this document be circulated to the larger DOD medical community for its consideration in applying the technologies at other sites.

\section{Metric Conversion Factors}

The following metric conversion factors are provided for standard units of measure used throughout this report:

$\begin{aligned} 1 \mathrm{in} . & =25.4 \mathrm{~mm} \\ 1 \mathrm{lb} & =0.453 \mathrm{~kg} \\ 1 \mathrm{gal} & =3.78 \mathrm{~L} \\ 1 \mathrm{psi} & =6.89 \mathrm{kPa} \\ 1 \text { ton (refrigeration) } & =3.516 \mathrm{~kW} \\ 1 \mathrm{Btu} & =1.055 \mathrm{~kJ}\end{aligned}$




\section{Cooling Options}

\section{Status Quo}

At present, three electric motor-driven chillers in the Davis-Monthan AFB cooling plant meet the cooling requirements of the medical facility: a York centrifugal chiller nominally of 250-tons capacity, and two Dunham-Bush screw machines of nominal 75-tons capacity each. Performance characteristics of the York chiller were obtained for part load operation and for condenser water return temperatures coincident with load as determined from analyzing cooling logs provided by the base. An earlier analysis contained information as to the fullload performance of the screw machines. Part-load performance was estimated using Figure 14 of Chapter 42, 1994 ASHRAE Refrigeration Handbook.

\section{Two Hundred Fifty (250)-Ton Capacity Natural Gas Engine-Driven Chiller With Heat Recovery (Option \#1)}

This option did not involve electrical power generation, but offered a potentially economical approach for meeting the facility's cooling loads. This hypothesis was based on the fact that previous analyses indicated this technology should be economically viable at other sites on the base. Under this option, the existing 250-ton capacity York motor-driven centrifugal chiller would be replaced by a natural gas engine-driven chiller using HCFC-22. The engine would be capable of delivering jacket water at temperatures varying between 183 and $201{ }^{\circ} \mathrm{F}$, depending on load, for heat exchange to produce water for space and domestic hot water heating. Return jacket water temperature would be $180^{\circ} \mathrm{F}$. Chiller performance took into account part load efficiencies and the return condenser water temperatures coincident with load as determined from analyzing cooling logs provided by the base. 


\section{Power Generation With Sufficient Waste Heat To Operate a Water Chilling Unit}

\section{Option \#2a: One Hundred (100)-Ton Capacity Single-Effect Indirect-Fired Absorption Chiller}

Analysis of the hospital's cooling loads indicated a year-round base load of 100 tons. Under this option, that base load would be met by a single-effect absorption chiller. The heat input would be provided by 15 psig steam produced from heat as the byproduct of power generation from a Caterpillar G3512 (600 kW) engine-generator set. Although the intent was to match, as far as possible, the estimated amount of "waste" heat to the chiller heat input required, some residual waste heat will result, which will be used to satisfy part of the facility's thermal load. Chiller performance was based on the assumption that the chiller would continuously provide 100 tons of cooling, but under variable condenser water return temperatures (as determined from logs provided by the base). Under this option (and for all [four] options involving a new base-loaded 100-ton capacity absorption chiller), the existing 250-ton capacity centrifugal chiller would not be removed. Replacing the existing chiller with only a 100-ton capacity absorption chiller would leave the plant short of capacity. (The data indicates there are periods when the total load on the plant exceeds 250 tons.) For this option and the next, the analysis is based on the assumption that, beyond 100 tons, screw machines will operate until the plant load reaches approximately 175 tons, at which point the operating screw machine will shut off and the 250-ton capacity centrifugal chiller will come on. The new 100-ton capacity absorption chiller would be located in the vicinity of the enginegenerator set and heat recovery equipment.

\section{Option \#2b: One Hundred (100)-Ton Capacity Double-Effect Indirect- Fired Absorption Chiller}

As stated above, analysis of the hospital's cooling loads indicated a year-round base load of 100 tons. Under this option, that base load would be met by a double-effect absorption chiller; the engine-generator set is a Caterpillar G-3516 $(820 \mathrm{~kW})$. The double-effect absorption chiller is about 50 percent more efficient than the single-effect unit. However, the double-effect requires a steam input at higher temperature - at the temperature of 115 psig versus the 15 psig of the single-effect unit. Also, the first cost of the double-effect unit is higher than that of the single-effect chiller. The performance at reduced condenser water temperatures was assumed to be the same as that of the single-effect unit. Under this option, the existing 250-ton capacity centrifugal chiller would not be removed. Replacing the existing chiller with only a 100-ton capacity absorption chiller 
would leave the plant short of capacity. (The data indicates there are periods when the total load on the plant exceeds 250 tons.) The analysis is based on the assumption that, beyond 100 tons, screw machines will operate until the plant load reaches approximately 175 tons, at which point the operating screw machine will shut off and the 250-ton capacity centrifugal chiller will come on. The new 100-ton capacity absorption chiller would be located in the vicinity of the engine-generator set and heat recovery equipment.

\section{Option \#2c: Two Hundred Fifty (250)-Ton Capacity Single-Effect Indirect- Fired Absorption Chiller}

Under this option, two $820 \mathrm{~kW}$ Caterpillar G-3516s would be selected so that the waste heat matches the heat input requirement for the chiller as closely as possible. All residual heat over what the chiller requires will be used to at least partially meet the facility's thermal requirements (also like the two preceding options). However, the new indirect-fired absorption chiller under this option would be able to produce 250 tons of cooling, matching the capacity of the existing centrifugal chiller. Under this option (and for all [four] options involving a new 250-ton capacity absorption chiller), the existing centrifugal chiller will be physically replaced by the new absorption unit. Chiller performance characteristics were based not only on return condenser water temperature, but also on load since the intent is for the absorption chiller to meet all cooling loads up to the initial 250 tons. Above that threshold, one or both of the existing screw machines would operate.

\section{Option \#2d: Two Hundred Fifty (250)-Ton Capacity Double-Effect Indirect-Fired Absorption Chiller}

For this option, the engine-generator set (three $820 \mathrm{~kW}$ Caterpillar G-3516s) would be selected so that its waste heat matches the heat input requirement for the chiller as closely as possible. Any residual heat over what the chiller requires will be used to at least partially meet the facility's thermal requirements. However, the new double-effect indirect-fired absorption chiller under this option would be able to produce 250 tons of cooling, matching the capacity of the existing centrifugal chiller. Under this option, the existing centrifugal chiller will be physically replaced by the new double-effect indirect-fired absorption unit. Chiller performance characteristics were based not only on return condenser water temperature, but also on load since the intent is for the absorption chiller to meet all cooling loads up to the initial 250 tons. Above that threshold, one or both of the existing screw machines would operate. This option is similar in all respects to that immediately preceding it except that a more 
efficient double-effect absorption chiller would be installed, requiring 115 psig steam instead of 15 psig.

\section{Power Generation With Sufficient Waste Heat To Operate a Water Chilling Unit and Satisfy Facility Thermal Requirements}

\section{Option \#3a}

This option is identical to Option \#2a, except the engine-generator capacity is sized to meet the thermal requirements of the facility in addition to the energy requirements of the chiller. Under this option, that base load would be met by a single-effect indirect-fired absorption chiller. The heat input would be provided by 15 psig steam produced from heat as the byproduct of power generation from two Caterpillar G3516 ( $820 \mathrm{~kW}$ ) engine-generator sets. The intent was to match the estimated amount of "waste" heat to the chiller heat input required and satisfy the facility's thermal load. Chiller performance was based on the assumption that the chiller would continuously provide 100 tons of cooling, but under variable condenser water return temperatures (as determined from logs provided by the base). Under this option, the existing 250-ton capacity centrifugal chiller would not be removed. Replacing the existing chiller with only a 100-ton capacity absorption chiller would leave the plant short of capacity. For this option, the analysis is based on the assumption that, beyond 100 tons, screw machines will operate until the plant load reaches approximately 175 tons, at which point the operating screw machine will shut off and the 250-ton capacity centrifugal chiller will come on. The new 100-ton capacity single-effect indirect-fired absorption chiller would be located in the vicinity of the enginegenerator set and heat recovery equipment.

\section{Option \#3b}

This option is identical to Option $\# 2 b$, except the engine-generator capacity is sized to meet the thermal requirements of the facility in addition to the energy requirements of the chiller. Analysis of the hospital's cooling loads indicated a year-round base load of 100 tons. Under this option, that base load would be met by a 100 ton, double-effect, indirect-fired absorption chiller; the engine-generator sets are four Caterpillar G-3516s (820 kW). The double-effect absorption chiller is about 50 percent more efficient than the single-effect unit. However, the double-effect requires a steam input at higher temperature - at the temperature of 115 psig versus the $15 \mathrm{psig}$ of the single-effect unit. Also, the first cost of the double-effect unit is higher than that of the single-effect chiller. The performance at reduced condenser water temperatures was assumed to be the same as 
that of the single-effect unit. Under this option, the existing 250-ton capacity centrifugal chiller would not be removed. Replacing the existing chiller with only a 100-ton capacity absorption chiller would leave the plant short of capacity. The analysis is based on the assumption that, beyond 100 tons, screw machines will operate until the plant load reaches approximately 175 tons, at which point the operating screw machine will shut off and the 250-ton capacity centrifugal chiller will come on. The new 100-ton capacity absorption chiller would be located in the vicinity of the engine-generator set and heat recovery equipment.

\section{Option \#3c}

This option is identical to Option $\# 2$ c, except the engine-generator capacity is sized to meet the thermal requirements of the facility in addition to the energy requirements of the chiller. Under this option, three $820 \mathrm{~kW}$ Caterpillar G-3516s would be selected so that the waste heat matches the heat input requirement for the chiller and will meet the thermal requirements of the facility. The new single-effect indirect-fired absorption chiller under this option would be able to produce 250 tons of cooling, matching the capacity of the existing centrifugal chiller. Under this option, the existing centrifugal chiller will be physically replaced by the new absorption unit. Chiller performance characteristics were based not only on return condenser water temperature, but also on load since the intent is for the absorption chiller to meet all cooling loads up to the initial 250 tons. Above that threshold, one or both of the existing screw machines would operate.

\section{Option \#3d}

This option is identical to Option \#2d, except the engine-generator capacity is sized to meet the thermal requirements of the facility in addition to the energy requirements of the chiller. For this option, the engine-generator set, six $820 \mathrm{~kW}$ Caterpillar G-3516s, would be selected so that its waste heat matches the heat input requirement for the chiller and meets the thermal requirements of the facility. However, the new double-effect indirect-fired absorption chiller under this option would be able to produce 250 tons of cooling, matching the capacity of the existing centrifugal chiller. The existing centrifugal chiller will be physically replaced by the new double-effect indirect-fired absorption unit. Chiller performance characteristics were based not only on return condenser water temperature, but also on load since the intent is for the absorption chiller to meet all cooling loads up to the initial 250 tons. Above that threshold, one or both of the existing screw machines would operate. This option is similar in all respects to that immediately preceding it except a more efficient double-effect absorption chiller would be installed, requiring 115 psig steam instead of 15 psig. 


\section{Boiler Load and Heating Requirements}

The maximum hourly boiler load was found from the daily boiler logs to be 3,491 pounds per hour (\#/hr) of steam at 0800 on 1 February 94 . With the steam produced at $50 \mathrm{psig}$, this equates approximately to 3,491 \#/hr x $912 \mathrm{Btu} / \#=3,184$ $\mathrm{MBH}$. The steam was used indirectly for space heating and domestic hot water production, and directly for humidification, dining hall requirements, and medical equipment sterilization.

\section{Space Heating Requirements}

Based on the time of day and year, the space heating requirement was probably very close to that of the basis of design. Schedules on the design drawings were used to calculate design space heating loads, as follows: $(13,400+4,570) \mathrm{gal} / \mathrm{hr} \mathrm{x}$ $\mathrm{hr} / 60 \mathrm{~min} \times 500 \times(150-130)^{\circ} \mathrm{F}=2,995 \mathrm{MBH}$.

\section{Direct Steam Requirements}

Previous analysis estimated that direct steam usage constitutes some 5 percent of the boiler output. On that basis, the direct steam used was about $0.05 \times 3,184$ $\mathrm{MBH}=159 \mathrm{MBH}$. Requirements for direct steam usage will be substantially reduced as the dining portion of the hospital will be eliminated. Further, local sterilizers are now being used in some instances instead of imported steam from the central heat plant. Clearly, local humidifiers are also readily available that can be used for humidification, without use of imported steam.

\section{Domestic Hot Water Heating Requirements}

The remainder of the boiler output is attributed to domestic hot water production, or: 
This figure is in line with an estimate found in an earlier analysis, but is small compared to the domestic hot water heating capacity determined from the schedule on the design drawings, which is:

$640 \mathrm{gal} / \mathrm{hr} \times 62.4 \mathrm{\#} / \mathrm{cu} \mathrm{ft} / 7.48 \mathrm{gal} / \mathrm{cu} \mathrm{ft} \times 1 \mathrm{Btu} / \#{ }^{\circ} \mathrm{F} \times 80^{\circ} \mathrm{F}=427 \mathrm{MBH}$

It may well be that the domestic hot water load was grossly overestimated, similar to the required boiler capacity, or there may have actually been, at one time, that much load. Mr. Domako of the Base Civil Engineering (BCE) staff has indicated that he anticipates domestic hot water load will decrease in the future as the hospital is converted to an outpatient facility.

The maximum thermal load for the purposes of this analysis will be considered to be the sum of the space heating and domestic hot water load, above, or 3,025 $\mathrm{MBH}$. The direct steam requirements will not be provided by way of waste heat. Those requirements should be met by continuing the trend toward local sterilization and installation of grid (or other type) humidifiers at the air handling units. This will permit the plant to be unmanned. The thermal space and domestic hot water heating loads will still be met as they are already satisfied by 15 psig steam from a pressure reducing valve station from the central heat plant boilers. The thermal energy requirements will be determined from the monthly boiler logs for Summer and for Winter (Table 1), and will be compared against the Btus of waste heat energy generated for those seasons to see if there will be an overall surplus or shortfall of energy.

Table 1. Identification of energy requirements from monthly boiler logs for 1995.

\begin{tabular}{|c|c|}
\hline Summer (May - Oct): & Energy Requirements (\# of Steam) \\
\hline May & $1,551,000$ \\
\hline June & $1,163,100$ \\
\hline July & 902,500 \\
\hline August & 703,600 \\
\hline September & 841,800 \\
\hline October & $1,200,800$ \\
\hline Summer Total $=$ & $6,362,800(\times 912$ Btu/\# $=5,802.874 \mathrm{Mbtu})$ \\
\hline Winter (Nov - Apr): & \\
\hline November & $1,200,800$ \\
\hline December & $1,559,800$ \\
\hline January & $1,622,700$ \\
\hline February & $1,334,200$ \\
\hline March & $1,369,200$ \\
\hline April & $1,766,200$ \\
\hline Winter Total = & $8,852,900=(x$ 912 Btu/\# = 8,073.845 Mbtu) \\
\hline * Indicates data that was averaged using September and December data \\
\hline
\end{tabular}




\section{Heat Recovery}

Heat recovery is a feature of all options considered except (of course) the base option.

\section{Option \#1}

Under this option, heat is potentially recoverable from both the engine and the exhaust. Due to the fact there would be light load conditions experienced during the year, USACERL was advised that heat should be extracted from the engine only. Extracting heat at low load will lower the temperature of the exhaust gas to the point where some condensation will occur with resulting corrosion. Consequently, only heat recovery from the engine was considered.

\section{Remaining Options (Heat Recovery from Engine-Generator Sets)}

Remaining options considered heat recovery from the engine and/or the exhaust, depending on whether high (115 psig) or low (15 psig) pressure steam was required (i.e., depending on whether a double or single-effect absorption chiller was under consideration). Low load that would promote corrosion is not a problem - the engine-generator sets, when operating, would be running at full load, generating the maximum amount of electricity possible. As pointed out earlier in the discussion regarding options, the amount of heat recovery considered was either that necessary to operate the absorption chiller at full load, with any excess used to offset facility thermal requirements, or was that required to operate the absorption chiller at full load and to meet the facility thermal load as well. The analysis does not allow more heat energy to be recovered than required. This limitation is imposed seasonally (Winter, Summer) and thus is somewhat broad. For cases where equipment is sized to produce waste heat to operate the chiller and meet the facility thermal load, the seasonal excess of energy available over required is sufficiently large that short duration thermal requirements should still be met for the vast majority of the time. The excess heat would be "dumped" to the generously sized existing cooling tower that currently cools the condenser water for the existing 250-ton capacity motor-driven centrifugal chiller. USACERL acknowledges and appreci- 
ates the assistance of Mr. Warner Bauer of Engineering Controls, Inc., St. Louis, $\mathrm{MO}$, in selecting the engine-generator models and quantities, and heat recovery equipment that would meet the performance criteria under each option considered. Capital costs for the equipment were also provided by Engineering Controls. The equipment selections and capital costs as provided by Engineering Controls, Inc. appear in Appendix B. Note that Waukesha and Caterpillar selections were made so that sole source procurement would not be required and to ensure that performance should be comparable. 


\section{Utility Rate Structures}

\section{Electrical Rates}

Appendix C contains recent monthly electric bills for Davis-Monthan AFB for a year (with the exception of September, for which interpolated data was used). Using the electric bills, the rates were determined for use in the spreadsheet. The basic demand charge of $\$ 10.28 / \mathrm{bill}$ able $\mathrm{kW}(\mathrm{bkW})$ is applicable for the entire year. The demand charge is apparently subject to 5 percent Arizona state sales tax, applied to 92 percent of the total (demand plus energy charges) due to a hospital exemption. Power factor adjustment and the Arizona Corporation Commission Assessment were not considered as individually they are well within the "noise" level of the total monthly electric charge and their difference (the former is typically a credit, the latter a debit) is even more so. Consequently, the basic demand cost was figured as:

$$
\$ 10.28 / \mathrm{bkW} \times \mathrm{bkW}=\text { demand cost }(\mathrm{DC})
$$

upon which the sales tax is levied, subject to the allowable exemption, or:

$$
\mathrm{DC}=\$ 10.28 \times \mathrm{bkW}+\$ 10.28 \times \mathrm{bkW} \times 0.92 \times 0.05
$$

so that the actual demand cost would be:

$$
D C=\$ 10.28 \times b k W \times(1+0.92 \times 0.05)=\$ 10.75 \times b k W .
$$

The electrical energy rate is subject to the same levy. The basic Summer rate is $\$ 0.047457 / \mathrm{kWh}$ and the basic Winter rate is $\$ 0.045084 / \mathrm{kWh}$. Adjusted for the same levy as applied to the demand charge, the rates become, respectively:

$$
1.046 \times \$ 0.047457 / \mathrm{kWh}=\$ 0.0496 / \mathrm{kWh}
$$

and

$$
1.046 \times \$ 0.045084 / \mathrm{kWh}=\$ 0.0472 / \mathrm{kWh} .
$$


Additionally, the electrical rate schedule includes a 0.667 ratchet applied to the peak KW demand experienced over the previous 11 months. The contract with Tucson Electric Power Company includes a minimum monthly buy of 3,000 kW. None of the options regarding power generation will penetrate this floor, based on the data in Appendix C.

\section{Natural Gas Rates}

Information received from $\mathrm{Mr}$. Weleck of the BCE staff indicated the gas rates are now $\$ 2.75 / \mathrm{MBtu}$ in Summer and $\$ 3.90$ in Winter. 


\section{Methodology for Analysis}

Based on the cooling load profile, hours at various loads were determined. The spreadsheet then modeled how these loads would be met, either using the existing cooling equipment or the equipment described above under the various options considered. Note that the hours at the various loads do not total the entire hours of the year $(8,760)$, although there is apparently a requirement for year-round cooling. This simplification was introduced since, once a load over 250 tons is experienced, the equipment that will operate to meet that additional load will be the same under any of the options considered. However, for options involving onsite electrical power generation, all hours of the year were considered, except for the estimated periods of time when the equipment would be inoperative for maintenance (95 and 98 percent availability on average in Summer and Winter, respectively). The hours of operation are divided between Summer (May through October, indusive) and Winter (November through April) due to the variation in utility rates between the two seasons. The enginegenerator sets have been derated to account for altitude (Tucson's elevation is 2,654 feet above sea level) and outdoor temperature.

Maintenance costs for the Caterpillar G3516 were based on a previous analysis by Empire Power Systems, Phoenix, AZ. This included all parts and labor for oil changes, makeup oil, scheduled preventive maintenance, overhauls, unscheduled stoppages due to out-of-tolerance conditions, etc. The maintenance required for the G3512 was assumed to be essentially equal to that of the G3516. An interruption for over 15 minutes in the monthly operation of a given generator will render demand savings from that generator moot for the month (although savings in billable demand may still be possible where operation has reduced peak and the month is one in which billable demand would exceed actual demand). This would be the case regardless of the estimated overall Summer 95 percent or Winter 98 percent availability rates. If units must be down, clearly the preference is to take them down during periods of months when demand is relatively low, keeping the units in service during months when peak demands are typically experienced. Preference should be given to pulling operationally interruptive maintenance during months when billable demand typically exceeds actual demand. It is, of course, recognized that this isn't always possible. The operational assumptions made in the spreadsheet are based on operating the equipment according to the recommendations above, but allowing for the 
possibility that unscheduled events will occur. Operating scenarios were developed for all options based on the practices recommended above and are indicated in the following.

\section{Option \#2a}

With only one Caterpillar G3512 engine-generator set, it was assumed that there would be totally uninterrupted service for 3 of the 6 summer months. It is assumed that, of the 3 months, due diligence has been taken to ensure uninterrupted operation for 2 of the 3 months with the highest actual demand, but the unit went down for the month with the third-highest demand. For Winter, it is assumed that there will be uninterrupted service for 3 months, and interrupted service for 3 months. (Actually, it really does not matter from a demand reduction viewpoint whether the engine-generator does or does not operate during months in which the minimum billable amount is determined by the ratchet; i.e., November to February, inclusive.) Appendix D indicates the situation, incorporating the data from Appendix E. Table 2 lists the projected demand reductions by month.

For input to the spreadsheet, there would be 3 months in Summer when the demand reduction would be $555 \mathrm{~kW}$, and for Winter, there would be 4 months for which the reduction would be $370 \mathrm{~kW}$ and 1 month for which the reduction would be $555 \mathrm{~kW}$.

\section{Option \#2b}

This option is considered identical to Option \#2a from an operational standpoint. However, the power production is greater for the Caterpillar G3516 engine-generator that would be installed under this option. Appendix $E$ shows the results. Note again that, in Winter, for the 4 months when the ratchet is in effect, it does not matter from a demand reduction perspective whether any unit does or does not operate. This pattern is characteristic for all the options involving onsite power production.
Table 2. Projected demand reductions, by month.

\begin{tabular}{|l|c|}
\hline Jul & $555 \mathrm{~kW}$ \\
\hline Aug & $555 \mathrm{~kW}$ \\
\hline Sep & $0 \mathrm{~kW}$ \\
\hline Oct & $555 \mathrm{~kW}$ \\
\hline Nov & $370 \mathrm{~kW}(=12,543-12,173)$ \\
\hline Dec & $370 \mathrm{~kW}$ \\
\hline Jan & $370 \mathrm{~kW}$ \\
\hline Feb & $370 \mathrm{~kW}$ \\
\hline Mar & $0 \mathrm{~kW}$ \\
\hline Apr & $555 \mathrm{~kW}$ \\
\hline May & $0 \mathrm{~kW}$ \\
\hline Jun & $0 \mathrm{~kW}$ \\
\hline
\end{tabular}




\section{Option \#2c (also applicable to Option \#3a)}

Under this option, it is assumed that in Summer, one Caterpillar G3516 unit will be available to achieve demand reductions for 3 months and two such units will be available to achieve reductions for the remaining 3 months. It is assumed that Winter operation will replicate Summer. Appendix F shows operation for the year. Since two Caterpillar G3516 engine-generators are being considered for Option \#3a, the same results apply for that option.

\section{Option \#2d (also applicable to Option \#3c)}

This option involves three Caterpillar G3516 engine-generator sets. It is assumed that in Summer, all three will be available for 2 months and two will be continuously available for 4 months. In Winter, the same assumption is made. Appendix $G$ shows the results. The same results also apply to Option \#3c.

\section{Option \#3b}

This option involves four Caterpillar G3516 engine-generator sets. It is assumed that in Summer all four will be available for 2 months and three will be available for the remaining 4 months. In Winter, the same availability is assumed. Appendix $\mathrm{H}$ shows the results.

\section{Option \#3d}

Six Caterpillar G3516 engine-generator sets were considered for installation under this option. For operation in Summer, it is assumed that all six units will operate for 2 months, five units will operate continuously for 2 months, and four units will be maintained in continuous operation for 2 months. Winter operation will be assumed to be identical. Appendix I shows the results.

\section{First Costs}

Appendix J contains the construction cost estimates for each option. The cost estimates for the heat recovery equipment and associated engine-generator sets 
were those provided by Engineering Controls, Inc., as previously discussed. All other costs were based on Means Mechanical Cost Data 1997.

The cost estimates do not include the construction of an enclosure for equipment located exterior to the central energy plant building. Estimating the cost of such a structure would be difficult since the architectural requirements vary for each base. However, it does include the cost of a concrete pad on which to install the equipment.

\section{Life Cycle Cost Analysis}

The life cycle cost analysis for each option was calculated using Life Cycle Cost In Design (LCCID) software.* The life cyde cost analysis accounts for the construction, overhead, and design costs associated with each option. The sum of these values is the total investment. The economic life is taken over a 20-year period and accounts for all scheduled maintenance activities as detailed by the manufacturer. No service contracts were considered as part of this study.

\footnotetext{
* Linda K. Lawrie, Technical Report (TR) E-85/07/ADA162522, Development and Use of the Life Cycle Cost in Design Computer Program (LCCID) (U.S. Army Construction Engineering Research Laboratories [USACERL], November 1985).
} 


\section{Results}

Appendix $\mathrm{K}$ shows the EXCEL ${ }^{\oplus}$ spreadsheet that contains the raw energy data. Appendixes $L$ to $T$ and Table 3 contain calculated payback and savings-toinvestment ratio for each option. The table below summarizes the economic results. These results form the basis for the recommendations in the next paragraph. This report does not include potential savings that may be achievable under Options \#3a, 3b, 3c, and 3d by having an unmanned plant. Under these options, there would be initial first cost incurred due to installation of humidification units at the air handling units and use of sterilizers where the energy input for sterilization would be at the point of use. Estimating overall savings through removing the plant manning requirement was beyond the scope of a cooling study. However, in addition to the considerable utility savings identified for Options \#3a through 3d, inclusive, there would likely be considerable savings from eliminating the requirement for a manned plant.

Table 3. Calculated payback and savings-to-investment ratios for options.

\begin{tabular}{|c|c|c|c|c|}
\hline Option & Payback (years) & SIR & Total Investment & Recommendation \\
\hline $3 a$ & 1.01 & 9.29 & $\$ 269,507$ & implement \\
\hline $3 b$ & 1.21 & 6.61 & $\$ 536,817$ & implement \\
\hline $3 c$ & 1.49 & 5.83 & $\$ 538,392$ & \\
\hline $3 d$ & 1.43 & 5.44 & $\$ 934,974$ & \\
\hline $2 c$ & 1.65 & 5.96 & $\$ 476,529$ & \\
\hline $2 d$ & 1.75 & 4.96 & $\$ 639,087$ & \\
\hline $2 b$ & 2.51 & 2.77 & $\$ 235,412$ & \\
\hline $2 a$ & 2.87 & 2.32 & $\$ 196,252$ & \\
\hline 1 & 9.35 & 1.32 & $\$ 290,974$ & do not implement \\
\hline
\end{tabular}




\section{Conclusions and Recommendations}

The medical facility at Davis-Monthan AFB can realize considerable utility cost savings by implementing any of several options analyzed in this study. It is likely such savings can be replicated at other DOD medical facilities where there are year-round air conditioning requirements, large thermal energy requirements, and utility rates where the unit cost of purchased electricity is high compared to that of natural gas.

Based on these results, this study recommends the projects be prioritized for implementation, from most to least highly recommended, as follows:

1. Option 3a: Two natural gas-fired Caterpillar G-3516 (820 kW) enginegenerator sets, waste heat used to provide steam for a 100 ton single-effect indirect-fired absorption water chiller to meet facility base cooling load (100 tons) with residual heat adequate to satisfy facility thermal requirements (existing boilers as backup only). This option is recommended for implementation.

2. Option 3b: Four natural gas-fired Caterpillar G-3516 (820 kW) enginegenerator sets, waste heat used to provide steam for a 100 ton double-effect indirect-fired absorption water chiller to meet facility base cooling load (100 tons) with residual heat adequate to satisfy facility thermal requirements (existing boilers as backup only). This option is recommended for implementation.

3. Option 3c: Three natural gas-fired Caterpillar G-3516 (820 kW) enginegenerator sets, waste heat used to provide steam for 250 ton single-effect indirect-fired absorption water chiller to replace existing 250-ton motordriven centrifugal chiller, with residual heat adequate to satisfy facility thermal requirements (existing boilers as backup only).

4. Option 3d: Six natural gas-fired Caterpillar G-3516 (820 kW) enginegenerator sets, waste heat used to provide steam for a 250 ton double-effect indirect-fired absorption water chiller to replace existing 250-ton motordriven centrifugal chiller, with residual heat adequate to satisfy facility thermal requirements (existing boilers as backup only). 
5. Option 2c: Two natural gas-fired Caterpillar G-3516 (820 kW) enginegenerator sets, waste heat used to provide steam for a 250 ton single-effect indirect-fired absorption water chiller to replace existing 250-ton motordriven centrifugal chiller, with residual heat used for thermal requirements.

6. Option 2d: Three natural gas-fired Caterpillar G-3516 (820 kW) enginegenerator sets, waste heat used to provide steam for a 250 ton double-effect indirect-fired absorption water chiller to replace existing 250-ton motordriven centrifugal chiller, with residual heat used for thermal requirements.

7. Option 2b: One natural gas-fired Caterpillar G-3516 (820 kW) enginegenerator set, waste heat used to provide steam for a 100 ton double-effect indirect-fired absorption water chiller to meet facility base cooling load (100 tons) with residual heat used for thermal requirements.

8. Option 2a: One natural gas-fired Caterpillar G-3512 (600 kW) enginegenerator set, waste heat used to provide steam for a 100 ton single-effect indirect-fired absorption water chiller to meet facility base cooling load (100 tons) with residual heat used for thermal requirement.

9. Option 1: A 250 ton natural gas engine-driven chiller to replace existing 250ton motor-driven centrifugal chiller, with waste heat used to offset facility thermal requirements. Note that this option is not recommended for implementation. 


\section{Appendix A: Notes Regarding Interviews and Discussions for Chiller Study at Davis-Monthan AFB}

SSgt Rinn, TSgt Farmer, Central Plant Operators -

A. As of 1 Apr 97, the foregoing operators have been on temporary assignment pending award of a contract for private sector O\&M for the hospital, including the central plant. The operators provided chilled water logs for the previous year (to be returned to Steve Weleck). They also furnished monthly boiler logs for the months ( $\mathrm{J}$ an - J un 97, logs to be returned to Weleck) and referred us to Lt. Doolittle for the balance of the previous year's monthly boiler logs. Lt. Doolittle furnished CERL with monthly logs from J an 94 - Sep 95. In an attempt to determine peak plant thermal load, CERL went back to boiler log data for Feb 94 (records to be returned to Weleck).

B. Upon initial arrival, CERL was appraised that a cogeneration study had already been completed, Dec 95, with design drawings based on the study results also provided. The study and drawings were loaned to CERL for review, and CERL will return same to Weleck. While meeting with Weleck, CERL learned that two significant projects are planned for the hospital, a 6,000 SF addition and a follow-on 30,000 SF addition (both projects discussed in greater detail below). Additionally, Weleck furnished a document "FY88 MCP ECIP Facility Energy Improvements" for CERL's perusal to see if it might contain helpful information. Mr. Weleck also provided utility rate schedules and sample billings.

C. SSgt Rinn and TSgt Farmer retrieved plant drawings on file for CERL's review, particularly drawings containing schedules for space heating and domestic hot water equipment. Inquiry verified there is no separate metering of steam usage - some is used for space heating, some for domestic hot water production, and the remainder is direct steam usage. Therefore, the schedule sheets were used with other 
input to determine the relative uses for the steam produced. Of prime concern was the amount of steam used directly for various applications (dining facility, humidification, and medical sterilizers). The requirement for high pressure steam ( $>15$ psig [the plant operates at $50 \mathrm{psig]}$ ) drives the requirement for 24-hour manning of the plant.

D. Lt. Chicotah, facility manager at the hospital, was contacted by phone in an attempt to find out about future projects planned for the hospital. She Faxed a Preliminary Statement of Work for design of a FY00 30,000 SF addition (Ambulatory Health Care Center) to the existing medical facility. This is in addition to the $6,000 \mathrm{SF}$ Aerospace Medicine Clinic programmed for FY98. Neither this document nor the DD F orm 1391 or the Requirements and Management Plan (the latter two provided by SMSgt Mortenson) provided insight as to the mechanical equipment that is anticipated for use in heating and cooling either the planned 6,000 SF addition or the 30,000 SF addition. In a final attempt to get this information, CERL called Horace Hopper of AFCEE. The information Hopper currently has basically leaves the issue of the mechanical equipment to be used for the facility additions at the discretion of the designer. At this time, per Mr. Hopper, the designer has not been selected (contrary to information provided by Lt. Chicotah who indicated an A-E contract is to be awarded by the end of J uly 97). Numerous references were made to a Capt Reinhardt in San Francisco as the prime source of information regarding future plans for the hospital. Attempts to reach Reinhardt to date have been unsuccessful; however, CERL's plan is to use his information to resolve any conflicting or missing information. Mr. Ken Domako indicated he would attempt to resolve the issue of future requirements for direct steam use. He also indicated his estimate that domestic hot water usage at this time has been reduced significantly from what was the original basis of design, and that future requirements will likely be only about half that of the original basis of design. This is due to removal of the dining facility and elimination of showers for patients. Mr. Domako stated that the two planned hospital projects will be undertaken. 


\section{Approach}

A. Contact Capt Reinhardt regarding future hospital requirements.

B. Compute heating loads (process steam, space heating, and domestic hot water).

C. Plot cooling load profiles.

D. Consider the following options:

1. gas fired dual-effect absorption unit

2. gas engine-driven chiller

3. cogen system made up of a generator and indirect fired absorption unit

E. Analyze data and produce report.

\section{Determination of Heating Loads}

A. The existing boilers in the hospital central plant are greatly oversized. They are products of Nebraska Boiler, each rated at 7.5 million British Thermal Units per hour ( $\mathrm{MBH}$ ) with a mass flow of 7,000 pounds per hour (\#/hr) of 50 pounds per square inch gauge (psig) saturated steam. Examination of the boiler logs available indicated that the maximum boiler load experienced over the last 5 years (on 4 Feb 94) was 3,500 \#/hr, which translates into a heating load of

\section{3,500 \#hr x 7,500,000 Btu/7,000 \#/hr =3,750,000 Btu/hr}

This indicates a peak load of only 25 percent of the existing plant boiler capacity $(3.75 \mathrm{MBH} /[2 \times 7.5 \mathrm{MBH}])$. Most of the time, the load is significantly less. The load will decrease even more due to the factors described in the following paragraphs.

B. Domestic Hot Water Loads

Mr. Domako indicated that hospital care is going to be limited basically to outpatient care, including outpatient surgery. Showers for patients will be significantly reduced compared to that anticipated when the plant was constructed. Mr. Domako's estimate of the reduction is 50 percent. There are currently two domestic hot water generators in the plant, the original design intent being that either 
one could provide 100 percent redundancy. The schedule on the design drawings indicates the generators are capable of heating 640 gal of water from 60 to $120^{\circ} \mathrm{F}$ in 1 hour. The design also provided for what appears to be a booster heater to heat the water initially from 60 to $90^{\circ} \mathrm{F}$, with the $90^{\circ} \mathrm{F}$ water then heated to $120^{\circ} \mathrm{F}$ by the hot water generators. Since the heating from the booster actually supplants (although it also accelerates) the heating that would otherwise be required by the domestic hot water generators (increasing the domestic hot water temperature from 60 to $90{ }^{\circ} \mathrm{F}$ ), only the full capacity of the hot water generators need be calculated, which is

$$
\begin{gathered}
640 \mathrm{gal} / \text { hour } \times 8.34 \mathrm{lb} / \mathrm{gal} \times 1 \mathrm{Btu} / \mathrm{lb}{ }^{\circ} \mathrm{F} \times(120-60){ }^{\circ} \mathrm{F} \\
=320,256 \mathrm{Btu} / \mathrm{hr}
\end{gathered}
$$

Assuming a 50 percent reduction in load, the estimated domestic hot water heating requirement would be $320,256 \mathrm{Btu} / \mathrm{hr} / 2=160,128$ Btu/hr.

\section{Space Preheating, Heating, and Reheating Loads}

Drawings that contained schedules indicating the required capacities for preheat, heating, and reheat coils were reviewed. Rather than add these all up and then assume some diversity factor, the schedule for the converters was checked. Based on those schedules, the design heating water requirement was determined from

Converter \#1: $500 \times 13,400 \mathrm{gal} / \mathrm{hr} / 60 \mathrm{~min} / \mathrm{hr} \times(150-128)^{\circ} \mathrm{F}$

$$
=2,456,667 \mathrm{Btu} / \mathrm{hr}
$$

where operators indicated that the temperature of the supply hot water is $150^{\circ} \mathrm{F}$ and the return is typically the $128^{\circ} \mathrm{F}$ indicated.

Converter \#2: $500 \times 4,570 \mathrm{gal} / \mathrm{hr} / 60 \mathrm{~min} / \mathrm{hr} \times(150-128)^{\circ} \mathrm{F}$

$$
=837,333 \mathrm{Btu} / \mathrm{hr}
$$

Since both converters operate simultaneously, the capacities are summed to produce a joint capacity of $(2,456,667+837,333) \mathrm{Btu} / \mathrm{hr}=$ 3,294,500 Btu/hr. 
D. Process Steam L oads

Boiler plant steam is used directly for a number of purposes/ applications: sterilization, dining hall requirements, and humidification. Unfortunately, this steam (nor the domestic hot water or heating water used for space conditioning) has been metered. Therefore, the quantity of steam used directly (as opposed to heat exchange within the plant to produce domestic hot water and heating water for space conditioning) was calculated by subtracting the quantities in subparagraphs b) and c) from the peak steam load identified in subparagraph a). The quantity was calculated as:

3,750,000 Btu/hr - 160,171 Btu/hr - 3,294,500 Btu/hr =295,329 Btu/hr

Based on discussions with Mr. Domako and TSgt Mortenson, the dining hall will be eliminated and the space used for an alternative function. This will eliminate a portion of the present direct steam usage. Additionally, discussion with plant operators indicated that in a number of instances, steam from the plant is not being used for sterilization. Rather, portable units are being used for sterilization. Mr. Domako expects use of this type of sterilization to be expanded. He indicated he will check with medical personnel to try to ascertain future steam requirements. 


\title{
Appendix B: Equipment Selections and Capitol Costs
}

August 12, 1997

Budget $=2917$

Page 1 of 2

\author{
BUDGET COST FOR \\ U.S. ARMY CONSTRUCTION ENGINEERING \\ RESEARCH LABORATORIES
}

REF 2A: $1,714,300$ BTU/HR required at 15 PSIG.

Option *2a One Cateroillar G3512,600 KWe, 2,091,660 BTU/HR: \$54,560.00

One waukesha VHP-2900GSI, 425 KWe, 2,201,942 BTU/HR:

Feedwater unit adds:

$\$ 53,780.00$

$\$ 13,560.00$

REF $2 B: \quad 4,507,750$ BTU/HR required at 15 PSIG.

Option =2C Two Caterpillar G-3516, $820^{\circ}$ KWe, 2, 886,660

BTU/HR/Engine:

Two Haukesha VHP-2900GSI, 425 KWe, 2,201,942 BTU/HR/Engine:

Feecwater unit odds:

$\$ 108,480.00$

$\$ 101,240.00$

$\$ 19,340.00$

REF 2C: $1,000,000$ BTU/HR required at 115 PSIG.

Option \#2b

One Caterpillar G-3516, 820 Kwe, 1,010,000 BTU/HR:

One waukesha VHP-3600GSI, $550 \mathrm{KWe}, 1,063,000$ BTU/HR:

Feeculater unit adds:

$\$ 80,660.00$

$\$ 68,620.00$

$\$ 18,540.00$

REF 2D: 2,500,000 BTU/HR required at 115 PSIG.

Option $* 2 d$

Three Caterpillar G-3516, 820 Kwe, 1,010,000 BTU/HR/Engine:

Two waukesho $7100 \mathrm{GSI}, 1100 \mathrm{KWe}, 2,212,000$ BTU/HR/Engine:

Feedwater unit adds:

$\$ 232,920.00$

$\$ 183,500.00$

$\$ 23,920.00$

The 15 PSIG systems include the heat recovery unit, back pressure control valve, excess steam control valve and excess steam condenser.

The 115 PSIG systems include the heat recovery unit, back pressure control valve and external by-ocss tee. 
August 12, 1997

Budget \#2917

Poge 2 of 2

BUDGET COST FOR

U.S. ARNY CONSTRUCTION ENGINEERING RESEARCH LABORATORIES

REF 4A: 4,739,300 BTU/HR required at 15 PSIG. Option *3a

Two Cateroillar G3516, 820 KWe, 2,886,660 BTU/HR/Engine:

Two Woukesho VHP-7100GSI, $1100 \mathrm{KWe}, 5,487,129$ BTU/HR/Engine:

Feedwater unit odds:

$\$ 106,920.00$

$\$ 76,940.00$

$\$ 19,340.00$

REF 4B: $7,532,750$ BTU/HR required at-15 PSIG.

Option $=3 c$

Three Cateroillar G-3516, 820 KWe, 2, 886,660 BTU/HR/Engine:

Two Wqukesha VHP-5900GSI, 900 KWe, 4, 352, 552

BTU/HR/Engine:

Feedwater unit adds:

$\$ 154,080.00$

$\$ 100,100.00$

$\$ 22,840.00$

REF 4C: 4,025,000 BTU/HR required at 115 PSIG.

Option $\# 3 b$

Four Caterpiliar G-3516, 820 KWe, 1,010,000 BTL/HR:

Two Wqukesha VHP-7100GSI, 1100 KWe, 2,212,000

$\$ 312,620.00$ BTU/HR:

Feedwater unit adds:

$\$ 183,820.00$

$\$ 25,800.00$

REF 4D: 5,525,000 BTU/HR required at 115 PSIG.

\section{Option \#3d}

Six Caterpillar G-3516, 820 KWe, 1,010,000 BTU/HR/Engine:

Two Waukesho VHP-g500GSI, 1475 KWe, 3,058,312 BTU/HR/Engine:

Feedwater unit adds:

$\$ 461,220.00$

$\$ 224,660.00$

$\$ 30,460.00$

The 15 PSIG systems include the heat recovery unit, back pressure control valve, excess stean control valve and excess steam condenser.

The 115 PSIG systems include the heat recovery unit, back pressure control valve and external by-pass tee. 
13 Aug 97 phone discussion with Terry Hurley, Engineering Controls, St Louis, MO

Mr. Hurley provided the following namual gas flow rates for the Caterpillar engines, as follows:

$3512 \mathrm{TA} .600 \mathrm{KWe}$ - 100,431 Btw/minute 3516 TA $90,820 \mathrm{KWe} \longrightarrow 132,221$ Btu/minute

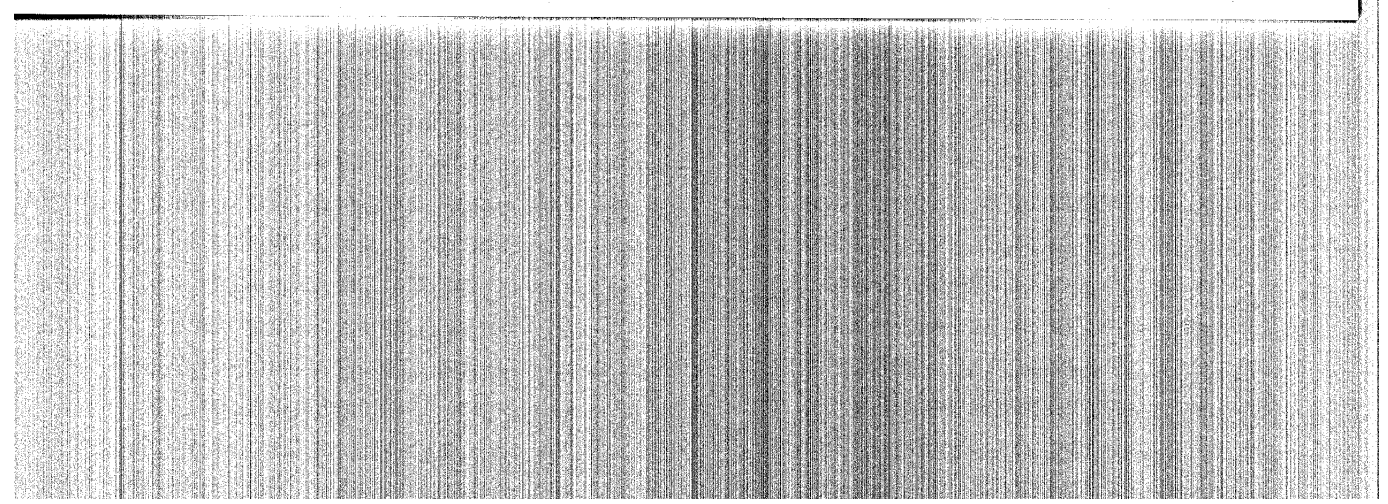




\section{Appendix C: Recent Electric Bills for Davis-Monthan AFB}


TUCSON ELECTRIC POWER COMPANY

P. O. 200711

Tueson, Arizona 85702

Dear Customer.

At your request, we submit cur Larģe Light anc Fower Rate No. 14 showing current adfusuments:

\section{LARGE LIGHT AND POWER RATE NO. 14}

\section{DEMAND CHARGE:}

Per $\mathrm{kW}$ of Billing Demand per month

ENERGY CHARGE:

All KWh par menth है $\begin{array}{ll}\text { Summer } & \text { Wintina Months } \\ \text { Nav-OC. } & \text { Nov-Aor. }\end{array}$

$\$ 10.28 \quad \$ 10.28$

BILLING DEMAND:

The Bining Demand shall be specified in the contract, but shall not be less than $3,000 \mathrm{~kW}$.

POWER FACTOR ACUUST:EYT

The above rate is stibiest to a dissunt or a tharga of $1.3 \mathrm{k}$ per $\mathrm{kW}$ of biling dernand for asch $1 \%$ the aversge monthly power factor is above or below $90 \%$ lagging to a maximum essiount of $13.0 \%$ per kW of biling demant per menth.

By law, the following tax and assessment gercenteges are applied where appropriate to calaulations on the above rates:

\section{FRANCHISETAX:}

(Tucson, South Tuessen)

CITY SALESTAX:

(Tucson, South Tueson, Marana)

\section{STATE SALES TAX}

(Applicable to all sales)

ARIZONA COFPORATION COMMISSION ASSFSSMENT: (Appicable to all saies)

RESIDENTIAL UTITTY CONSUMER ASSESSMENT:

(Residental Customers Onily)
$20 \%$

$20 \%$ (Tucson); $2.5 \%$ (South Tucsen); and 4.0\% (Marana) (aiso , applied to Avizena Corporation Comrission Assessment and Residental Utifly Consumer Assessment amounts)

$5.0 \%$ (aiso applied to City Franchise Tax amount and Avizona Corporaicn Commission Assessment and Residential Utility Consumer Assessment amounts)

$.14 \%$ (also applied to Cily Franchise Tax anount, City Sales Tax ancunt and State Sales Tax anount)

$.05 \%$ (also applied to City franchise Tax amount, Cily Saies Tex anount and State Sales Tax amount)

Very truly yours.

TUCSON ELECTRIC POWER COHAPANY

Effective August 1996 Bzlings

(Change due to revision of ACC and RUCO Assessments) 


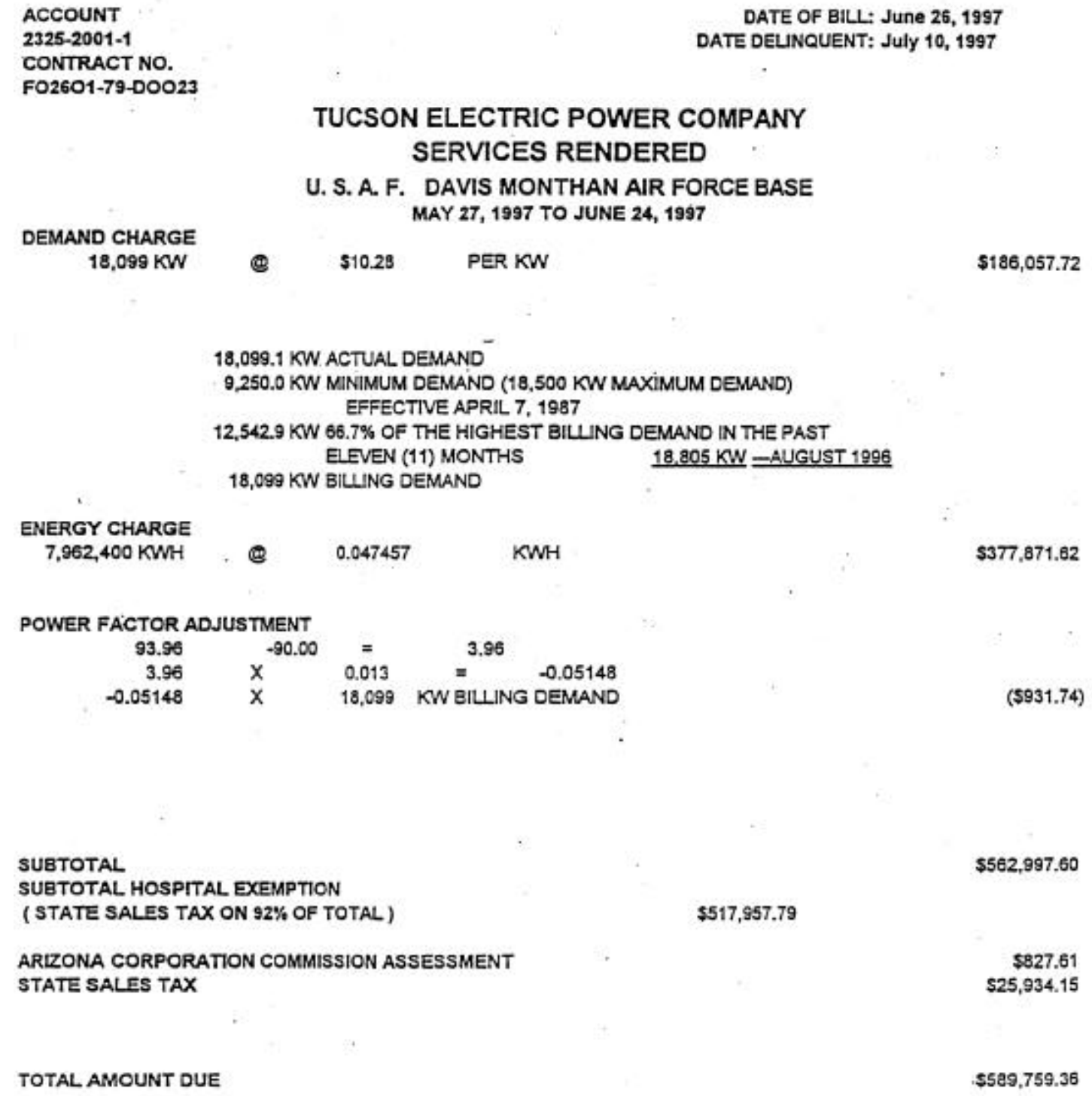 \\ U. S. A. F. DAVIS MONTHAN AIR FORCE BASE
MAY 27,1997 TO JUNE 24,1997 \\ U. S. A. F. DAVIS MONTHAN AIR FORC
MAY 27,1997 TO JUNE 24,1997 \\ TUCSON ELECTRIC POWER COMPANY}

DATE OF BILL: June 26, 1997 DATE DELINQUENT: July 10, 1997

$(\$ 931.74)$

$\$ 377,871.62$

SUBTOTAL

$\$ 562,997,60$

SUBTOTAL HOSPITAL EXEMPTION

(STATE SALES TAX ON $92 \%$ OF TOTAL)

$\$ 517, \$ 57.79$

ARIZONA CORPORATION COMMISSION ASSESSMENT

$\$ 827.61$

STATE SALES TAX

TOTAL AMOUNT DUE

$\$ 529,759.36$ 


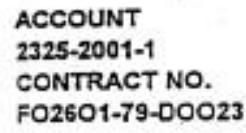

DATE OF BILL: May 29, 1997 DATE DELINQUENT: June 11, 1997

\section{TUCSON ELECTRIC POWER COMPANY SERVICES RENDERED}

DAVIS MONTHAN AIR FOR
APRIL 25, 1997 TO MAY 27, 1997

$-0.06279$

.

SUBTOTAL

$\$ 559,278,69$

ARIZONA CORPORATION COMMISSION ASSESSMENT

$\$ 822.14$

STATE SALES TAX

$\$ 28.003 .08$

CURRENT AMOUNT DUE

$\$ 588,103.91$

PREVIOUS BALANCE

$\$ 457,844.38$

TOTAL AMOUNT DUE

$\$ 1,045,948.29$ 
zCOUNT

125-2001-1

JNTRACT NO.

J2601-79-00023

EMAND CHARGE
$14,142 \mathrm{KW}$
DATE OF BILL: April 30, 1997 DATE DELINQUENT: May 13, 1997

\section{TUCSON ELECTRIC POWER COMPANY SERVICES RENDERED}

U.S. A. F. DAVIS MONTHAN AIR FORCE BASE MARCH 24, 1997 TO APRIL 25, 1997

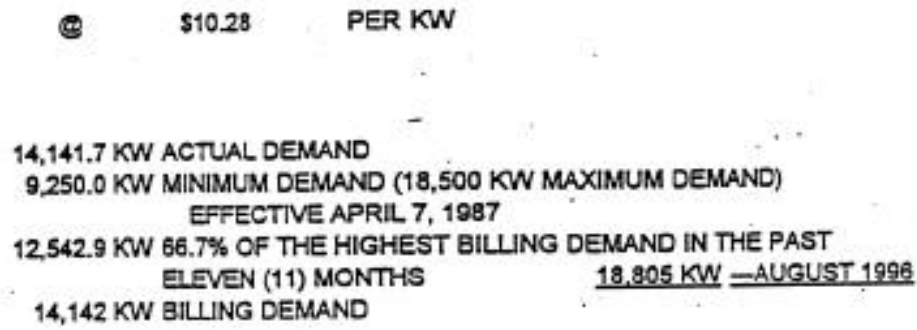

$\$ 145,379.76$

$(\$ 543.46)$

JBTOTAL

JTAL AMOUNT DUE 


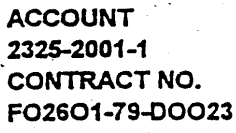

PER KW

$\$ 129,661.64$

\section{TUCSON ELECTRIC POWER COMPANY SERVICES RENDERED}

\section{U. S. A. F. DAVIS MONTHAN AIR FORCE BASE} FEBRUARY 25, 1997 TO MARCH 24, 1997

\section{DATE DELINQUENT: April 8, 1997}

DATE OF BILL: March 26, 1997

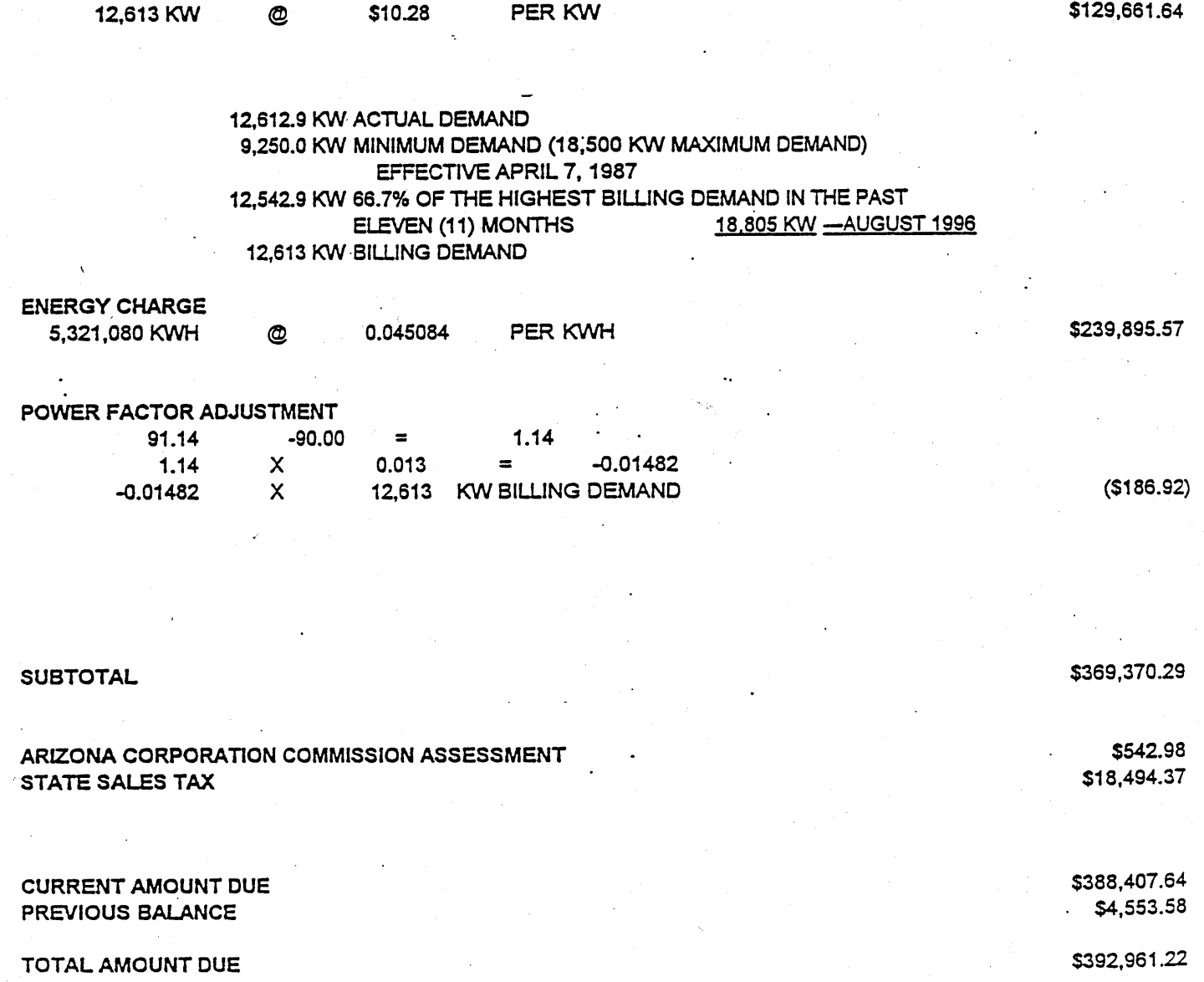




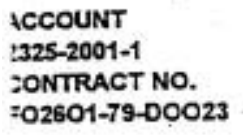

TUCSON ELECTRIC POWER COMPANY

U. S. A. F. DAVIS MONTHAN AIR FORCE BASE JANUARY 24, 1997 TO FEBRUARY 25, 1997

\section{SERVICES RENDERED}

SUBTOTAL 
ACCOUNT

2325-2001-1

CONTRACT NO.

FO2601-79-DOO23

DEMAND CHARGE

$12,543 \mathrm{KW}$

$\ldots$

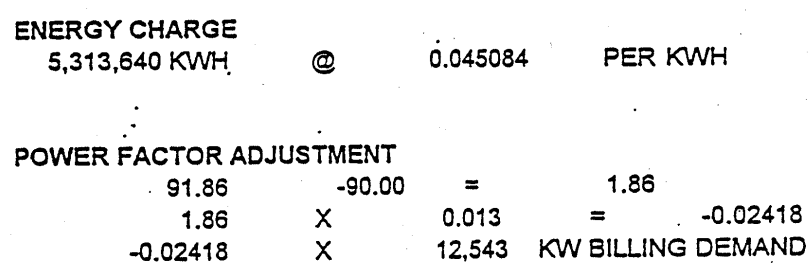

SUBTOTAL

$12,543 \quad K W$ BILLING DEMAND

\section{TUCSON ELECTRIC POWER COMPANY SERVICES RENDERED} DECEMBER 26, 1996 TO JANUARY 24, 1997

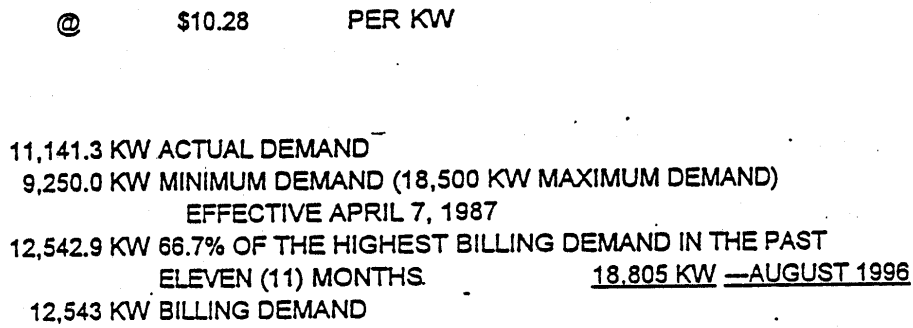

11,141.3 KW ACTUAL DEMAND ${ }^{-}$

9,250.0 KW MINIMUM DEMAND $(18,500 \mathrm{KW}$ MAXIMUM DEMAND)

EFFECTIVE APRIL 7, 1987

$12,542.9 \mathrm{KW} 66.7 \%$ OF THE HIGHEST BILLING DEMAND IN THE PAST

ELEVEN (11) MONTHS. $12,543 \mathrm{KW}$ BILLING DEMAND

DATE OF BILL: January 28, 1997 DATE DELINQUENT: February 10, 1997

$(\$ 303.29$

ARIZONA CORPORATION COMMISSION ASSESSMENT STATE SALES TAX

$\$ 368,198.90$

TOTAL CURRENT AMOUNT

$\$ 387,175.87$

ARREARS AMOUNT

$\$ 403.754 .44$

TOTAL AMOUNT DUE 
ACCOUNT

2325-2001-1

CONTRACT NO.

FO2601-79-DOO23
DATE OF BILL: December 30, 1996

DATE DELINQUENT: January 10, 1997

\section{TUCSON ELECTRIC POWER COMPANY SERVICES RENDERED}

U. S. A. F. DAVIS MONTHAN AIR FORCE BASE NOVEMBER 25, 1996 TO DECEMBER 26, 1996

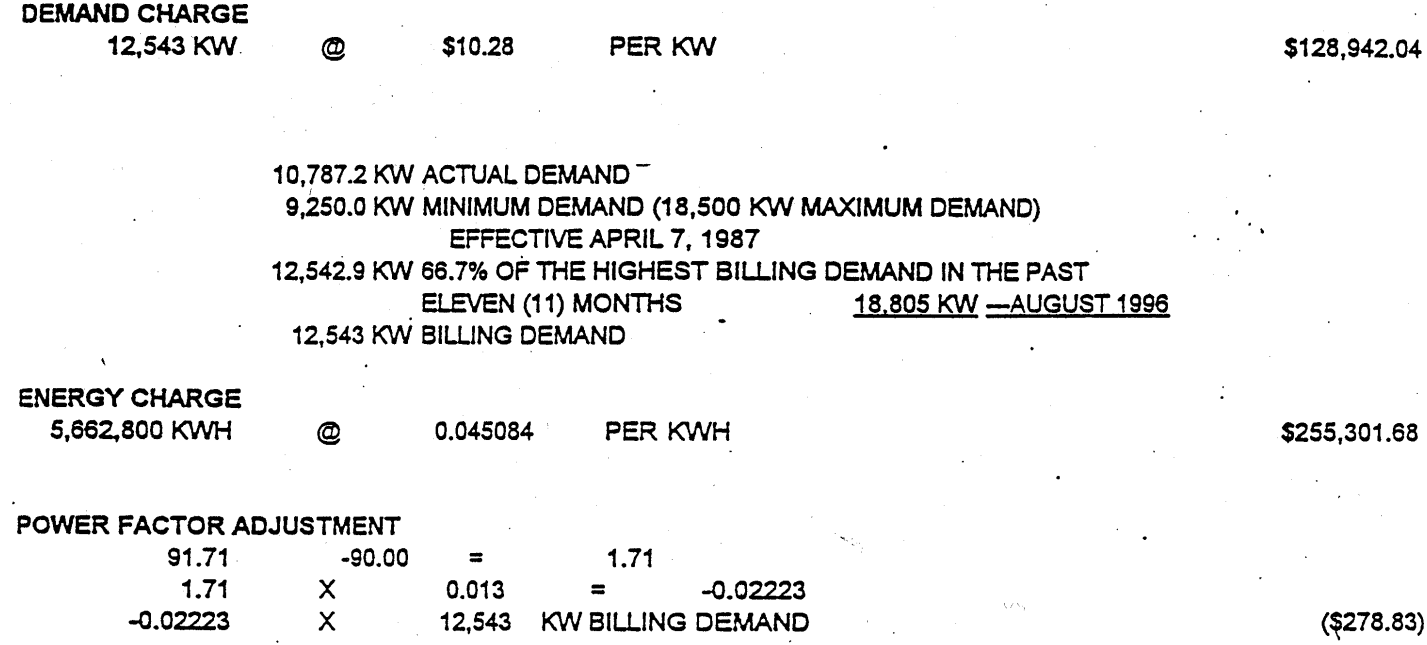

TOTAL CURRENT AMOUNT 


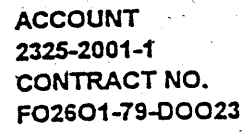

U. S. A. F. DAVIS MONTHAN AIR FORCE BASE OCTOBER 24, 1996 TO NOVEMBER 25, 1996

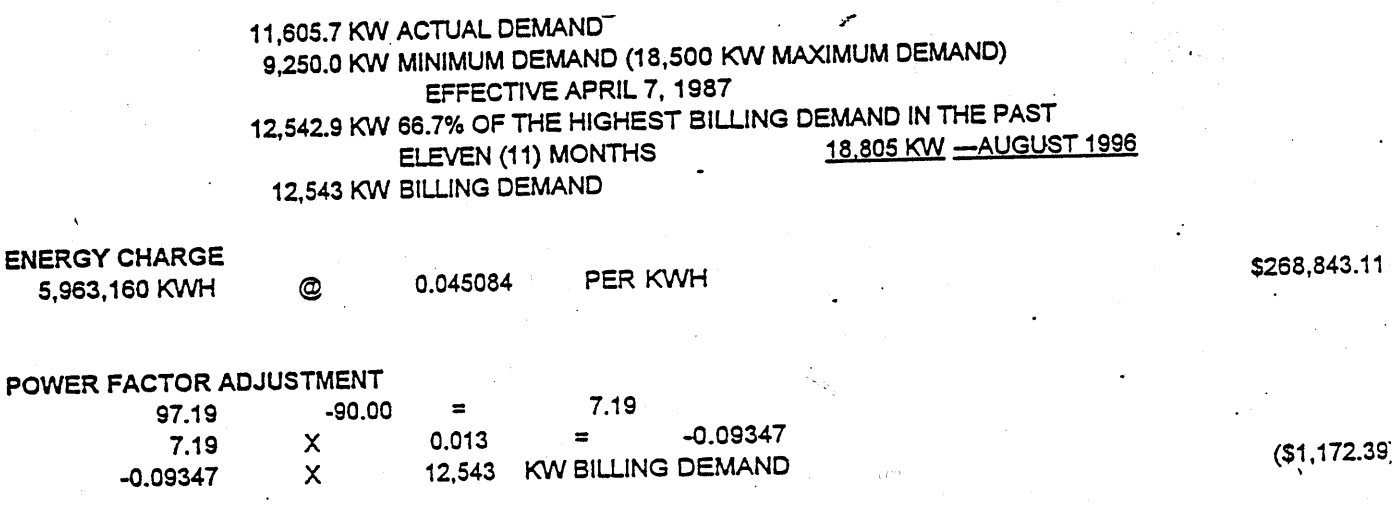

$\$ 268,843.11$

$(\$ 1,172.39$

$\$ 128,942.04$

SUBTOTAL

TOTAL CURRENT AMOUNT 


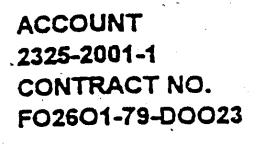

ELEVEN (11) MONTHS - $18,805 \mathrm{KW}=$ AUGUST 1996

DATE OF BILL: October 28, 1996 DATE DEUNQUENT: November 8, 1996

\section{TUCSON ELECTRIC POWER COMPANY SERVICES RENDERED}

U. S. A. F. DAVIS MONTHAN AIR FORCE BASE SEPTEMBER 25, 1996 TO OCTOBER 24, 1996

$-0.06448$

$x$

$\$ 174,215.16$ 


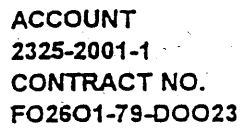

DATE OF BILL: August 28, 1996 DATE DELINQUENT: September 11, 1996

\section{TUCSON ELECTRIC POWER COMPANY SERVICES RENDERED}

U. S. A. F. DAVIS MONTHAN AIR FORCE BASE JULY 25, 1996 TO AUGUST 26, 1996

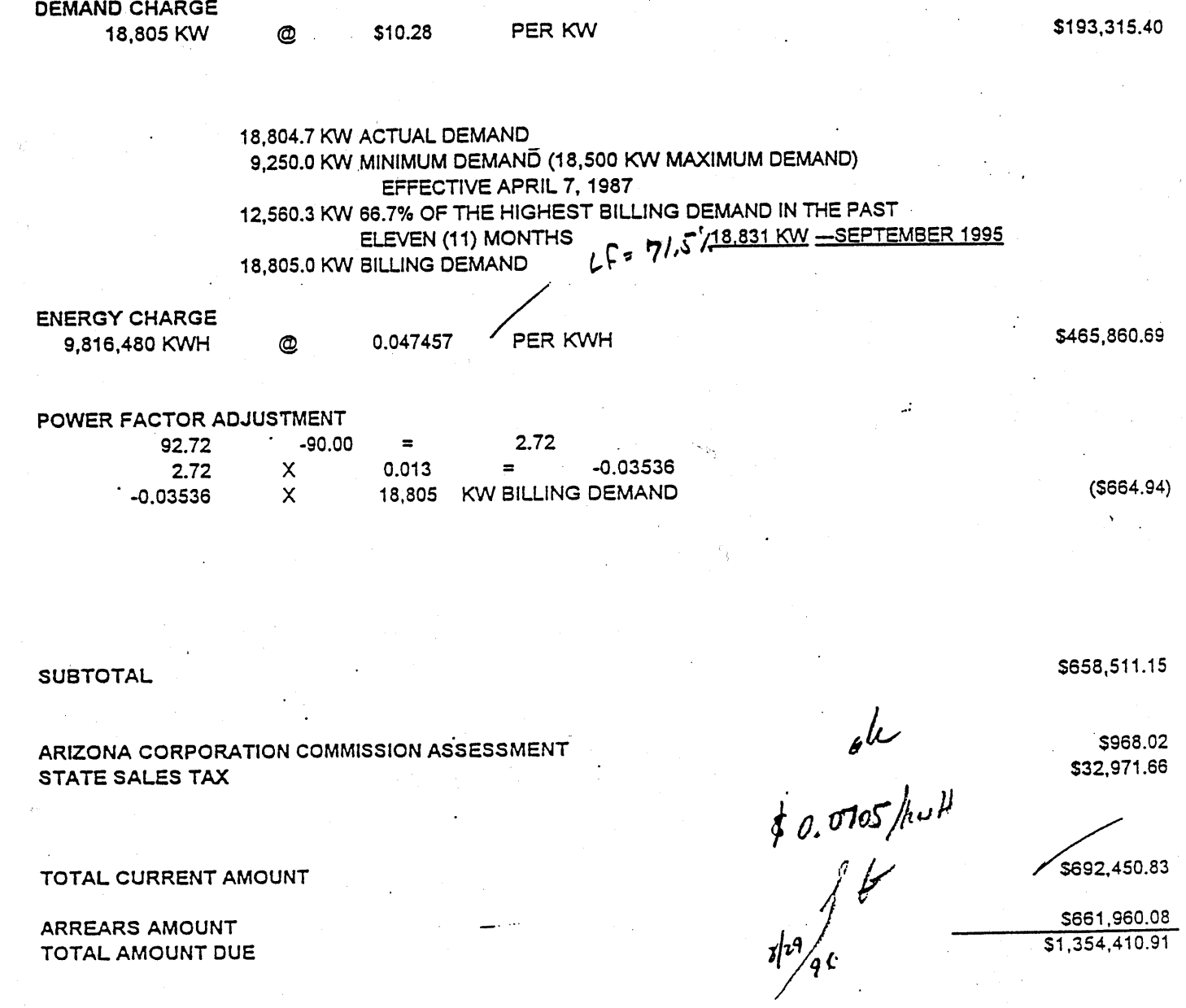




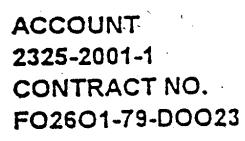

$\begin{array}{lll}\$ 10.28 & \text { PER KW } \$ 191,814.5\end{array}$

$\begin{array}{lll}\$ 10.28 & \text { PER KW } \$ 191,814.5\end{array}$

\section{TUCSON ELECTRIC POWER COMPANY SERVICES RENDERED}

U. S. A. F. DAVIS MONTHAN AIR FORCE BASE JUNE 25, 1996 TO JULY 25, 1996

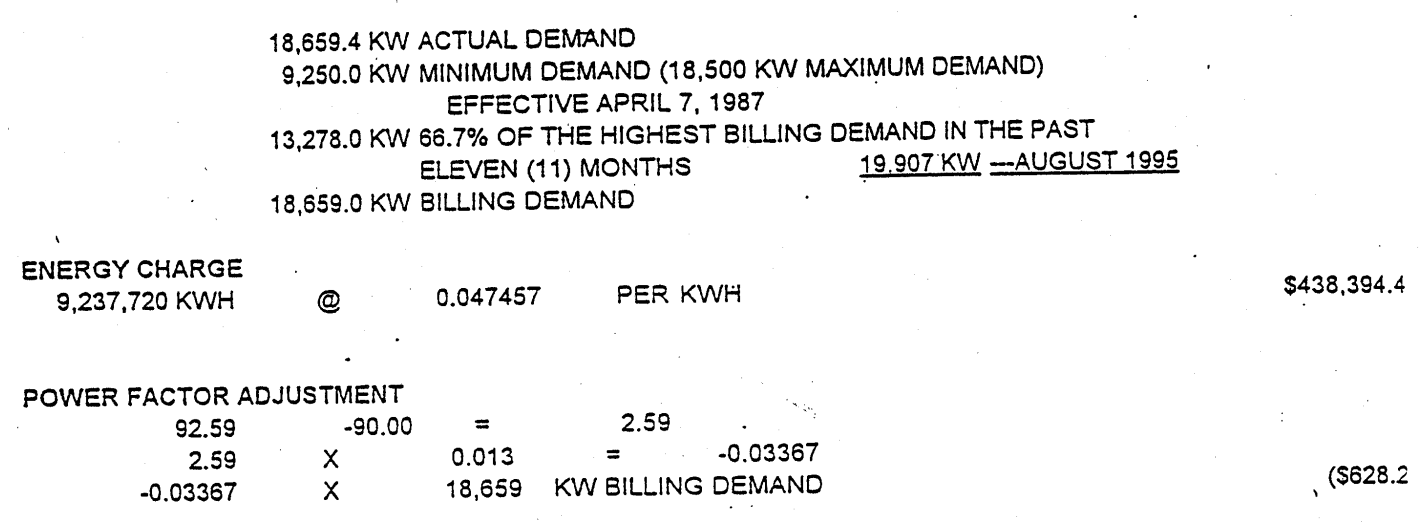

SUBTOTAL

ARIZONA CORPORATION COMMISSION ASSSESSMENT

STATE SALES TAX

TOTAL CURRENT AMOUNT

ARREARS AMOUNT

TOTAL AMOUNT DUE

$\$ 859.3$

$\$ 31,519 . \$$

$\$ 661,960$.

5647,774

$\$ 1,309,734$. 


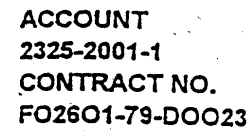

DATE OF BILL: June 27, 1996 DATE DELINQUENT: July 11, 1996

\section{TUCSON ELECTRIC POWER COMPANY SERVICES RENDERED}

U. S. A. F. DAVIS MONTHAN AIR FORCE BASE MAY 24, 1996 TO JUNE 25, 1996

$-0.04121$

$\begin{array}{ccc}= & 3.17 & 0 \\ 0.013 & = & -0.04121 \\ 18,344 & \text { KW } & \text { BILLING DEMAND }\end{array}$

$(\$ 755.96)$

UBTOTAL

$\$ 616,089.41$

RIZONA CORPORATION COMMISSION ASSESSMENT TATE SALES TAX

$\cdot$

JTAL CURRENT AMOUNT

$\$ 647,774.90$

RREARS AMOUNT

$\$ 575,399.82$

JTAL AMOUNT DUE 


\section{Appendix D: Spreadsheet Calculation Based on Option \#2a}

Historic

\begin{tabular}{|c|c|c|c|c|c|}
\hline & & & $\begin{array}{l}\text { Operating } 3 \text { of } \\
\text { Operating } 3 \text { of }\end{array}$ & $\begin{array}{l}\text { nths (Sum } \\
\text { nths (Win }\end{array}$ & \\
\hline Month & $\begin{array}{l}\text { Billing } \\
\text { Demand }\end{array}$ & $\begin{array}{l}\text { Actual } \\
\text { Demand }\end{array}$ & Month & $\begin{array}{l}\text { Billing } \\
\text { Demand }\end{array}$ & $\begin{array}{l}\text { Actual } \\
\text { Demand }\end{array}$ \\
\hline JUL 96 (S) & 18,659 & 18,659 & +JUL 98 (S) & 18,104 & 18,104 \\
\hline AUG 96(S) & 18,805 & 18,805 & +AUG 98 (S) & 18,250 & 18,250 \\
\hline *SEP 96(S) & 17,876 & 17,876 & ++SEP 98 (S) & 17,876 & 17,876 \\
\hline OCT $96(S)$ & 16,947 & 16,947 & +OCT 98 (S) & 16,392 & 16,392 \\
\hline NOV 96(W) & 12,543 & 11,606 & $++\operatorname{NOV} 98(\mathrm{~W})$ & 12,173 & 11,606 \\
\hline DEC 96(W) & 12,543 & 10,787 & +DEC $98(\mathrm{~W})$ & 12,173 & 10,232 \\
\hline JAN 97 (W) & 12,543 & 11,141 & ++ JAN $99(\mathrm{~W})$ & 12,173 & 11,141 \\
\hline FEB $97(\mathrm{~W})$ & 12,543 & 10,696 & +FEB $99(\mathrm{~W})$ & 12,173 & 10,141 \\
\hline MAR 97(W) & 12,613 & 12,613 & ++MAR $99($ W) & 12,613 & 12,613 \\
\hline APR $97(\mathrm{~W})$ & 14,142 & 14,142 & + APR $99(\mathrm{~W})$ & 13,587 & 13,587 \\
\hline MAY 97 (S) & 17,026 & 17,026 & ++MAY 99 (S) & 17,026 & 17,026 \\
\hline JUN 97 (S) & 18,099 & 18,099 & ++JUN 99 (S) & 18,099 & 18,099 \\
\hline
\end{tabular}

\begin{tabular}{|c|c|c|}
\hline \multicolumn{2}{|c|}{ SAVINGS } & \multirow{2}{*}{$\begin{array}{l}\text { RECAP FOR SEASON } \\
\text { Summer: }\end{array}$} \\
\hline JUL & $555 \mathrm{~kW}$ & \\
\hline$A \cup G$ & $555 \mathrm{~kW}$ & 3 months @ 555 kW reduction each \\
\hline SEP & $0 \mathrm{~kW}$ & 3 months @ 0 kW reduction each \\
\hline OCT & $555 \mathrm{~kW}$ & Winter: \\
\hline NOV & $370 \mathrm{~kW}$ & 4 months @ 370 kW reduction each \\
\hline DEC & $370 \mathrm{~kW}$ & 1 month @ 555 kW reduction each \\
\hline JAN & $370 \mathrm{~kW}$ & 1 month @ 0 kW reduction each \\
\hline FEB & $370 \mathrm{~kW}$ & \\
\hline MAR & $0 \mathrm{~kW}$ & \\
\hline APR & $555 \mathrm{~kW}$ & \\
\hline MAY & $0 \mathrm{~kW}$ & \\
\hline JUN & $0 \mathrm{~kW}$ & \\
\hline
\end{tabular}

"*” indicates data that has been averaged between the month preceding and that following.

"+" symbol indicates a month in which the engine-generator operated continuously,

"++" indicates a month when it did not.

(S) denotes a Summer month, while (W) denotes a Winter month. 


\section{Appendix E: Spreadsheet Calculation Based on Option \#2b}

Historic

With Engine Generator

Operating 3 of 6 Months (Summer)

Operating 3 of 6 Months (Winter)

\begin{tabular}{|l|l|l|l|l|l|}
\hline Month & $\begin{array}{l}\text { Billing } \\
\text { Demand }\end{array}$ & $\begin{array}{l}\text { Actual } \\
\text { Demand }\end{array}$ & Month & $\begin{array}{l}\text { Billing } \\
\text { Demand }\end{array}$ & $\begin{array}{l}\text { Actual } \\
\text { Demand }\end{array}$ \\
\hline JUL 96 $(\mathrm{S})$ & 18,659 & 18,659 & +JUL 98 (S) & 17,900 & 17,900 \\
\hline AUG 96(S) & 18,805 & 18,805 & +AUG 98 (S) & 18,046 & 18,046 \\
\hline *SEP 96(S) & 17,876 & 17,876 & ++SEP 98 (S) & 17,876 & 17,876 \\
\hline OCT 96 (S) & 16,947 & 16,947 & +OCT 98 (S) & 16,188 & 16,188 \\
\hline NOV 96(W) & 12,543 & 11,606 & ++NOV 98 (W) & 12,037 & 11,606 \\
\hline DEC 96(W) & 12,543 & 10,787 & +DEC 98 (W) & 12,037 & 10,028 \\
\hline JAN 97 (W) & 12,543 & 11,141 & ++JAN 99 (W) & 12,037 & 11,141 \\
\hline FEB 97 (W) & 12,543 & 10,696 & +FEB 99 (W) & 12,037 & 9,937 \\
\hline MAR 97(W) & 12,613 & 12,613 & ++MAR 99 $(\mathrm{W})$ & 12,613 & 12,613 \\
\hline APR 97 (W) & 14,142 & 14,142 & +APR 99 (W) & 13,383 & 13,383 \\
\hline MAY 97 (S) & 17,026 & 17,026 & ++MAY 99 (S) & 17,026 & 17,026 \\
\hline JUN 97 (S) & 18,099 & 18,099 & ++JUN 99 (S) & 18,099 & 18,099 \\
\hline
\end{tabular}

\begin{tabular}{|l|c|l|}
\hline \multicolumn{2}{|l|}{ SAVINGS } & RECAP FOR SEASON \\
\hline JUL & $759 \mathrm{~kW}$ & Summer: \\
\hline AUG & $759 \mathrm{~kW}$ & 3 months @ $759 \mathrm{~kW}$ reduction each \\
\hline SEP & $0 \mathrm{~kW}$ & 3 months @ $0 \mathrm{~kW}$ reduction each \\
\hline OCT & $759 \mathrm{~kW}$ & Winter: \\
\hline NOV & $506 \mathrm{~kW}$ & 4 months @ $506 \mathrm{~kW}$ reduction each \\
\hline DEC & $506 \mathrm{~kW}$ & 1 month @ 759 kW reduction each \\
\hline JAN & $506 \mathrm{~kW}$ & 1 month @ $0 \mathrm{~kW}$ reduction each \\
\hline FEB & $506 \mathrm{~kW}$ & \\
\hline MAR & $0 \mathrm{~kW}$ & \\
\hline APR & $759 \mathrm{~kW}$ & \\
\hline MAY & $0 \mathrm{~kW}$ & \\
\hline JUN & $0 \mathrm{~kW}$ & \\
"*” indicates data that has been averaged between the month preceding
\end{tabular}

indicates data that has been averaged between the month preceding and that following.

"+" symbol indicates a month in which the engine-generator operated continuously,

"++" indicates a month when it did not.

(S) denotes a Summer month,

(W) denotes a Winter month.

( )-number within = \#units operating continuously for that month 


\section{Appendix F: Spreadsheet Calculation Based on Options \#2c and 3a}

Historic
\begin{tabular}{|l|l|l|l|l|l|}
\hline Month & $\begin{array}{l}\text { Billing } \\
\text { Demand }\end{array}$ & $\begin{array}{l}\text { Actual } \\
\text { Demand }\end{array}$ & Month & $\begin{array}{l}\text { Billing } \\
\text { Demand }\end{array}$ & $\begin{array}{l}\text { Actual } \\
\text { Demand }\end{array}$ \\
\hline JUL $96(\mathrm{~S})$ & 18,659 & 18,659 & JUL 98 (S) & 17,141 & $17,141(2)$ \\
\hline AUG 96(S) & 18,805 & 18,805 & AUG 98 (S) & 17,287 & $17,287(2)$ \\
\hline${ }^{*}$ SEP 96(S) & 17,876 & 17,876 & SEP 98 (S) & 17,117 & $17,117(1)$ \\
\hline OCT 96 (S) & 16,947 & 16,947 & OCT 98 (S) & 15,429 & $15,429(2)$ \\
\hline NOV 96(W) & 12,543 & 11,606 & NOV 98 (W) & 11,530 & $10,847(1)$ \\
\hline DEC 96(W) & 12,543 & 10,787 & DEC 98 (W) & 11,530 & $9,269(2)$ \\
\hline JAN 97 (W) & 12,543 & 11,141 & JAN 99 (W) & 11,530 & $10,382(1)$ \\
\hline FEB 97 (W) & 12,543 & 10,696 & FEB 99 $(W)$ & 11,530 & $9,178(2)$ \\
\hline MAR 97(W) & 12,613 & 12,613 & MAR 99 (W) & 11,854 & $11,854(1)$ \\
\hline APR 97 (W) & 14,142 & 14,142 & APR 99 (W) & 12,624 & $12,624(2)$ \\
\hline MAY 97 (S) & 17,026 & 17,026 & MAY 99 (S) & 16,267 & $16,267(1)$ \\
\hline JUN 97 (S) & 18,099 & 18,099 & JUN 99 (S) & 17,340 & $17,340(1)$ \\
\hline
\end{tabular}

\begin{tabular}{|l|c|l|}
\hline \multicolumn{2}{|l|}{ SAVINGS } & RECAP FOR SEASON \\
\hline JUL & $1,518 \mathrm{~kW}$ & Summer: \\
\hline AUG & $1,518 \mathrm{~kW}$ & 3 months @ $1,518 \mathrm{~kW}$ reduction each \\
\hline SEP & $759 \mathrm{~kW}$ & 3 months @ $759 \mathrm{~kW}$ reduction each \\
\hline OCT & $1,518 \mathrm{~kW}$ & Winter: \\
\hline NOV & $1,013 \mathrm{~kW}$ & 4 months @ $1,013 \mathrm{~kW}$ reduction each \\
\hline DEC & $1,013 \mathrm{~kW}$ & 1 month @ $759 \mathrm{~kW}$ reduction each \\
\hline JAN & $1,013 \mathrm{~kW}$ & 1 month @ $1,518 \mathrm{~kW}$ reduction each \\
\hline FEB & $1,013 \mathrm{~kW}$ & \\
\hline MAR & $759 \mathrm{~kW}$ & \\
\hline APR & $1,518 \mathrm{~kW}$ & \\
\hline MAY & $759 \mathrm{~kW}$ & \\
\hline JUN & $759 \mathrm{~kW}$ & \\
\hline
\end{tabular}

(S) denotes a Summer month

(W) denotes a Winter month

( )-number within = \#units operating continuously for that month 


\section{Appendix G: Spreadsheet Calculation Based on Options \#2d and 3c}

Historic Month \begin{tabular}{|l} 
JUL $96(1$ \\
\hline$A U G 96$
\end{tabular} AUG 96(S) *SEP 96(S) OCT $96(\mathrm{~S})$ NOV 96(W) DEC 96(W) JAN $97(\mathrm{~W})$ FEB $97(\mathrm{~W})$ MAR 97(W) APR $97(\mathrm{~W})$ MAY $97(\mathrm{~S})$ JUN $97(\mathrm{~S})$

(S)

\begin{tabular}{|c|c|}
\hline $\begin{array}{l}\text { Billing } \\
\text { Demand }\end{array}$ & $\begin{array}{l}\text { Actual } \\
\text { Demand }\end{array}$ \\
\hline 18,659 & 18,659 \\
\hline 18,805 & 18,805 \\
\hline 17,876 & 17,876 \\
\hline 16,947 & 16,947 \\
\hline 12,543 & 11,606 \\
\hline 12,543 & 10,787 \\
\hline 12,543 & 11,141 \\
\hline 12,543 & 10,696 \\
\hline 12,613 & 12,613 \\
\hline 14,142 & 14,142 \\
\hline 17,026 & 17,026 \\
\hline 18,099 & 18,099 \\
\hline
\end{tabular}

With Engine Generator

\begin{tabular}{|l|l|ll|}
\hline Month & $\begin{array}{l}\text { Billing } \\
\text { Demand }\end{array}$ & \multicolumn{2}{|l|}{$\begin{array}{l}\text { Actual } \\
\text { Demand }\end{array}$} \\
\hline JUL 98 (S) & 16,382 & $16,382 \quad(3)$ \\
\hline AUG 98 (S) & 16,528 & $16,528 \quad(3)$ \\
\hline SEP 98 (S) & 16,358 & $16,358 \quad(2)$ \\
\hline OCT 98 (S) & 15,429 & 15,429 & $(2)$ \\
\hline NOV 98 (W) & 11,024 & 9,329 & $(3)$ \\
\hline DEC 98 (W) & 11,024 & 9,269 & $(2)$ \\
\hline JAN 99 (W) & 11,024 & 9,623 & $(2)$ \\
\hline FEB 99 (W) & 11,024 & 9,178 & $(2)$ \\
\hline MAR 99 (W) & 11,095 & 11,095 & $(2)$ \\
\hline APR 99 (W) & 11,865 & 11,865 & $(3)$ \\
\hline MAY 99 (S) & 15,508 & $15,508 \quad(2)$ \\
\hline JUN 99 (S) & 16,581 & $16,581 \quad(2)$ \\
\hline
\end{tabular}

\begin{tabular}{|c|c|c|}
\hline \multicolumn{2}{|c|}{ SAVINGS } & \multirow{2}{*}{$\begin{array}{l}\text { RECAP FOR SEASON } \\
\text { Summer: }\end{array}$} \\
\hline JUL & $2,277 \mathrm{~kW}$ & \\
\hline AUG & $2,277 \mathrm{~kW}$ & 4 months@ @ 1,518 kW reduction each \\
\hline SEP & $1,518 \mathrm{~kW}$ & 2 months @ 2,277 kW reduction each \\
\hline OCT & $1,518 \mathrm{~kW}$ & Winter: \\
\hline NOV & $1,519 \mathrm{~kW}$ & 4 months @ 1,519 kW reduction each \\
\hline DEC & $1,519 \mathrm{~kW}$ & 1 month @ 2,277 kW reduction each \\
\hline JAN & $1,519 \mathrm{~kW}$ & 1 month @ 1,518 kW reduction each \\
\hline FEB & $1,519 \mathrm{~kW}$ & \\
\hline MAR & $1,518 \mathrm{~kW}$ & \\
\hline APR & $2,277 \mathrm{~kW}$ & \\
\hline MAY & $1,518 \mathrm{~kW}$ & \\
\hline JUN & $1,518 \mathrm{~kW}$ & \\
\hline
\end{tabular}

"*” indicates data that has been averaged between the month preceding and that following.

(S) denotes a Summer month

(W) denotes a Winter month

( ) -number within = \#units operating continuously for that month 


\section{Appendix H: Spreadsheet Calculation Based on Option \#3b}

Historic
\begin{tabular}{|l|l|l|l|l|l|}
\hline Month & $\begin{array}{l}\text { Billing } \\
\text { Demand }\end{array}$ & $\begin{array}{l}\text { Actual } \\
\text { Demand }\end{array}$ & Month & $\begin{array}{l}\text { Billing } \\
\text { Demand }\end{array}$ & $\begin{array}{l}\text { Actual } \\
\text { Demand }\end{array}$ \\
\hline JUL 96 $(\mathrm{S})$ & 18,659 & 18,659 & JUL 98 (S) & 15,623 & $15,623(4)$ \\
\hline AUG 96(S) & 18,805 & 18,805 & AUG 98 (S) & 15,769 & $15,769(4)$ \\
\hline${ }^{*}$ SEP 96(S) & 17,876 & 17,876 & SEP 98 (S) & 15,599 & $15,599(3)$ \\
\hline OCT 96 (S) & 16,947 & 16,947 & OCT 98 (S) & 14,670 & $14,670(3)$ \\
\hline NOV 96(W) & 12,543 & 11,606 & NOV 98 (W) & 10,518 & $8,570(4)$ \\
\hline DEC 96(W) & 12,543 & 10,787 & DEC 98 (W) & 10,518 & $8,510(3)$ \\
\hline JAN 97 (W) & 12,543 & 11,141 & JAN 99 (W) & 10,518 & $8,864(3)$ \\
\hline FEB 97 (W) & 12,543 & 10,696 & FEB 99 (W) & 10,518 & $8,419(3)$ \\
\hline MAR 97(W) & 12,613 & 12,613 & MAR 99 (W) & 10,518 & $10,336(3)$ \\
\hline APR 97 (W) & 14,142 & 14,142 & APR 99 (W) & 11,106 & $11,106(4)$ \\
\hline MAY 97 (S) & 17,026 & 17,026 & MAY 99 (S) & 14,749 & $14,749(3)$ \\
\hline JUN 97 (S) & 18,099 & 18,099 & JUN 99 (S) & 15,822 & $15,822(3)$ \\
\hline
\end{tabular}

\begin{tabular}{|l|l|l|}
\hline \multicolumn{2}{|l|}{ SAVINGS } & RECAP FOR SEASON \\
\hline JUL & $3,036 \mathrm{~kW}$ & Summer: \\
\hline AUG & $3,036 \mathrm{~kW}$ & 4 months @ 2,277 kW reduction each \\
\hline SEP & $2,277 \mathrm{~kW}$ & 2 months @ 3,036 kW reduction each \\
\hline OCT & $2,277 \mathrm{~kW}$ & Winter: \\
\hline NOV & $2,025 \mathrm{~kW}$ & 4 months @ 2,025 kW reduction each \\
\hline DEC & $2,025 \mathrm{~kW}$ & 1 month @ 2,095 kW reduction each \\
\hline JAN & $2,025 \mathrm{~kW}$ & 1 month @ 3,036 kW reduction each \\
\hline FEB & $2,025 \mathrm{~kW}$ & \\
\hline MAR & $2,095 \mathrm{~kW}$ & \\
\hline APR & $3,036 \mathrm{~kW}$ & \\
\hline MAY & $2,277 \mathrm{~kW}$ & \\
\hline JUN & $2,277 \mathrm{~kW}$ & \\
\hline
\end{tabular}

“*” indicates data that's been averaged between the month preceding and that following.

(S) denotes a Summer month

(W) denotes a Winter month

( )-number within = \#units operating continuously for that month 


\section{Appendix I: Spreadsheet Calculation Based on Option \#3d}

Historic
\begin{tabular}{|l|l|l|l|l|l|}
\hline Month & $\begin{array}{l}\text { Billing } \\
\text { Demand }\end{array}$ & $\begin{array}{l}\text { Actual } \\
\text { Demand }\end{array}$ & Month & $\begin{array}{l}\text { Billing } \\
\text { Demand }\end{array}$ & $\begin{array}{l}\text { Actual } \\
\text { Demand }\end{array}$ \\
\hline JUL 96 $(\mathrm{S})$ & 18,659 & 18,659 & JUL 98 (S) & 14,105 & $14,105(6)$ \\
\hline AUG 96(S) & 18,805 & 18,805 & AUG 98 (S) & 14,251 & $14,251(6)$ \\
\hline${ }^{*}$ SEP 96(S) & 17,876 & 17,876 & SEP 98 (S) & 14,081 & $14,081(5)$ \\
\hline OCT 96 (S) & 16,947 & 16,947 & OCT 98 (S) & 13,911 & $13,911(4)$ \\
\hline NOV 96(W) & 12,543 & 11,606 & NOV 98 (W) & 9,505 & $7,052(6)$ \\
\hline DEC 96(W) & 12,543 & 10,787 & DEC 98 (W) & 9,505 & $6,992(5)$ \\
\hline JAN 97 (W) & 12,543 & 11,141 & JAN 99 (W) & 9,505 & $8,105(4)$ \\
\hline FEB 97 (W) & 12,543 & 10,696 & FEB 99 (W) & 9,505 & $6,901(5)$ \\
\hline MAR 97(W) & 12,613 & 12,613 & MAR 99 (W) & 9,577 & $9,577(4)$ \\
\hline APR 97 (W) & 14,142 & 14,142 & APR 99 (W) & 9,588 & $9,588(6)$ \\
\hline MAY 97 (S) & 17,026 & 17,026 & MAY 99 (S) & 13,990 & $13,990(4)$ \\
\hline JUN 97 (S) & 18,099 & 18,099 & JUN 99 (S) & 14,304 & $14,304(5)$ \\
\hline
\end{tabular}

\begin{tabular}{|c|c|c|}
\hline \multicolumn{2}{|c|}{ SAVINGS } & \multirow{2}{*}{$\begin{array}{l}\text { RECAP FOR SEASON } \\
\text { Summer: }\end{array}$} \\
\hline JUL & $4,554 \mathrm{~kW}$ & \\
\hline AUG & $4,554 \mathrm{~kW}$ & 2 months @ 4,554 kW reduction each \\
\hline SEP & $3,795 \mathrm{~kW}$ & 2 months @ 3,795 kW reduction each \\
\hline OCT & $3,036 \mathrm{~kW}$ & 2 months@ @ 3,036 kW reduction each \\
\hline NOV & $4,554 \mathrm{~kW}$ & Winter: \\
\hline DEC & $3,795 \mathrm{~kW}$ & 4 months @ 3,038 kW reduction each \\
\hline JAN & $3,036 \mathrm{~kW}$ & 1 month @ 4,554 kW reduction each \\
\hline FEB & $3,795 \mathrm{~kW}$ & 1 month @ 3,036 kW reduction each \\
\hline MAR & $3,036 \mathrm{~kW}$ & \\
\hline APR & $4,554 \mathrm{~kW}$ & \\
\hline MAY & $3,036 \mathrm{~kW}$ & \\
\hline JUN & $3,795 \mathrm{~kW}$ & \\
\hline
\end{tabular}




\section{Appendix J: Construction Cost Estimates for Each Option}




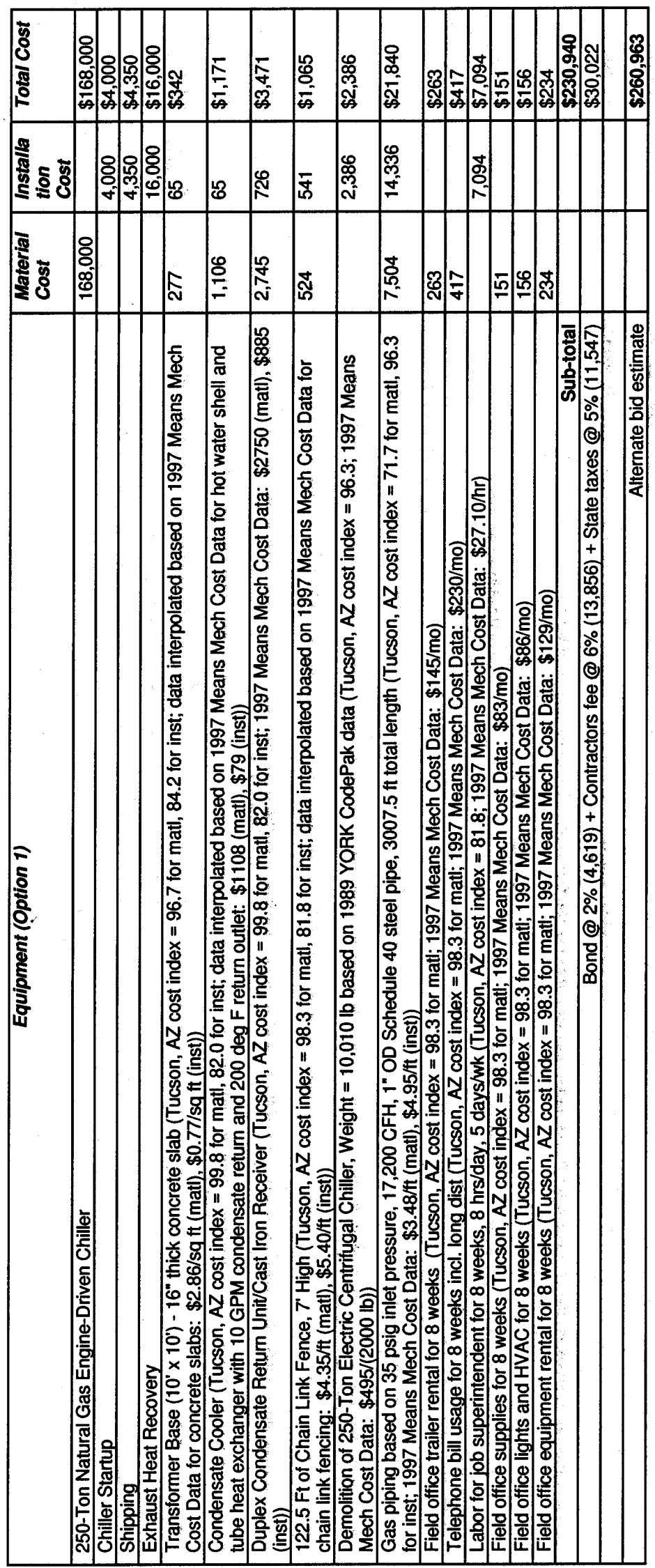




\begin{tabular}{|c|c|c|c|c|c|c|c|c|c|c|c|c|c|}
\hline 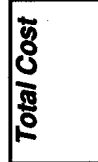 & 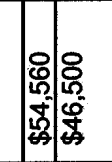 & & & $\bar{E}$ & 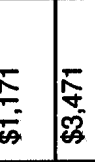 & $\frac{8}{8}$ & 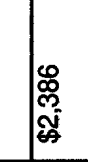 & 导 & 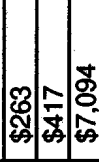 & & & 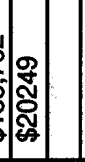 & 당 \\
\hline 衰 & 尽 & $\mid \mathscr{Q}$ & 18 & 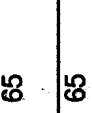 & $\mathbb{N}_{\mathbb{N}}$ & 点 & 黕 & 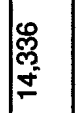 & & & & & \\
\hline$\frac{5}{3}$ & | & & & $\frac{8}{=}$ & $\begin{array}{ll}\stackrel{8}{0} \\
= & \stackrel{g}{N} \\
N\end{array}$ & 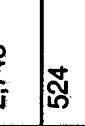 & & 雚 & & $\overline{5}$ & స్ & & \\
\hline . & 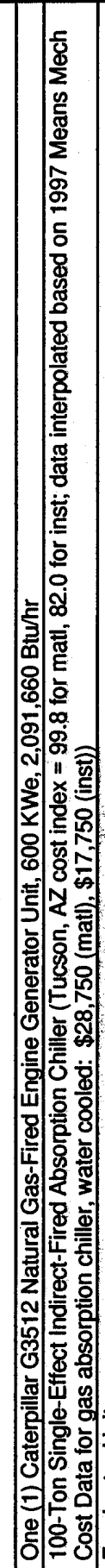 & 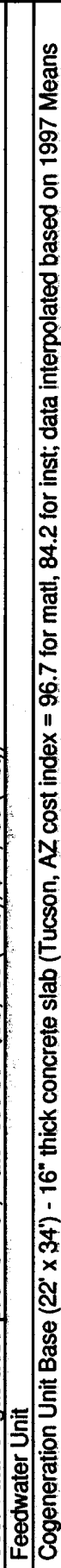 & 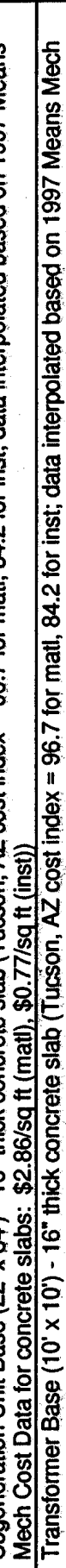 & 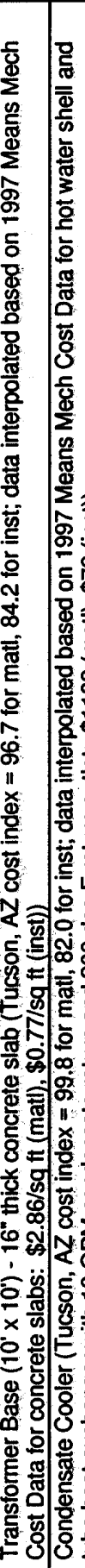 & 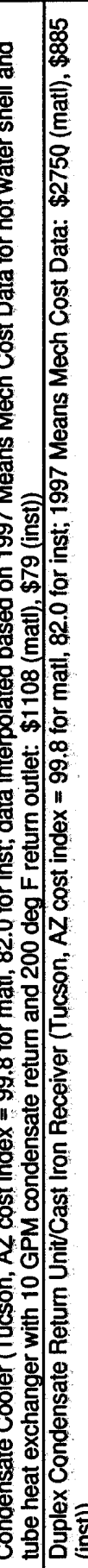 & 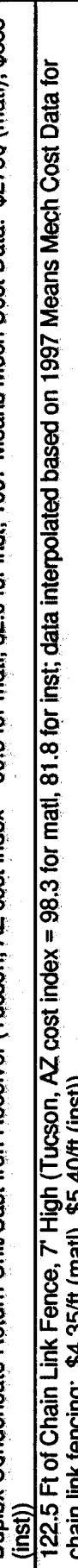 & 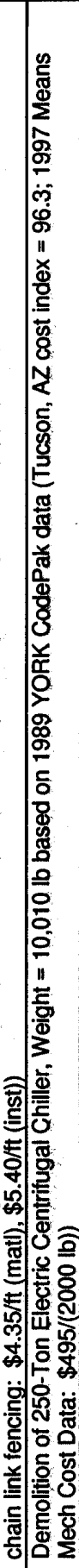 & 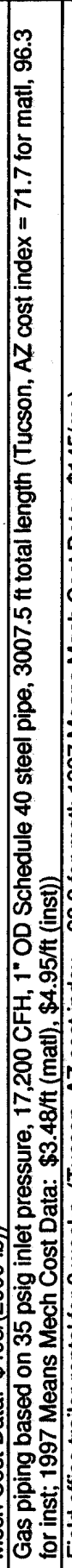 & 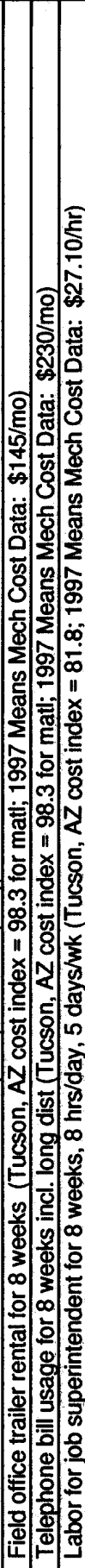 & 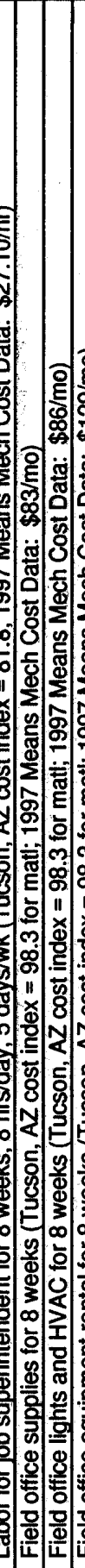 & 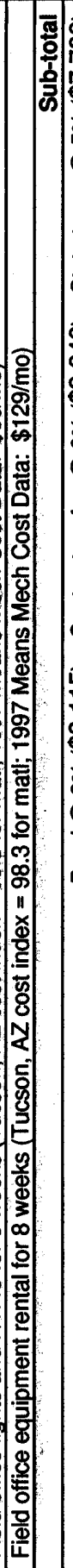 & 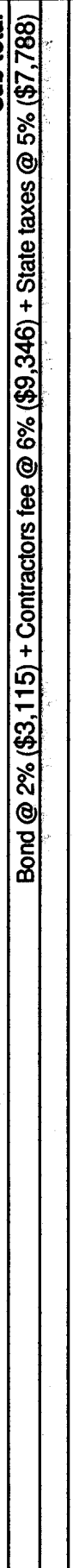 & \\
\hline
\end{tabular}




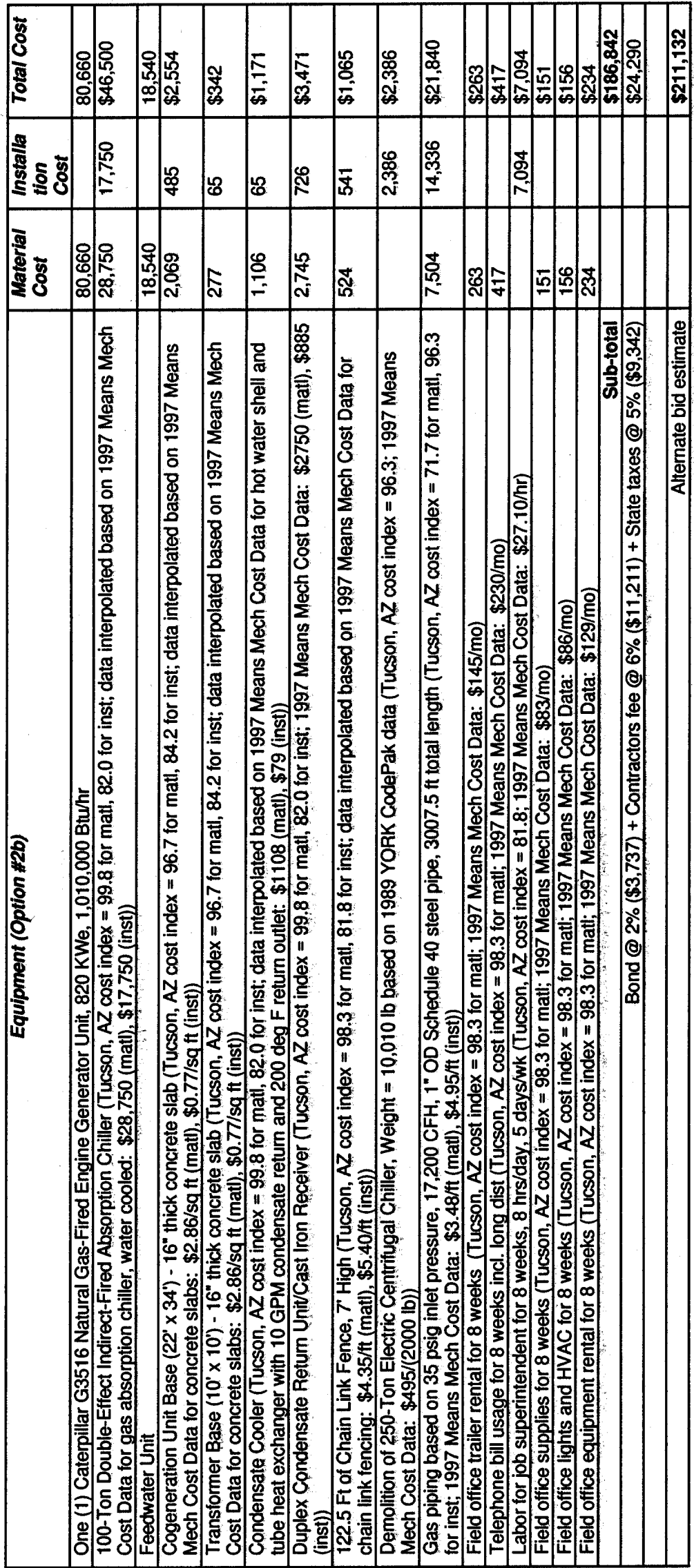




\begin{tabular}{|c|c|c|c|c|c|c|c|c|c|c|c|c|c|c|c|}
\hline | & 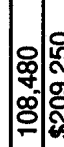 & & : & 芯 & $\bar{E}$ & 蛋 & $\frac{1}{80}$ & 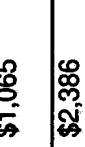 & 趈 & 勇 & 幽 & 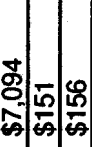 & & & 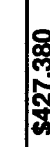 \\
\hline 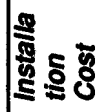 & 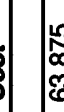 & & 占 & 18 & 18 & $\mid \mathbb{N}$ & 志 & 迈 & 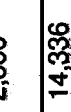 & & & : & & & \\
\hline 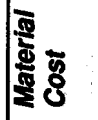 & 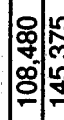 & & & 㓠 & 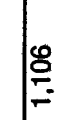 & 战 & 芯 & 诖 & r. & مै. & ֻ & 5 & |̦ & & \\
\hline 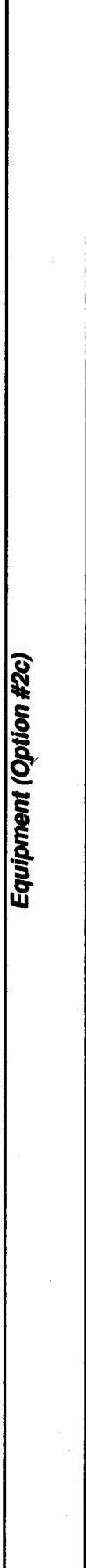 & $\begin{array}{l}0 \\
0 \\
0 \\
0 \\
0\end{array}$ & & 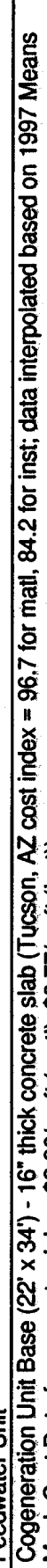 & 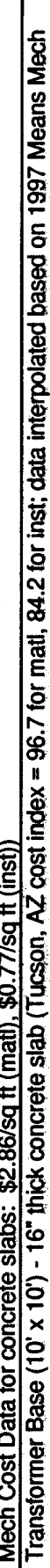 & 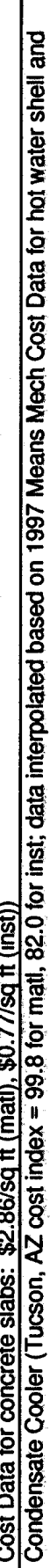 & 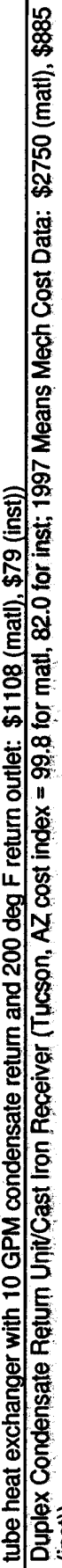 & 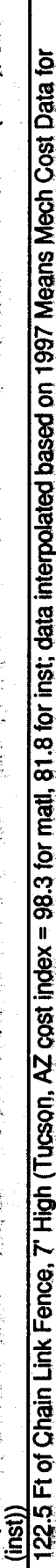 & 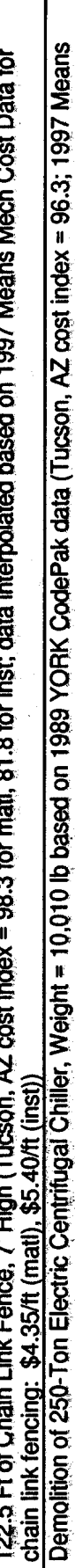 & 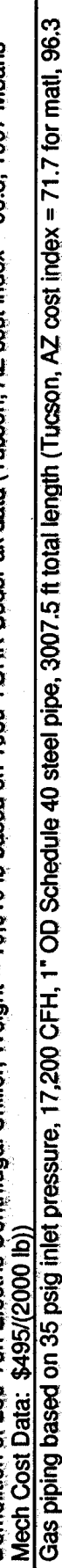 & 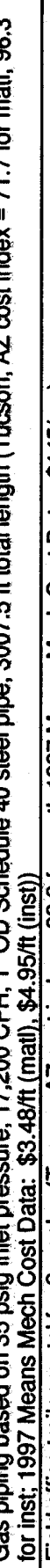 & 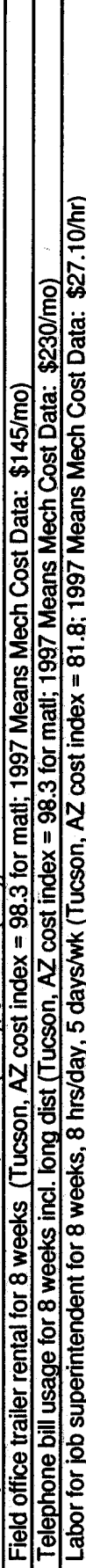 & $\begin{array}{ll} & \\
0 \\
0 \\
0 \\
0 \\
0\end{array}$ & 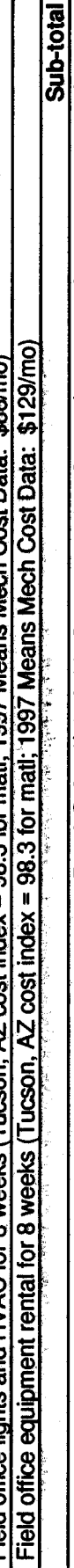 & 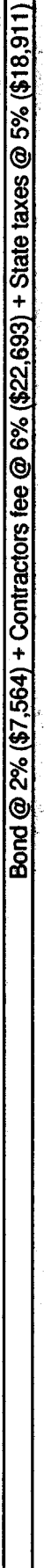 & 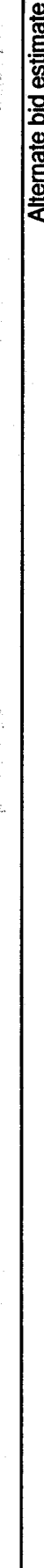 \\
\hline
\end{tabular}




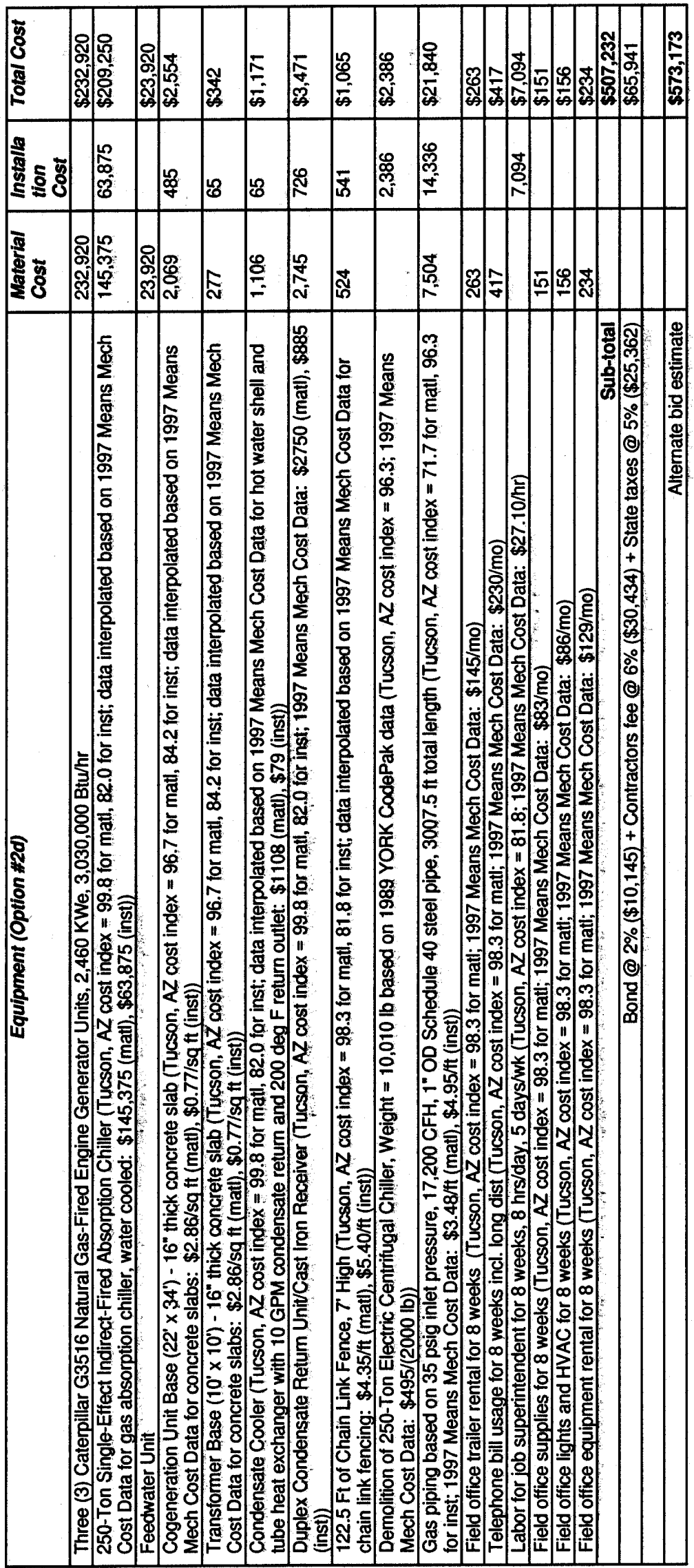




\begin{tabular}{|c|c|c|c|c|c|c|c|c|c|c|c|c|c|c|c|c|}
\hline$\frac{\delta}{8}$ & 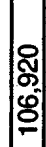 & $\begin{array}{l}8 \\
0 \\
0 \\
0 \\
\$ \\
\$\end{array}$ & & 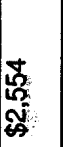 & ঙ્ઝ & 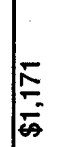 & 委 & $\frac{1}{80}$ & 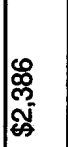 & | & & 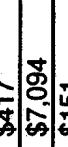 & 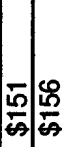 & 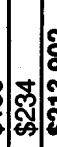 & 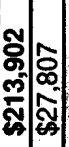 & 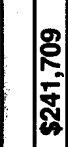 \\
\hline 丞 & & N & & $\stackrel{8}{\circ}$ & 18 & 18 & $\stackrel{N}{N}$ & 芯 & 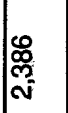 & 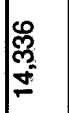 & & 志 & & & & \\
\hline 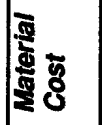 & & 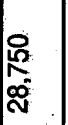 & & $\begin{array}{l}\mathscr{Z} \\
\text { N }\end{array}$ & E & $\frac{8}{-}$ & 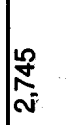 & ฟั๋ & & 䓦 & స্টে & $\overline{8}$ & & న్స్ & & \\
\hline 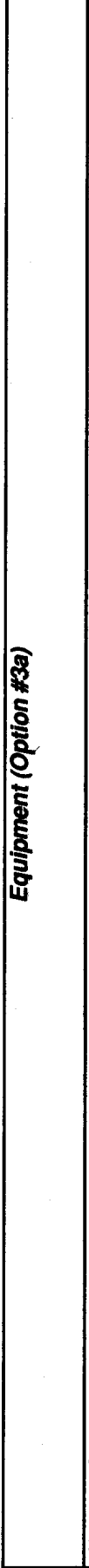 & 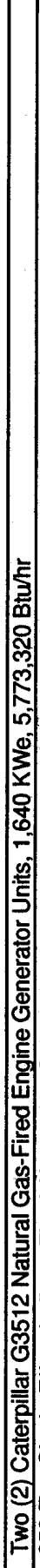 & 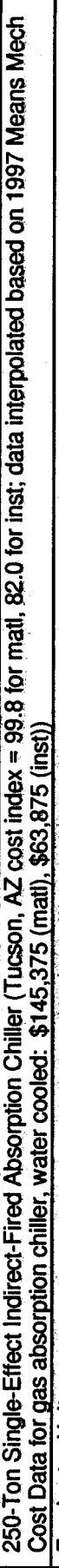 & & 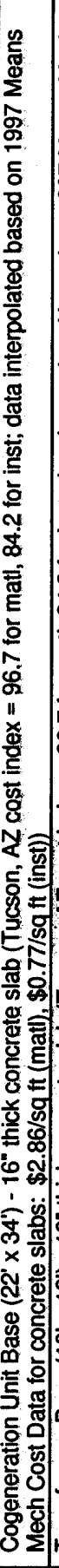 & 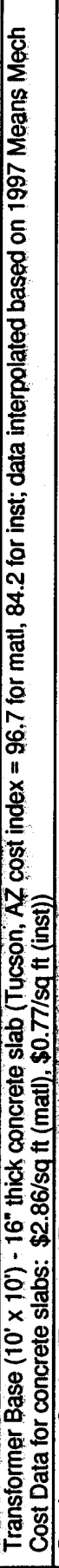 & 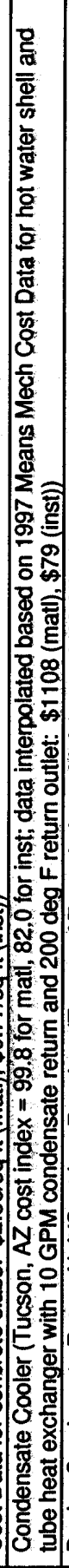 & 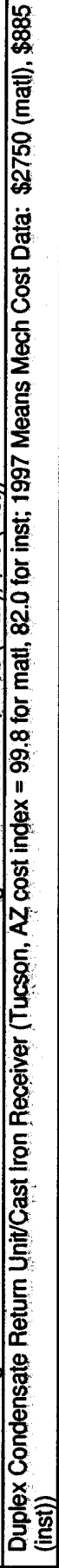 & 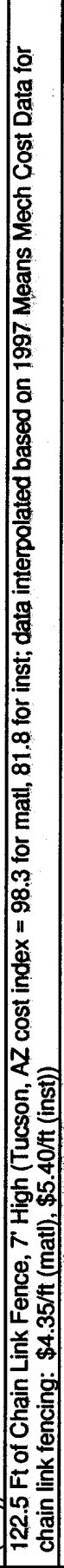 & 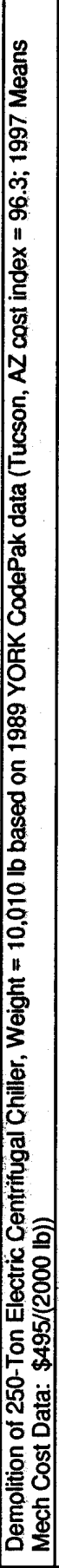 & 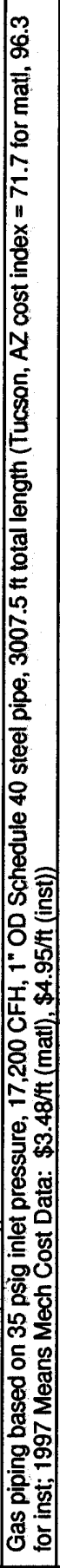 & 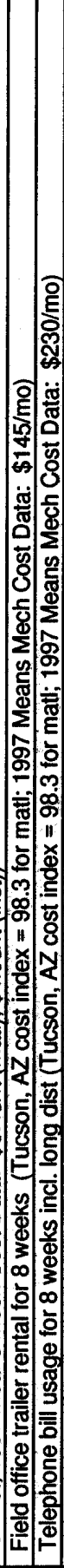 & 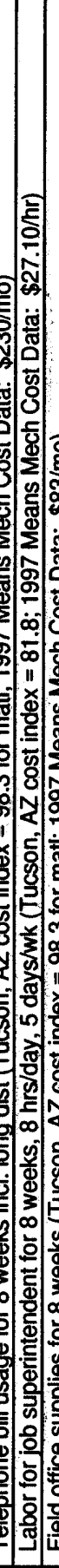 & 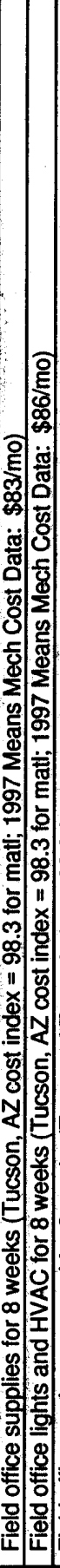 & 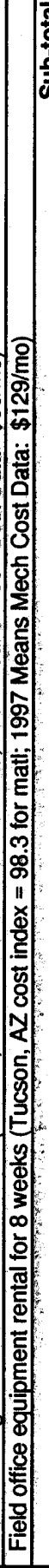 & 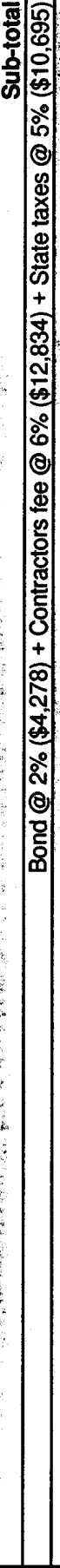 & 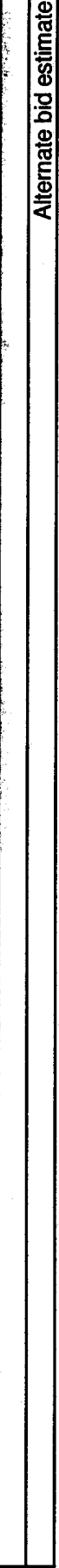 \\
\hline
\end{tabular}




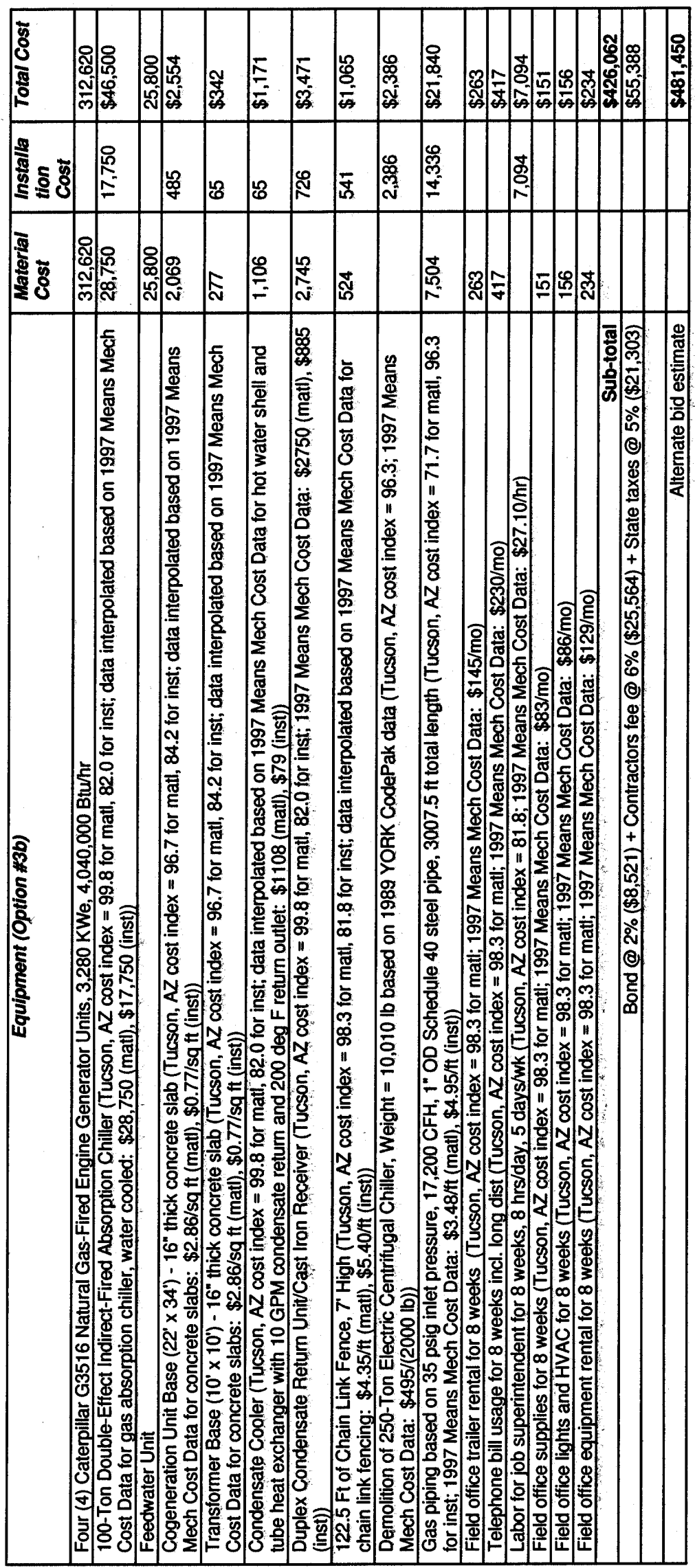




\begin{tabular}{|c|c|c|c|c|c|c|c|c|c|c|c|c|c|}
\hline 造 & 各|윯 & 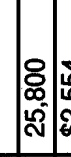 & & 促 & $\underset{\bar{A}}{\bar{A}}$ & 等 & 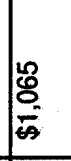 & 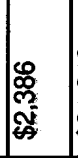 & 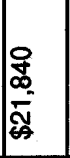 & 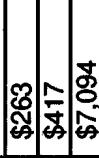 & 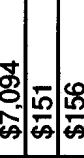 & ค่: & . \\
\hline 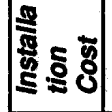 & 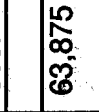 & & $\ddot{8}$ & 18 & 18 & $\mathbb{N}_{\mathbb{N}}$ & 息 & $\mid$ & 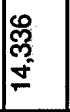 & & | & & \\
\hline 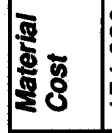 & & & & 战 & $\stackrel{0}{-}$ & $\underset{\substack{f \\
\text { N }}}{\text { s. }}$ & $\mathbb{\pi}$ & & 范 & $\frac{\pi}{4}$ & & ? & \\
\hline 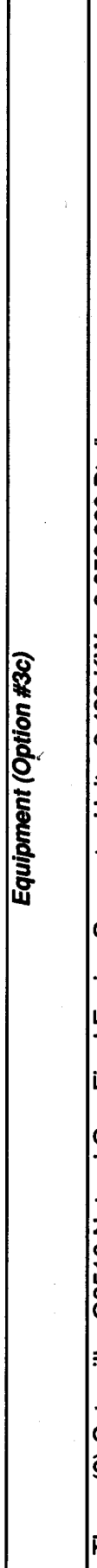 & 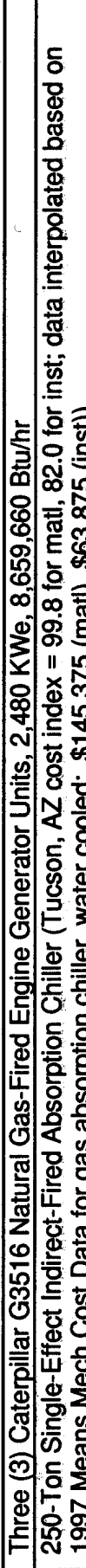 & & 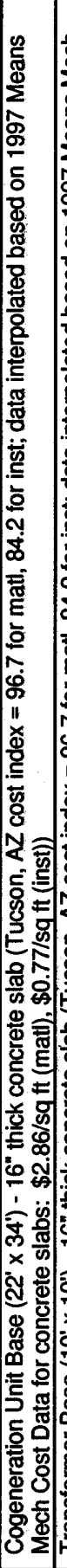 & 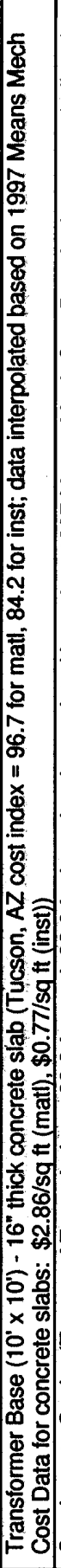 & 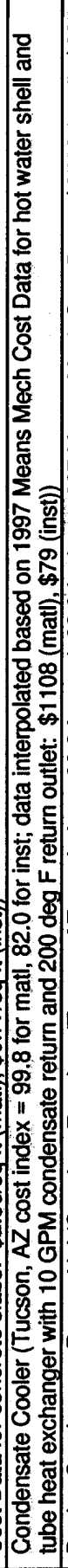 & 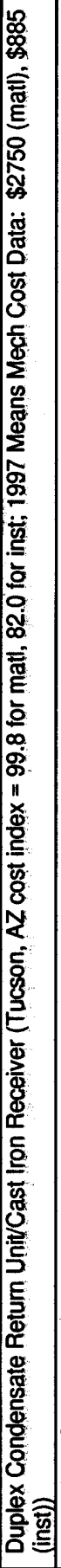 & 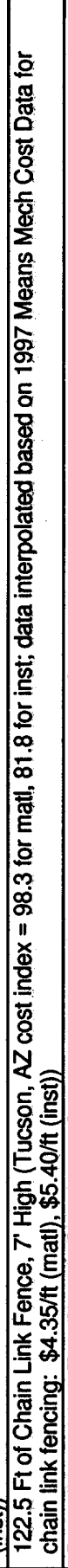 & 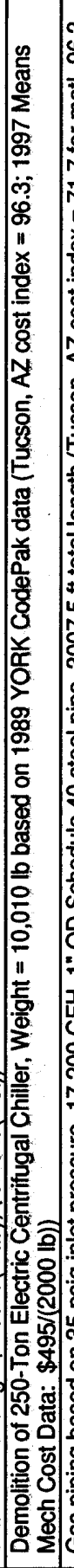 & 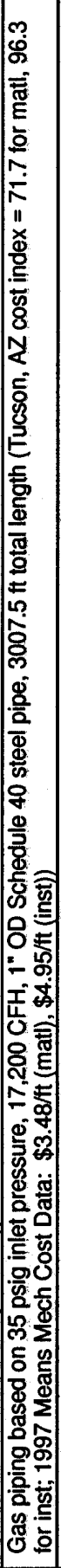 & 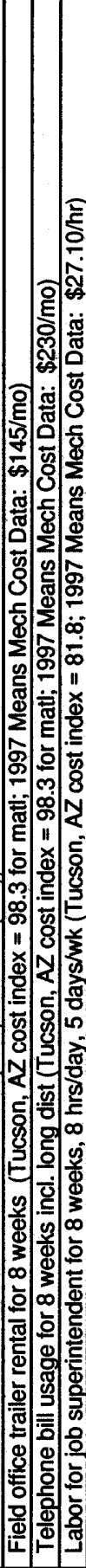 & 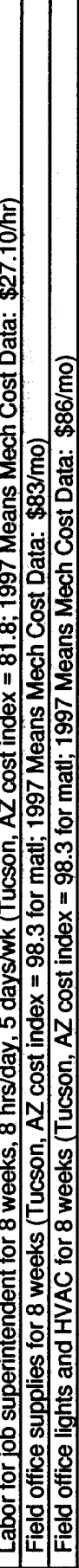 & 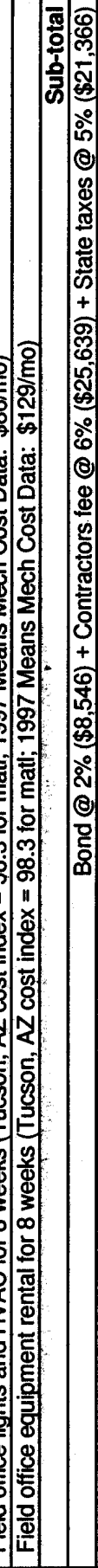 & 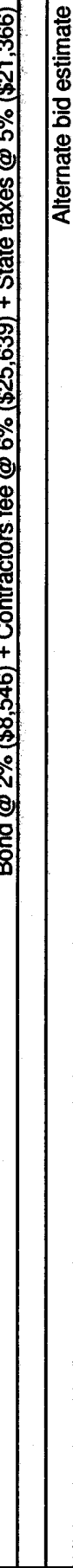 \\
\hline
\end{tabular}




\begin{tabular}{|c|c|c|c|c|c|c|c|c|c|c|c|c|c|c|}
\hline 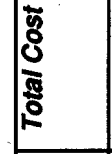 & స్స్తి & & 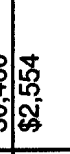 & 芯 & $\frac{\bar{N}}{\bar{N}}$ & $\begin{array}{l}\bar{f} \\
\Re \\
\Re\end{array}$ & 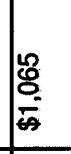 & 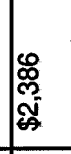 & 高 & 芯 & 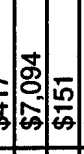 & & 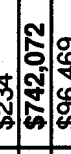 & \\
\hline ș & 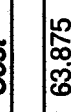 & & 米 & |๕ & ஜ & 蛋 & 昰 & 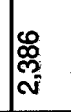 & 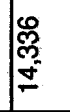 & & 惫 & & & \\
\hline 离 & & & : & 佂 & $\stackrel{\mathscr{1}}{-}$ & 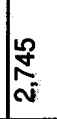 & జี̃ & & 芯 & 禺 & $\overline{5}$ & $\stackrel{n}{\sim}$ & & \\
\hline 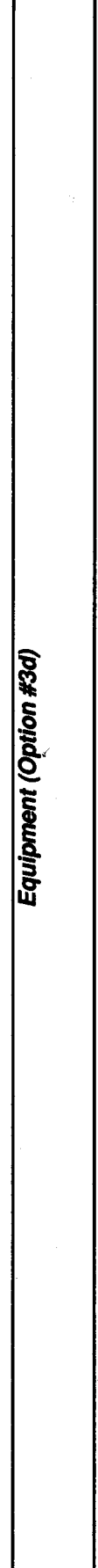 & 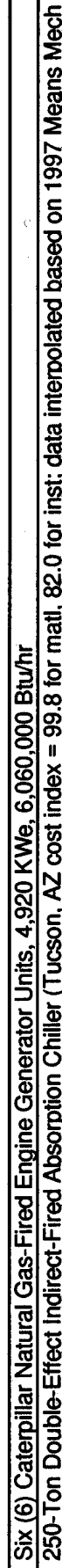 & & 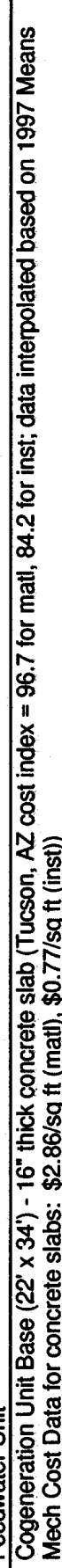 & 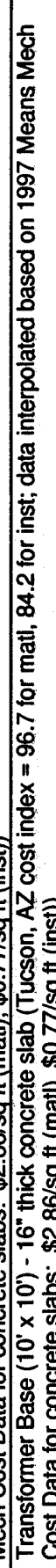 & 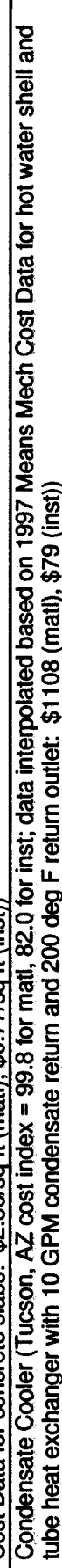 & 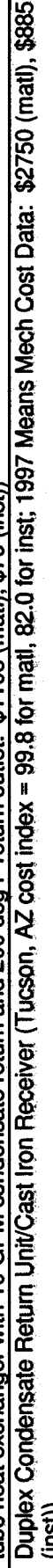 & 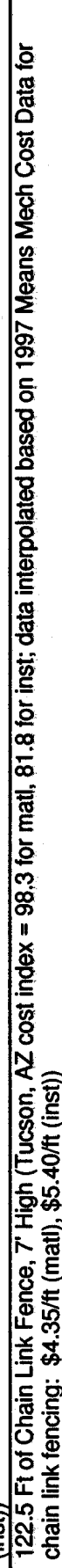 & 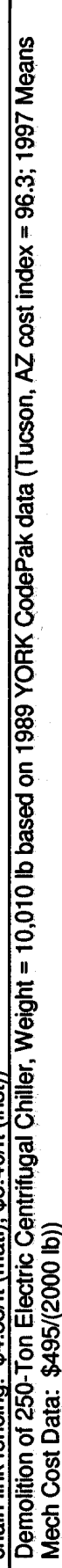 & 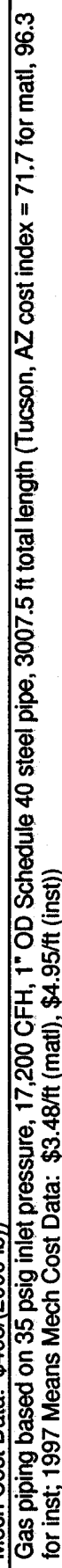 & 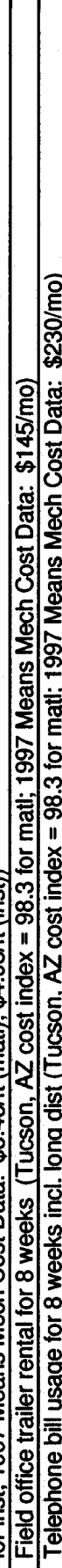 & 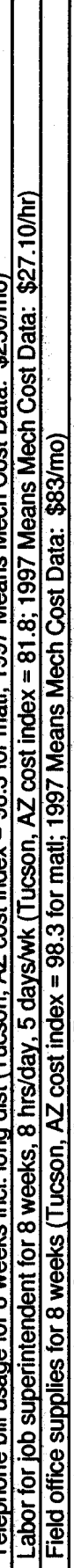 & 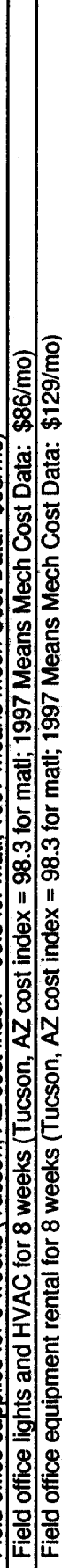 & 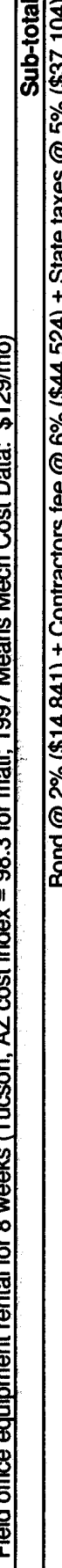 & 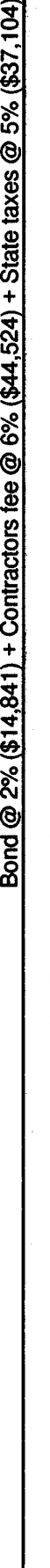 \\
\hline
\end{tabular}




\section{Appendix K: Energy Cost Estimates for Each Option}



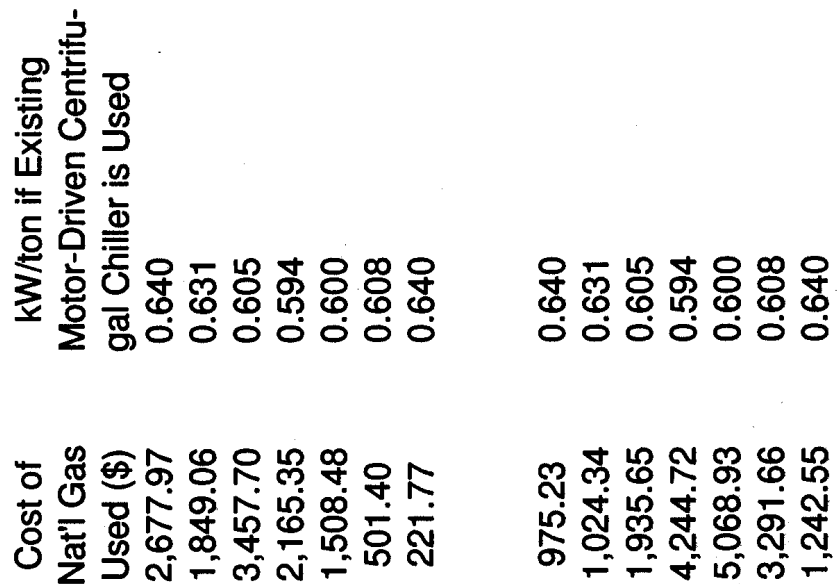

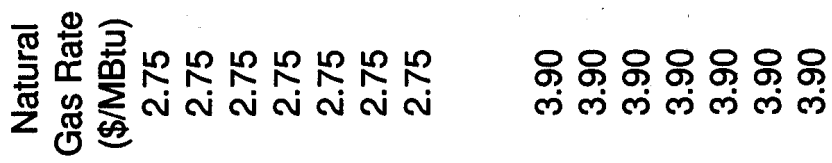

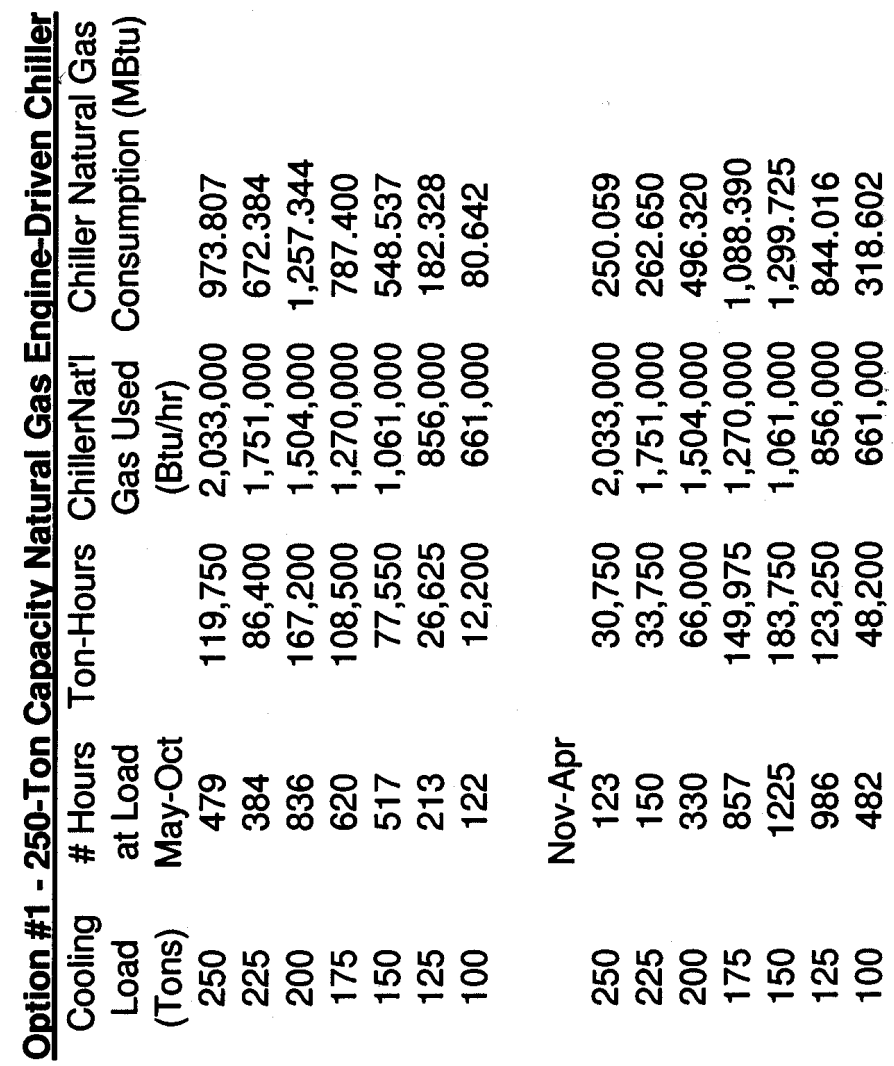




$$
\text { 它 }
$$

Wn!

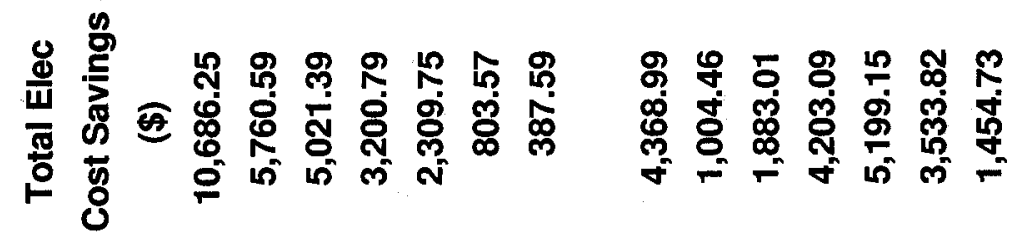

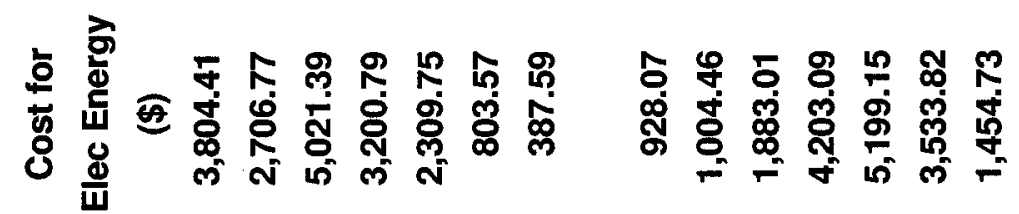

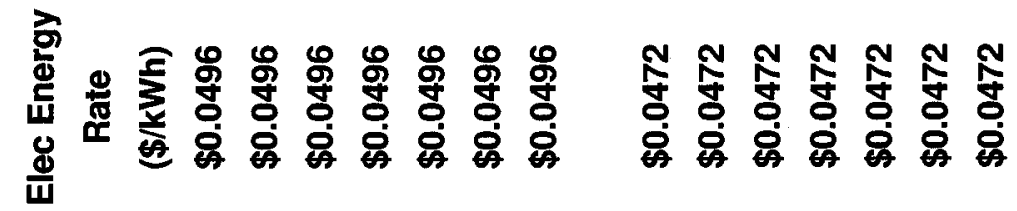

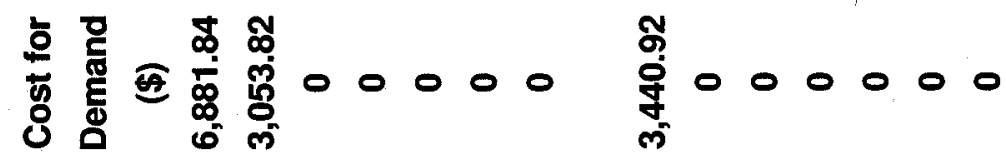

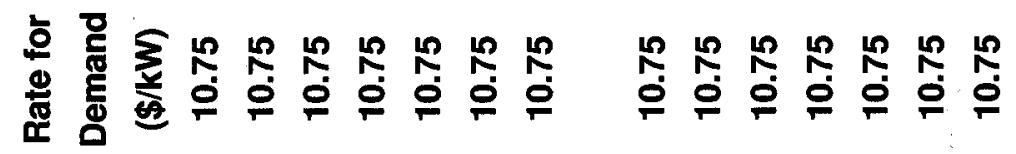

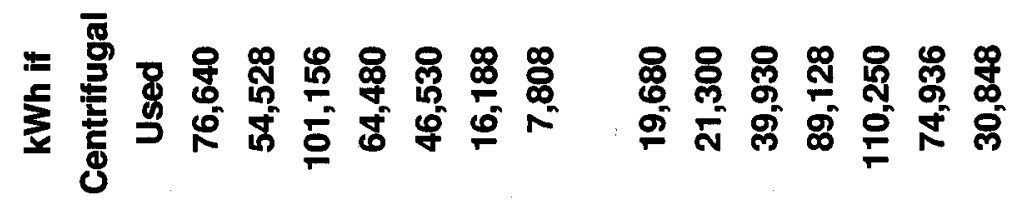

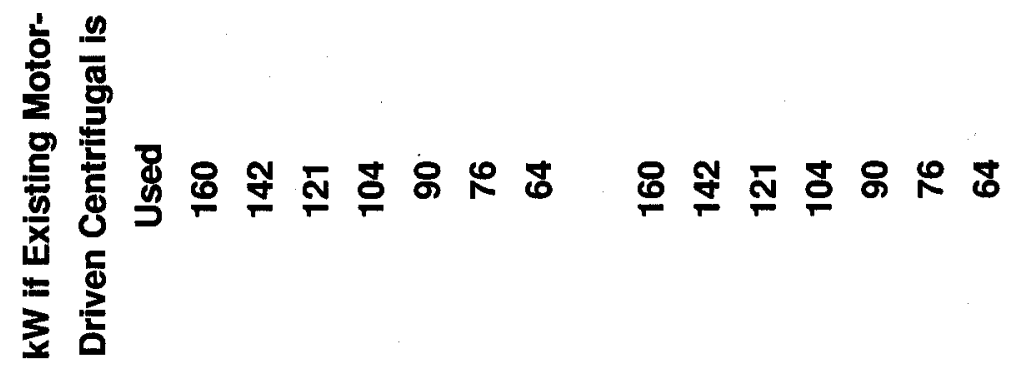



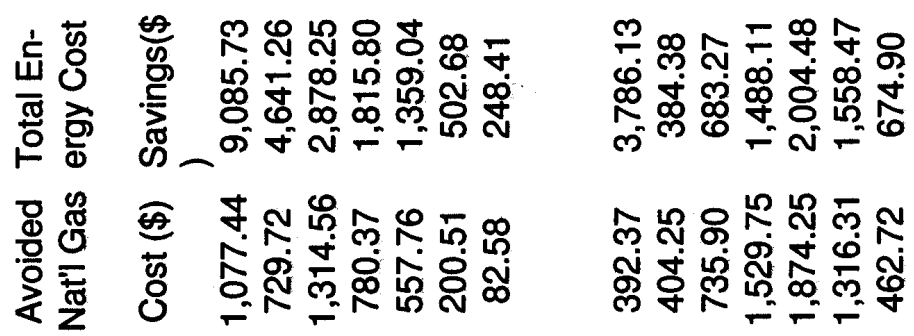

నิ นึ

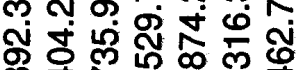

ल द

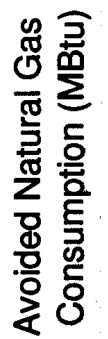

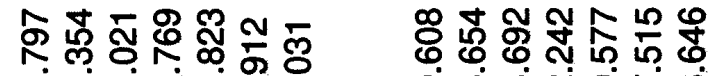

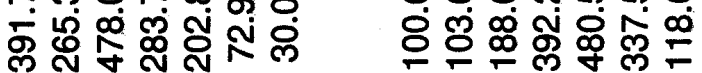

ర్ర

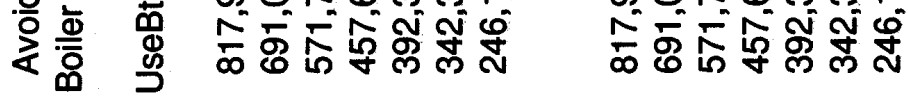

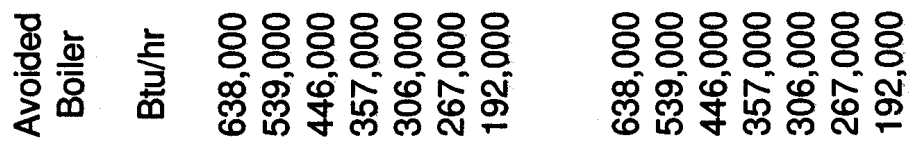

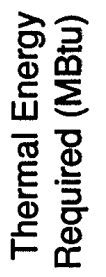

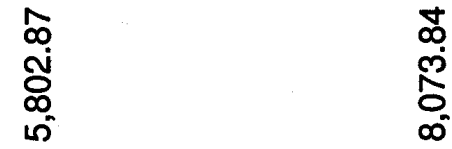

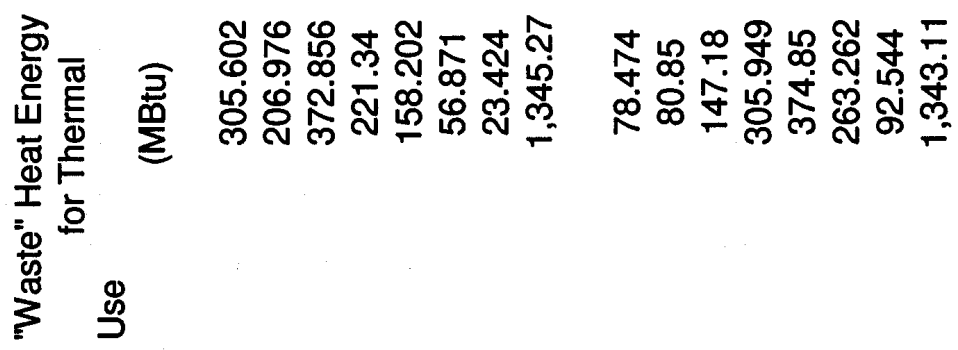



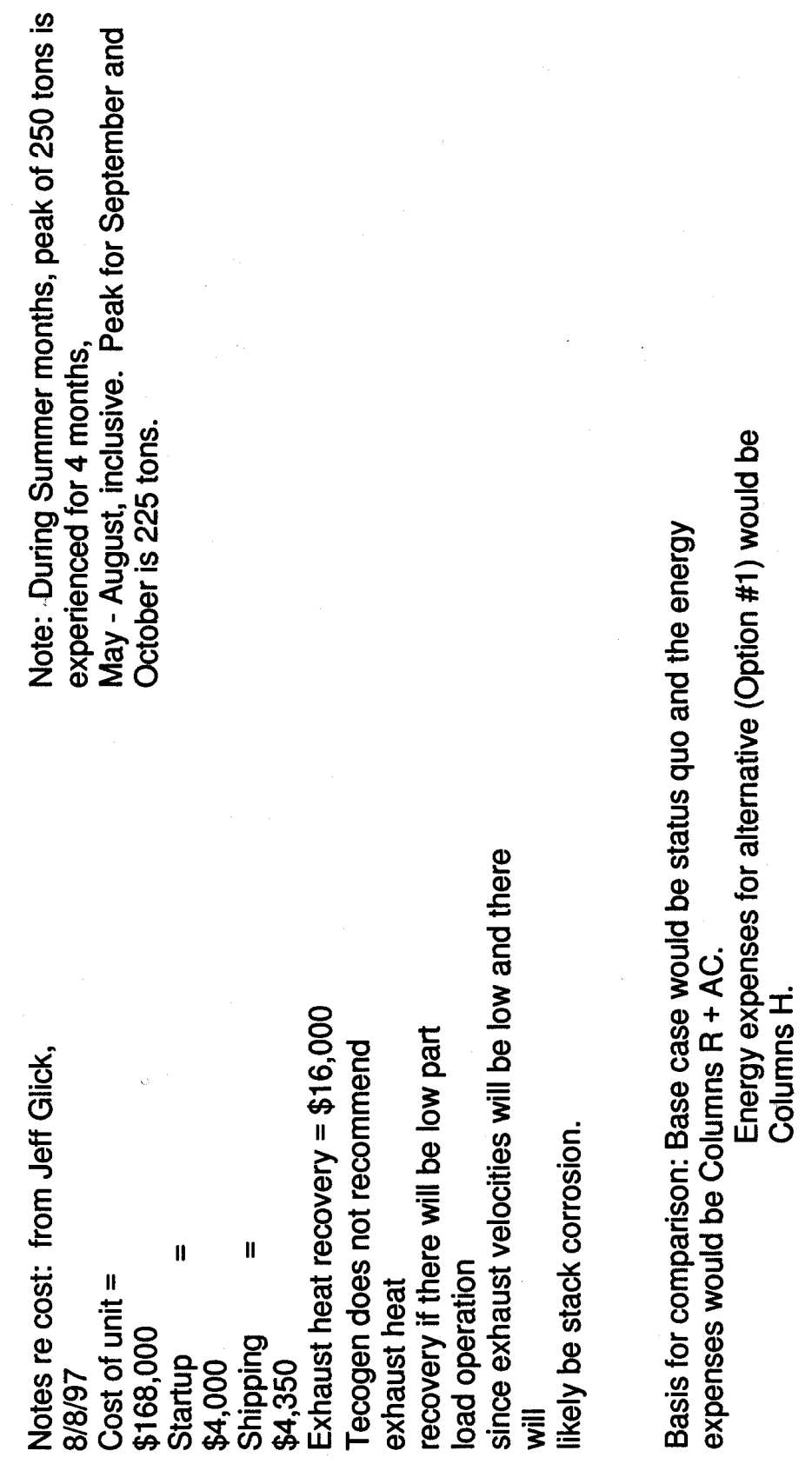


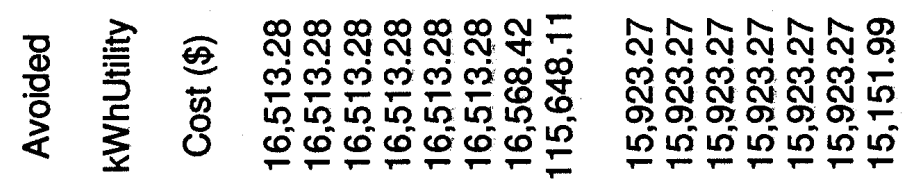

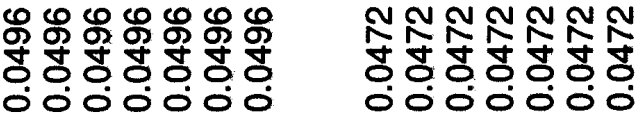

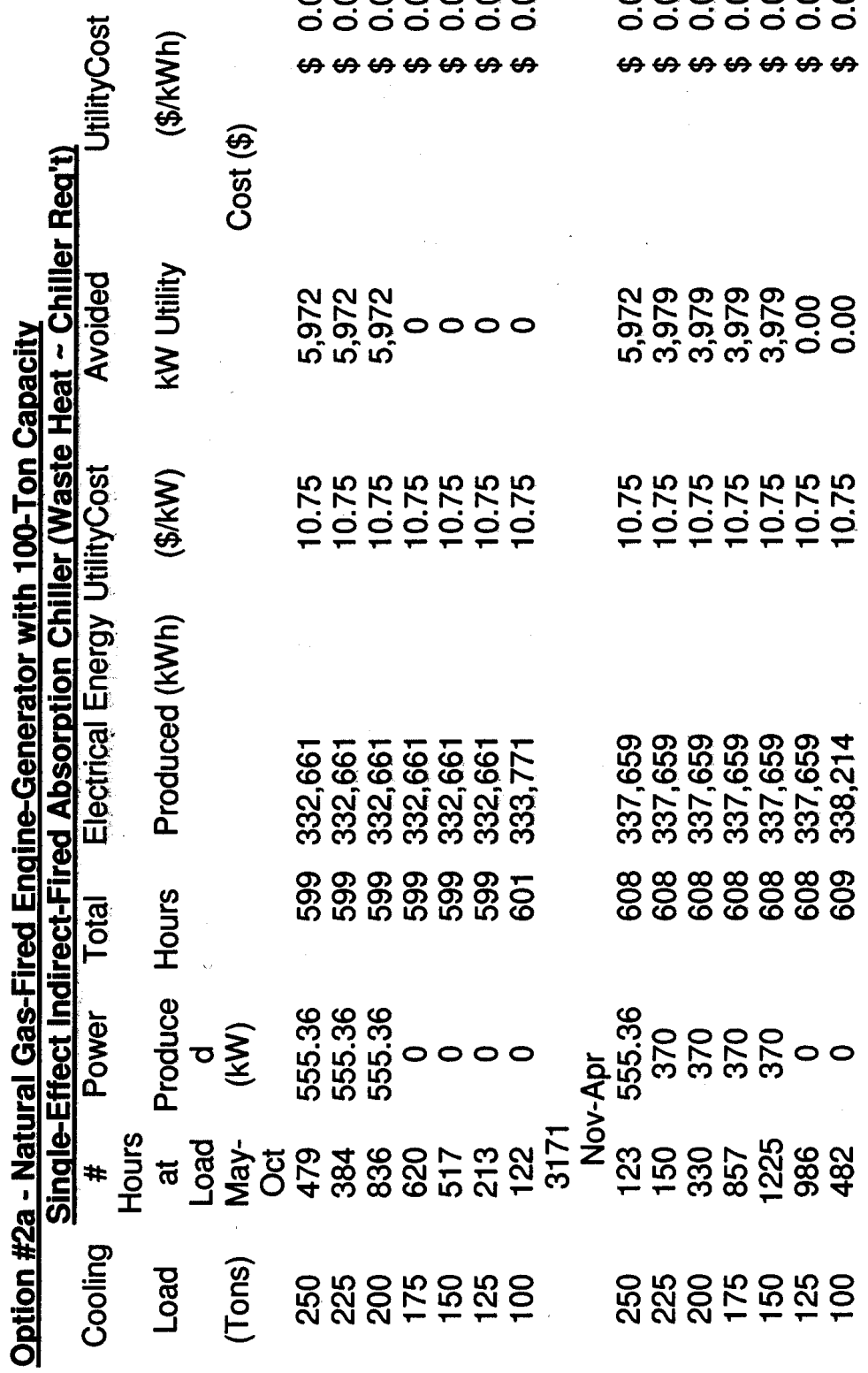




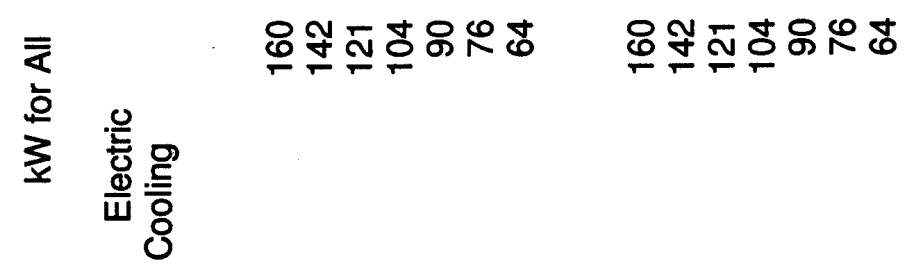

言 道

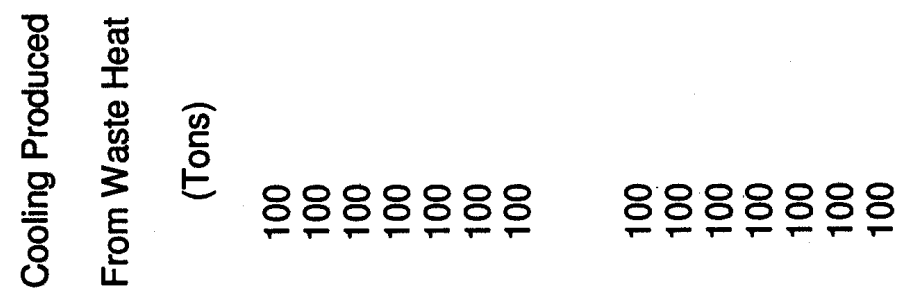

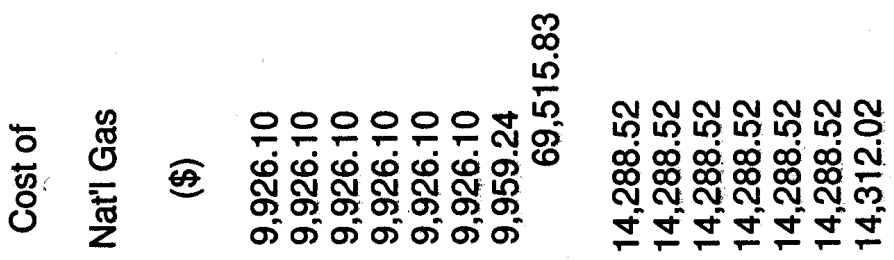

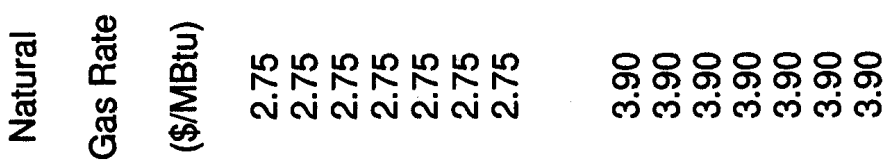
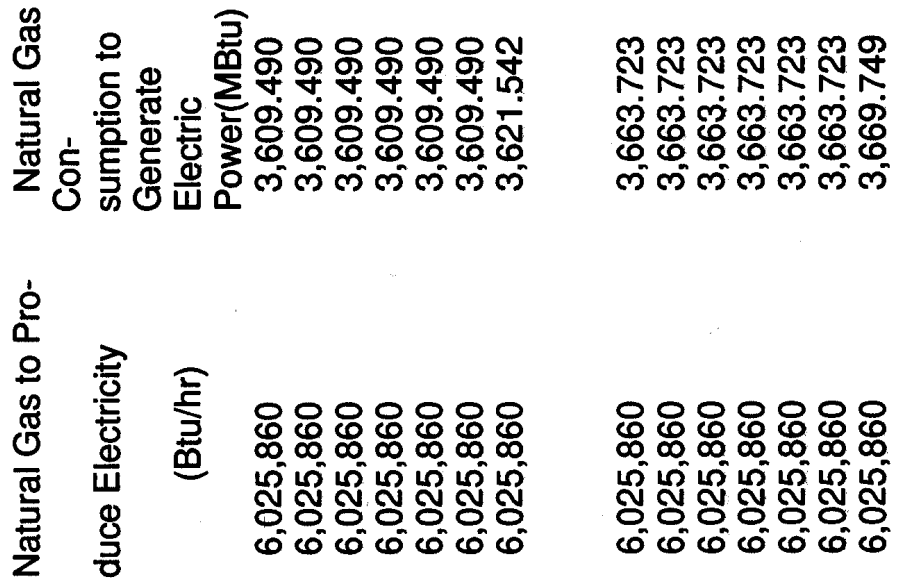


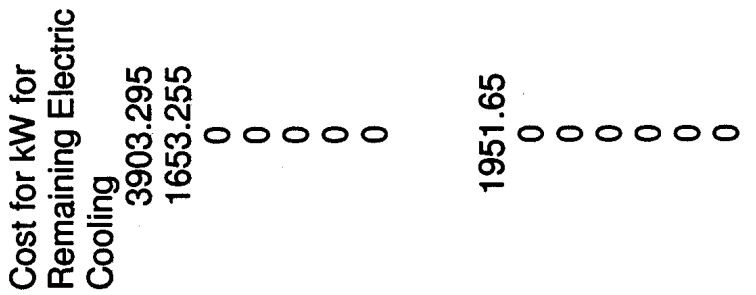

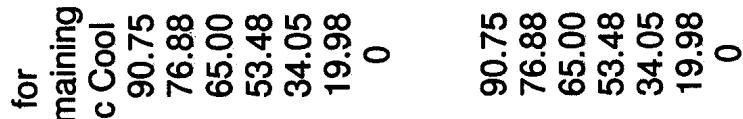
立它思

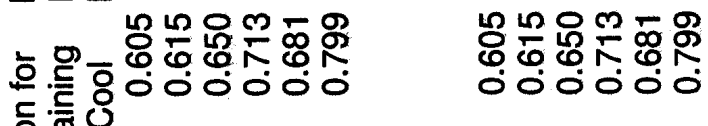

등

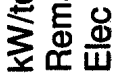

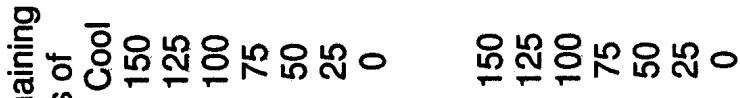
E气 잉

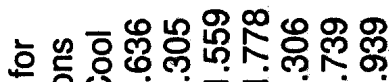

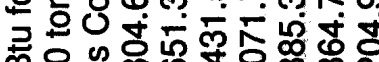

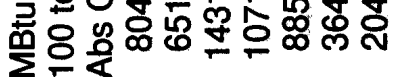

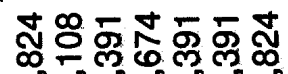

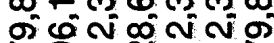
ถ⿻ำ

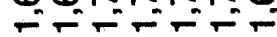

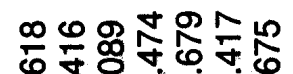

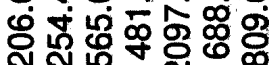
舟

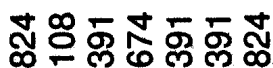
की ㅇํㅇ츛ำ

훙ㅇํㅇ

홍으운

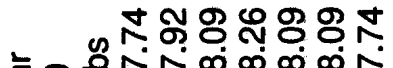 \\ \#흐융}

ㅊํㅇ유융ํㅠ

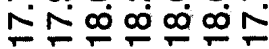

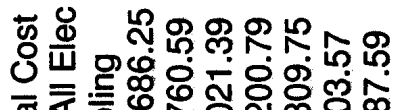

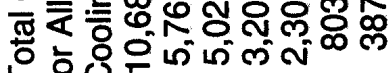

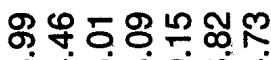

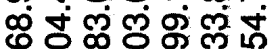

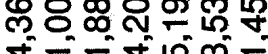

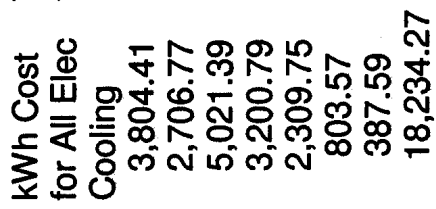

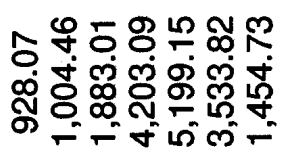

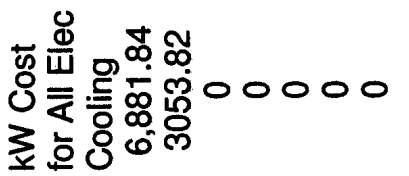
๙ั 


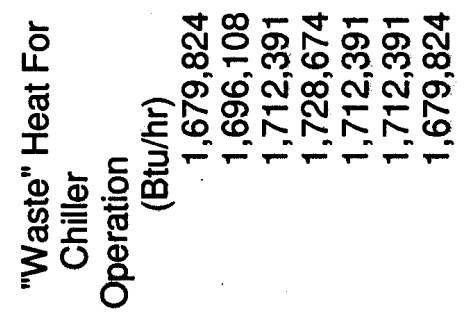

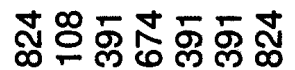

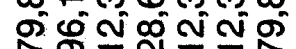
0조중

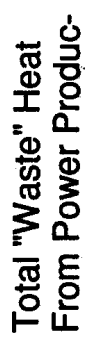



융ㅇㅇㅇㅇㅇㅇㅛ 훙형ㅎㅎㅇㅎㅇㅎㅇㅇ 형형ㅎㅇㅎㅎㅇㅎㅕ

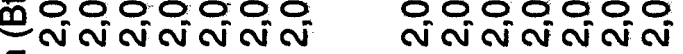

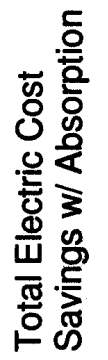

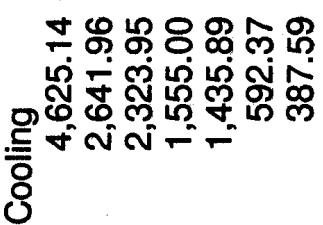

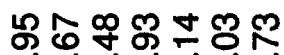

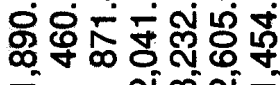
ठํ.

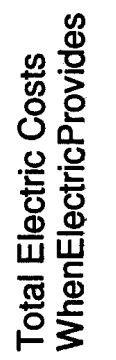

응

D=

宓

ॠ

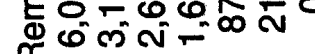

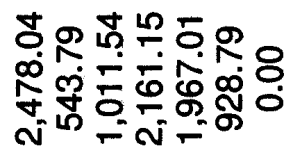

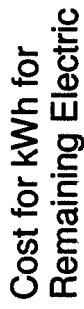

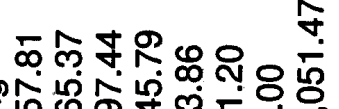

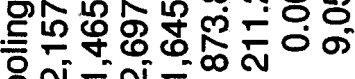

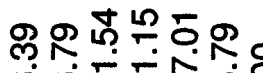

ஸิ่ $\mathcal{O}^{2}-\mathrm{n}-\infty \mathrm{n}$

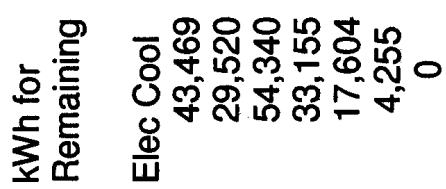

ชิธ - no Fテลัง ษ 


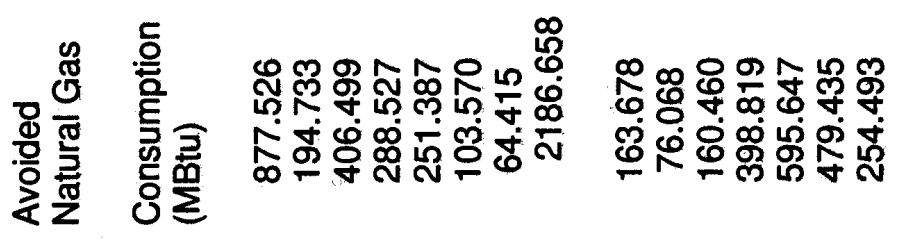

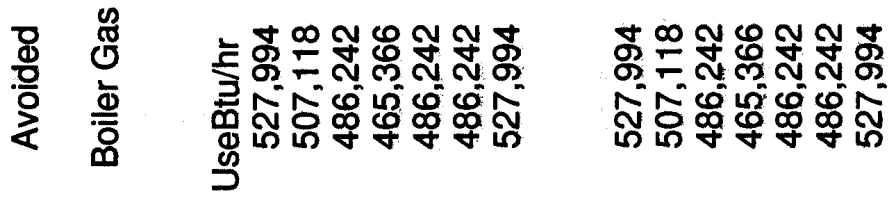

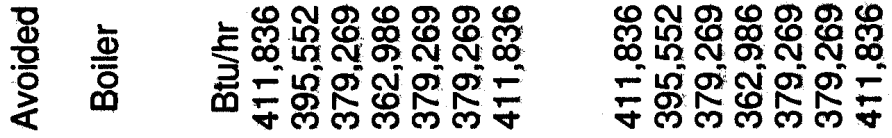

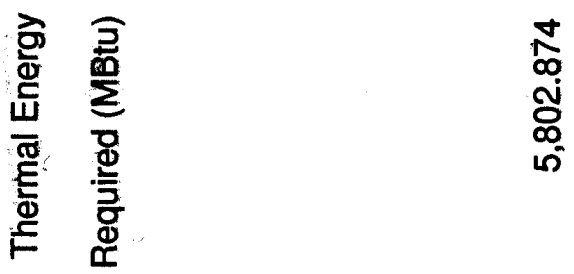

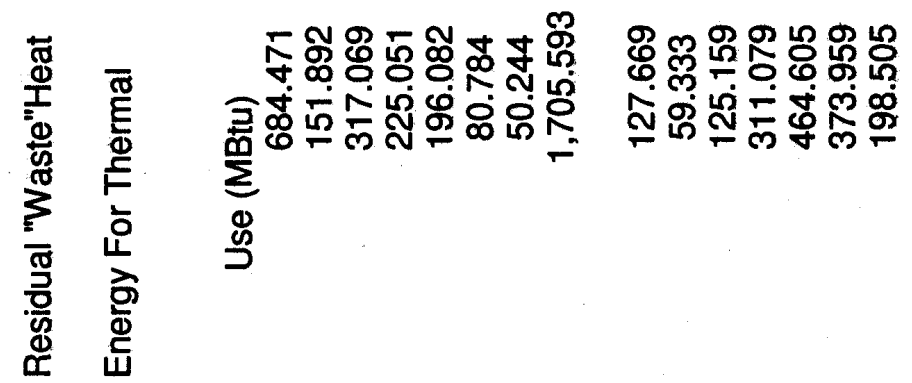

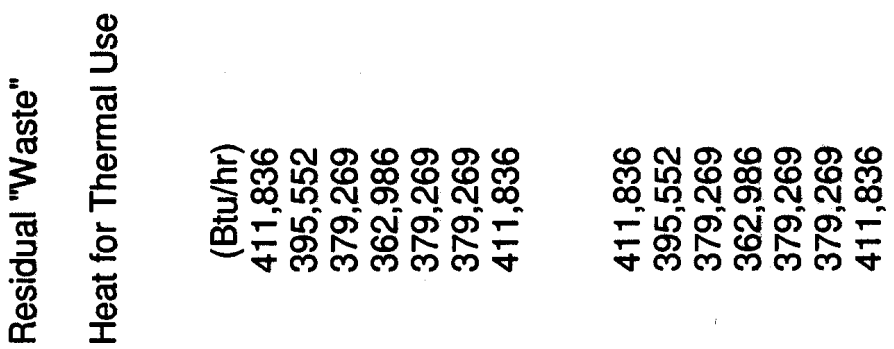




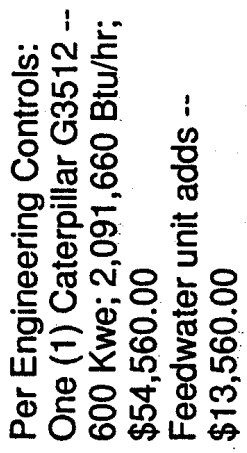

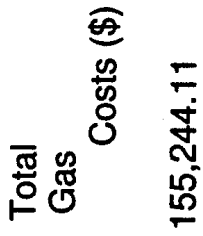

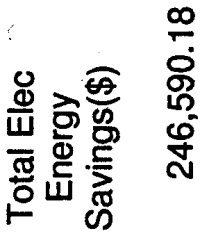

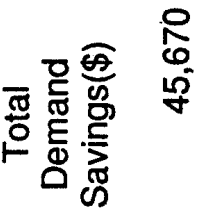

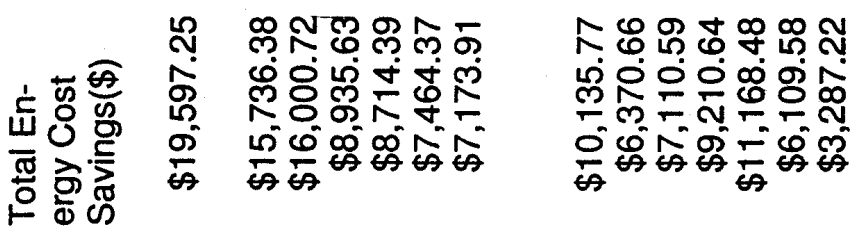

\begin{tabular}{l}
$\dot{0}$ \\
+ \\
$N$ \\
+ \\
$\dot{x}$ \\
$\stackrel{0}{E}$ \\
$\stackrel{E}{5}$ \\
$\dot{0}$ \\
\hline
\end{tabular}

\&

읗

के

$\Phi+\Phi$

동 뚱

\%

ㅎํㅇㅇ

용

흑응

을

ส

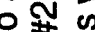

음

을응

恶응

잉

윽 究造

离 흥

of

\&

岇 ఖ

它

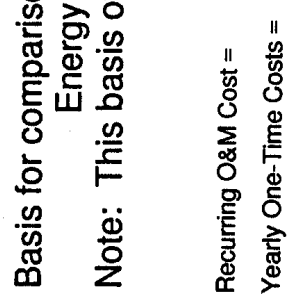

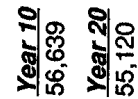

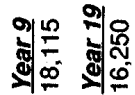

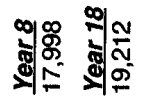

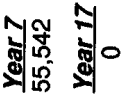

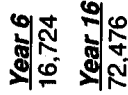

ํํำ

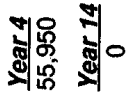

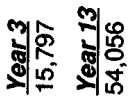

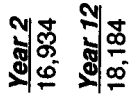

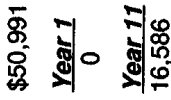




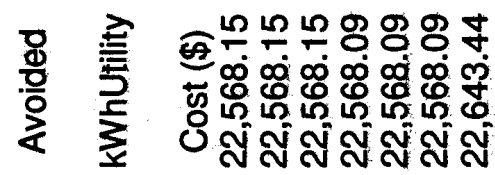

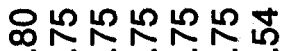

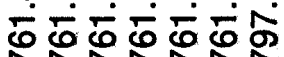

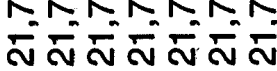

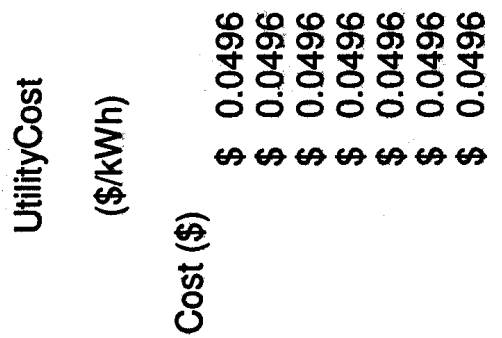
NNN吕罗

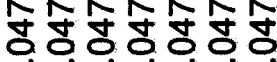

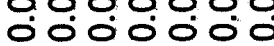

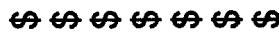

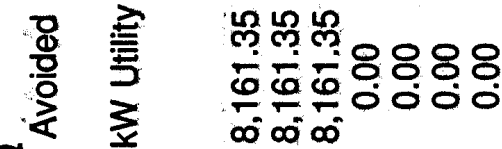

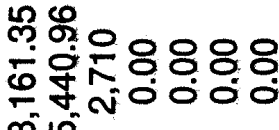

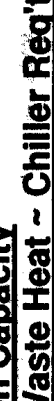

웡

乌 응ㅇㅇㅇㅇㅇㅇㅇㅇㅇㅇ

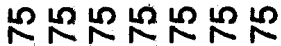
으으으으으으은

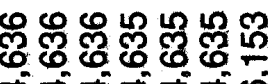

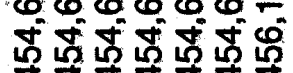

됴요 ช + চீர்

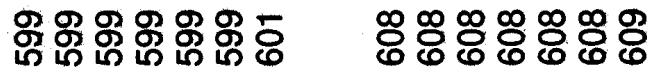

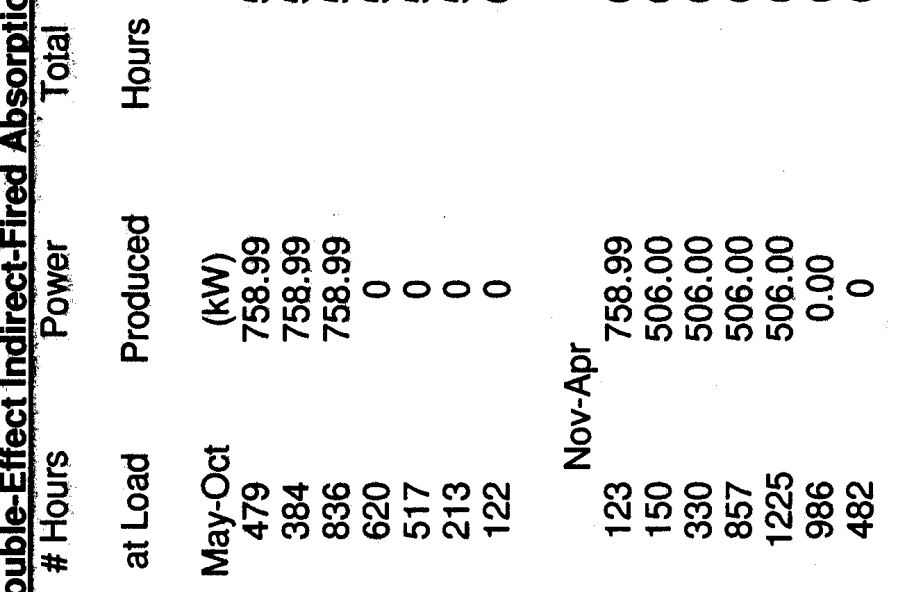

응 ఫ 


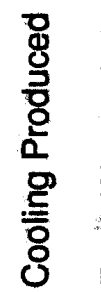

홍

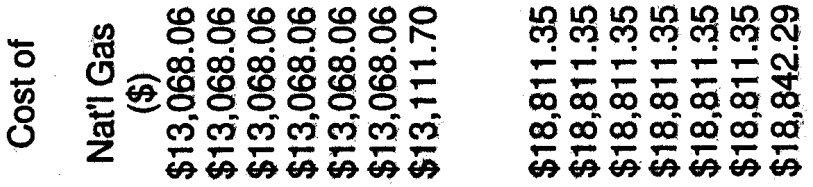

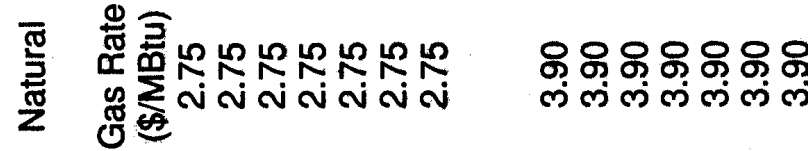

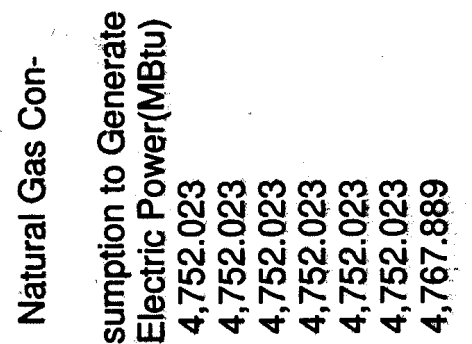

พิ พิ พิ พิ

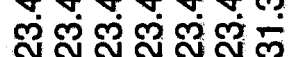

న

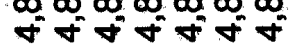
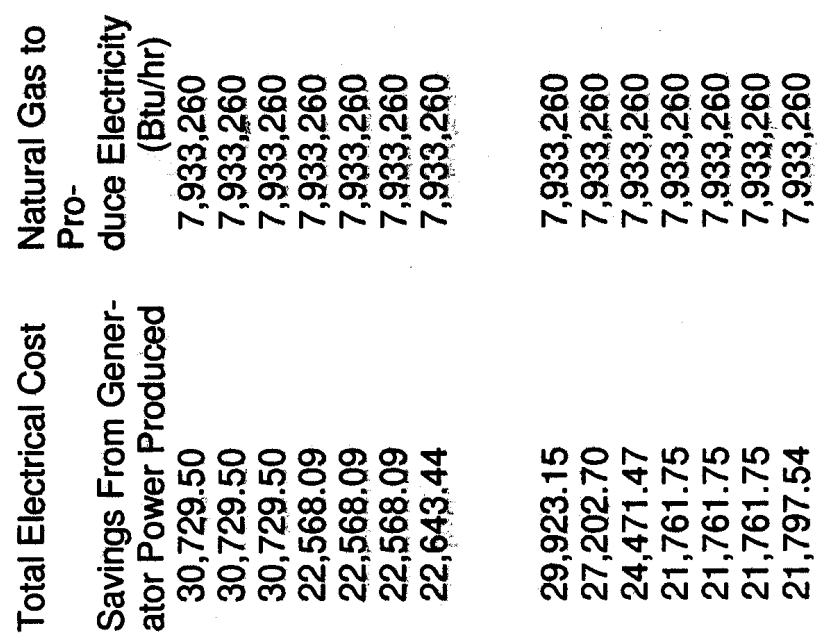

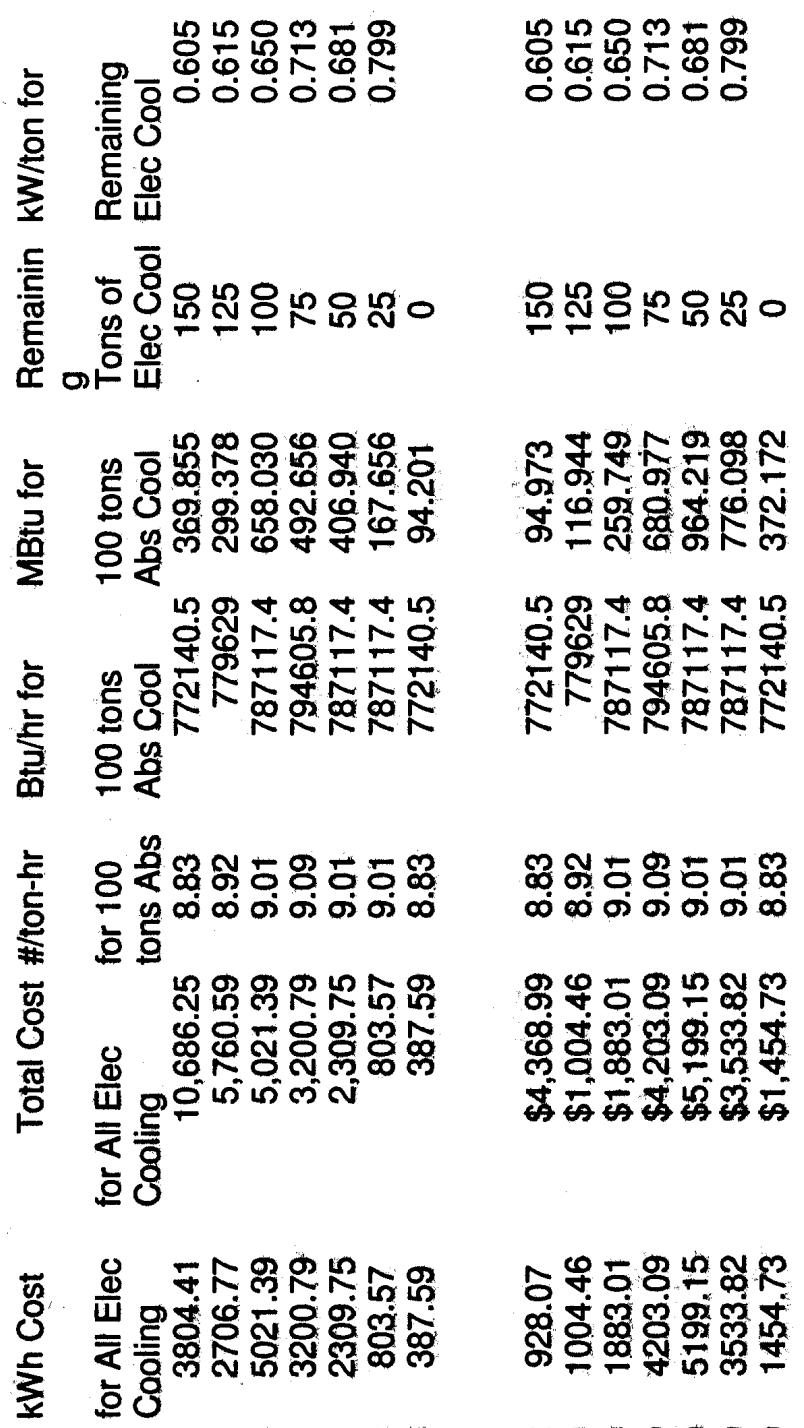
๘

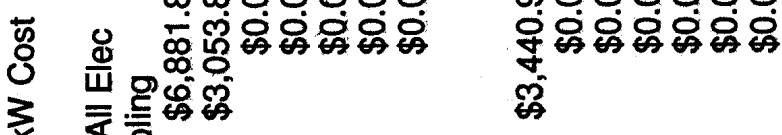
立辰語心

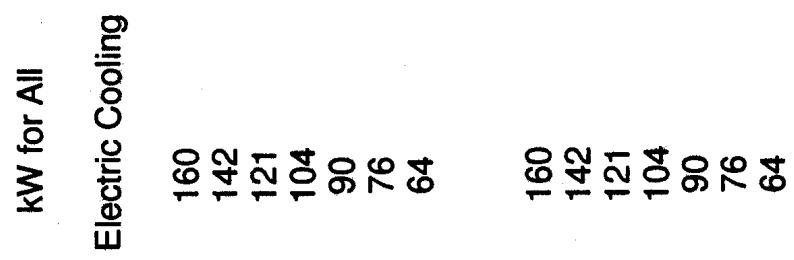

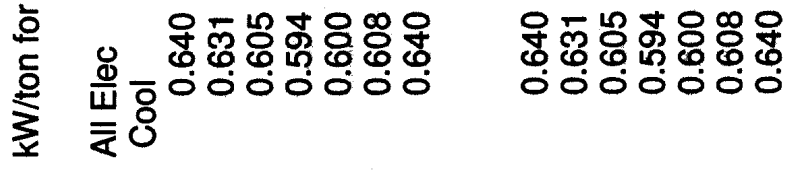



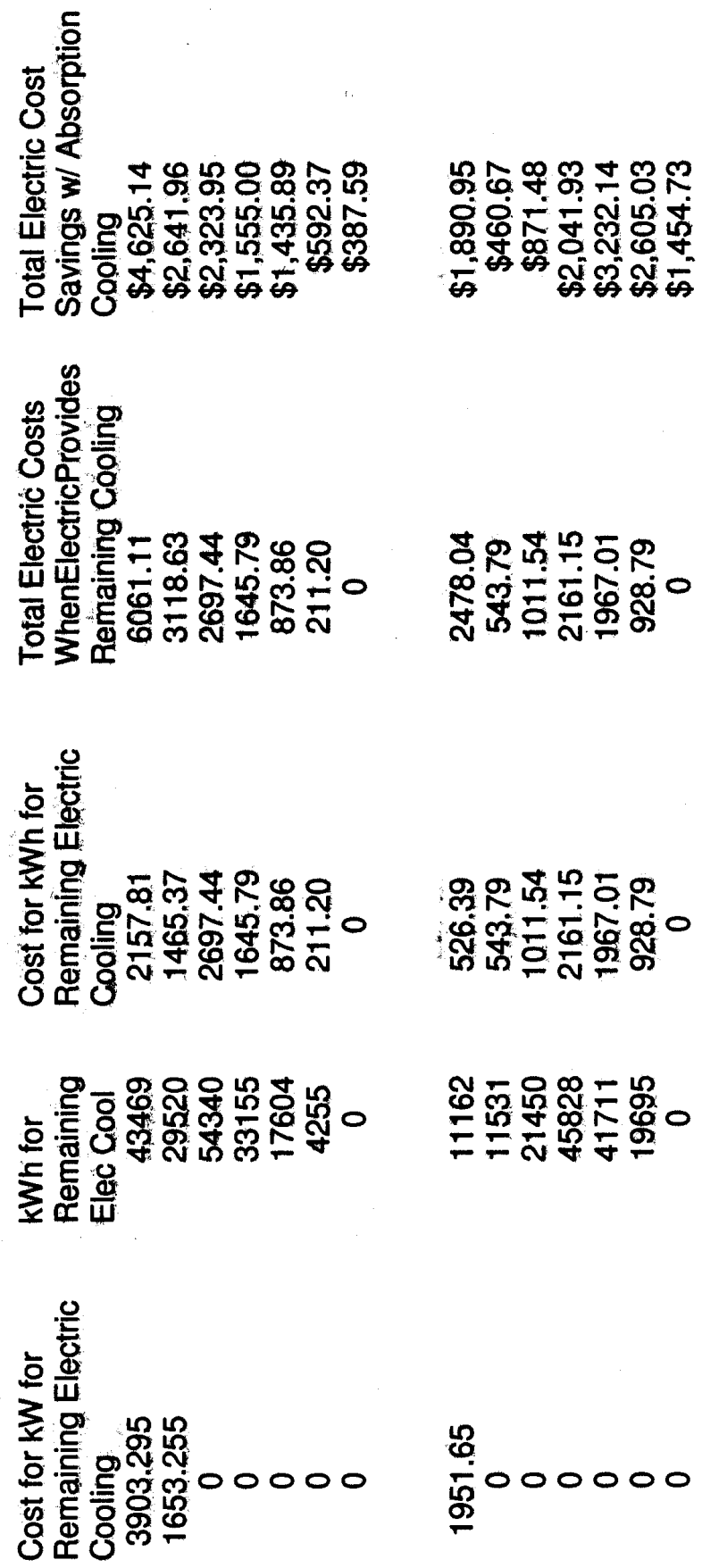

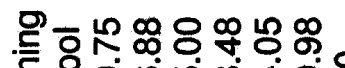

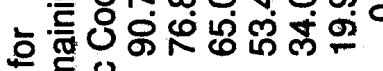
文高畨

ํㅛㅇㅇㅇㅇㅇㅇ

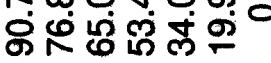




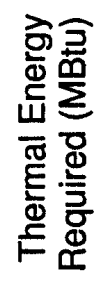

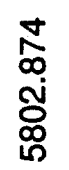

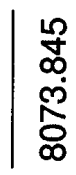
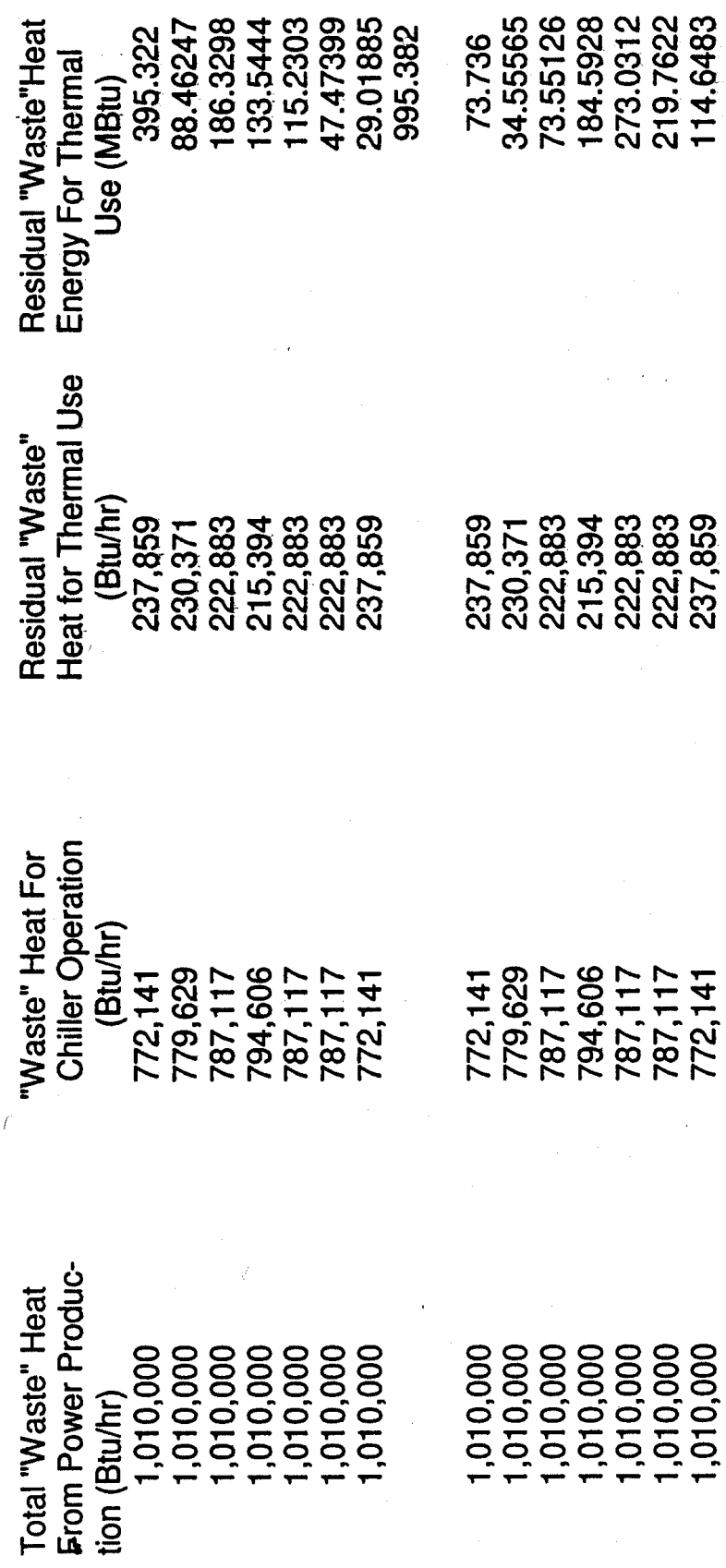

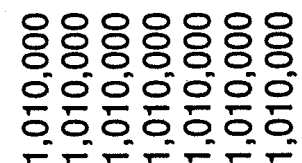

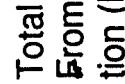




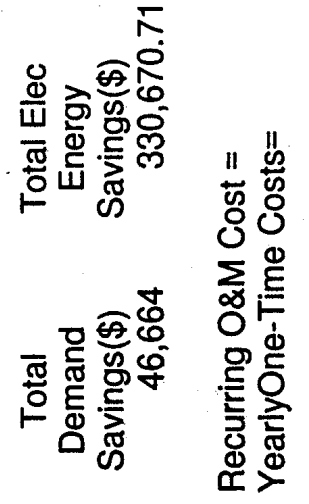

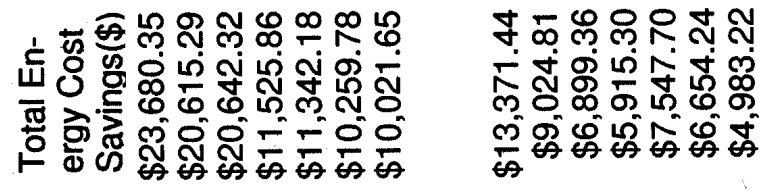

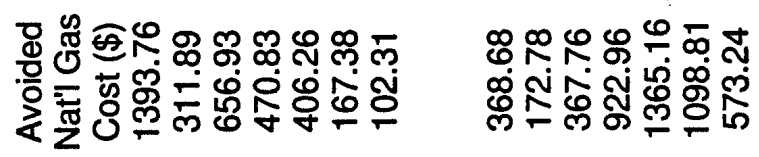

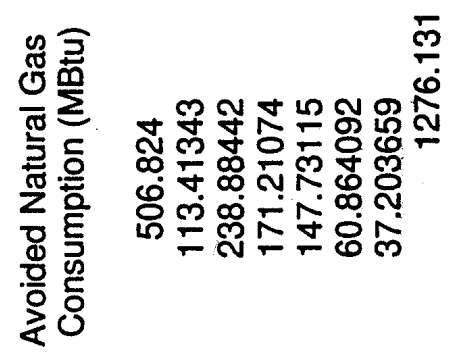

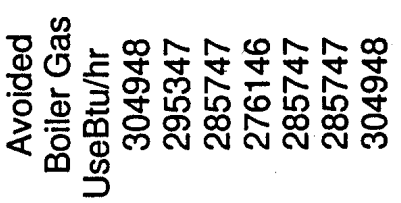

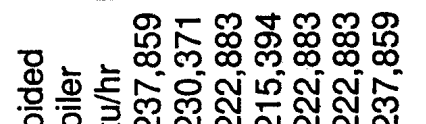

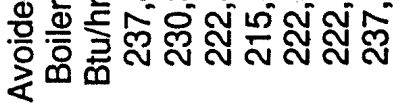

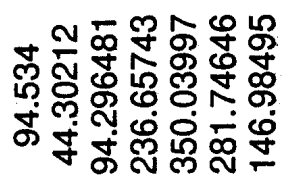

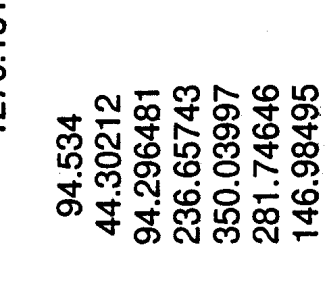

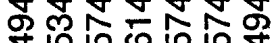

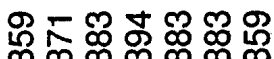
ల్ $\infty$ ల్ $\infty \infty$

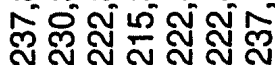

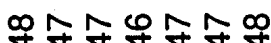
㧀㣽哭品品 


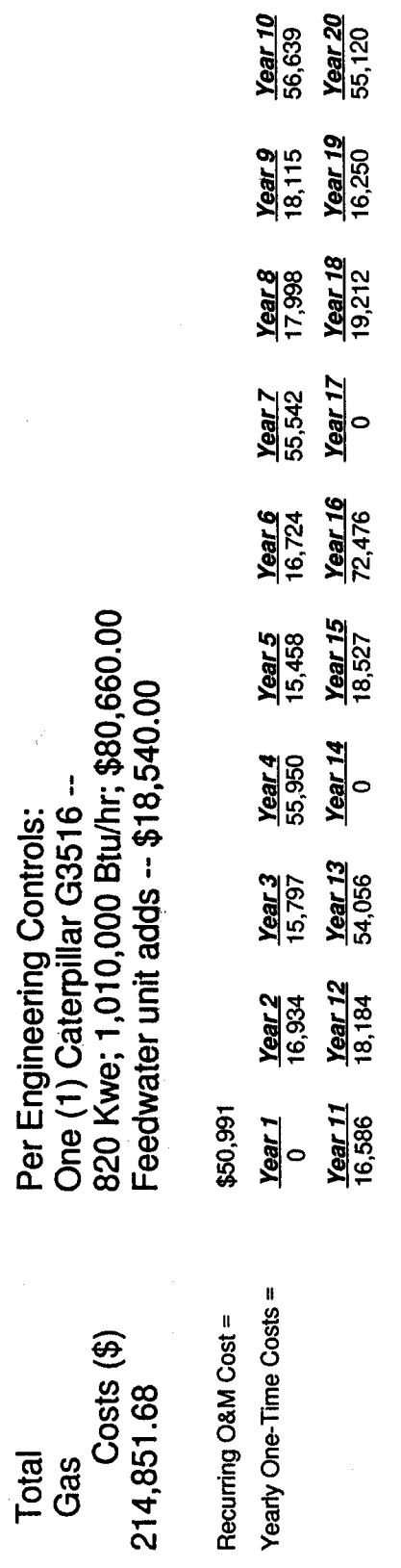




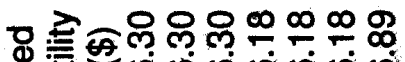
क 능 ल ल ल ल

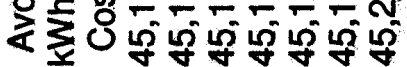
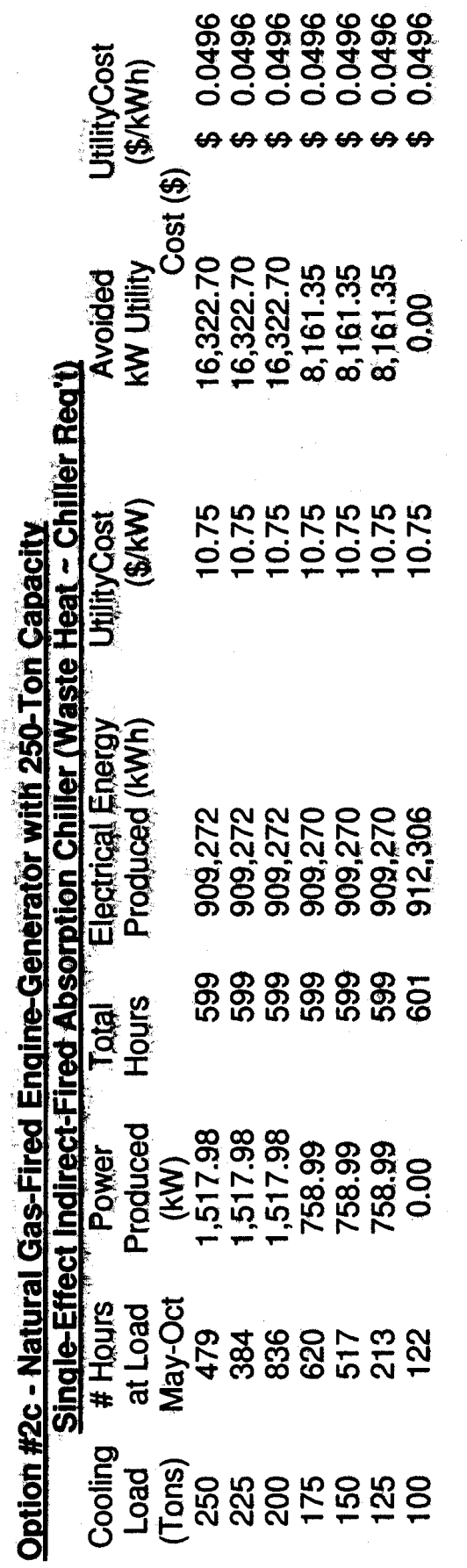

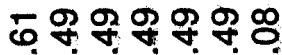
ตุ่มี่

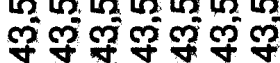

ํํํํํำ ฟิ่ ชิ่ ชี่ ชั ले ळूळ 응ㅇㅇㅇㅇㅇㅇ

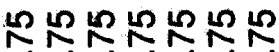
으으으으으으웅

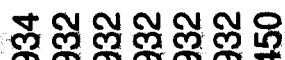
\%

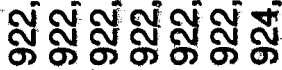

웅 웅융융용용

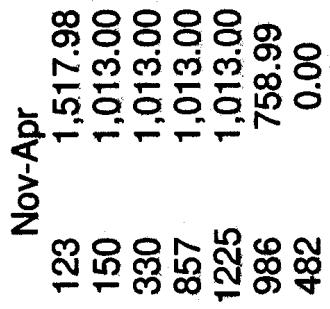

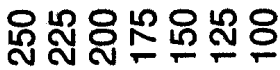



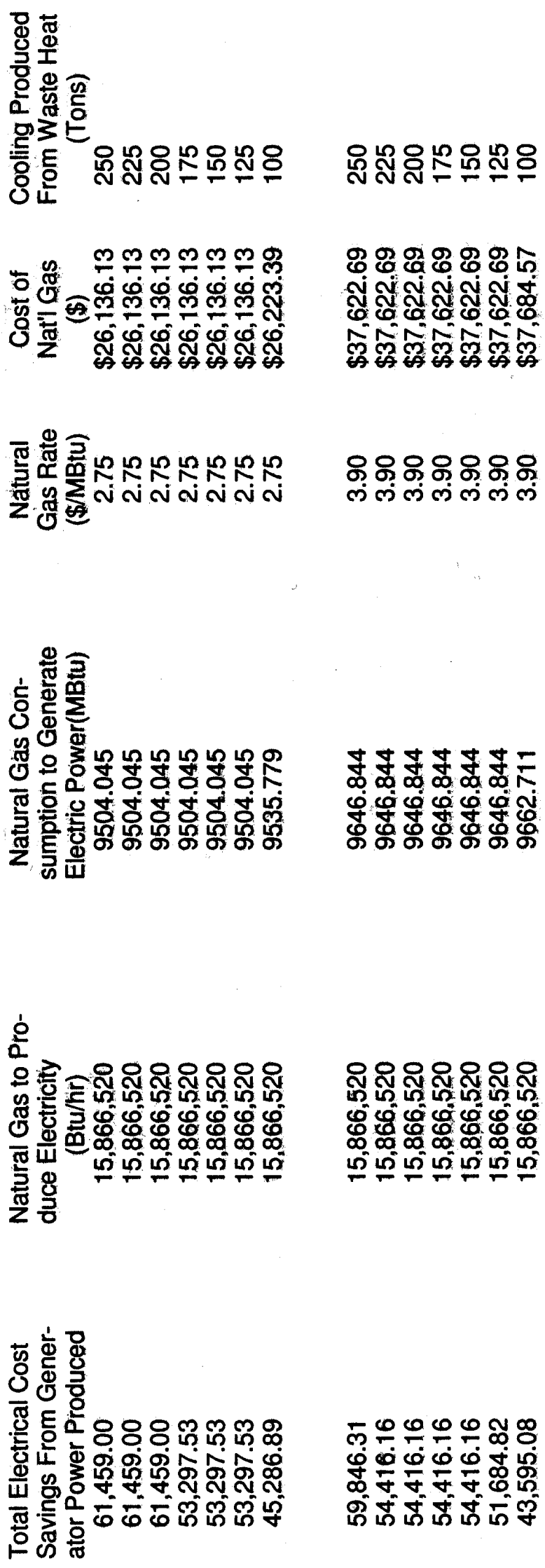


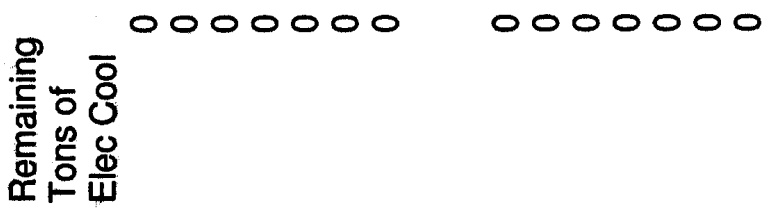

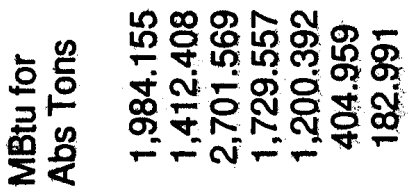

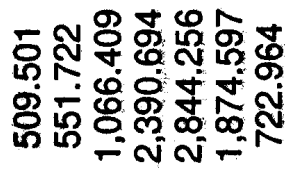

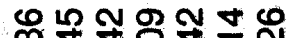

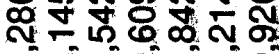

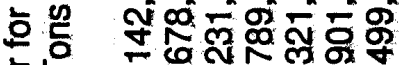

ㄴำํํㅇํำ

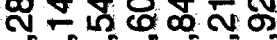

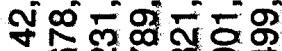

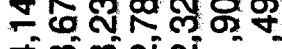

ㅇำ

空 $\frac{0}{\alpha}$

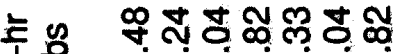

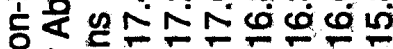

× लंख⿰ - -

\#产응

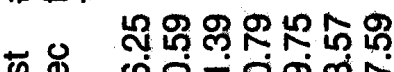

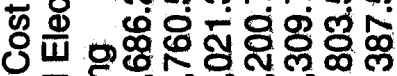

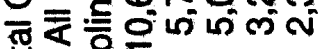

흔흥ㅇㅇ

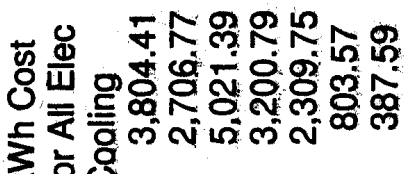

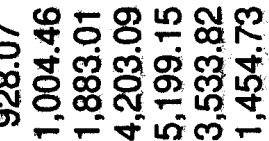

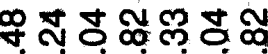

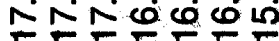

영

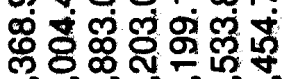

品-

立。ర

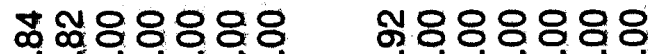

茯

立云言灾息

홍ํㅇ

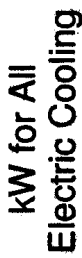

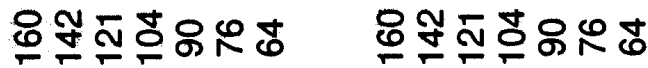

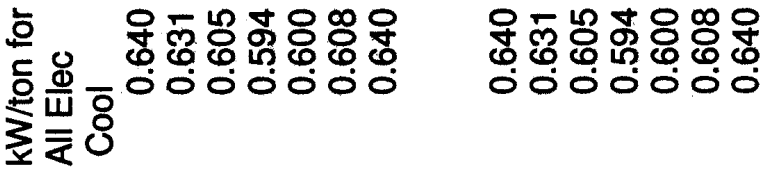



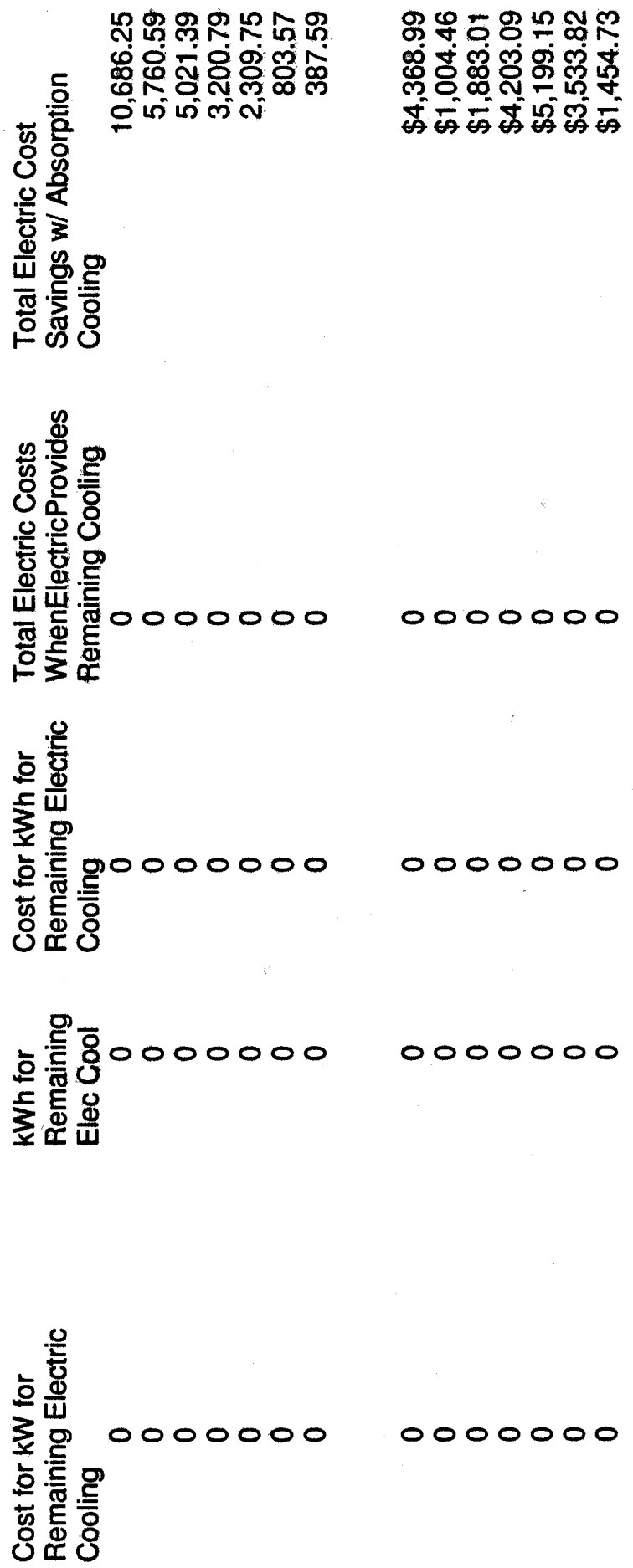

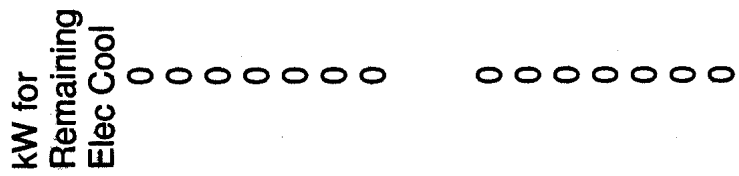

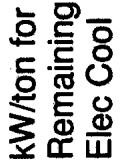




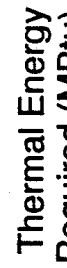

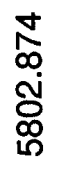

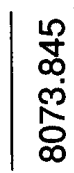
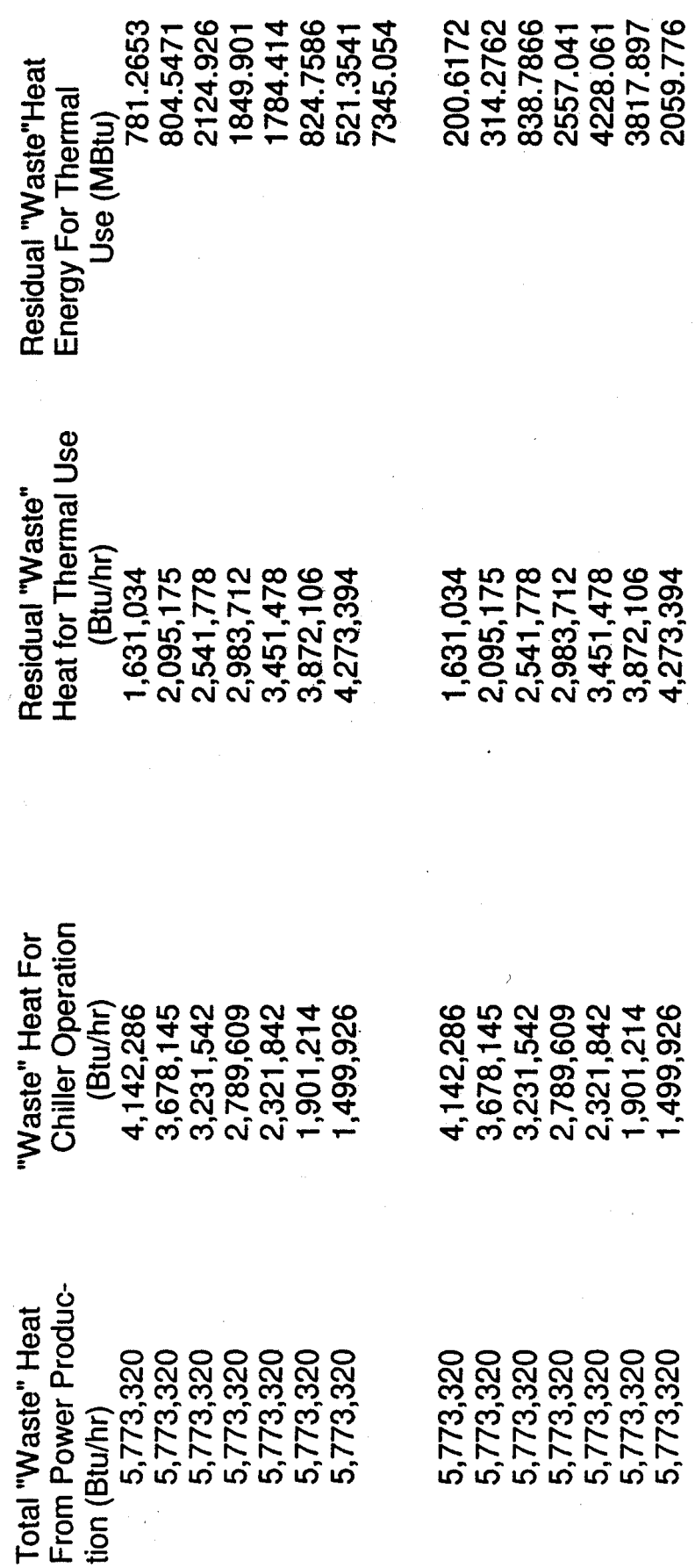

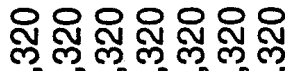
ल लंल ल RANANR जी 흉흔든 

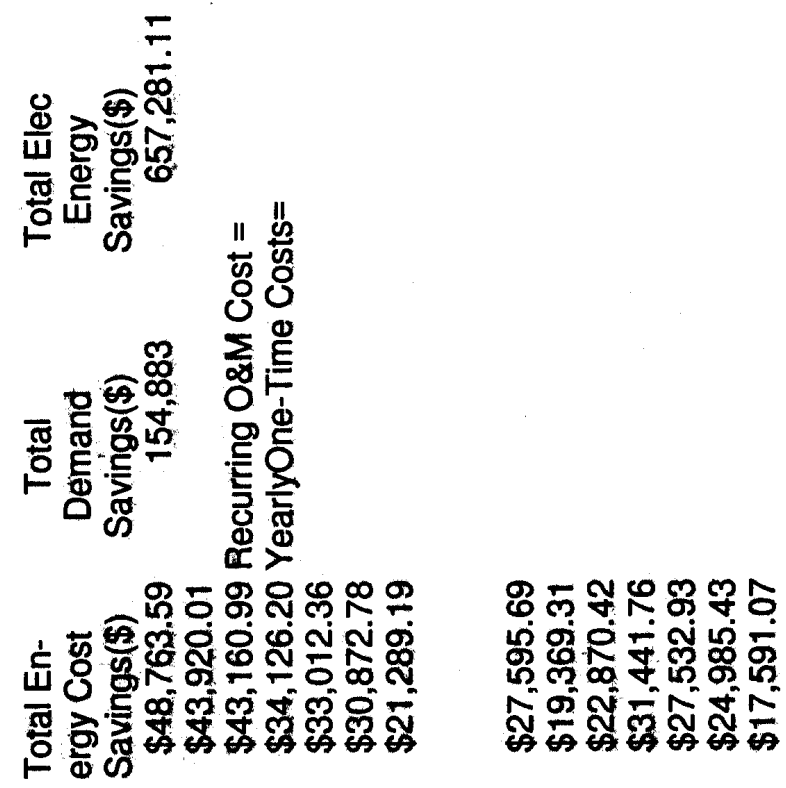

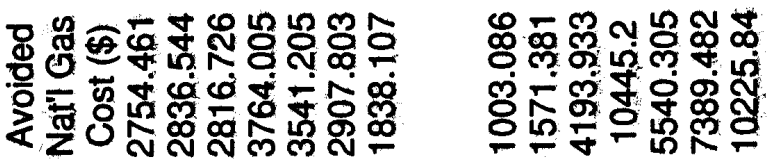

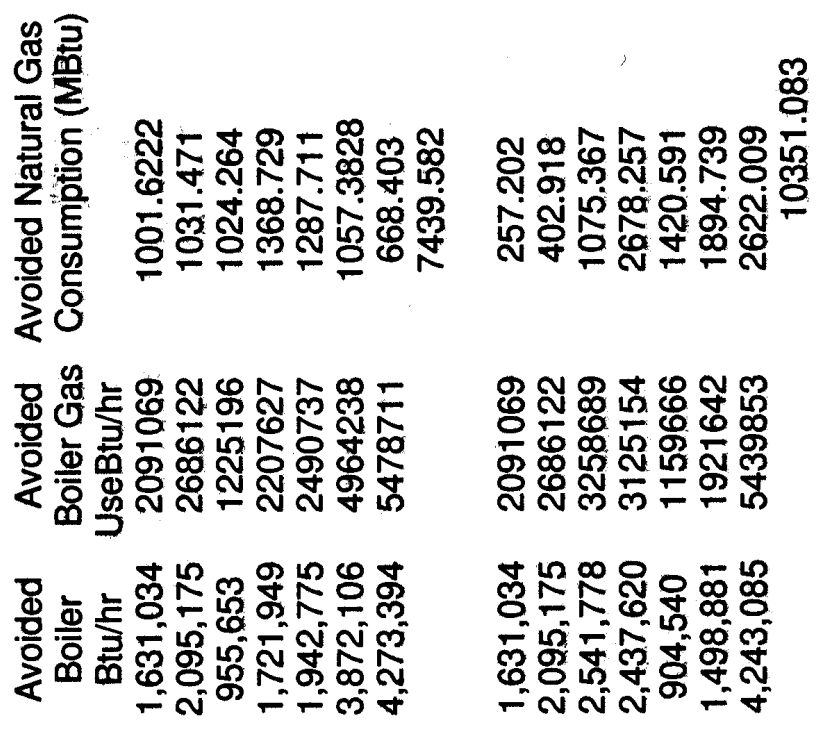




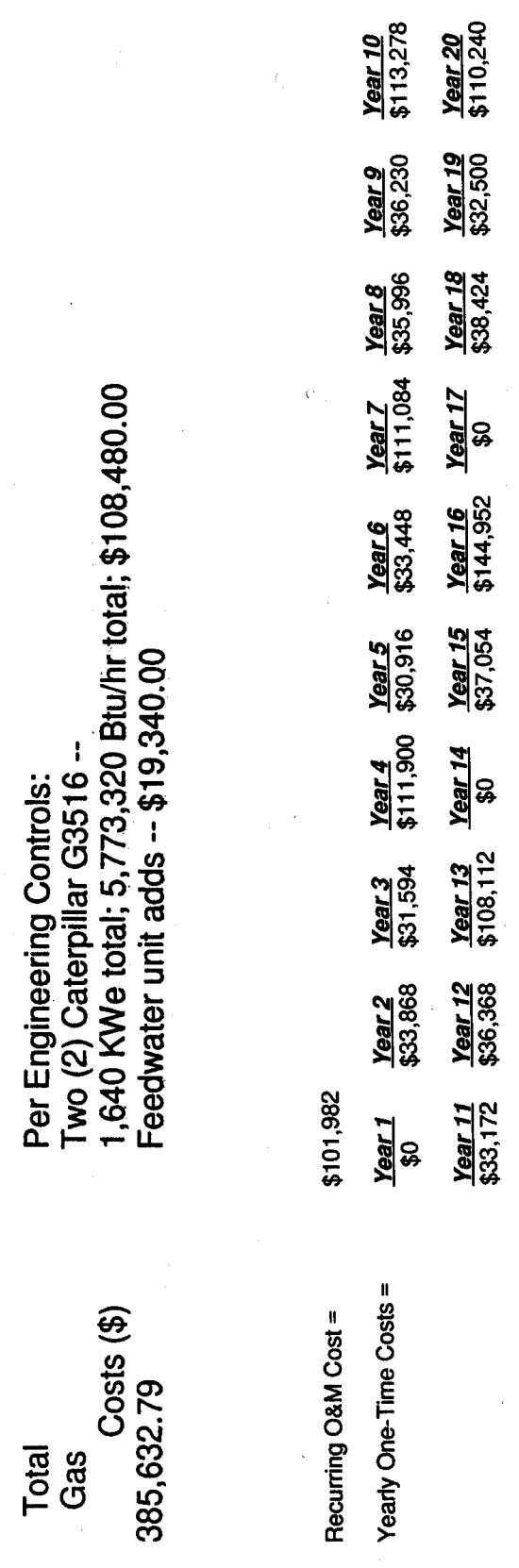




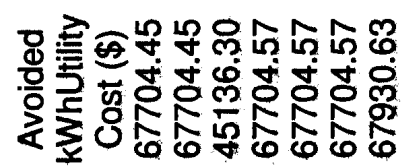
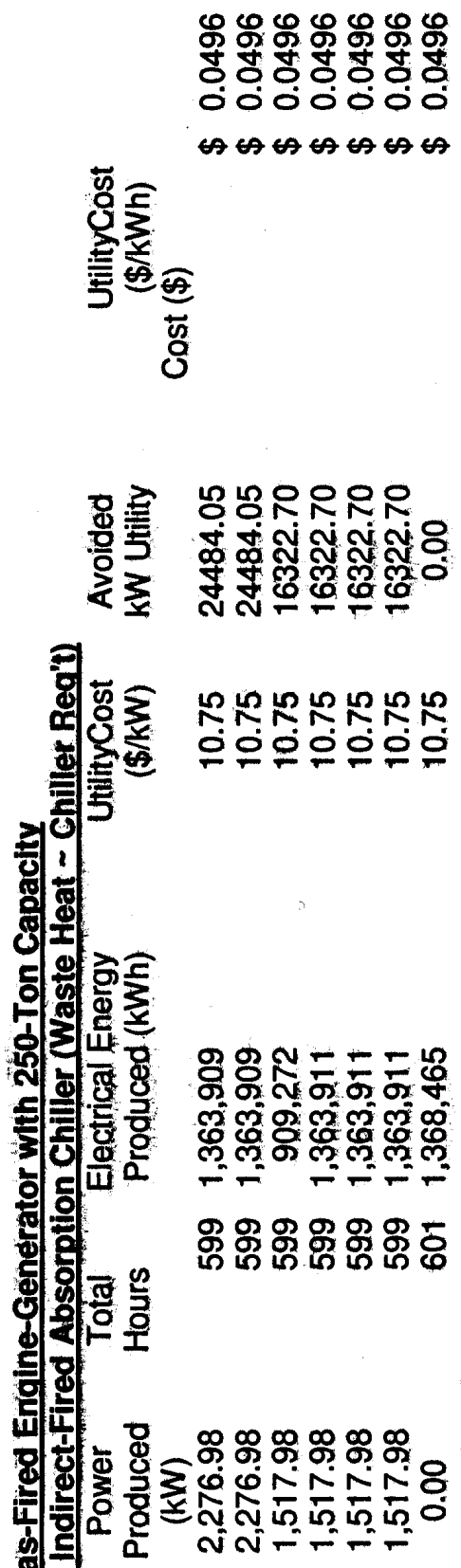

थ0

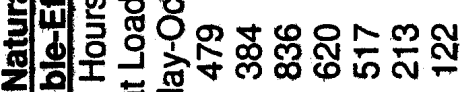

웡ํํ

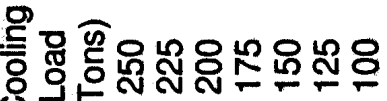

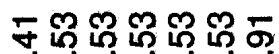
政

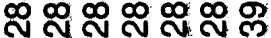

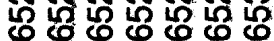

NNNNNNN

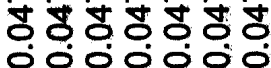

ब大

ㄴํำชูสชิำ में लं ษ 눙유

ص 으응ㅇㅇㅇㅇㅇㅇㅇㅇ

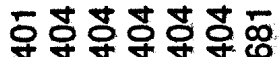
- $\forall-6$

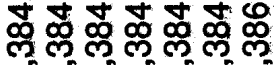
ल-

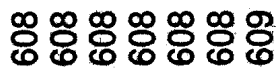

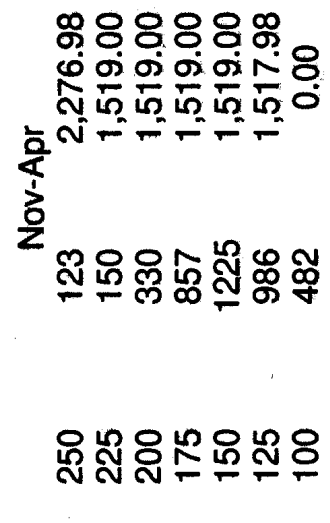


马

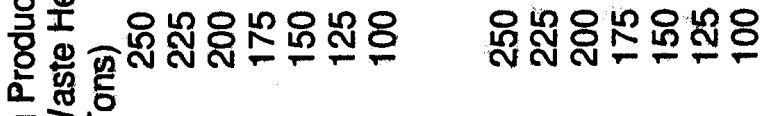

은

응 톤

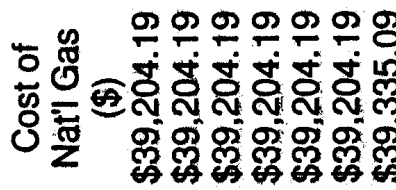

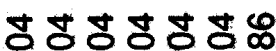

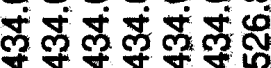

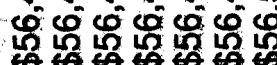

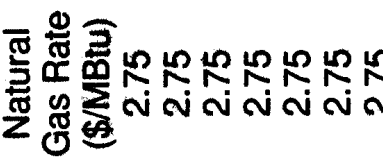

요요요요용

लंलंल लंल

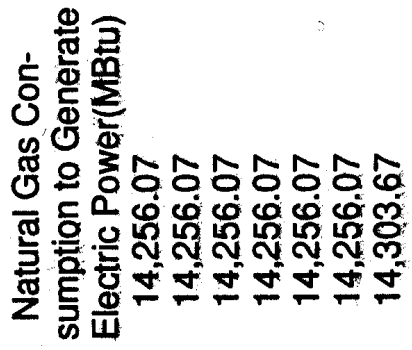

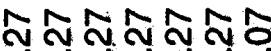
우옹영

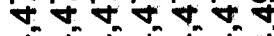
ザずずずす

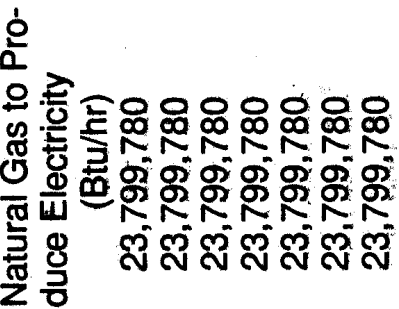

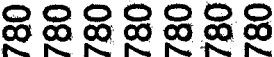
\%ं $\sigma^{\circ} 0^{\circ}$ कर

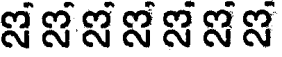

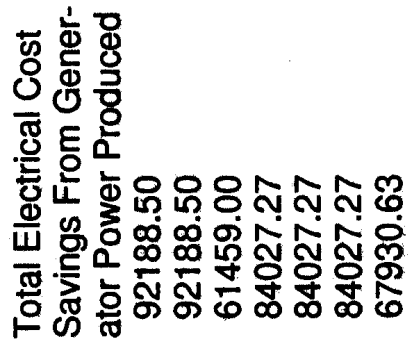

ㄴํㄴํำ

गं\%の

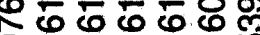

ळ 


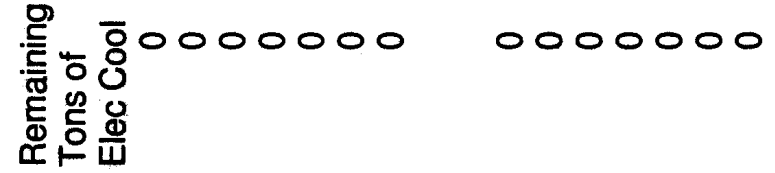

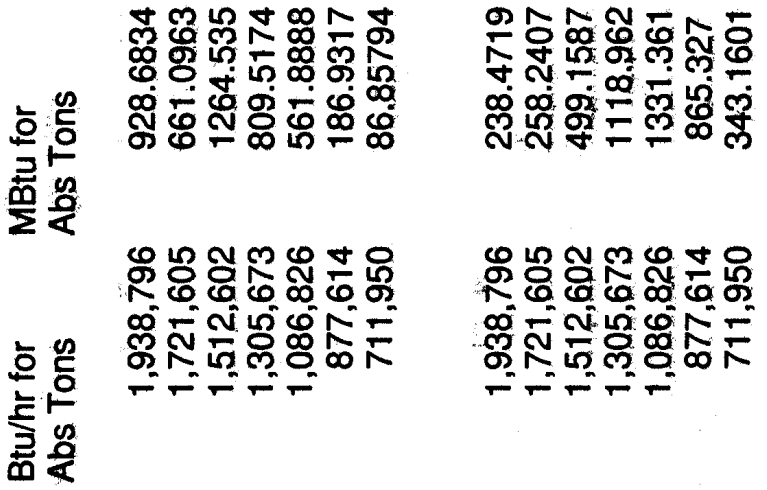

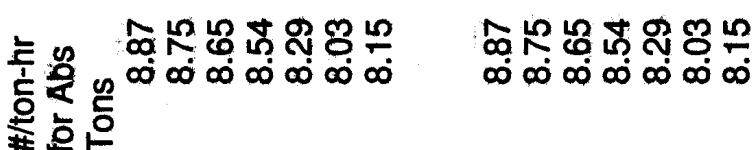

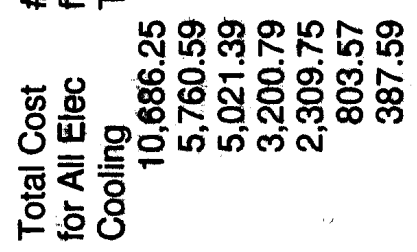

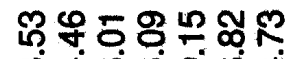

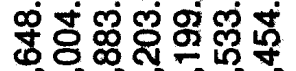

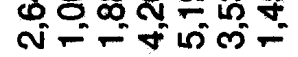
정 흥응
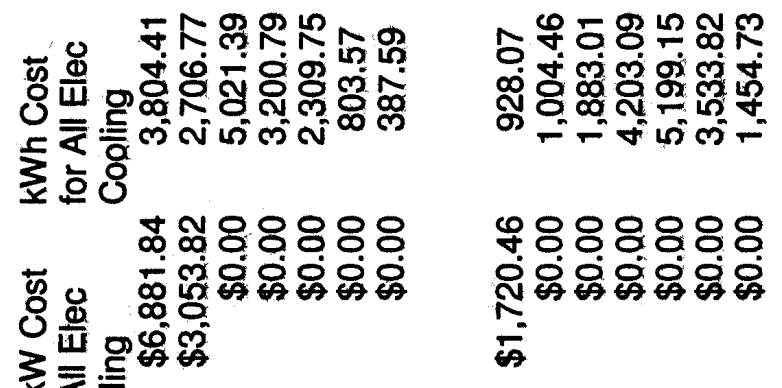

흥ㅇ
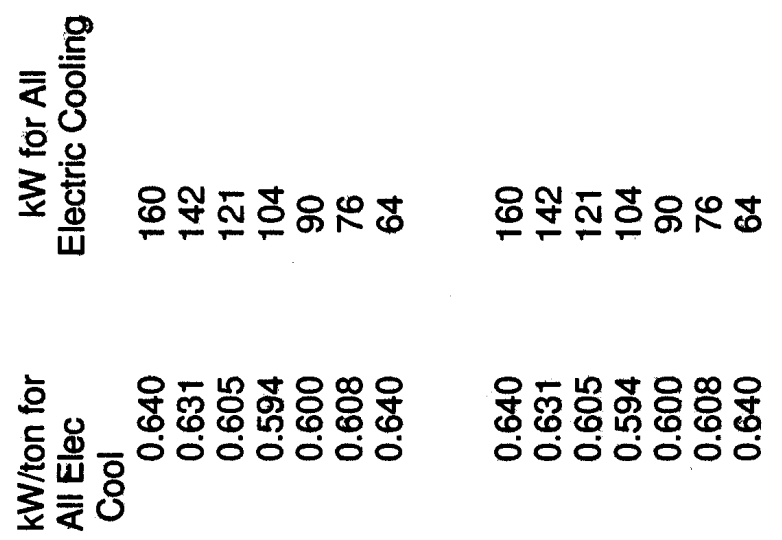

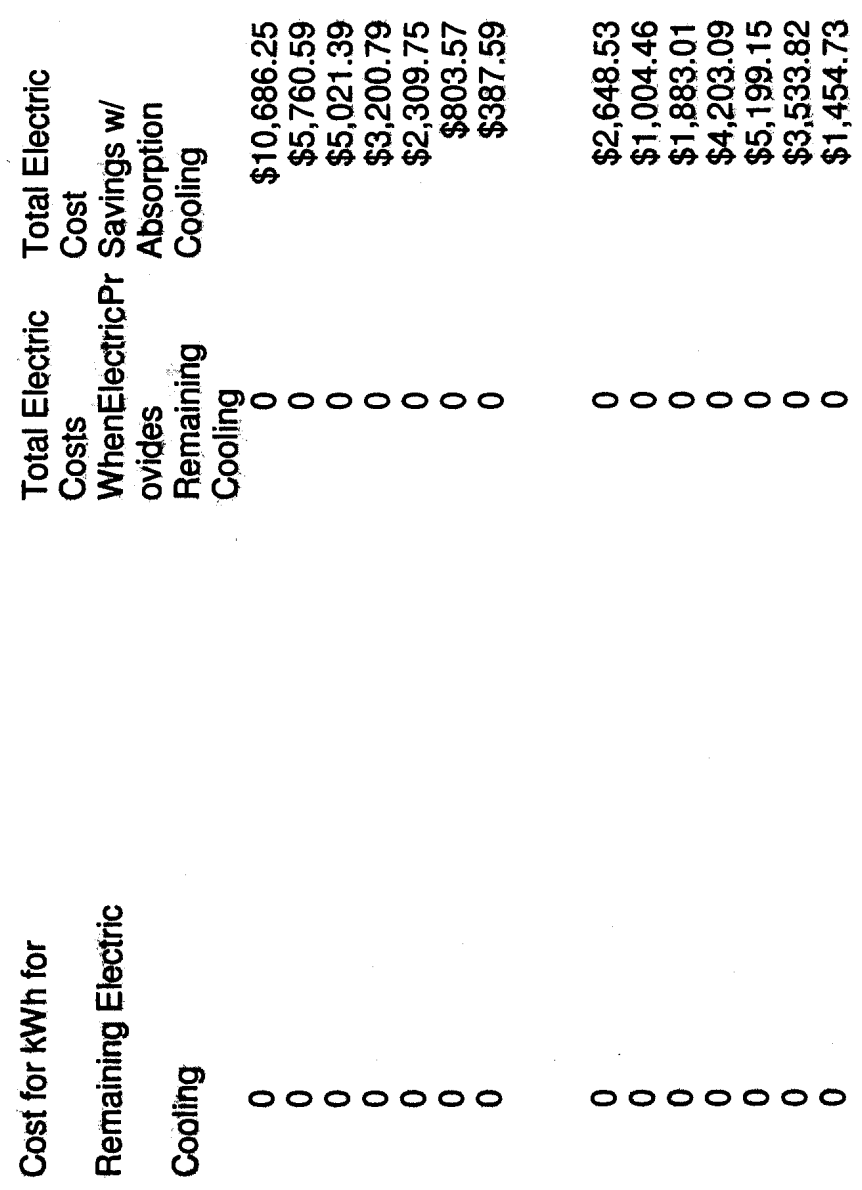

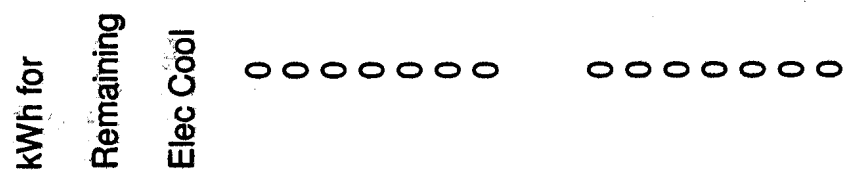

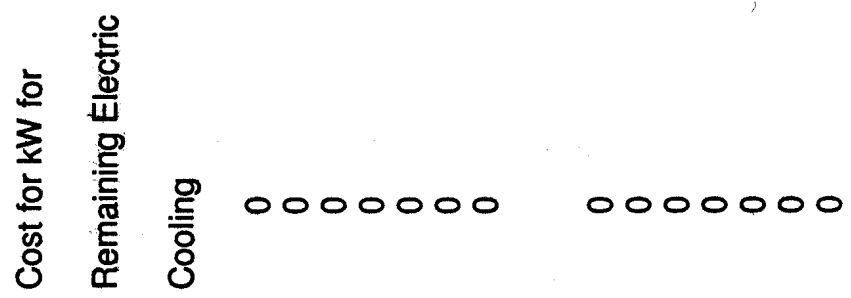

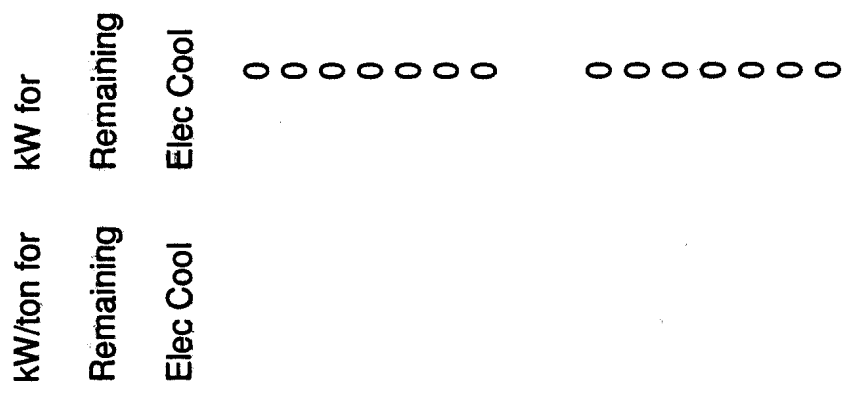




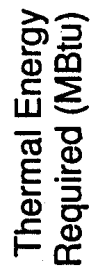

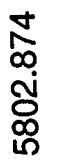

$\mid \begin{aligned} & 0 \\ & 0 \\ & 0 \\ & \\ & 0 \\ & 0\end{aligned}$

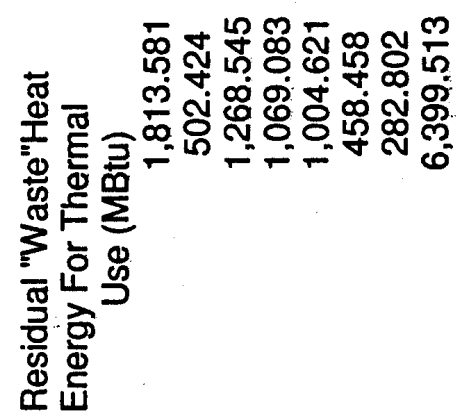

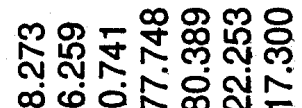

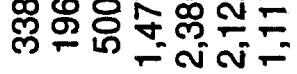

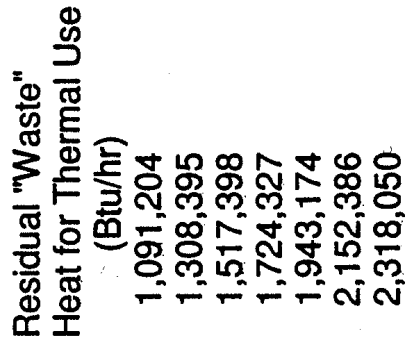

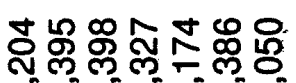

-

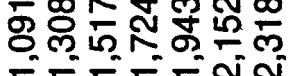

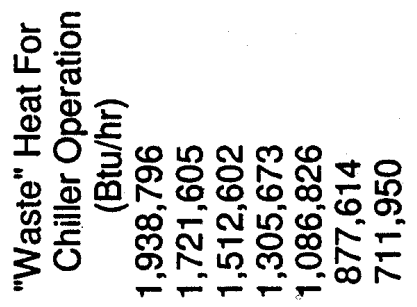

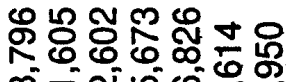

$\infty$ -

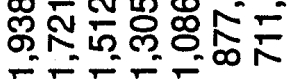

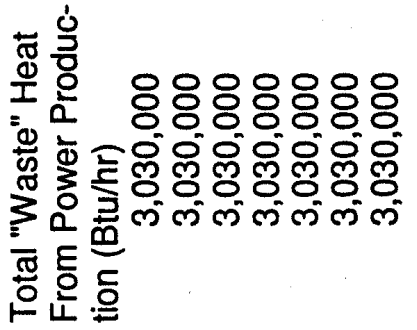

영ㅇㅇㅇㅇㅇㅇㅇㅇ

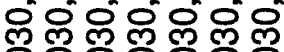

0

ले लं लं लं

흉한은 

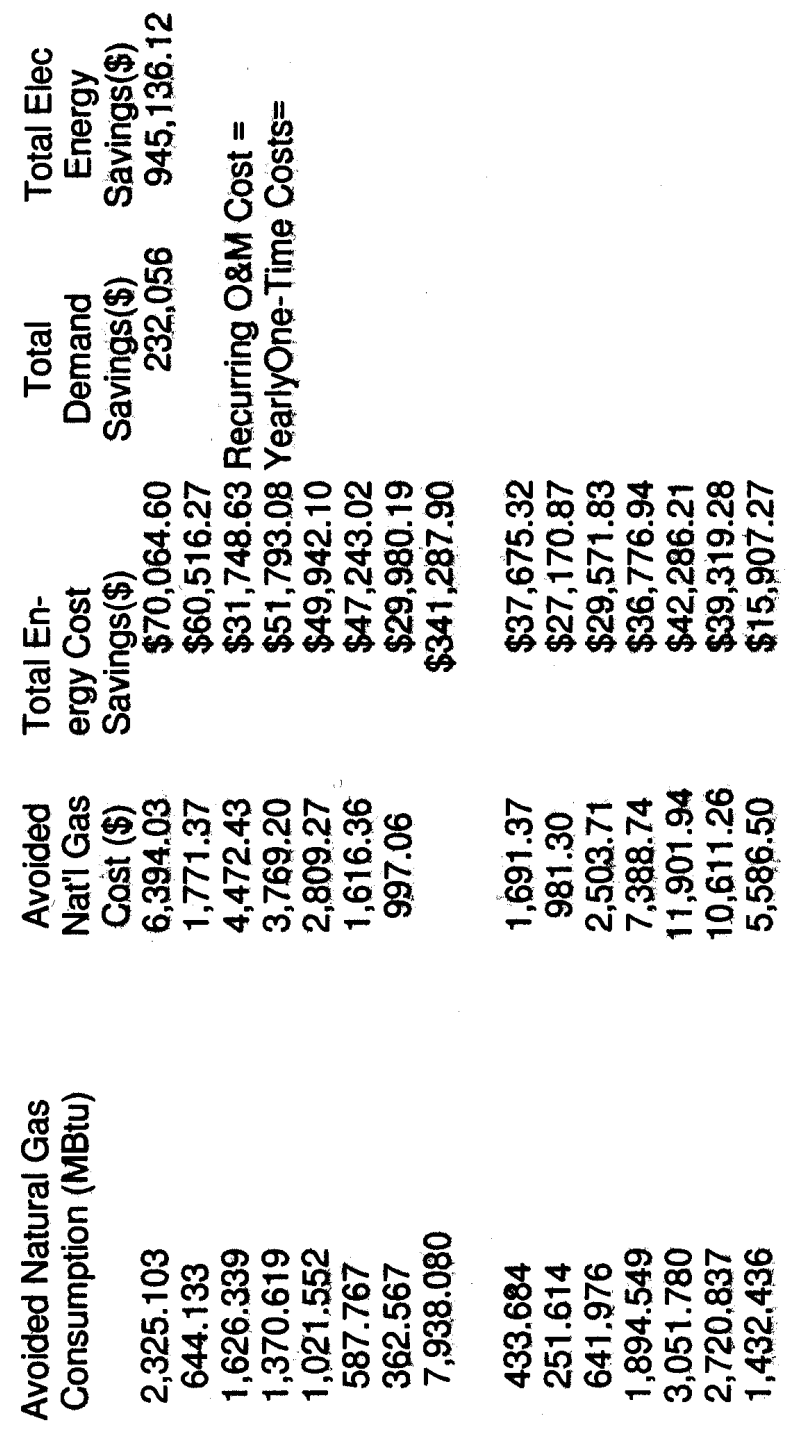

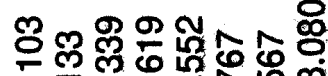

T.

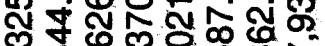

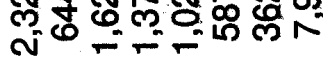

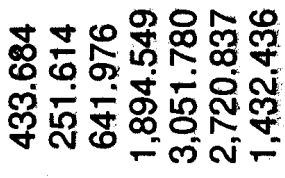

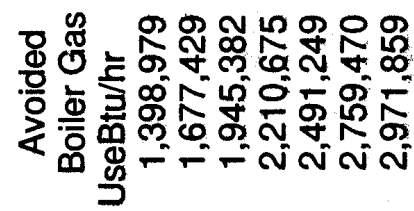

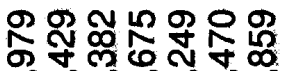

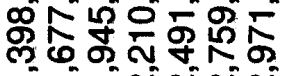

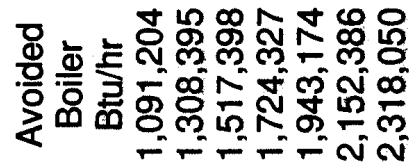

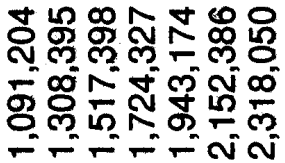




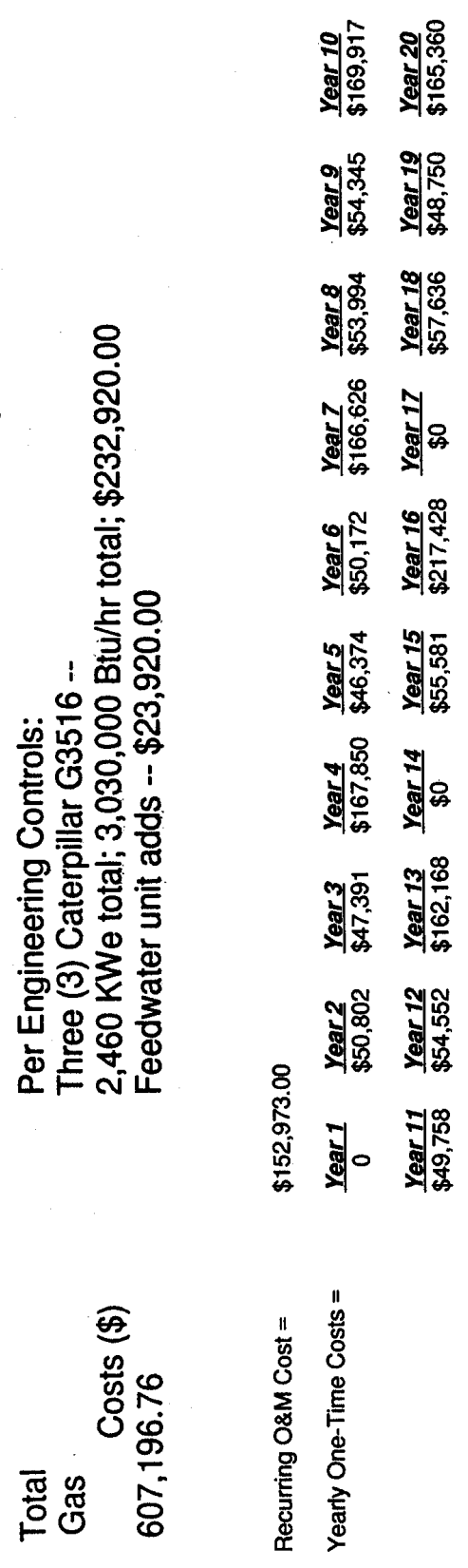




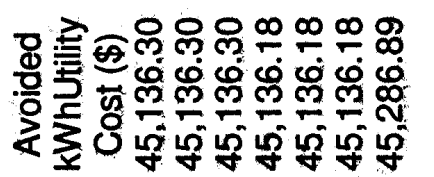

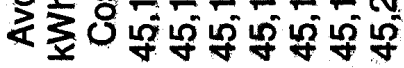
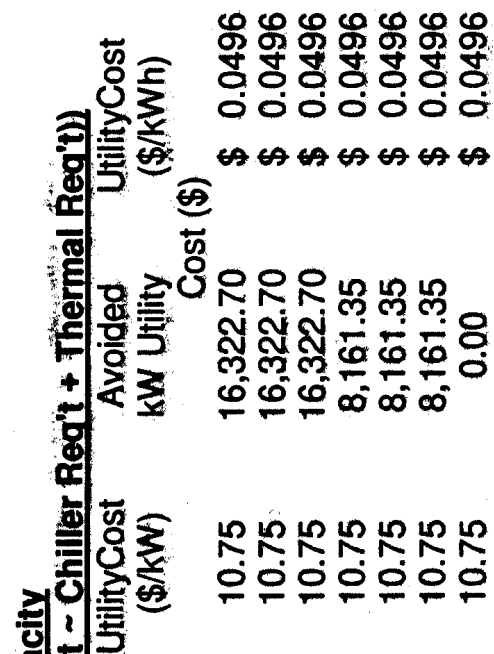

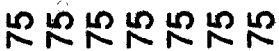
으응ㅇㅇㅇㅇㅇㅇㅇㅇ

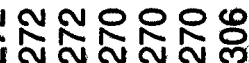
ㄴ.

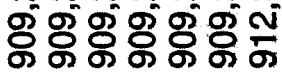
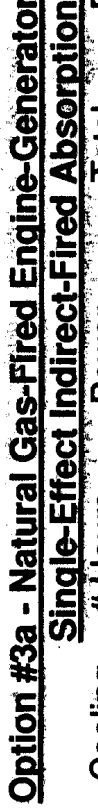
갬윰윰윰윰유
두웡영ㅇㅇ

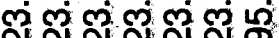
กิ่

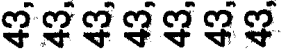

N $\mathbb{N} \mathfrak{N} \mathbb{N}$

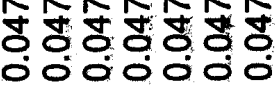

$\sec \Leftrightarrow \cos$

웅 ô ổ

สิ่ มิ่ ช่ำ

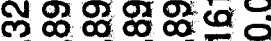

응ㅇㅇ으으

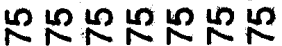
응으응ㅇㅇㅇㅇㅇㅇㅇ

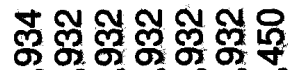

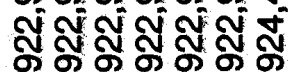

융융융융용

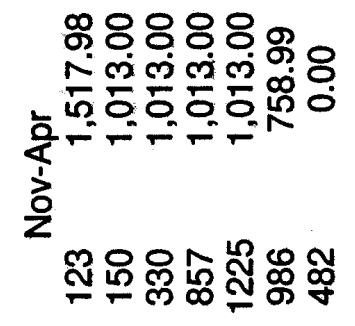

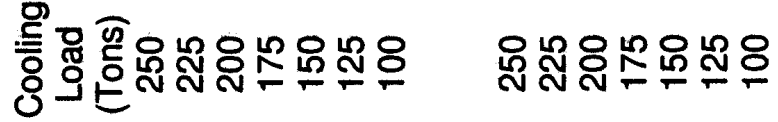


엉

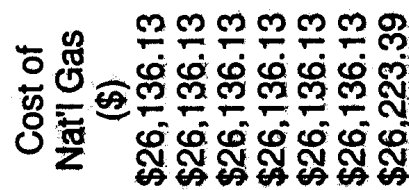

ச요용

สิ่ พิ่ พิ่

๑o co co

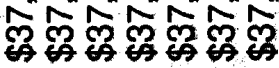

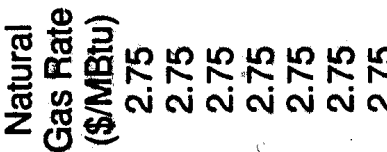

৪্용ㅇㅇㅇㅛ लं लं लं लं

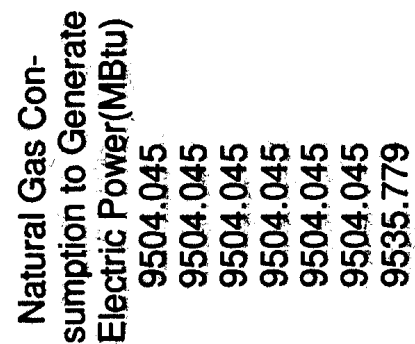

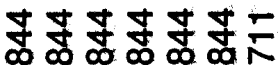
ஸ்

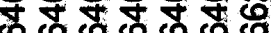

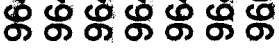

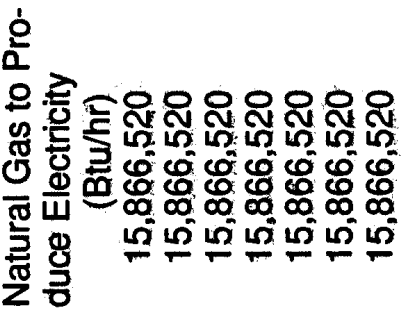

유윳유원저 กิ : $808 \%$ $\infty \infty \infty \infty \infty$

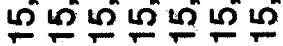

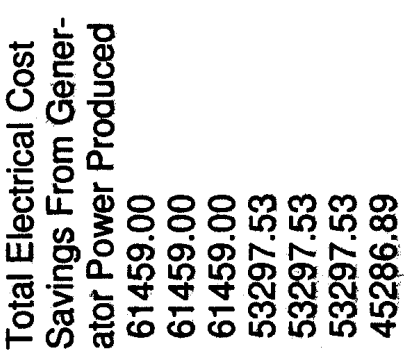

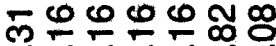
ம் ம் ம்

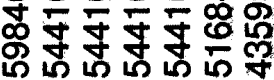




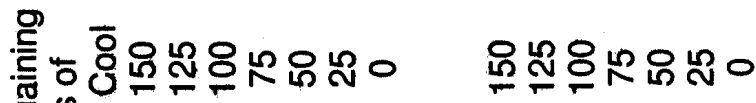
递要㐘

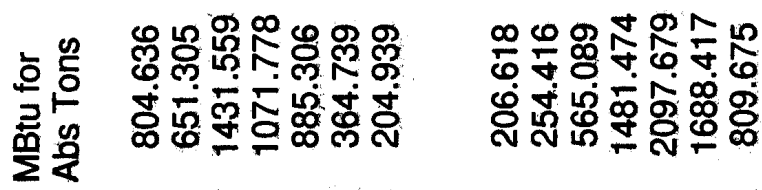

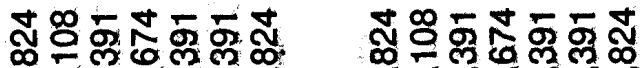

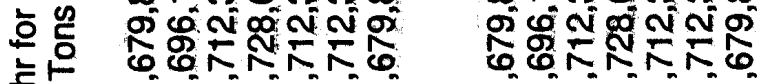

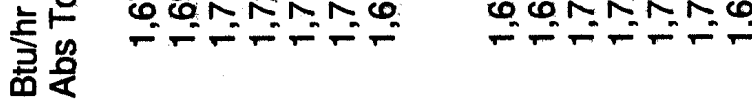

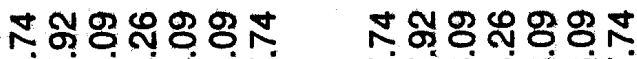

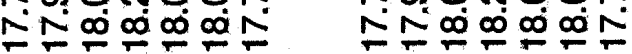

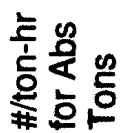

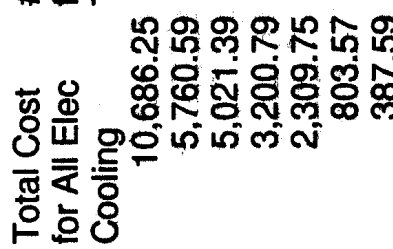

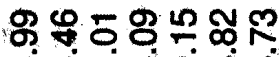

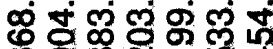

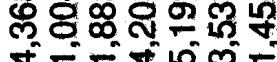
ठㅍำ 항ㅇㅇ

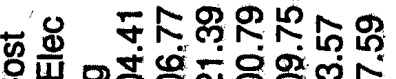

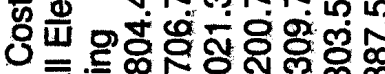
₹产 产高

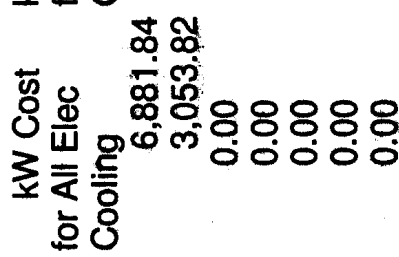

승 웅욘뜌

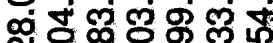
ชั่으문

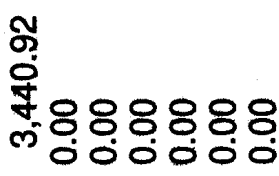

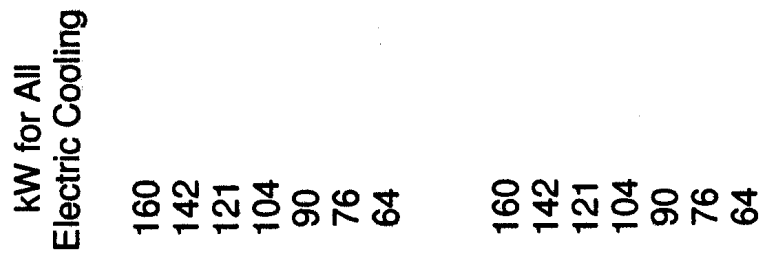

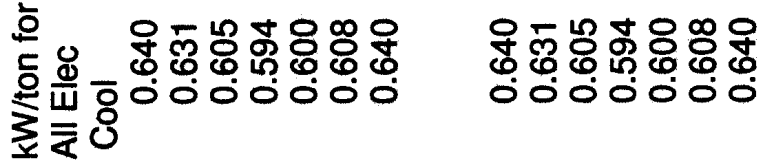



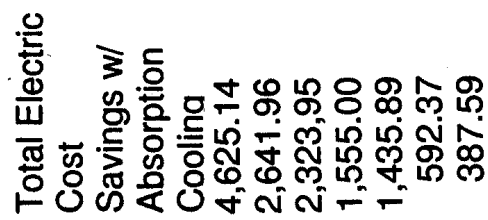

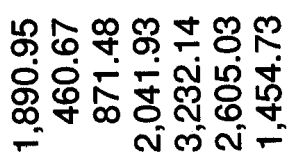
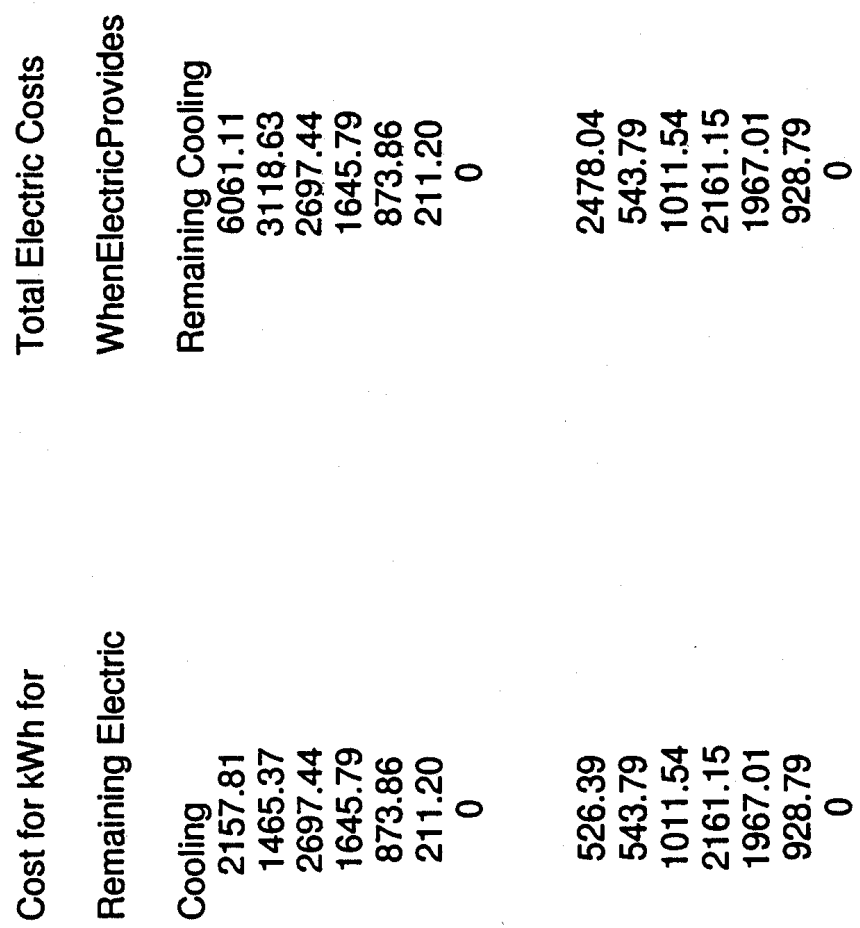

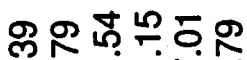

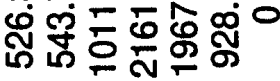

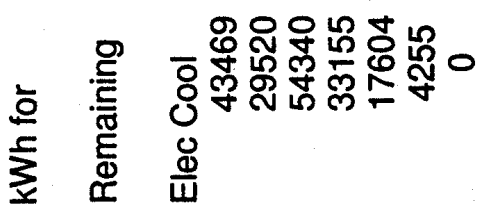

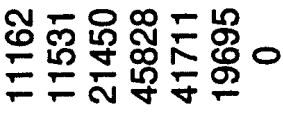

$\frac{\grave{2}}{\frac{2}{2}}$

인요

은 लำ

잉융

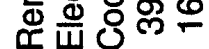

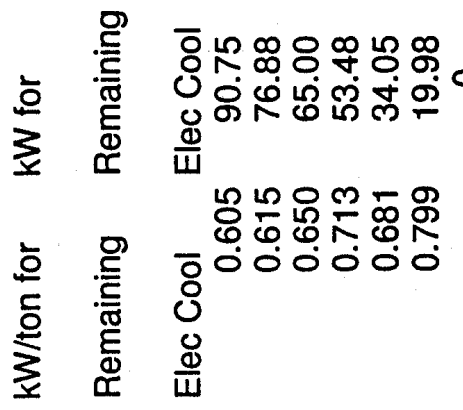

号 000000

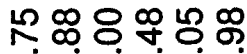

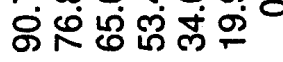

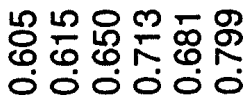



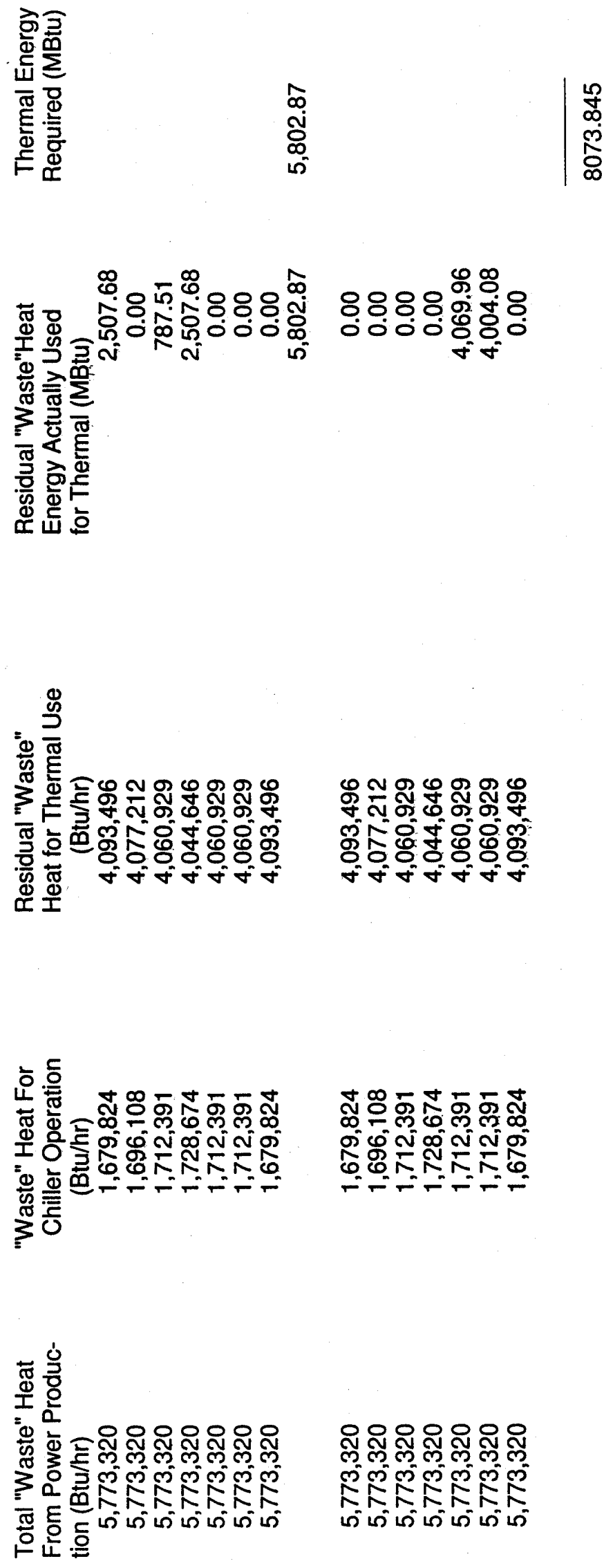


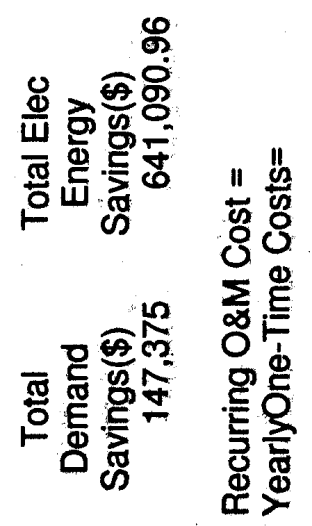

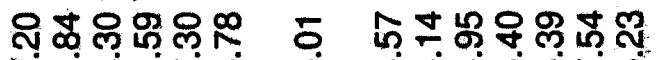

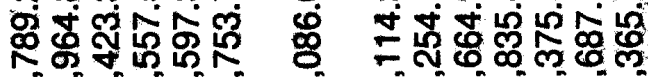

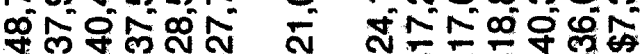

㐫

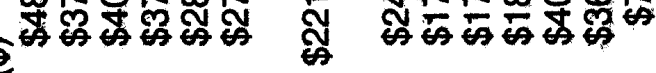

응 홍

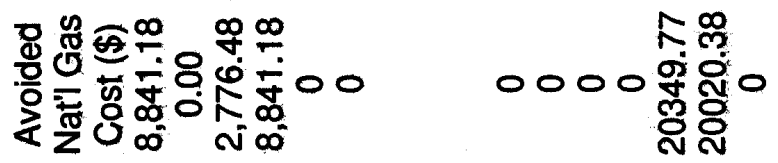

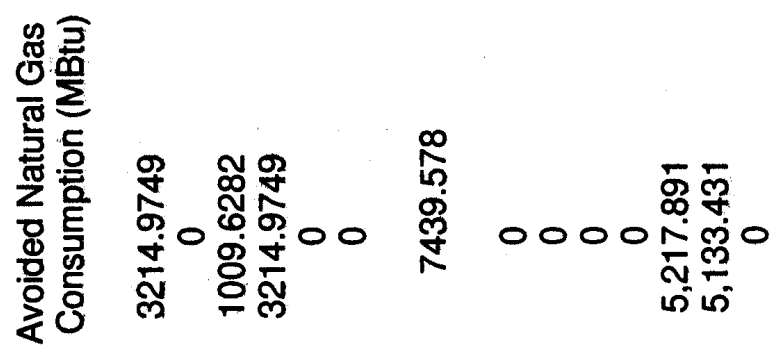

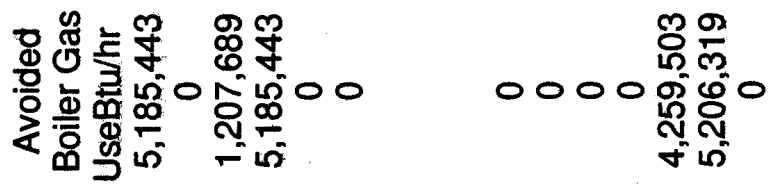

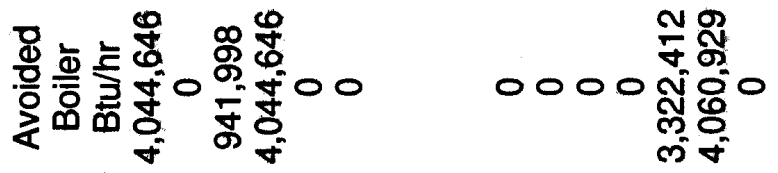




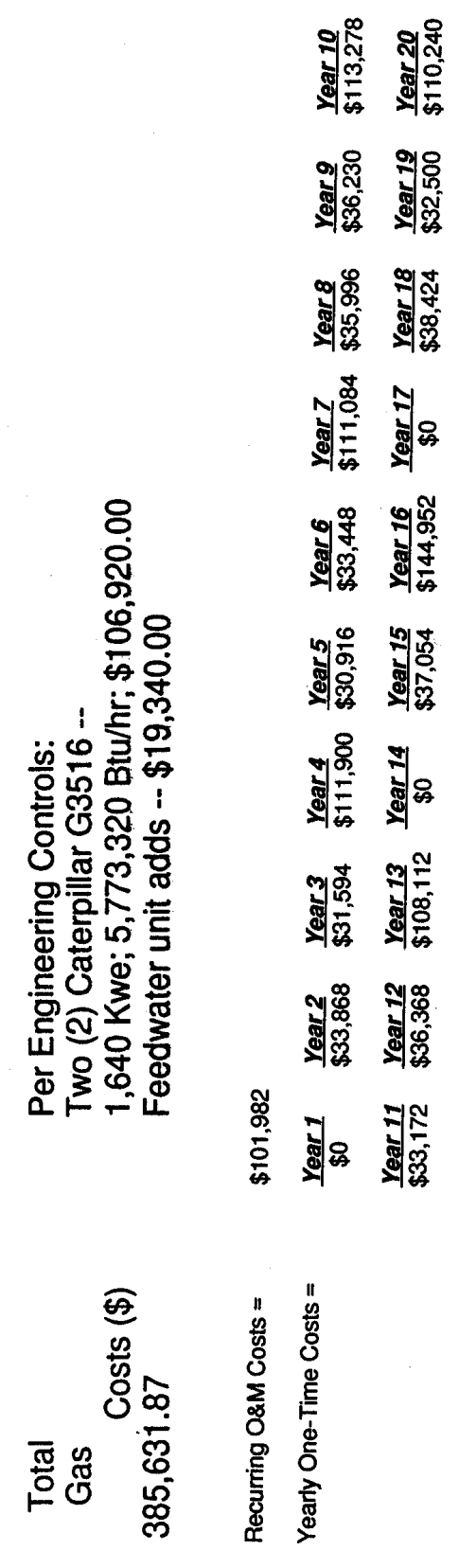




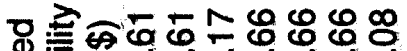

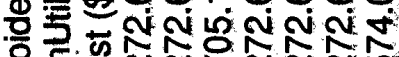

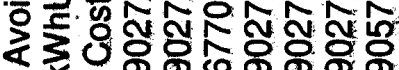

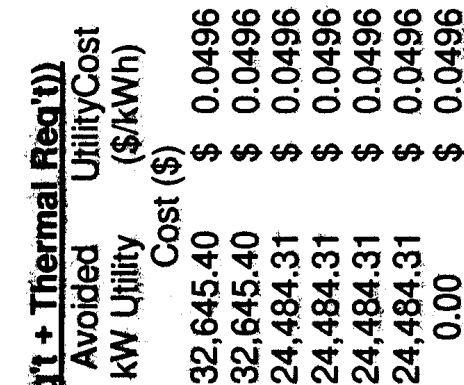

ปั o.

항 용

of

희의

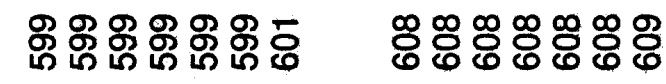

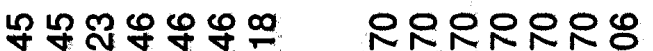

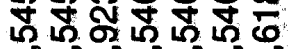
$\infty \infty^{\circ} \infty \infty \infty$

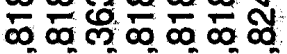

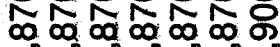

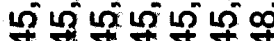

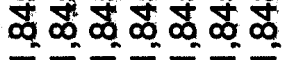

ษับุ̣

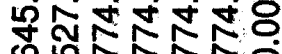

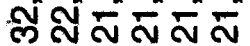

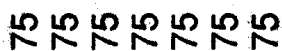

으으유으으응으

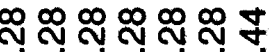

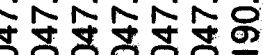

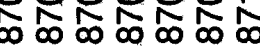

NNNNNNN

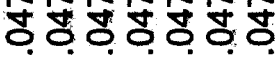
०000000 -

$T-F-T$

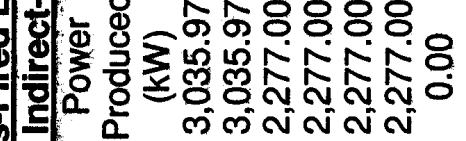

$$
\begin{aligned}
& \text { 5. } 88888
\end{aligned}
$$

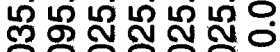

$$
\begin{aligned}
& \text { ㅇㅇㅇㅇㅇㅇㅇㅇㅇ }
\end{aligned}
$$

들

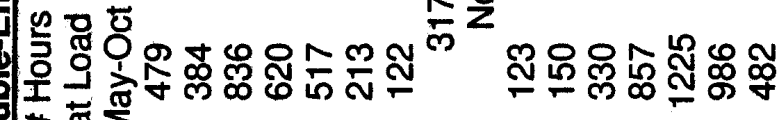

으무

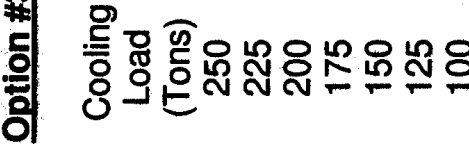

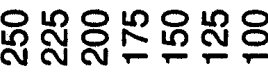




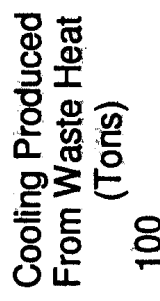

으유유으유유으 으으유으유유우

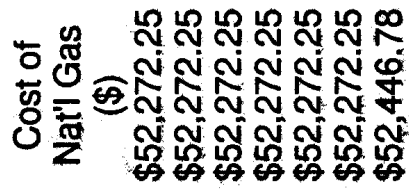

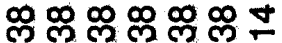

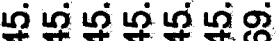

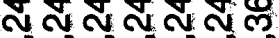
ก

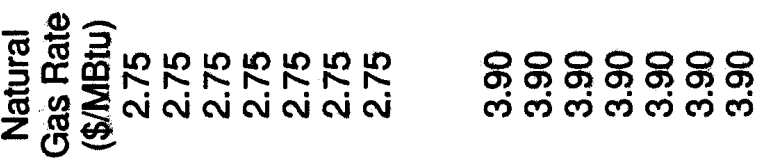

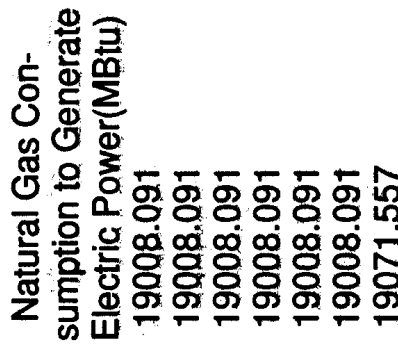

జొ 60 o. รูㅉํำ

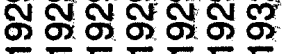

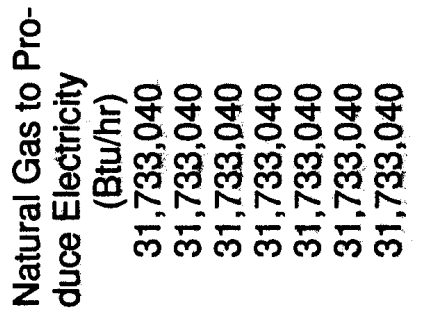

앙웡원영영영

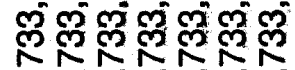
लं लं लं लं ले

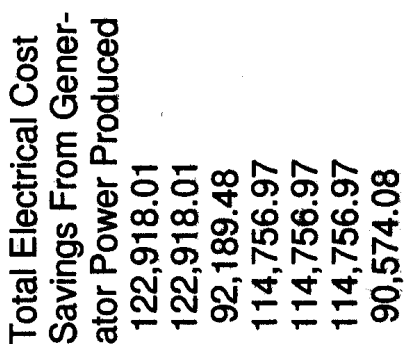

유요요묘

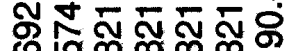
네 000 뭉ㅇㅇㅇㅇㅇㅇㅇㅇㅇㅇ 

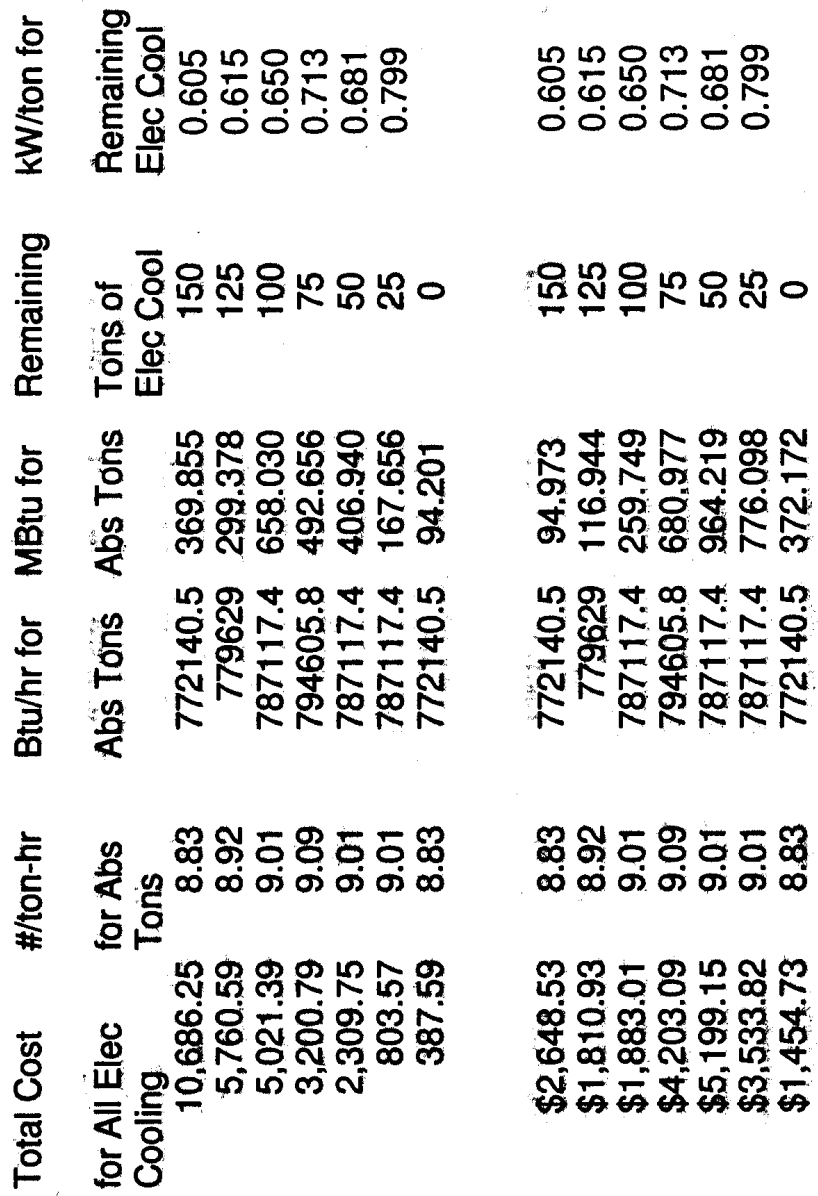

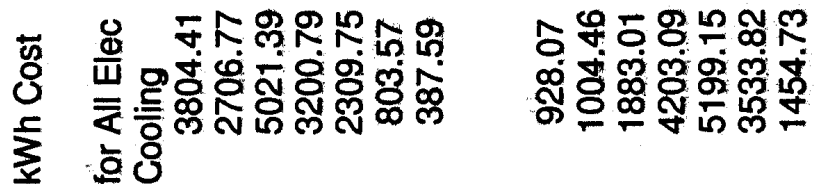

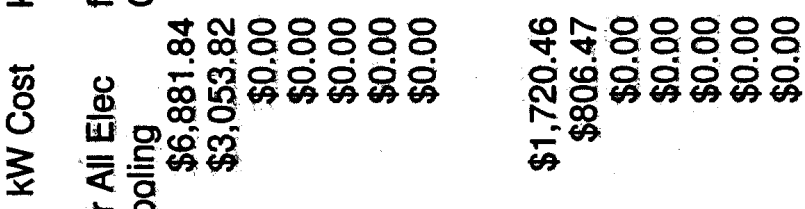
홍

$\stackrel{2}{R}$

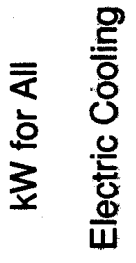

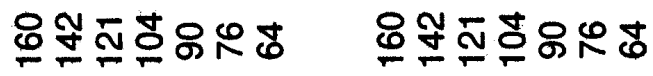

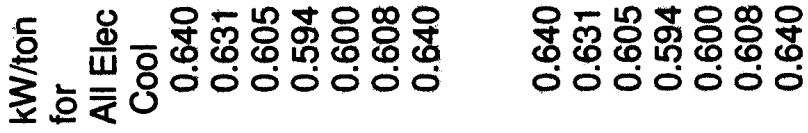



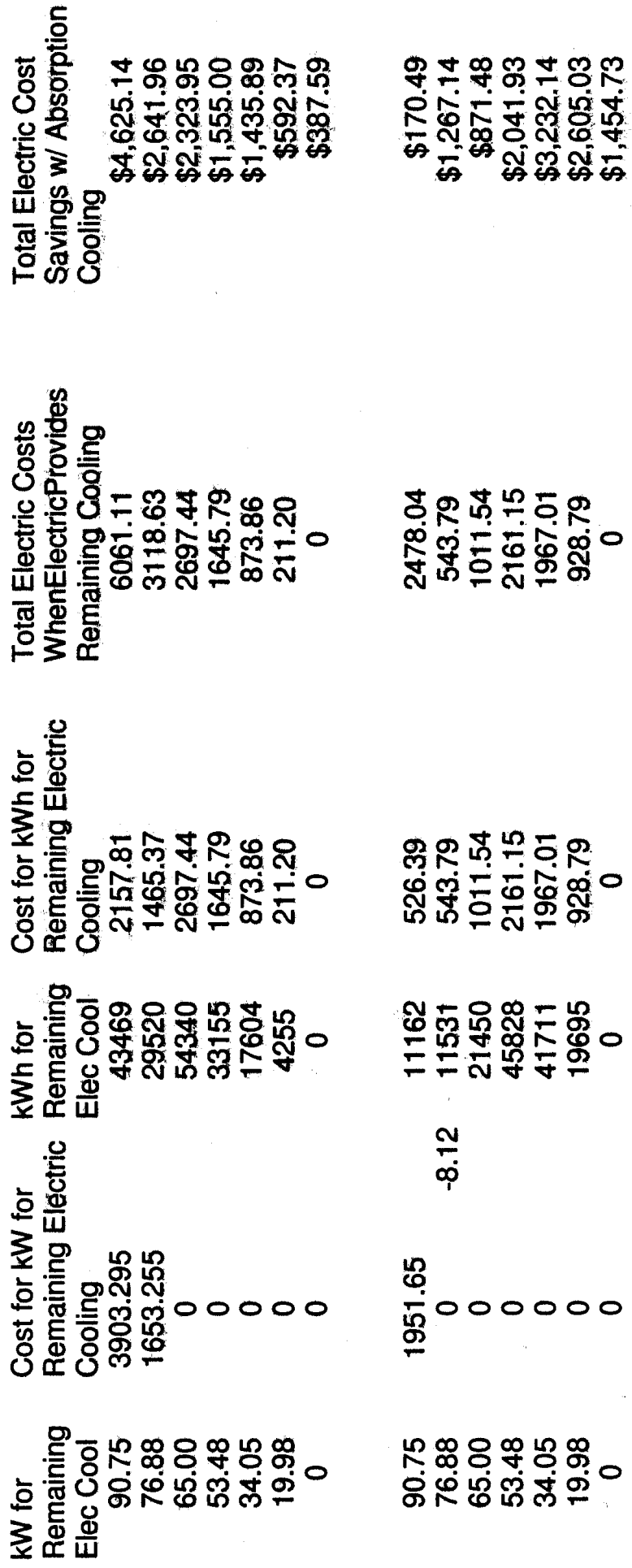


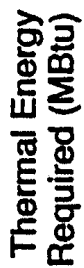

$\mathbb{N}$
$\infty$
Nิ
ه్

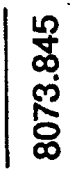

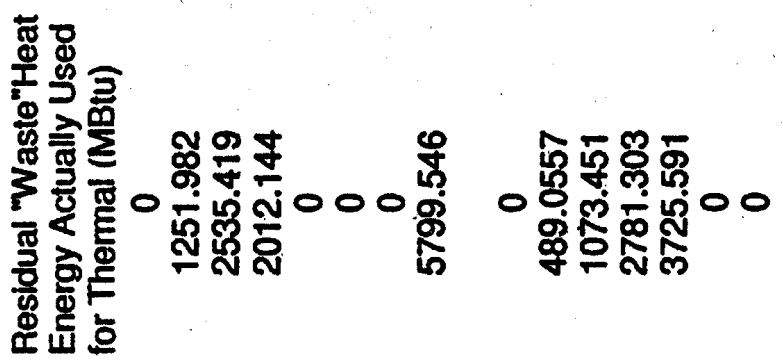
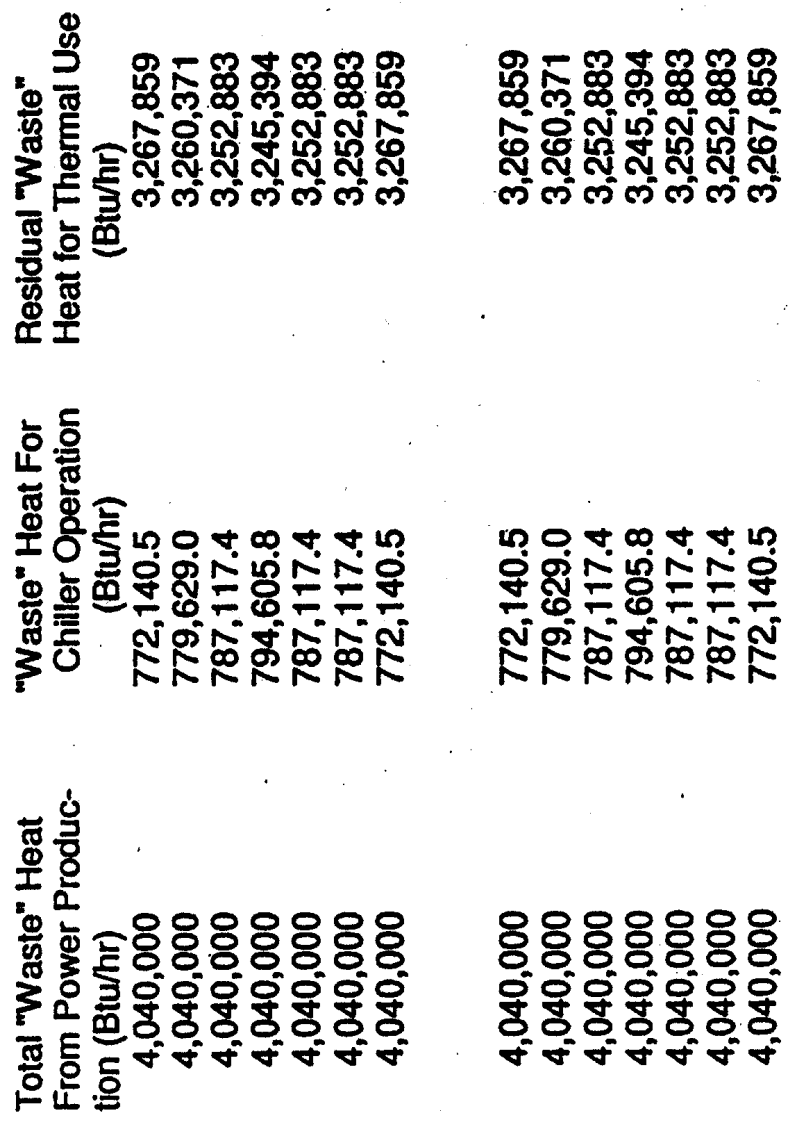

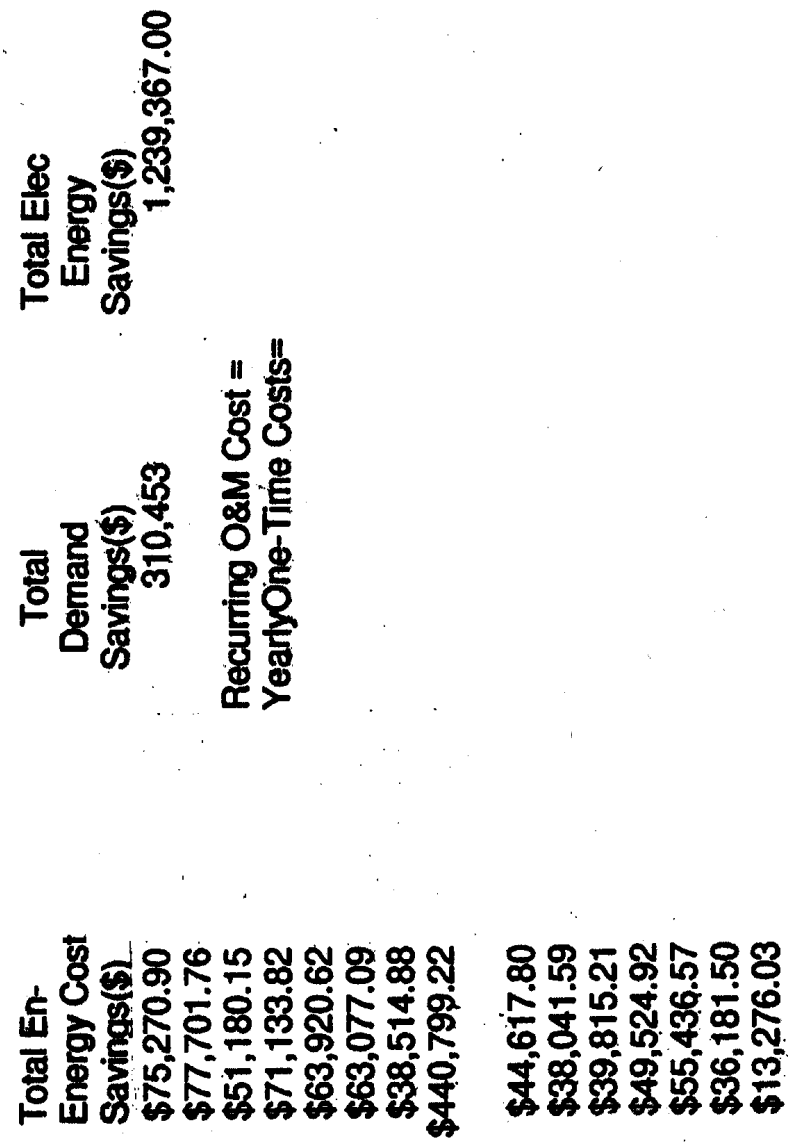

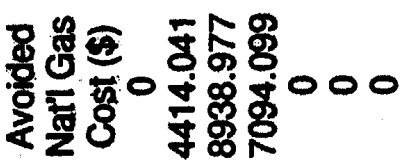
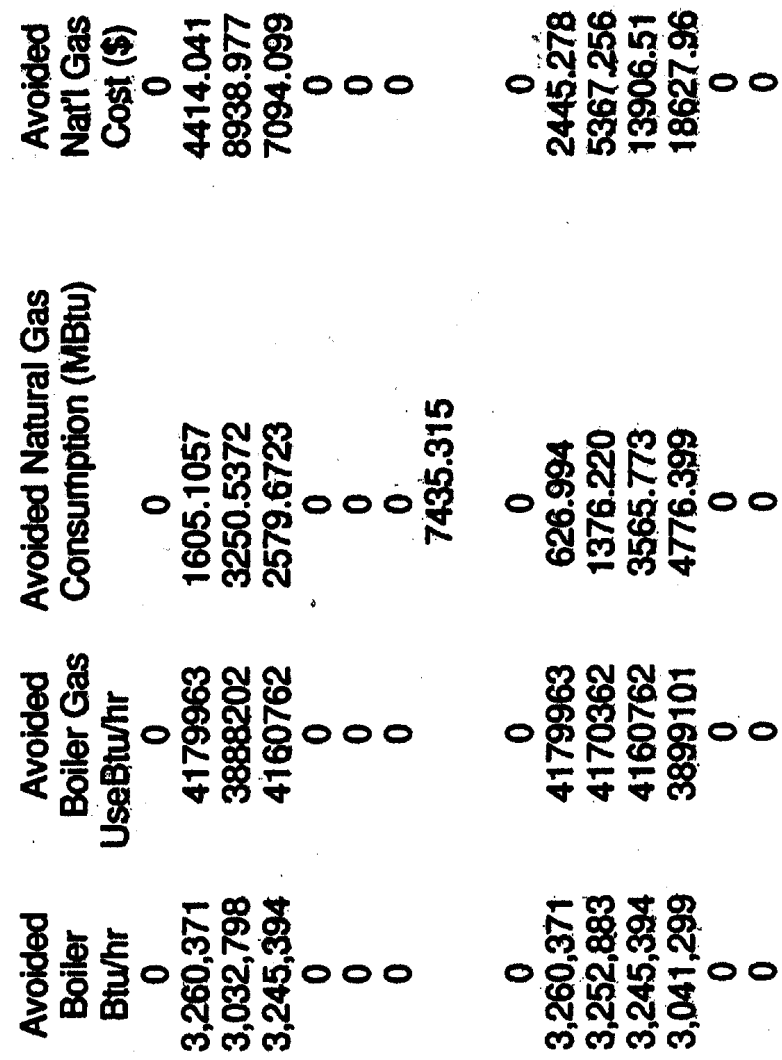


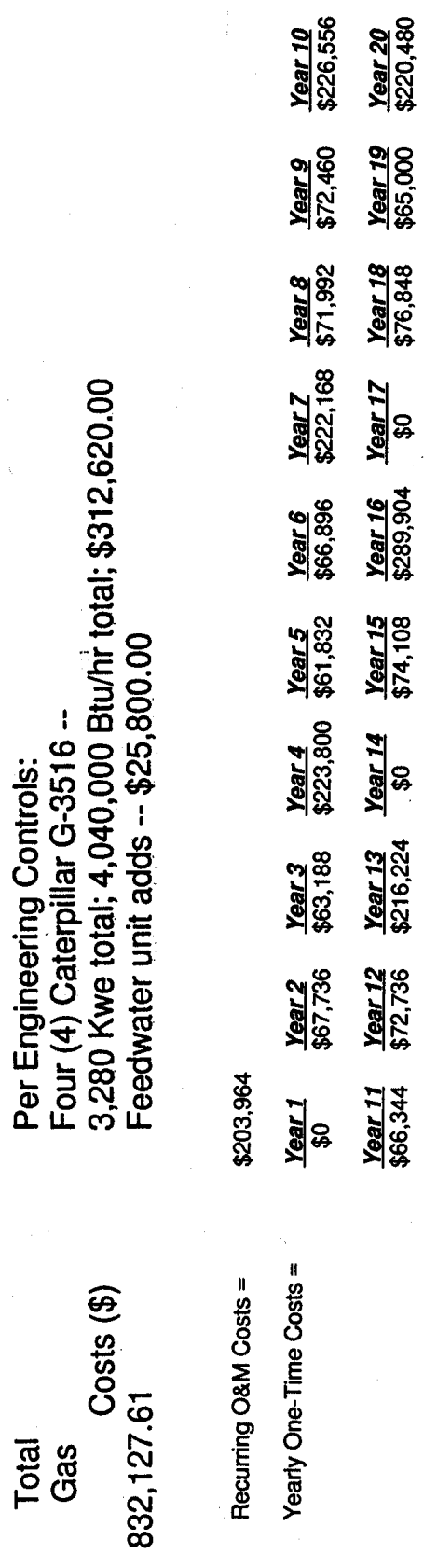




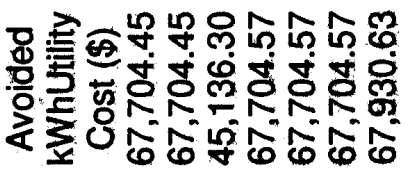

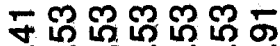
ம

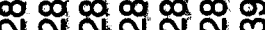

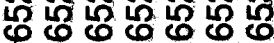

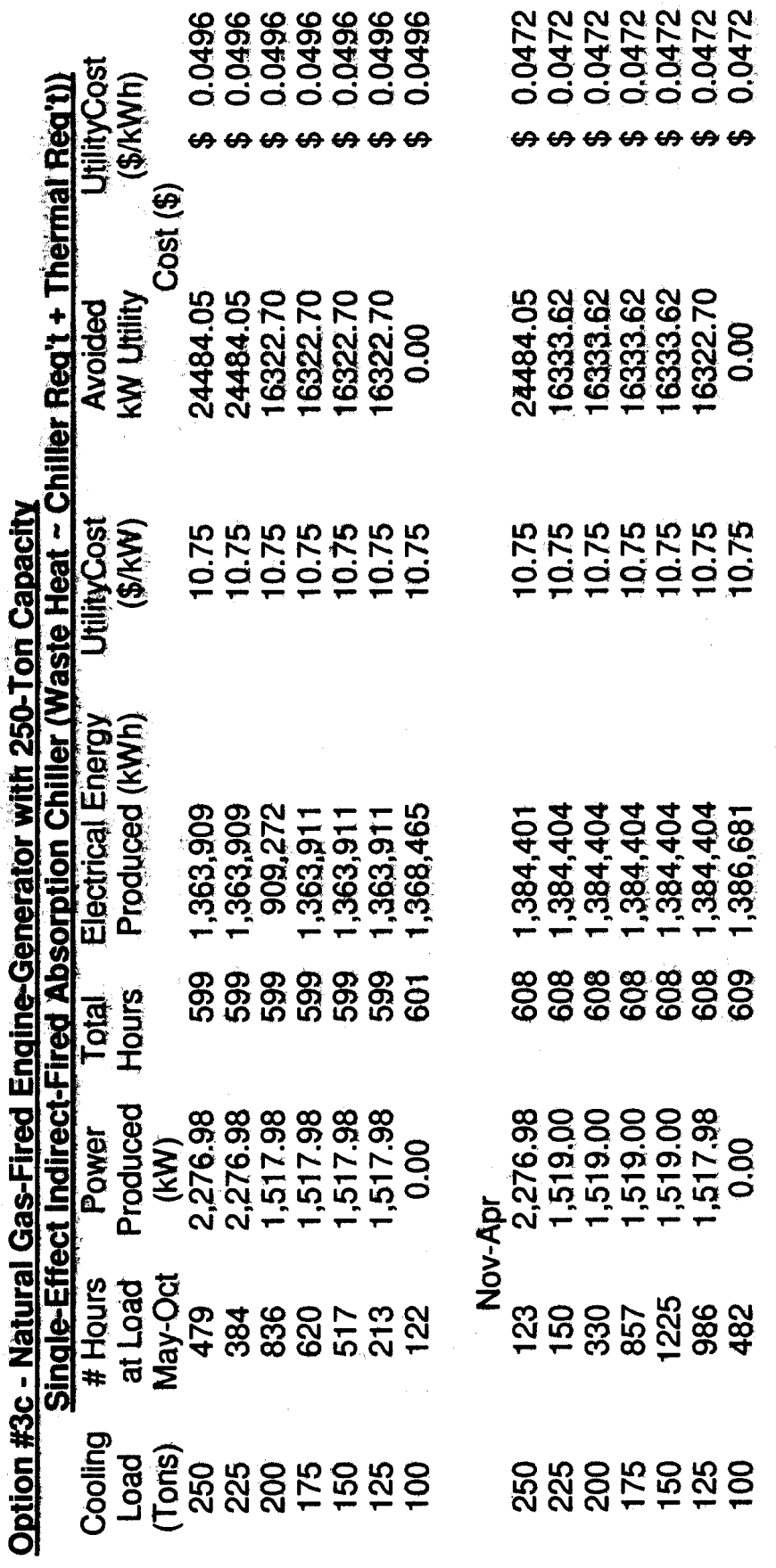


월

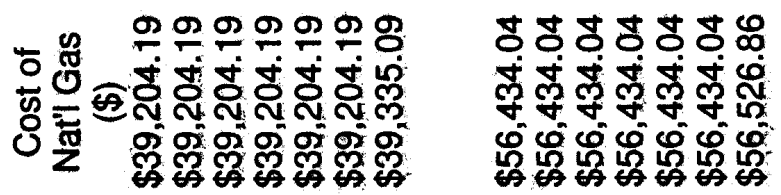

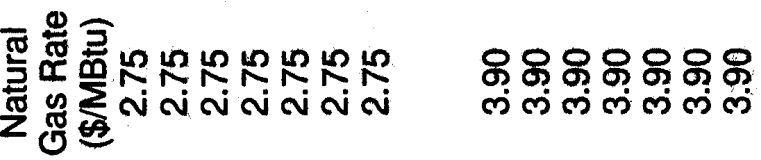

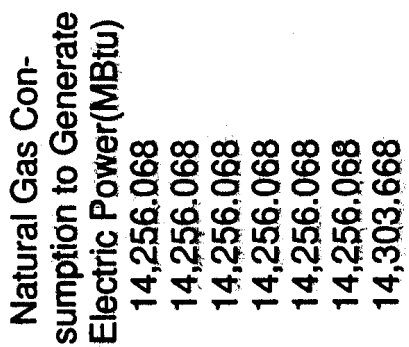

雨

र오오영

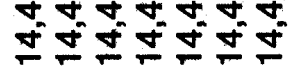

은

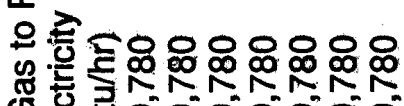

उ० पें

๘ Ш

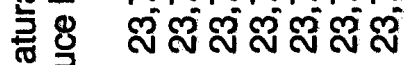

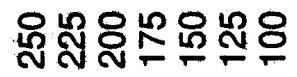

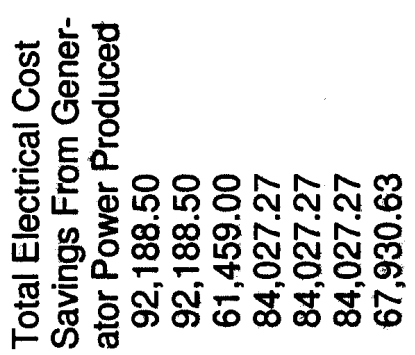

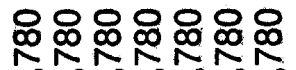

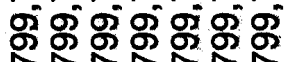
TरNTN

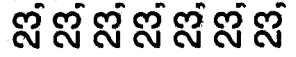

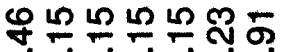

ஜ

बं 


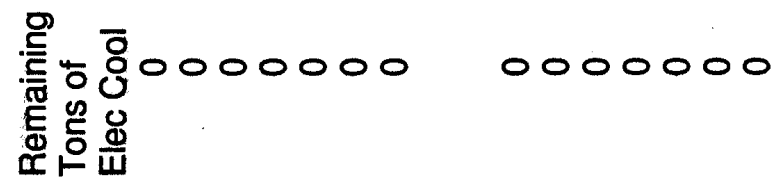

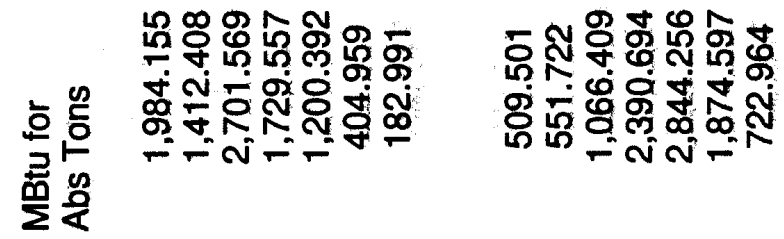

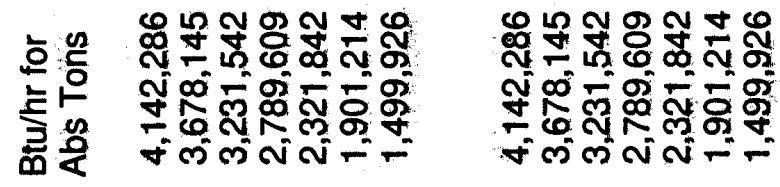

*

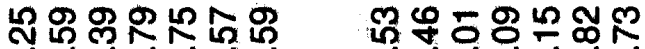

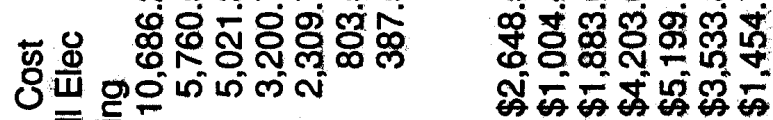

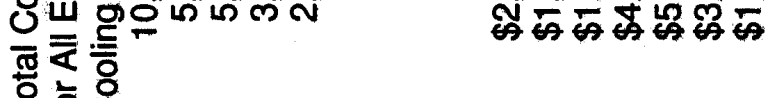

든응

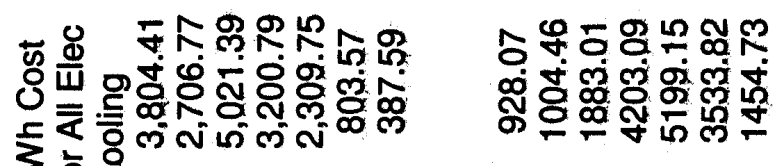
立 oㅇ

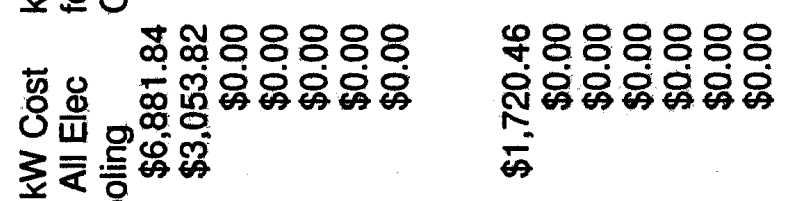

흥

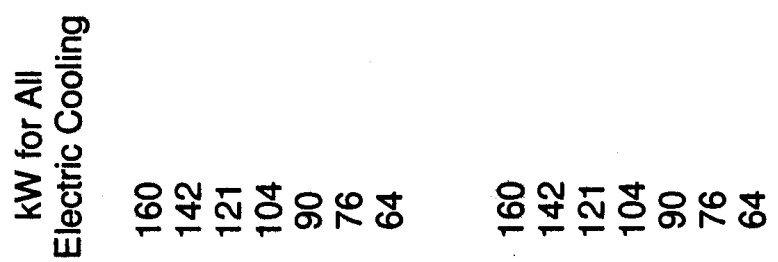

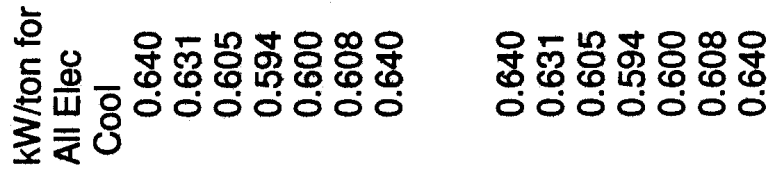



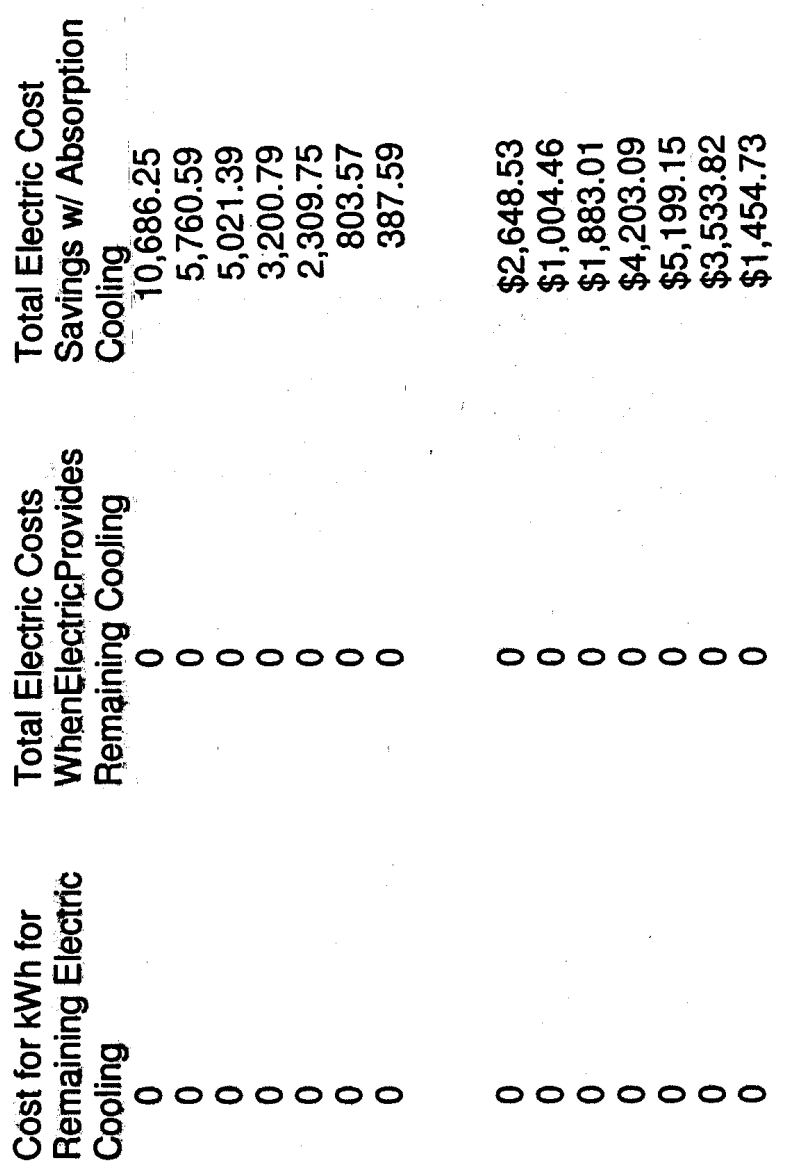

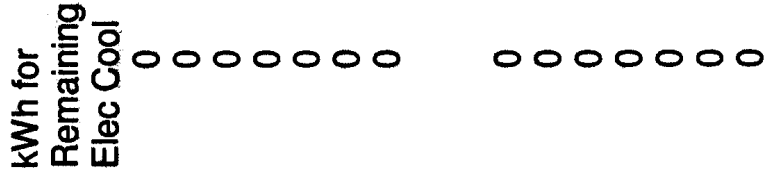

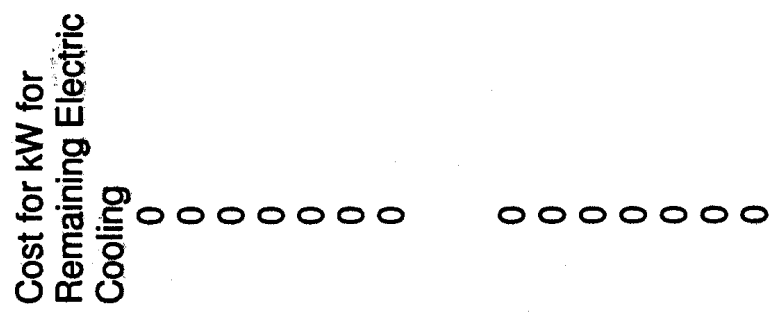

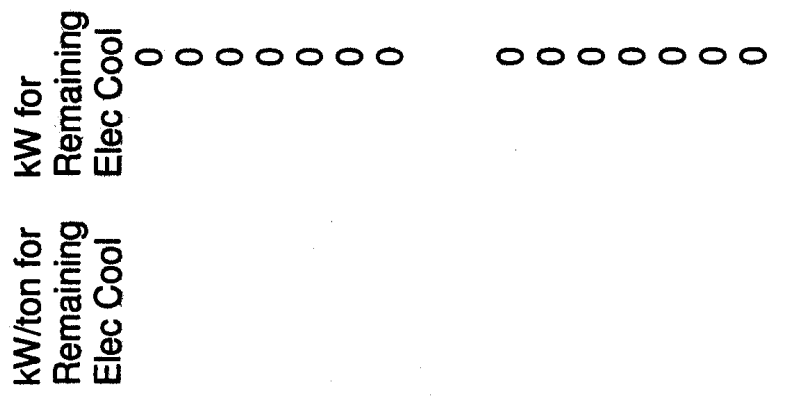



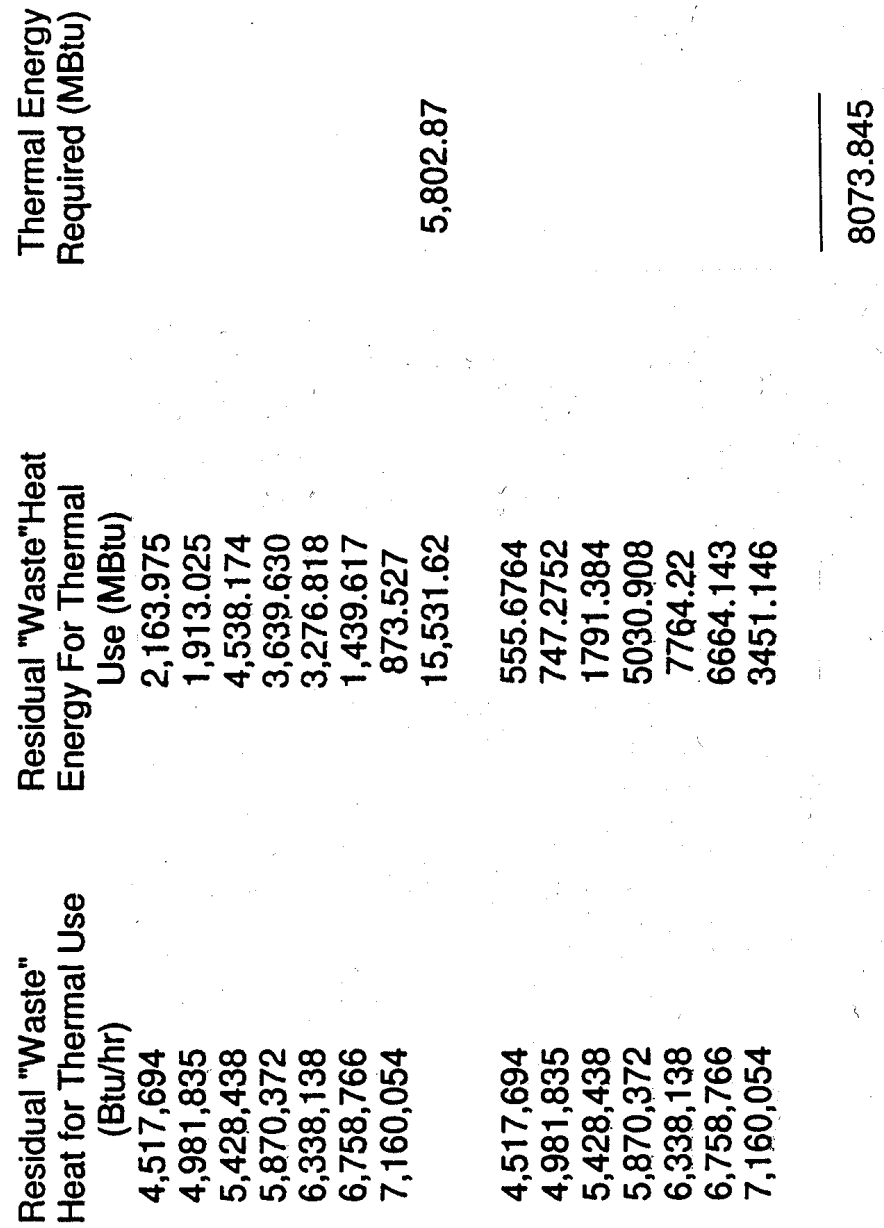

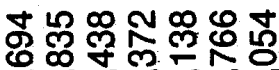
N- $0^{\circ} 0^{\circ} 0$

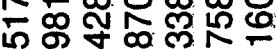

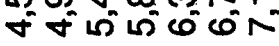
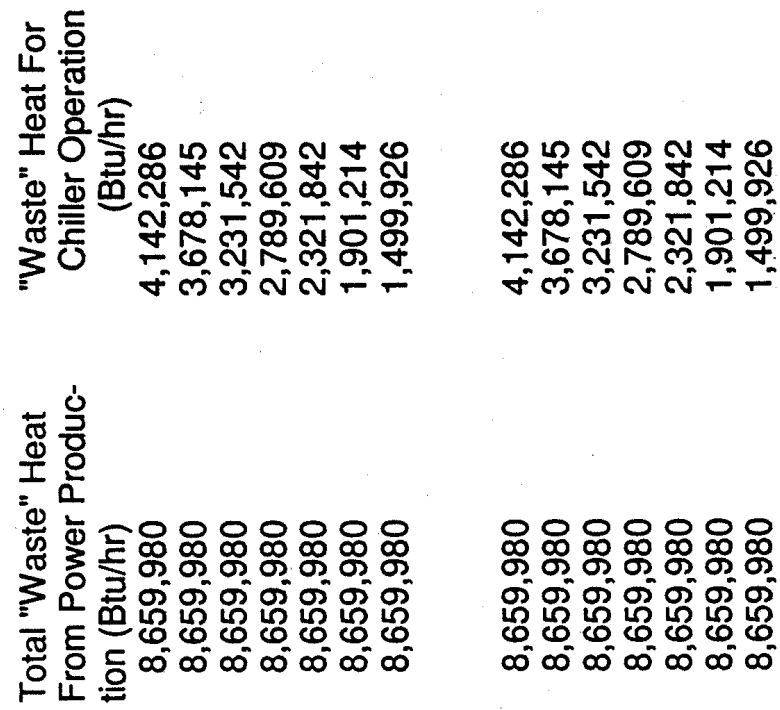

영영 영영영용

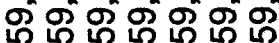
นึ $\infty \infty^{-} \infty^{-} \infty \infty^{-}$ 


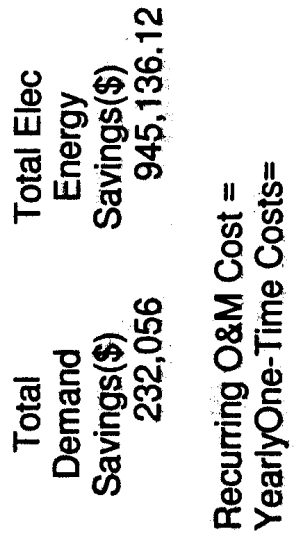

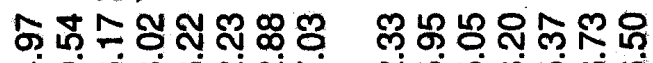

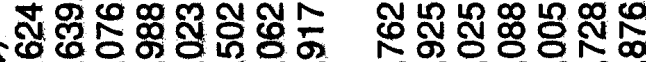

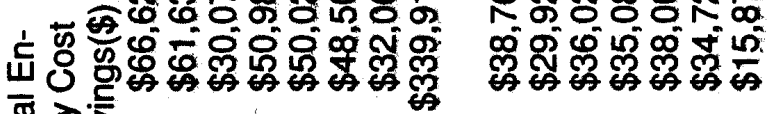

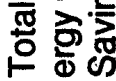

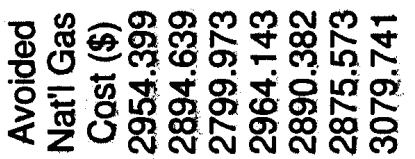

뚠ํํำ는

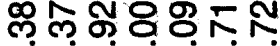

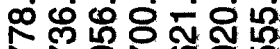

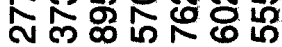

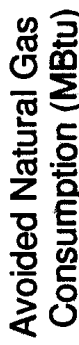

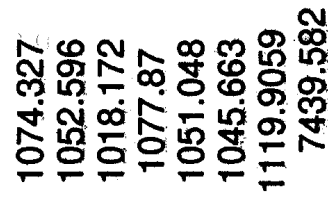

す

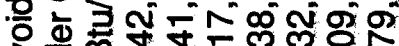

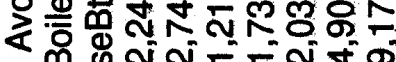
D

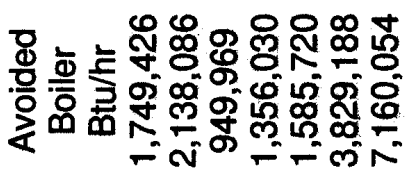

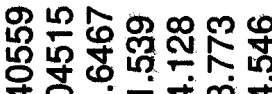
ํํㅎํ்

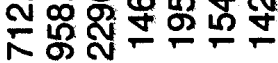

누융ํ요요 ธ్ర

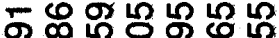

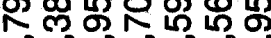
เं

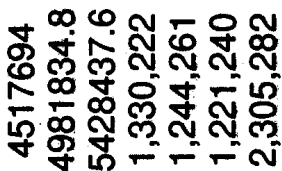




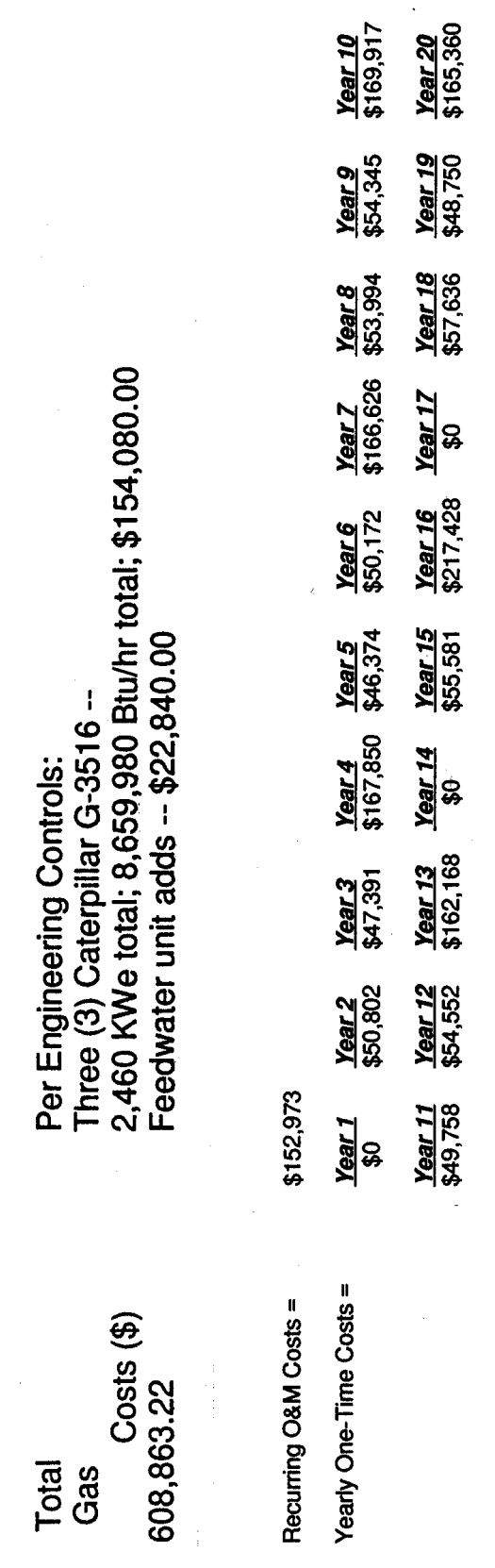




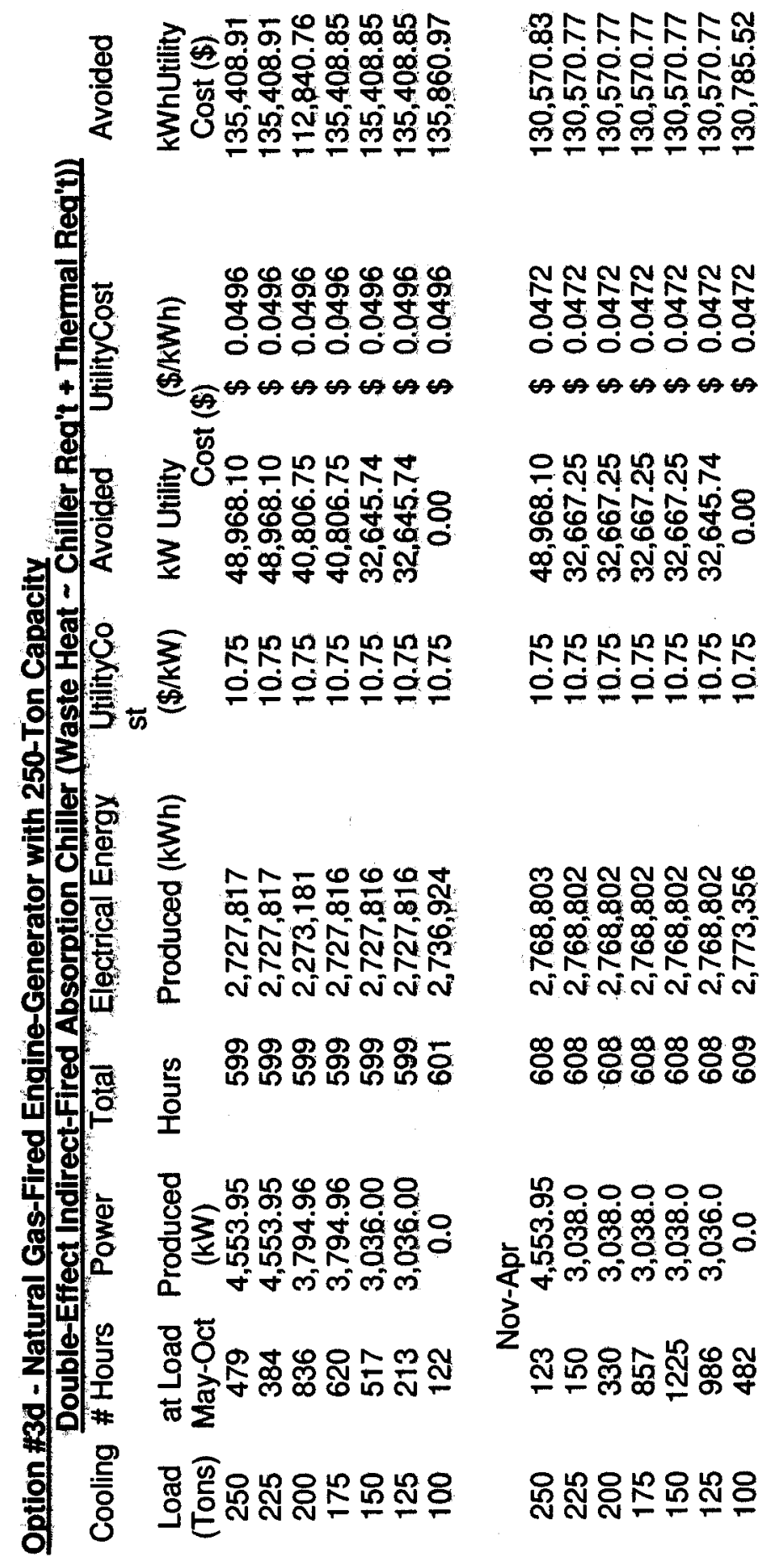



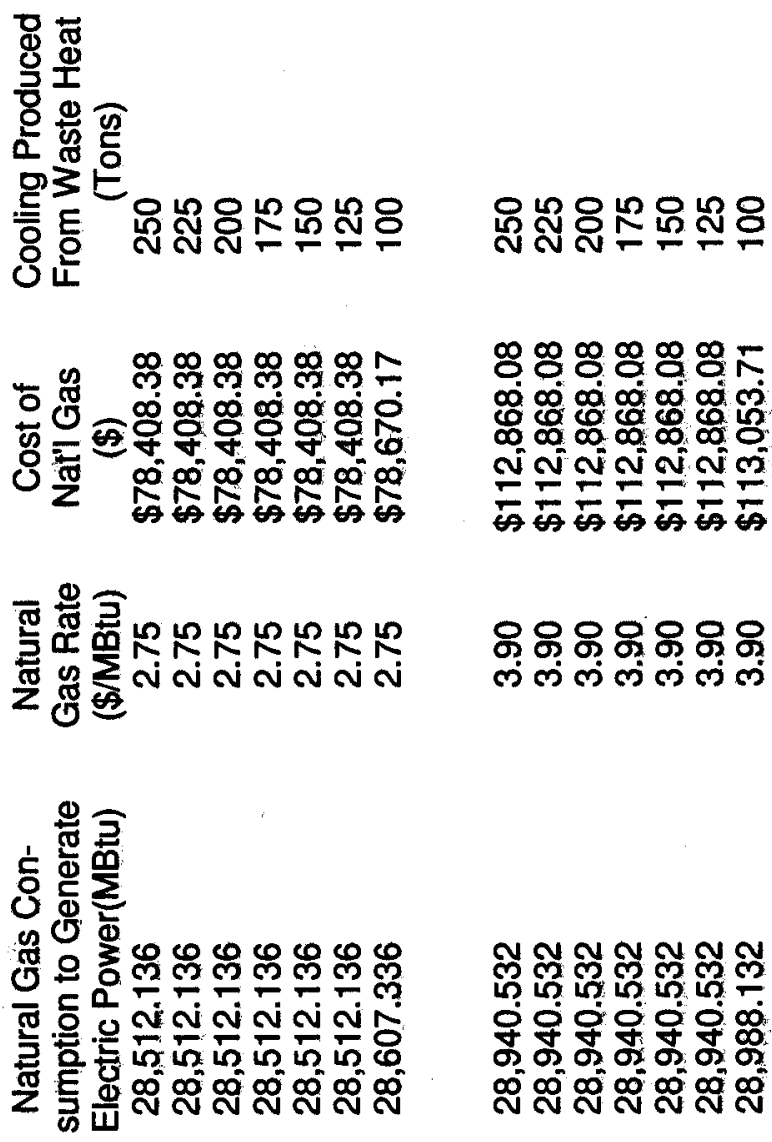

స్ స్ స్ స్ స్ స్ స్ ల్ นุ 원웡웡영 क 02 \%

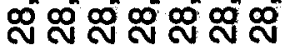

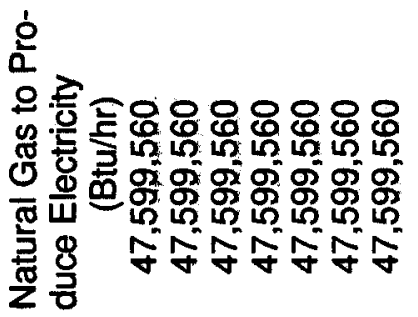

앙용요용용 นำ 요ㅇㅛㅛ용

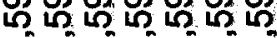
羊尔羊尔尔

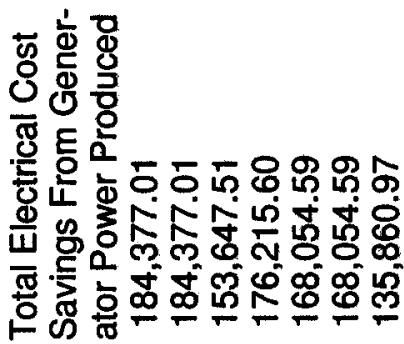

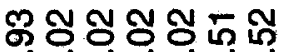
舟 గ్ల స్ స్ స్ స̃ ஜㅠ ஜீஜீஜீ 


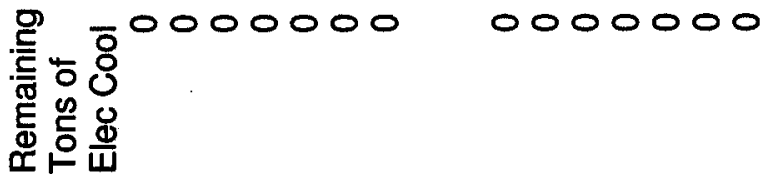

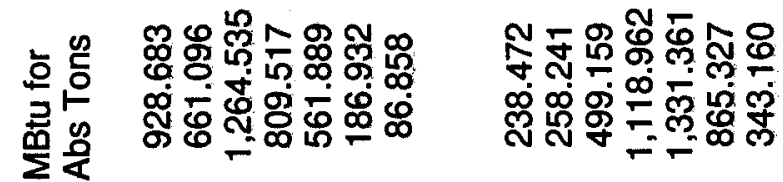

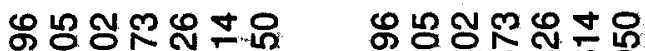

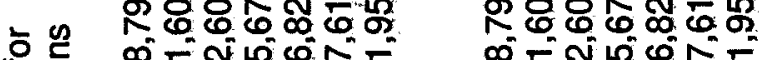

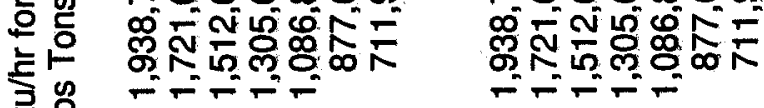

密巻

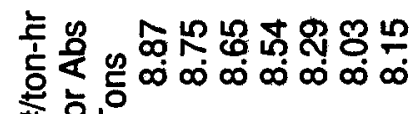

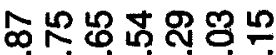

$\infty \infty \infty \infty \infty \infty$

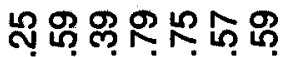

นึุ

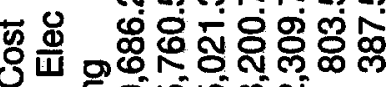

政的的

कृष

앙 웅

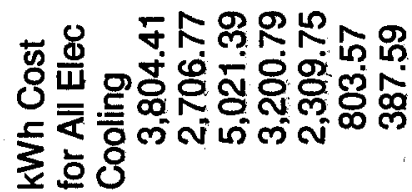

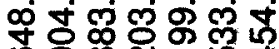

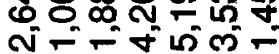

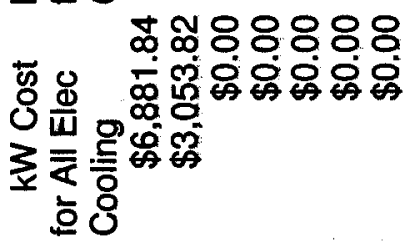

숴유윰ำ

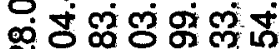

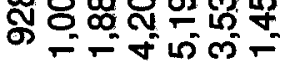

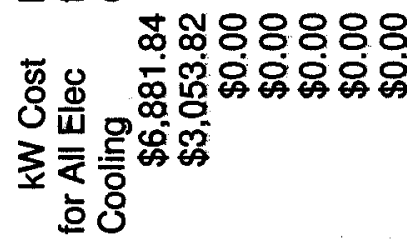

\%888888

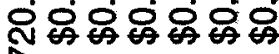

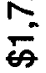

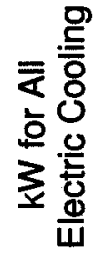

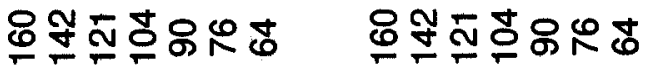

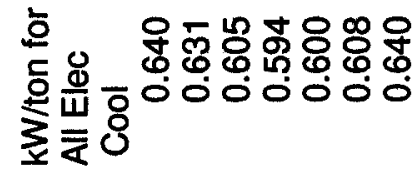

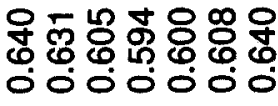



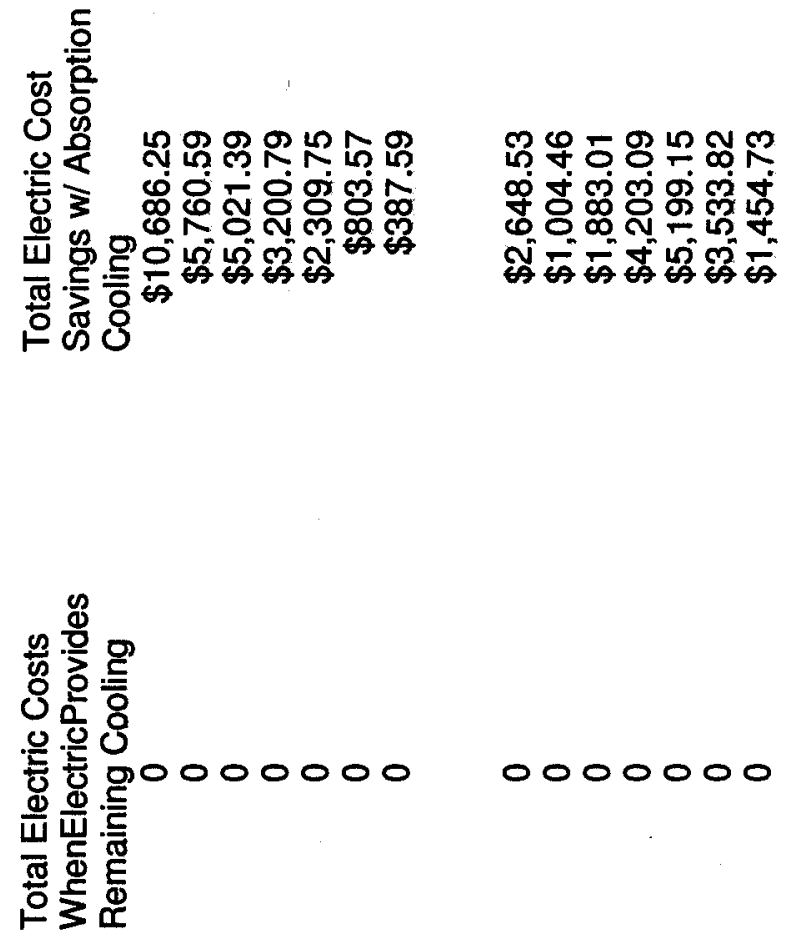

0000000

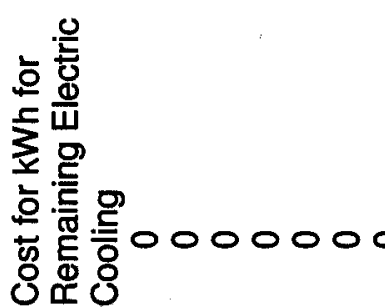

0000000

高:

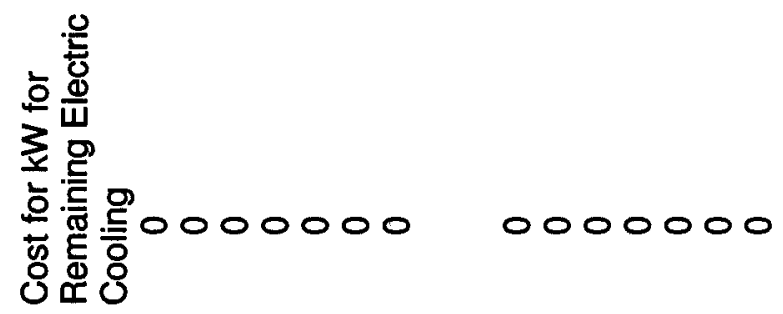

产宽0000000 0000000 主市声 


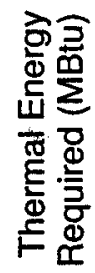

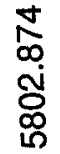

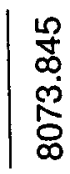
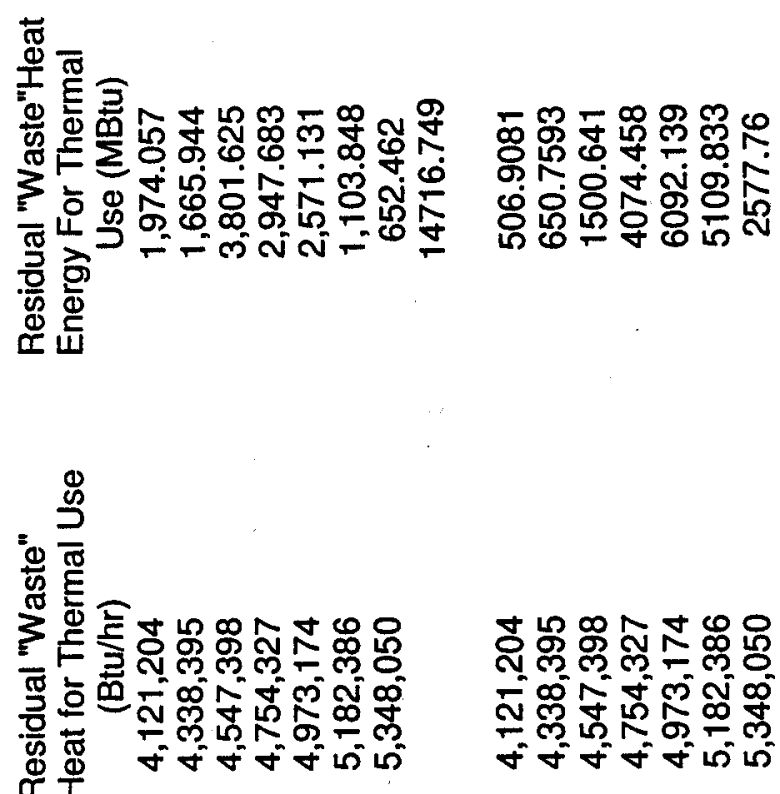

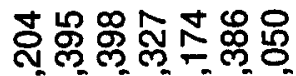
政

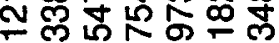

壬壬

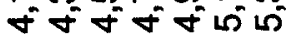

눈옳

후융

บํํำผ ำำ

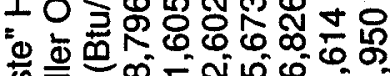

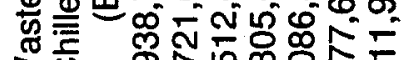

늉유유유유

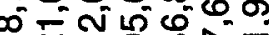

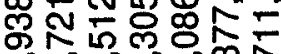
३U一 - = 吕

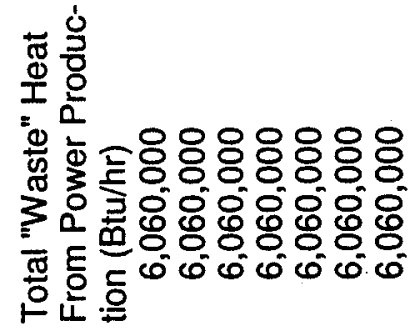

용융응용용용 $88088 \%$ 0 0 \% 


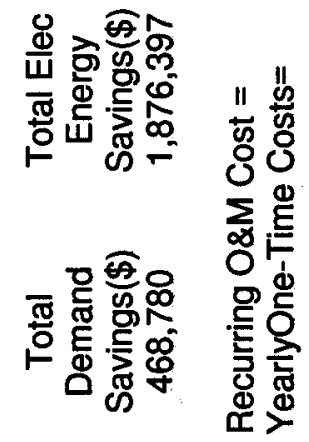

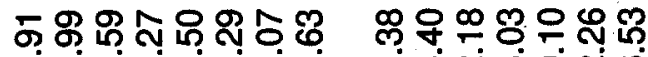

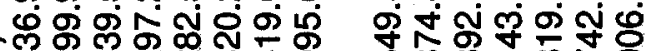

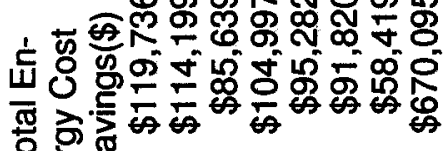

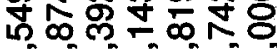

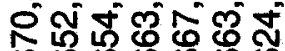

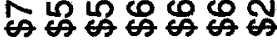
的
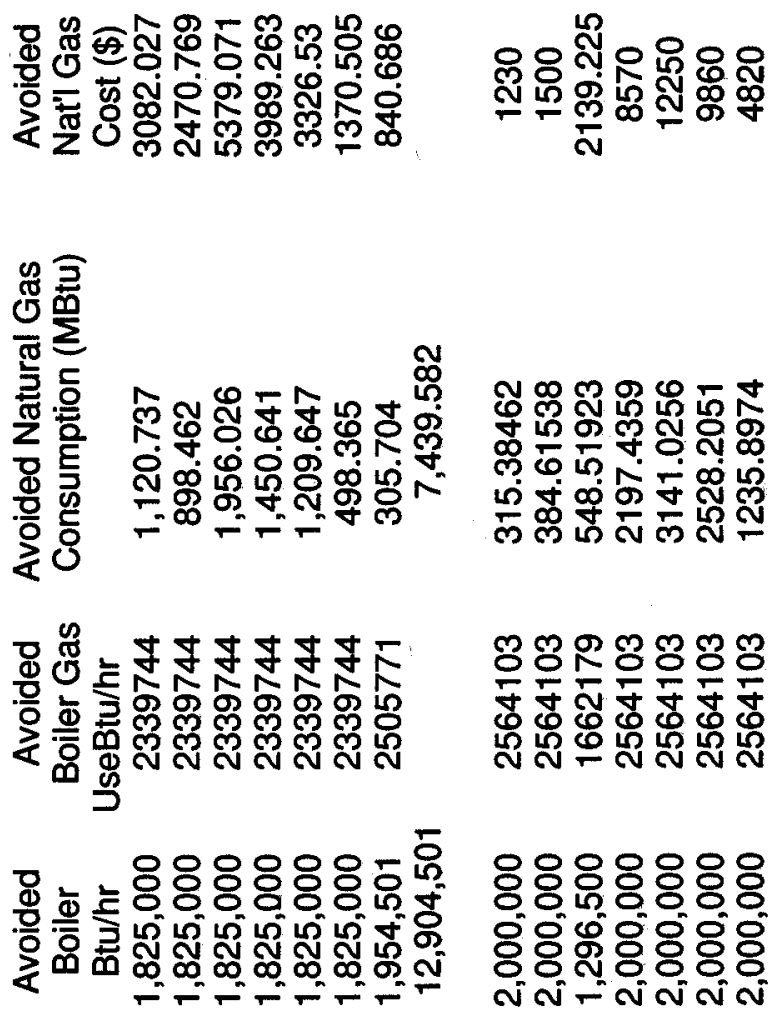


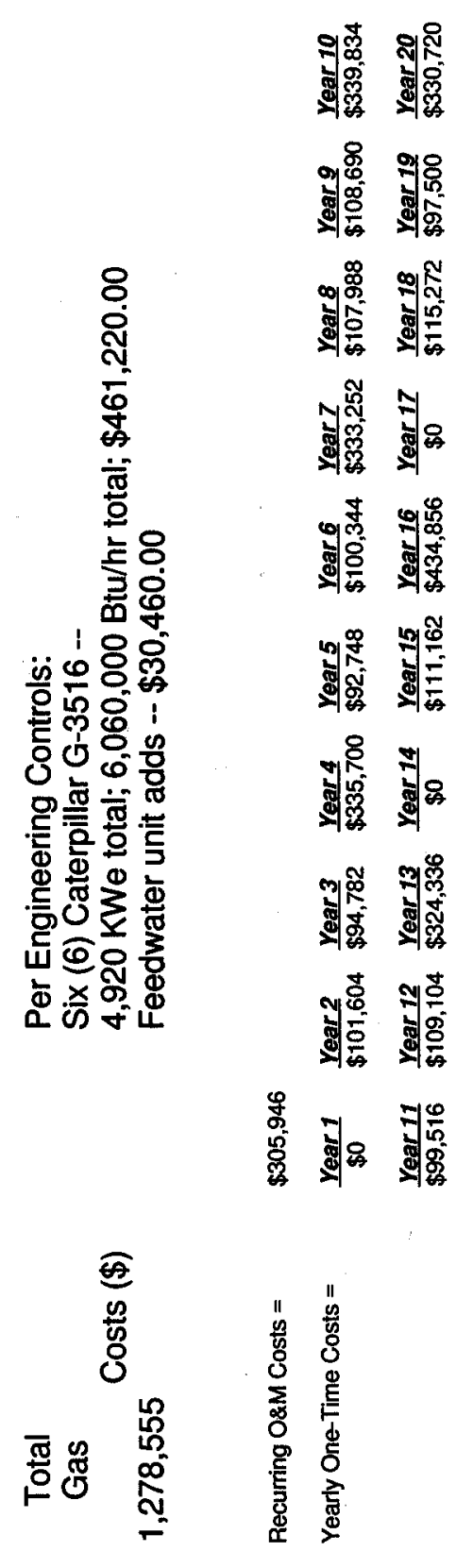




\title{
Appendix L: Calculated Paybacks and Savings-to-Investment Ratio for Option 1
}

\author{
Life Cycle Cost Analysis Study: Option \#1 \\ Energy Conservation Investment Program (ECIP) \\ Installation \& Location: Davis-Monthan AFB \\ Region data: ARIZONA Census Region: 4 \\ Project NO. \& Title: 250-Ton Chiller Replacement \\ Fiscal Year: 97 Discrete Portion: Option \#1 \\ Analysis Date: 10/01/97 Economic Life: 20 years \\ Prepared by: William $\mathrm{T}$ Brown III \\ LCCID FY96 \\ ECIP Summary Report
}

1. Investment
A. Construction Cost
$\$ 260,963$
B. $\mathrm{SIOH}$
$\$ 14,353$
C. Design Cost
D. Total Cost $(1 \mathrm{~A}+1 \mathrm{~B}+1 \mathrm{C})$
$\$ 15,658$
E. Salvage Value of Existing Equip.
$\$ 290,974$
F. Public Utility Company Rebate
$\$ 0$
G. Total Investment (1D-1E-1F)
$\$ 290,974$

2. Energy Savings (+) / Costs (-)

Date of NISTIR 85-3273-X used for Discount Factors Oct 1995

\begin{tabular}{|c|c|c|c|c|c|c|c|}
\hline Fuel & Price & $\begin{array}{l}\text { Price } \\
\text { Units }\end{array}$ & $\begin{array}{l}\text { Usage } \\
\text { Savings }\end{array}$ & $\begin{array}{l}\text { Usage } \\
\text { Units }\end{array}$ & $\begin{array}{r}\text { Annual } \\
\text { Savings }\end{array}$ & $\begin{array}{l}\text { Discount } \\
\text { Factor }\end{array}$ & $\begin{array}{l}\text { Discounted } \\
\text { Savings }\end{array}$ \\
\hline $\begin{array}{l}=== \\
\text { Elec }\end{array}$ & $======$ & $\begin{array}{l}===== \\
\text { /Mwatt }\end{array}$ & 792 & Mwatt- & $\begin{aligned}== & ====== \\
& \$ 36,441\end{aligned}$ & $\begin{array}{r}======= \\
14.47\end{array}$ & $=====$ \\
\hline Elec. Deman & & & & & & & \\
\hline $\begin{array}{l}\text { Natural Gas } \\
\text { TOTAL }\end{array}$ & $\$ 3.4$ & /Mbtus & $\begin{array}{l}-5,502 \\
-2,799\end{array}$ & $\begin{array}{l}\text { Mbtus } \\
\text { Mbtus }\end{array}$ & $\begin{array}{r}-\$ 18,706 \\
\$ 31,111\end{array}$ & 17.32 & $\begin{array}{r}-\$ 323,993 \\
\$ 383,491\end{array}$ \\
\hline
\end{tabular}

3. Non Energy Savings (+) / Costs (-)

\begin{tabular}{|c|c|c|c|c|}
\hline Item & $\begin{array}{c}\text { Savings / } \\
\text { Cost }\end{array}$ & Year & \begin{tabular}{|l} 
Discount \\
Factor
\end{tabular} & $\begin{array}{c}\text { Discounted } \\
\text { Savings/Cost }\end{array}$ \\
\hline ANNUAL TOTAL & $\$ 0$ & $= \pm==$ & & $\$ 0$ \\
\hline ONE TIME TOTAL & $\$ 0$ & & & $\$ 0$ \\
\hline TOTAL & $\$ 0$ & & & $\$ 0$ \\
\hline
\end{tabular}

4. First Year Dollar Savings

$\$ 31,111$

5. Simple Payback Period (Years)

6. Total Net Discounted Savings

7. Savings to Investment Ratio

$\$ 383,491$

If $<1$, Project does not qualify

8. Adjusted Internal Rate of Return 


\section{Appendix M: Calculated Paybacks and Savings-to-Investment Ratio for Option 2a}

\author{
Life Cycle Cost Analysis Study: Option \#2a \\ Energy Conservation Investment Program (ECIP) \\ Installation \& Location: Davis-Monthan AFB \\ Region data: ARIZONA Census Region: 4 \\ Project NO. \& Title: 250-Ton Chiller Replacement \\ Fiscal Year: 97 Discrete Portion: Option \#2a \\ Analysis Date: 10/01/97 Economic Life: 20 years \\ Prepared by: William T Brown III
}

ECIP Summary Report
LCCID FY96

1. Investment
A. Construction Cost
B. $\mathrm{SIOH}$
C. Design Cost
D. Total Cost $(1 \mathrm{~A}+1 \mathrm{~B}+1 \mathrm{C})$
E. Salvage Value of Existing Equip.
F. Public Utility Company Rebate
G. Total Investment (1D-1E-1F)

$$
\begin{array}{r}
\$ 176,011 \\
\$ 9,681 \\
\$ 10,561 \\
\$ 196,252 \\
\$ 0 \\
\$ 0 \\
\$ 196,252
\end{array}
$$

\begin{tabular}{|c|c|c|c|c|c|c|c|}
\hline $\begin{array}{c}\text { Fuel } \\
===========\end{array}$ & $\begin{array}{l}\text { Price } \\
======\end{array}$ & $\begin{array}{l}\text { Price } \\
\text { Units } \\
======\end{array}$ & $\begin{array}{l}\text { Usage } \\
\text { Savings } \\
=======\end{array}$ & $\begin{array}{l}\text { Usage } \\
\text { Units } \\
======\end{array}$ & $\begin{array}{c}\text { Annual } \\
\text { Savings } \\
========\end{array}$ & $\begin{array}{l}\text { Discount } \\
\text { Factor } \\
========\end{array}$ & $\begin{array}{l}\text { Discounted } \\
\text { Savings } \\
==========\end{array}$ \\
\hline $\begin{array}{l}\text { Electricity } \\
\text { Elec. Deman }\end{array}$ & $\$ 46$ & /Mwatt & 5,361 & Mwatt- & $\begin{array}{r}\$ 246,590 \\
\$ 45,670\end{array}$ & $\begin{array}{l}14.47 \\
13.47\end{array}$ & $\begin{array}{r}\$ 3,568,160 \\
\$ 615,175\end{array}$ \\
\hline $\begin{array}{l}\text { Natural Gas } \\
\text { TOTAL }\end{array}$ & $\$ 3.4$ & /Mbtus & $\begin{array}{l}-45,660 \\
-27,369\end{array}$ & $\begin{array}{l}\text { Mbtus } \\
\text { Mbtus }\end{array}$ & $\begin{array}{r}-\$ 155,244 \\
\$ 137,016\end{array}$ & 17.32 & $\begin{array}{r}-\$ 2,688,826 \\
\$ 1,494,509\end{array}$ \\
\hline
\end{tabular}

2. Energy Savings (+) / Costs (-) Date of NISTIR 85-3273-X used for Discount Factors Oct 1995 
3. Non Energy Savings (+) / Costs (-)

\begin{tabular}{|c|c|c|c|c|}
\hline 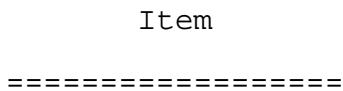 & $\begin{array}{l}\text { Savings/ } \\
\text { Cost } \\
========\end{array}$ & $\begin{array}{l}\text { Year } \\
=======\end{array}$ & $\begin{array}{l}\text { Discount } \\
\text { Factor } \\
========\end{array}$ & $\begin{array}{l}\text { Discounted } \\
\text { Savings / Cost } \\
===========\end{array}$ \\
\hline Baseline Maint & $-\$ 50,991$ & Annual & 13.47 & $-\$ 686,849$ \\
\hline ANNUAL TOTAL & $-\$ 50,991$ & & & $-\$ 686,849$ \\
\hline Maintenance & $-\$ 16,934$ & 2 & .92 & $-\$ 15,626$ \\
\hline Maintenance & $-\$ 15,797$ & 3 & .89 & $-\$ 14,003$ \\
\hline Maintenance & $-\$ 55,950$ & 4 & .85 & $-\$ 47,643$ \\
\hline Maintenance & $-\$ 15,458$ & 5 & .82 & $-\$ 12,644$ \\
\hline Maintenance & $-\$ 16,724$ & 6 & .79 & $-\$ 13,141$ \\
\hline Maintenance & $-\$ 55,542$ & 7 & .75 & $-\$ 41,924$ \\
\hline Maintenance & $-\$ 17,998$ & 8 & .73 & $-\$ 13,050$ \\
\hline itenance & 8,115 & 9 & .7 & $-\$ 12,618$ \\
\hline Maintenance & $-\$ 56,639$ & 10 & .67 & $-\$ 37,897$ \\
\hline Maintenance & $-\$ 16,586$ & 11 & .64 & $-\$ 10,661$ \\
\hline Maintenance & $-\$ 18,184$ & 12 & .62 & $-\$ 11,227$ \\
\hline Mai & $-\$ 5$ & 13 & .59 & $-\$ 32,062$ \\
\hline Maintenance & $-\$ 18,527$ & 15 & .55 & $-\$ 10,140$ \\
\hline Maintenance & $-\$ 72,476$ & 16 & .53 & $-\$ 38,105$ \\
\hline enance & $-\$ 19,212$ & 18 & .49 & $-\$ 9,321$ \\
\hline Maintenance & $-\$ 16,250$ & 19 & .47 & $-\$ 7,573$ \\
\hline Maintenance & $-\$ 55,120$ & 20 & .45 & $-\$ 24,677$ \\
\hline ONE TIME TOTAL & $-\$ 539,568$ & & & $-\$ 352,314$ \\
\hline TOTAL & $-\$ 590,559$ & & & $-\$ 1,039,163$ \\
\hline
\end{tabular}

Life Cycle Cost Analysis Study: DAVMON2.LC

Energy Conservation Investment Program (ECIP)

Installation \& Location: Davis-Monthan AFB

Region data: ARIZONA Census Region: 4

Project No. \& Title: 250-Ton Chiller Replacement

Fiscal Year: 97 Discrete Portion: Option \#2a

Analysis Date: 10/01/97 Economic Life: 20 years

Prepared by: William $\mathrm{T}$ Brown III

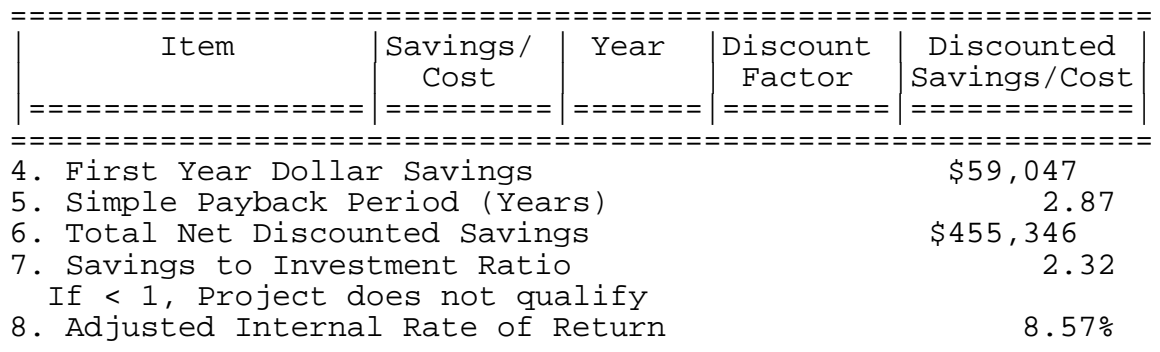




\section{Appendix N: Calculated Paybacks and Savings-to-Investment Ratio for Option 2b}

\author{
Life Cycle Cost Analysis Study: Option \#2b \\ Energy Conservation Investment Program (ECIP) \\ Installation \& Location: Davis-Monthan AFB \\ Region data: ARIZONA Census Region: 4 \\ Project NO. \& Title: 250-Ton Chiller Replacement \\ Fiscal Year: 97 Discrete Portion: Option \#2b \\ Analysis Date: 10/01/97 Economic Life: 20 years \\ Prepared by: William $\mathrm{T}$ Brown III
}

ECIP Summary Report
LCCID FY96
A. Construction Cost
$\$ 211,132$
B. $\mathrm{SIOH}$
C. Design Cost
D. Total Cost $(1 \mathrm{~A}+1 \mathrm{~B}+1 \mathrm{C})$
E. Salvage Value of Existing Equip.
F. Public Utility Company Rebate
$\$ 11,612$
$\$ 12,668$
$\$ 235,412$
G. Total Investment (1D-1E-1F)

2. Energy Savings (+) / Costs (-) Date of NISTIR 85-3273-X used for Discount Factors Oct 1995

\begin{tabular}{|c|c|c|c|c|c|c|c|}
\hline Fuel & Price & $\begin{array}{l}\text { Price } \\
\text { Units }\end{array}$ & $\begin{array}{l}\text { Usage } \\
\text { Savings }\end{array}$ & $\begin{array}{l}\text { Usage } \\
\text { Units }\end{array}$ & $\begin{array}{r}\text { Annual } \\
\text { Savings }\end{array}$ & $\begin{array}{l}\text { Discount } \\
\text { Factor }\end{array}$ & $\begin{array}{l}\text { Discounted } \\
\text { Savings }\end{array}$ \\
\hline Electricity & $\$ 46$ & /Mwatt & 7,188 & Mwatt- & $\$ 330,671$ & 14.47 & $\$ 4,784,803$ \\
\hline $\begin{array}{l}\text { NLec. Deman } \\
\text { Natural Gas } \\
\text { TOTAL }\end{array}$ & $\$ 3.4$ & /Mbtus & $\begin{array}{l}-63,192 \\
-38,664\end{array}$ & $\begin{array}{l}\text { Mbtus } \\
\text { Mbtus }\end{array}$ & $\begin{array}{r}\$ 46,064 \\
-\$ 214,852 \\
\$ 162,483\end{array}$ & $\begin{array}{l}13.41 \\
17.32\end{array}$ & $\begin{array}{r}-\$ 3,721,231 \\
\$ 1,692,136\end{array}$ \\
\hline
\end{tabular}


3. Non Energy Savings (+) / Costs (-)

\begin{tabular}{|c|c|c|c|c|}
\hline 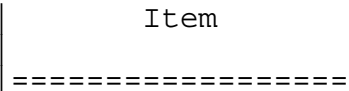 & $\begin{array}{l}\text { Savings / } \\
\text { Cost } \\
========\end{array}$ & $\begin{array}{l}\text { Year } \\
=======\end{array}$ & $\begin{array}{l}\text { Discount } \\
\text { Factor } \\
=========\end{array}$ & $\begin{array}{l}\text { Discounted } \\
\text { Savings/Cost } \\
===========\end{array}$ \\
\hline Baseline Maint & $-\$ 50,991$ & Annual & 13.47 & $-\$ 686,849$ \\
\hline ANNUAL TOTAL & $-\$ 50,991$ & & & $-\$ 686,849$ \\
\hline Maintenance & $-\$ 16,934$ & 2 & .92 & $-\$ 15,626$ \\
\hline Maintenance & $-\$ 15,797$ & 3 & .89 & $-\$ 14,003$ \\
\hline Maintenance & $-\$ 55,950$ & 4 & .85 & $-\$ 47,643$ \\
\hline Maintenance & $-\$ 15,458$ & 5 & .82 & $-\$ 12,644$ \\
\hline Maintenance & $-\$ 16,724$ & 6 & .79 & $-\$ 13,141$ \\
\hline Maintenance & $-\$ 55,542$ & 7 & .75 & $-\$ 41,924$ \\
\hline Maintenance & $-\$ 17,998$ & 8 & .73 & $-\$ 13,050$ \\
\hline Maintenance & $-\$ 18,115$ & 9 & .7 & $-\$ 12,618$ \\
\hline Maintenance & $-\$ 56,639$ & 10 & .67 & $-\$ 37,897$ \\
\hline Maintenance & $-\$ 16,586$ & 11 & .64 & $-\$ 10,661$ \\
\hline Maintenance & $-\$ 18,184$ & 12 & .62 & $-\$ 11,227$ \\
\hline Main & $-\$ 54,056$ & 13 & .59 & $-\$ 32,062$ \\
\hline Maintenance & $-\$ 18,527$ & 15 & .55 & $-\$ 10,140$ \\
\hline Maintenance & $-\$ 72,476$ & 16 & .53 & $-\$ 38,105$ \\
\hline Maintenance & $-\$ 19,212$ & 18 & .49 & $-\$ 9,321$ \\
\hline Maintenance & $-\$ 16,250$ & 19 & .47 & $-\$ 7,573$ \\
\hline Maintenance & $-\$ 55,120$ & 20 & .45 & $-\$ 24,677$ \\
\hline ONE TIME TOTAL & $-\$ 539,568$ & & & $-\$ 352,314$ \\
\hline TOTAL & $\$ 590,559$ & & & $-\$ 1,039,163$ \\
\hline
\end{tabular}

Life Cycle Cost Analysis Study: DAVMON3.LC

Energy Conservation Investment Program (ECIP)

Installation \& Location: Davis-Monthan AFB

Region data: ARIZONA Census Region: 4

Project No. \& Title: 250-Ton Chiller Replacement

Fiscal Year: 97 Discrete Portion: Option \#2b

Analysis Date: 10/01/97 Economic Life: 20 years

Prepared by: William $\mathrm{T}$ Brown III

\begin{tabular}{|c|c|c|c|c|}
\hline$================$ & $\begin{array}{l}\text { Savings } \\
\text { Cost } \\
========\end{array}$ & $=======$ & $\begin{array}{l}\text { Discount } \\
\text { Factor } \\
========\end{array}$ & $\begin{array}{c}\text { Discounted } \\
\text { Savings/Cost } \\
===========\end{array}$ \\
\hline \multicolumn{5}{|c|}{ =" =" } \\
\hline \multicolumn{4}{|c|}{ 4. First Year Dollar Savings } & $\$ 84,513$ \\
\hline \multicolumn{4}{|c|}{ 5. Simple Payback Period (Years) } & 2.51 \\
\hline \multirow{2}{*}{\multicolumn{4}{|c|}{$\begin{array}{l}\text { 6. Total Net Discounted Savings } \\
\text { 7. Savings to Investment Ratio }\end{array}$}} & $\$ 652,973$ \\
\hline \multicolumn{2}{|c|}{ If $<1$, Project does not qualify } & & & 2.77 \\
\hline $\begin{array}{l}\text { It } 1, \text { Project de } \\
\text {. Adjusted Internal }\end{array}$ & $\begin{array}{l}\text { oes not qua } \\
\text { I Rate of } \mathrm{F}\end{array}$ & $\begin{array}{l}\text { allty } \\
\text { Return }\end{array}$ & & $9.55 \%$ \\
\hline
\end{tabular}




\section{Appendix 0: Calculated Paybacks and Savings-to-Investment Ratio for Option 2c}

\author{
Life Cycle Cost Analysis Study: Option \#2C \\ Energy Conservation Investment Program (ECIP) \\ Installation \& Location: Davis-Monthan AFB \\ Region data: ARIZONA Census Region: 4 \\ Project NO. \& Title: 250-Ton Chiller Replacement \\ Fiscal Year: 97 Discrete Portion: Option \#2c \\ Analysis Date: 10/01/97 Economic Life: 20 years \\ Prepared by: William $\mathrm{T}$ Brown III
}

ECIP Summary Report
LCCID FY96
A. Construction Cost
$\$ 427,380$
B. $\mathrm{SIOH}$
C. Design Cost
D. Total Cost $(1 \mathrm{~A}+1 \mathrm{~B}+1 \mathrm{C})$
E. Salvage Value of Existing Equip.
F. Public Utility Company Rebate
$\$ 23,506$
$\$ 25,643$
$\$ 476,529$
G. Total Investment (1D-1E-1F)

2. Energy Savings (+) / Costs (-) Date of NISTIR 85-3273-X used for Discount Factors Oct 1995

\begin{tabular}{|c|c|c|c|c|c|c|c|}
\hline Fuel & Price & $\begin{array}{l}\text { Price } \\
\text { Units }\end{array}$ & $\begin{array}{l}\text { Usage } \\
\text { Savings }\end{array}$ & $\begin{array}{l}\text { Usage } \\
\text { Units }\end{array}$ & $\begin{array}{r}\text { Annual } \\
\text { Savings }\end{array}$ & $\begin{array}{l}\text { Discount } \\
\text { Factor }\end{array}$ & $\begin{array}{c}\text { Discounted } \\
\text { Savings }\end{array}$ \\
\hline Electricity & $\$ 46$ & /Mwatt & 14,289 & Mwatt- & $\$ 657,281$ & 14.47 & $\$ 9,510,858$ \\
\hline $\begin{array}{l}\text { Elec. Deman } \\
\text { Natural Gas } \\
\text { TOTAL }\end{array}$ & $\$ 3.4$ & /Mbtus & $\begin{array}{r}-113,421 \\
-64,666\end{array}$ & $\begin{array}{l}\text { Mbtus } \\
\text { Mbtus }\end{array}$ & $\begin{array}{r}\$ 154,883 \\
-\$ 385,633 \\
\$ 426,531\end{array}$ & $\begin{array}{l}13.47 \\
17.32\end{array}$ & $\begin{array}{r}\$ 2,086,274 \\
-\$ 6,679,160 \\
\$ 4,917,973\end{array}$ \\
\hline
\end{tabular}


3. Non Energy Savings (+) / Costs (-)

\begin{tabular}{|c|c|c|c|c|}
\hline Item & $\begin{array}{l}\text { Savings / } \\
\text { Cost }\end{array}$ & Year & $\begin{array}{l}\text { Discount } \\
\text { Factor }\end{array}$ & $\begin{array}{l}\text { Discounted } \\
\text { Savings/Cost }\end{array}$ \\
\hline $\begin{array}{l}================ \\
\text { Baseline Maint }\end{array}$ & $\begin{array}{l}======== \\
-\$ 101982\end{array}$ & $\begin{array}{l}====== \\
\text { Annual }\end{array}$ & $\begin{array}{r}======= \\
13.47\end{array}$ & $\begin{array}{r}========== \\
-\$ 1,373,698\end{array}$ \\
\hline ANNUAL TOTAL & $-\$ 101,982$ & Anllud 1 & & $-\$ 1,373,698$ \\
\hline Maintenance & $-\$ 33,868$ & 2 & .92 & $-\$ 31,253$ \\
\hline Maintenance & $-\$ 31,594$ & 3 & .89 & $-\$ 28,006$ \\
\hline Maintenance & $-\$ 111,900$ & 4 & .85 & $-\$ 95,286$ \\
\hline Maintenance & $-\$ 30,916$ & 5 & .82 & $-\$ 25,289$ \\
\hline Maintenance & $-\$ 33,448$ & 6 & .79 & $-\$ 26,282$ \\
\hline Maintenance & $-\$ 111,084$ & 7 & .75 & $-\$ 83,849$ \\
\hline Maintenance & $-\$ 35,996$ & 8 & .73 & $-\$ 26,100$ \\
\hline Maintenance & $-\$ 36,230$ & 9 & .7 & $-\$ 25,235$ \\
\hline Maintenance & 3,278 & 10 & .67 & $-\$ 75,795$ \\
\hline Maintenance & $-\$ 33,172$ & 11 & .64 & $-\$ 21,321$ \\
\hline Maintenance & 368 & 12 & .62 & $-\$ 22,455$ \\
\hline Maintenance & $-\$ 108,112$ & 13 & .59 & $-\$ 64,123$ \\
\hline Maintenance & $-\$ 37,054$ & 15 & .55 & $-\$ 20,280$ \\
\hline Maintenance &, 952 & 16 & .53 & $-\$ 76,210$ \\
\hline Maintenance & 3,424 & 18 & .49 & $-\$ 18,642$ \\
\hline Maintenance & 500 & 19 & .47 & $-\$ 15,147$ \\
\hline Maintenance & $-\$ 110,240$ & 20 & .45 & $-\$ 49,354$ \\
\hline ONE TIME TOTAI & $-\$ 1,079,1$ & & & $-\$ 704,628$ \\
\hline TOTAL & $-\$ 1,181$ & & & $\$ 2,078,32$ \\
\hline
\end{tabular}

Life Cycle Cost Analysis Study: DAVMON4.LC

Energy Conservation Investment Program (ECIP)

Installation \& Location: Davis-Monthan AFB

Region data: ARIZONA Census Region: 4

Project NO. \& Title: 250-Ton Chiller Replacement

Fiscal Year: 97 Discrete Portion: Option \#2c

Analysis Date: 10/01/97 Economic Life: 20 years

Prepared by: William $\mathrm{T}$ Brown III

\begin{tabular}{|c|c|c|c|c|}
\hline $\begin{array}{c}\text { Item } \\
=================\end{array}$ & $\begin{array}{l}\text { Savings / } \\
\text { Cost } \\
========\end{array}$ & $=======$ & $\begin{array}{l}\text { Discount } \\
\text { Factor } \\
========\end{array}$ & $=\begin{array}{l}\text { Discounted } \\
\text { Savings /Cost } \\
===========\end{array}$ \\
\hline \multicolumn{5}{|c|}{ =" =" =" } \\
\hline \multicolumn{5}{|c|}{ 4. First Year Dollar Savings } \\
\hline \multicolumn{5}{|c|}{ 5. Simple Payback Period (Years) } \\
\hline \multicolumn{5}{|c|}{$\begin{array}{l}\text { 6. Total Net Discounted Savings } \\
\text { 7. Savings to Investment Ratio }\end{array}$} \\
\hline \multirow{2}{*}{\multicolumn{5}{|c|}{$\begin{array}{l}\text { 7. Savings to Investment Ratio } \\
\text { If }<1 \text {, Project does not qualify }\end{array}$}} \\
\hline & & & & $13.82 \%$ \\
\hline
\end{tabular}




\title{
Appendix P: Calculated Paybacks and Savings-to-Investment Ratio for Option 2d
}

\author{
Life Cycle Cost Analysis Study: Option \#2d \\ Energy Conservation Investment Program (ECIP) \\ Installation \& Location: Davis-Monthan AFB \\ Region data: ARIZONA Census Region: 4 \\ Project NO. \& Title: 250-Ton Chiller Replacement \\ Fiscal Year: 97 Discrete Portion: Option \#2d \\ Analysis Date: 10/01/97 Economic Life: 20 years \\ Prepared by: William $\mathrm{T}$ Brown III \\ 1. Investment \\ ECIP Summary Report
A. Construction Cost
$\$ 573,172$
B. $\mathrm{SIOH}$
C. Design Cost
D. Total Cost $(1 \mathrm{~A}+1 \mathrm{~B}+1 \mathrm{C})$
$\$ 31,524$
E. Salvage Value of Existing Equip.
639,087
- Public Utility Company Rebate
$\$ 0$
G. Total Investment (1D-1E-1F)
$\$ 639,087$

LCCID FY96

2. Energy Savings (+) / Costs (-)

Date of NISTIR 85-3273-X used for Discount Factors Oct 1995

\begin{tabular}{|c|c|c|c|c|c|c|c|}
\hline Fuel & Price & $\begin{array}{l}\text { Price } \\
\text { Units }\end{array}$ & $\begin{array}{l}\text { Usage } \\
\text { Savings }\end{array}$ & $\begin{array}{l}\text { Usage } \\
\text { Units }\end{array}$ & $\begin{array}{r}\text { Annual } \\
\text { Savings }\end{array}$ & $\begin{array}{l}\text { Discount } \\
\text { Factor }\end{array}$ & $\begin{array}{l}\text { Discounted } \\
\text { Savings }\end{array}$ \\
\hline & $\$ 46$ & $=====$ & $=======$ & Mratt & 5.1 & & \\
\hline Elec. Deman & & & 20,340 & & $\$ 2$ & & \\
\hline $\begin{array}{l}\text { Natural Gas } \\
\text { TOTAL }\end{array}$ & $\$ 3.4$ & /Mbtus & $\begin{array}{l}-178,587 \\
-108,480\end{array}$ & $\begin{array}{l}\text { Mbtus } \\
\text { Mbtus }\end{array}$ & $\begin{array}{r}-\$ 607,197 \\
\$ 569,995\end{array}$ & 17.32 & $\begin{array}{r}-\$ 10,516,65 \\
\$ 6,285,267\end{array}$ \\
\hline
\end{tabular}


3. Non Energy Savings (+) / Costs (-)

\begin{tabular}{|c|c|c|c|c|}
\hline 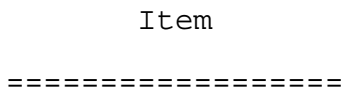 & $\begin{array}{l}\text { Savings / } \\
\text { Cost } \\
========\end{array}$ & $\begin{array}{l}\text { Year } \\
=======\end{array}$ & $\begin{array}{l}\text { Discount } \\
\text { Factor } \\
========\end{array}$ & $\begin{array}{l}\text { Discounted } \\
\text { Savings/Cost } \\
==========\end{array}$ \\
\hline Baseline Maint & $-\$ 152,973$ & Annual & 13.47 & $-\$ 2,060,546$ \\
\hline ANNUAL TOTAL & $-\$ 152,973$ & & & $-\$ 2,060$ \\
\hline Maintenance & $-\$ 50,802$ & 2 & .92 & $-\$ 46,879$ \\
\hline Eenance & $-\$ 47,391$ & 3 & .89 & $-\$ 42,009$ \\
\hline Maintenance & $-\$ 167,840$ & 4 & .85 & $-\$ 142,920$ \\
\hline Maintenance & $-\$ 46,374$ & 5 & .82 & $-\$ 37,933$ \\
\hline Maintenance & $-\$ 50,172$ & 6 & .79 & $-\$ 39,424$ \\
\hline Maintenance & $-\$ 166,626$ & 7 & .75 & $-\$ 125,773$ \\
\hline enance & $-\$ 53,994$ & 8 & .73 & $-\$ 39,151$ \\
\hline enance &, 345 & 9 & .7 & $-\$ 37,853$ \\
\hline Maintenance & $-\$ 169,917$ & 10 & .67 & $-\$ 113,692$ \\
\hline Maintenance & $-\$ 49,758$ & 11 & .64 & $-\$ 31,982$ \\
\hline Maintenance &, 552 & 12 & .62 & $-\$ 33,682$ \\
\hline tenance & $-\$ 16$ & 13 & .59 & $-\$ 96,185$ \\
\hline Maintenance & $-\$ 5$ & 1 & .55 & $-\$ 30,420$ \\
\hline Maintenance & $-\$ 217,428$ & 16 & .53 & $-\$ 114,315$ \\
\hline Maintenance & $-\$ 57,636$ & 18 & .49 & $-\$ 27,963$ \\
\hline Maintenance & $-\$ 48,750$ & 19 & .47 & $-\$ 22,720$ \\
\hline Maintenance & $-\$ 165,360$ & 20 & .45 & $-\$ 74,031$ \\
\hline TIME TOTAL & $-\$ 1,618,6$ & & & $-\$ 1,056,933$ \\
\hline TOTAL & $-\$ 1,771,6$ & & & $-\$ 3,117,479$ \\
\hline
\end{tabular}

Life Cycle Cost Analysis Study: DAVMON5.LC

Energy Conservation Investment Program (ECIP)

LCCID FY96

Installation \& Location: Davis-Monthan AFB

Region data: ARIZONA Census Region: 4

Project NO. \& Title: 250-Ton Chiller Replacement

Fiscal Year: 97 Discrete Portion: Option \#2d

Analysis Date: 10/01/97 Economic Life: 20 years

Prepared by: William $\mathrm{T}$ Brown III

\begin{tabular}{|c|c|c|c|c|}
\hline$==================$ & $\begin{array}{l}\text { Savings } \\
\text { Cost } \\
========\end{array}$ & $=======$ & $\begin{array}{l}\text { Discount } \\
\text { Factor } \\
========\end{array}$ & $=\mid \begin{array}{c}\text { Discounted } \\
\text { Savings } / \text { Cost } \\
===========\end{array}$ \\
\hline \multicolumn{5}{|c|}{ 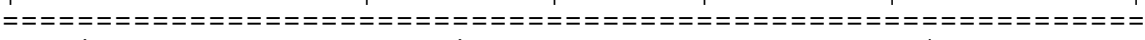 } \\
\hline \multicolumn{4}{|c|}{ 4. First Year Dollar Savings } & $\$ 336,088$ \\
\hline \multicolumn{4}{|c|}{ 5. Simple Payback Period (Years) } & 1.75 \\
\hline \multirow{2}{*}{\multicolumn{4}{|c|}{$\begin{array}{l}\text { 6. Total Net Discounted Savings } \\
\text { 7. Savings to Investment Ratio }\end{array}$}} & $\$ 3,167,788$ \\
\hline & & & & \\
\hline $\begin{array}{l}\text { It } 1 \text {, Project de } \\
\text { - Adusted Internal }\end{array}$ & $\begin{array}{l}\text { oes not qua } \\
\text { I Rate of } \mathrm{F}\end{array}$ & $\begin{array}{l}\text { alify } \\
\text { Return }\end{array}$ & & $12.77 \%$ \\
\hline
\end{tabular}




\section{Appendix Q: Calculated Paybacks and Savings-to-Investment Ratio for Option 3a}

\begin{abstract}
Life Cycle Cost Analysis Study: Option \#3a Energy Conservation Investment Program (ECIP)

Installation \& Location: Davis-Monthan AFB Region data: ARIZONA Census Region: 4 Project NO. \& Title: 250-Ton Chiller Replacement Fiscal Year: 97 Discrete Portion: Option \#3a Analysis Date: 10/01/97 Economic Life: 20 years Prepared by: William $\mathrm{T}$ Brown III
\end{abstract}

ECIP Summary Report
A. Construction Cost
$\$ 241,710$
B. $\mathrm{SIOH}$
C. Design Cost
D. Total Cost $(1 \mathrm{~A}+1 \mathrm{~B}+1 \mathrm{C})$
E. Salvage Value of Existing Equip.
$\$ 13,294$
$\$ 14,503$
$\$ 269,507$
F. Public Utility Company Rebate
$\$ 0$
$\$ 0$
G. Total Investment (1D-1E-1F)
$\$ 269,507$

2. Energy Savings (+) / Costs (-) Date of NISTIR 85-3273-X used for Discount Factors Oct 1995

\begin{tabular}{|c|c|c|c|c|c|c|c|}
\hline Fuel & Price & $\begin{array}{l}\text { Price } \\
\text { Units } \\
=====\end{array}$ & $\begin{array}{l}\text { Usage } \\
\text { Savings } \\
======\end{array}$ & $\begin{array}{l}\text { Usage } \\
\text { Units } \\
======\end{array}$ & $\begin{array}{c}\text { Annual } \\
\text { Savings } \\
========\end{array}$ & $\begin{array}{l}\text { Discount } \\
\text { Factor } \\
=======\end{array}$ & $\begin{array}{l}\text { Discounted } \\
\text { Savings } \\
=========\end{array}$ \\
\hline tricity & $\$ 46$ & /Mwatt & 13,937 & Mwatt- & $\$ 641,0$ & & \\
\hline $\begin{array}{l}\text { Natural Gas } \\
\text { TOTAL }\end{array}$ & $\$ 3.4$ & /Mbtus & $\begin{array}{r}-113,421 \\
-65,867\end{array}$ & $\begin{array}{l}\text { Mbtus } \\
\text { Mbtus }\end{array}$ & $\begin{array}{r}-\$ 385,632 \\
\$ 402,834\end{array}$ & $\begin{array}{l}13.41 \\
17.32\end{array}$ & $\begin{array}{r}\$ 1,679,142 \\
-\$ 6,682,585\end{array}$ \\
\hline
\end{tabular}


3. Non Energy Savings (+) / Costs (-)

\begin{tabular}{|c|c|c|c|c|}
\hline 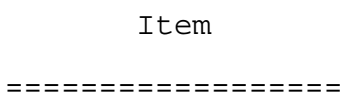 & $\begin{array}{l}\text { Savings / } \\
\text { Cost } \\
========\end{array}$ & $=======$ & $\begin{array}{l}\text { Discount } \\
\text { Factor } \\
=======\end{array}$ & $\begin{array}{l}\text { Discounted } \\
\text { Savings/Cost } \\
==========\end{array}$ \\
\hline Baseline Maint & $-\$ 101,982$ & Annual & 13.47 & $-\$ 1,373,698$ \\
\hline AL TOTAL & $-\$ 101,982$ & & & $-\$ 1,373$, \\
\hline Maintenance & $-\$ 33,868$ & 2 & .92 & $-\$ 31,253$ \\
\hline Maintenance & $-\$ 31,594$ & 3 & .89 & $-\$ 28,006$ \\
\hline Maintenance & $-\$ 111,900$ & 4 & .85 & $-\$ 95,286$ \\
\hline Maintenance & $-\$ 30,916$ & 5 & .82 & $-\$ 25,289$ \\
\hline Maintenance & $-\$ 33,448$ & 6 & .79 & $-\$ 26,282$ \\
\hline tenance & $-\$ 111,084$ & 7 & .75 & $-\$ 83,849$ \\
\hline nance & $-\$ 35,996$ & 8 & .73 & $-\$ 26,100$ \\
\hline Maintenance & $-\$ 36,230$ & 9 & .7 & $-\$ 25,235$ \\
\hline Maintenance & $-\$ 113,278$ & 10 & .67 & $-\$ 75,795$ \\
\hline Maintenance & $-\$ 33,172$ & 11 & .64 & $-\$ 21,321$ \\
\hline Maintenance & $-\$ 36,368$ & 12 & .62 & $-\$ 22,455$ \\
\hline Maintenance & $-\$ 108,112$ & 13 & .59 & $-\$ 64,123$ \\
\hline Maintenance & $-\$ 37,054$ & 15 & .55 & $-\$ 20,280$ \\
\hline Maintenance & $-\$ 144$ & 1 & .53 & $-\$ 76,2$ \\
\hline Maintenance & $-\$ 38,424$ & 18 & .49 & $-\$ 18,642$ \\
\hline Maintenance & $-\$ 32,500$ & 19 & .47 & $-\$ 15,147$ \\
\hline Maintenance & $-\$ 110,240$ & 20 & .45 & $-\$ 49,354$ \\
\hline ONE TIME TOTAL & $-\$ 1,079,1$ & & & $-\$ 704,628$ \\
\hline TOTAL & $-\$ 1,181,1$ & & & $-\$ 2,078,325$ \\
\hline
\end{tabular}

Life Cycle Cost Analysis Study: DAVMON6.LC

Energy Conservation Investment Program (ECIP)

Installation \& Location: Davis-Monthan AFB

Region data: ARIZONA Census Region: 4

Project NO. \& Title: 250-Ton Chiller Replacement

Fiscal Year: 97 Discrete Portion: Option \#3a

Analysis Date: 10/01/97 Economic Life: 20 years

Prepared by: William $\mathrm{T}$ Brown III

\begin{tabular}{|c|c|c|c|c|}
\hline 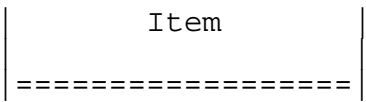 & \begin{tabular}{|} 
Savings $/$ \\
Cost \\
$========$
\end{tabular} & $=======$ & $\begin{array}{l}\text { Discount } \\
\text { Factor } \\
========\end{array}$ & $=\begin{array}{c}\text { Discounted } \\
\text { Savings/Cost } \\
===========\end{array}$ \\
\hline \multicolumn{5}{|c|}{ 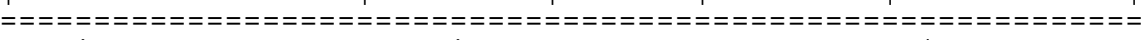 } \\
\hline \multicolumn{4}{|c|}{ 4. First Year Dollar Savings } & $\$ 246,895$ \\
\hline \multicolumn{4}{|c|}{ 5. Simple Payback Period (Years) } & 1.01 \\
\hline \multirow{2}{*}{\multicolumn{4}{|c|}{$\begin{array}{l}\text { 6. Total Net Discounted Savings } \\
\text { 7. Savings to Investment Ratio }\end{array}$}} & $\$ 2,504,260$ \\
\hline & & & & 9.29 \\
\hline \multicolumn{4}{|c|}{ If $<1$, Project does not qualify } & 16.37 \\
\hline
\end{tabular}




\section{Appendix R: Calculated Paybacks and Savings-to-Investment Ratio for Option 3b}

Life Cycle Cost Analysis Study: Option \#3b Energy Conservation Investment Program (ECIP)

Installation \& Location: Davis-Monthan AFB Region data: ARIZONA Census Region: 4 Project NO. \& Title: 250-Ton Chiller Replacement Fiscal Year: 97 Discrete Portion: Option \#3b Analysis Date: 10/01/97 Economic Life: 20 years Prepared by: William $\mathrm{T}$ Brown III

ECIP Summary Report
LCCID FY96
A. Construction Cost
$\$ 481,450$
B. $\mathrm{SIOH}$
C. Design Cost
D. Total Cost $(1 \mathrm{~A}+1 \mathrm{~B}+1 \mathrm{C})$
$\$ 26,480$
$\$ 28,887$
E. Salvage Value of Existing Equip.
$\$ 536,817$
F. Public Utility Company Rebate
$\$ 0$
G. Total Investment (1D-1E-1F)
$\$ 536,817$

2. Energy Savings (+) / Costs (-) Date of NISTIR 85-3273-X used for Discount Factors Oct 1995

\begin{tabular}{|c|c|c|c|c|c|c|c|}
\hline Fuel & Price & $\begin{array}{l}\text { Price } \\
\text { Units } \\
=====\end{array}$ & $\begin{array}{l}\text { Usage } \\
\text { Savings } \\
======\end{array}$ & $\begin{array}{l}\text { Usage } \\
\text { Units } \\
======\end{array}$ & $\begin{array}{c}\text { Annual } \\
\text { Savings } \\
========\end{array}$ & $\begin{array}{l}\text { Discount } \\
\text { Factor } \\
=======\end{array}$ & $\begin{array}{l}\text { Discounted } \\
\text { Savings } \\
=========\end{array}$ \\
\hline tricity & $\$ 46$. & /Mwatt & 26,943 & Mwatt- & $\$ 1,239,367$ & & $\$ 17$ \\
\hline $\begin{array}{l}\text { Natural Gas } \\
\text { TOTAL }\end{array}$ & $\$ 3.4$ & /Mbtus & $\begin{array}{l}-244,743 \\
-152,811\end{array}$ & $\begin{array}{l}\text { Mbtus } \\
\text { Mbtus }\end{array}$ & $\begin{array}{r}-\$ 832,128 \\
\$ 717,692\end{array}$ & 17.32 & $\begin{array}{r}-\$ 14,412,45 \\
\$ 7,702,992\end{array}$ \\
\hline
\end{tabular}


3. Non Energy Savings (+) / Costs (-)

\begin{tabular}{|c|c|c|c|c|}
\hline 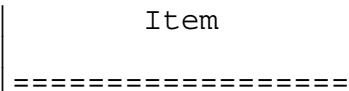 & $\begin{array}{l}\text { Savings / } \\
\text { Cost } \\
========\end{array}$ & $\begin{array}{c}\text { Year } \\
=======\end{array}$ & $\begin{array}{l}\text { Discount } \\
\text { Factor } \\
=========\end{array}$ & $\begin{array}{l}\text { Discounted } \\
\text { Savings } / \text { Cost } \\
===========\end{array}$ \\
\hline Baseline Maint & $-\$ 203,964$ & Annual & 13.47 & $-\$ 2,747,395$ \\
\hline ANNUAL TOTAL & $-\$ 203,964$ & & & $-\$ 2,747,395$ \\
\hline Maintenance & $-\$ 67,736$ & 2 & .92 & $-\$ 62,505$ \\
\hline Maintenance & $-\$ 63,188$ & 3 & .89 & $-\$ 56,012$ \\
\hline Maintenance & $-\$ 223,800$ & 4 & .85 & $-\$ 190,571$ \\
\hline Maintenance & $-\$ 61,832$ & 5 & .82 & $-\$ 50,578$ \\
\hline Maintenance & $-\$ 66,896$ & 6 & .79 & $-\$ 52,565$ \\
\hline Maintenance & $-\$ 222,168$ & 7 & .75 & $-\$ 167,697$ \\
\hline Maintenance & $-\$ 71,992$ & 8 & .73 & $-\$ 52,201$ \\
\hline Maintenance & $-\$ 72,460$ & 9 & .7 & $-\$ 50,471$ \\
\hline Maintenance & $-\$ 226,556$ & 10 & .67 & $-\$ 151,589$ \\
\hline Maintenance & $-\$ 66,344$ & 11 & .64 & $-\$ 42,643$ \\
\hline Maintenance & $-\$ 72,736$ & 12 & .62 & $-\$ 44,910$ \\
\hline Maintenance & $-\$ 216,224$ & 13 & .59 & $-\$ 128,246$ \\
\hline Maintenance & $-\$ 74,108$ & 15 & .55 & $-\$ 40,561$ \\
\hline Maintenance & $-\$ 289,904$ & 16 & .53 & $-\$ 152,420$ \\
\hline Maintenance & $-\$ 76,848$ & 18 & .49 & $-\$ 37,284$ \\
\hline Maintenance & $-\$ 65,000$ & 19 & .47 & $-\$ 30,294$ \\
\hline Maintenance & $-\$ 220,480$ & 20 & .45 & $-\$ 98,709$ \\
\hline ONE TIME TOTAL & $-\$ 2,158,2$ & & & $-\$ 1,409,255$ \\
\hline TOTAL & $-\$ 2,362,2$ & & & $-\$ 4,156,651$ \\
\hline
\end{tabular}

Life Cycle Cost Analysis Study: DAVMON7.LC

Energy Conservation Investment Program (ECIP)

LCCID FY96

Installation \& Location: Davis-Monthan AFB

Region data: ARIZONA Census Region: 4

Project NO. \& Title: 250-Ton Chiller Replacement

Fiscal Year: 97 Discrete Portion: Option \#3b

Analysis Date: 10/01/97 Economic Life: 20 years

Prepared by: William T Brown III

\begin{tabular}{|c|c|c|c|c|}
\hline $\begin{array}{c}\text { Item } \\
==================\end{array}$ & $\begin{array}{l}\text { Savings/ } \\
\text { Cost } \\
=======\end{array}$ & Year & $\begin{array}{l}\text { Discount } \\
\text { Factor } \\
========\end{array}$ & $\left|\begin{array}{c}\text { Discounted } \\
\text { Savings/Cost } \\
==========\mid\end{array}\right|$ \\
\hline \multicolumn{5}{|c|}{ 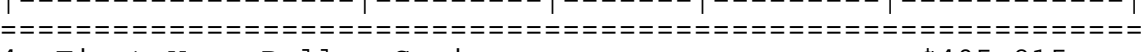 } \\
\hline \multicolumn{5}{|c|}{ 4. First Year Dollar Savings $\$ 405,815$} \\
\hline \multicolumn{4}{|c|}{ 5. Simple Payback Period (Years) } & \\
\hline \multicolumn{4}{|c|}{ 6. Total Net Discounted Savings } & 6,342 \\
\hline & \\
\hline $\begin{array}{l}\text { If }<1 \text {, Project do } \\
\text { 3. Adjusted Internal }\end{array}$ & $\begin{array}{l}\text { Des not qu } \\
\text { Rate of }\end{array}$ & & & 14.41 \\
\hline
\end{tabular}




\title{
Appendix S: Calculated Paybacks and Savings-to-Investment Ratio for Option 3c
}

\begin{abstract}
Life Cycle Cost Analysis
Installation \& Location: Davis-Monthan AFB

Region data: ARIZONA Census Region: 4

Project NO. \& Title: 250-Ton Chiller Replacement

Fiscal Year: 97 Discrete Portion: Option \#3c

Analysis Date: 10/01/97 Economic Life: 20 years

Prepared by: William $\mathrm{T}$ Brown III
\end{abstract}

ECIP Summary Report

1. Investment
A. Construction Cost
B. SIOH
C. Design Cost
D. Total Cost $(1 \mathrm{~A}+1 \mathrm{~B}+1 \mathrm{C})$
E. Salvage Value of Existing Equip.
F. Public Utility Company Rebate
G. Total Investment (1D-1E-1F)

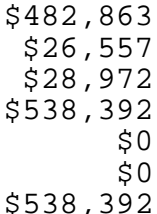

LCCID FY96

2. Energy Savings (+) / Costs (-)

Date of NISTIR 85-3273-X used for Discount Factors Oct 1995

\begin{tabular}{|c|c|c|c|c|c|c|c|}
\hline Fuel & Price & $\begin{array}{l}\text { Price } \\
\text { Units }\end{array}$ & $\begin{array}{l}\text { Usage } \\
\text { Savings }\end{array}$ & $\begin{array}{l}\text { Usage } \\
\text { Units }\end{array}$ & $\begin{array}{r}\text { Annual } \\
\text { Savings }\end{array}$ & $\begin{array}{l}\text { Discount } \\
\text { Factor }\end{array}$ & $\begin{array}{l}\text { Discounted } \\
\text { Savings }\end{array}$ \\
\hline $\begin{array}{l}==== \\
\text { Flec }\end{array}$ & $===$ & $\begin{array}{l}===== \\
\text { /Mwatt }\end{array}$ & $\begin{array}{l}======= \\
20546\end{array}$ & $=====$ & $\begin{array}{c}======== \\
5945136\end{array}$ & $=======$ & $\$ 11^{2}+{ }^{2}$ \\
\hline $\begin{array}{l}\text { Electricity } \\
\text { Elec. Deman }\end{array}$ & $\$ 46$. & Fiwale & 20,340 & Holl & $\$ 23$ & & $\$ 3,125,795$ \\
\hline $\begin{array}{l}\text { Natural Gas } \\
\text { TOTAL }\end{array}$ & $\$ 3.4$ & /Mbtus & $\begin{array}{l}-179,077 \\
-108,970\end{array}$ & $\begin{array}{l}\text { Mbtus } \\
\text { Mbtus }\end{array}$ & $\begin{array}{r}-\$ 608,863 \\
\$ 568,329\end{array}$ & 17.32 & $\begin{array}{r}-\$ 10,545,51 \\
\$ 6,256,406\end{array}$ \\
\hline
\end{tabular}


3. Non Energy Savings (+) / Costs (-)

\begin{tabular}{|c|c|c|c|c|}
\hline 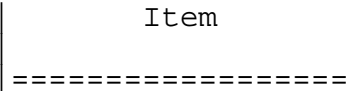 & $\begin{array}{l}\text { Savings / } \\
\text { Cost } \\
========\end{array}$ & $======$ & $\begin{array}{l}\text { Discount } \\
\text { Factor } \\
========\end{array}$ & $\begin{array}{l}\text { Discounted } \\
\text { Savings / Cost } \\
==========\end{array}$ \\
\hline Baseline Maint & $-\$ 152,973$ & Annual & 13.47 & $-\$ 2,060,546$ \\
\hline ANNUAL TOTAL & $-\$ 152,973$ & & & $-\$ 2,060,546$ \\
\hline Maintenance & $-\$ 50,802$ & 2 & .92 & $-\$ 46,879$ \\
\hline Maintenance & $-\$ 47,391$ & 3 & .89 & $-\$ 42,009$ \\
\hline Maintenance & $-\$ 167,850$ & 4 & .85 & $-\$ 142,928$ \\
\hline Maintenance & $-\$ 46,374$ & 5 & .82 & $-\$ 37,933$ \\
\hline Maintenance & $-\$ 50,172$ & 6 & .79 & $-\$ 39,424$ \\
\hline Maintenance & $-\$ 166,626$ & 7 & .75 & $-\$ 125,773$ \\
\hline Maintenance & 3,994 & 8 & .73 & $-\$ 39,151$ \\
\hline Maintenance &, 345 & 9 & .7 & $-\$ 37,853$ \\
\hline Maintenance & 9,917 & 10 & .67 & $-\$ 113,692$ \\
\hline Maintenance & $-\$ 49,758$ & 11 & .64 & $-\$ 31,982$ \\
\hline Maintenance & $-\$ 54,552$ & 12 & .62 & $-\$ 33,682$ \\
\hline Mainten & 2,168 & 13 & .59 & $-\$ 96,185$ \\
\hline Maintenance &, 581 & 15 & .55 & $-\$ 30,420$ \\
\hline Maintenance &, 428 & 16 & .53 & $-\$ 114,315$ \\
\hline Maintenance & 7,636 & 18 & .49 & $-\$ 27,963$ \\
\hline Maintenance & $-\$ 48,750$ & 19 & .47 & $-\$ 22,720$ \\
\hline Maintenance & $-\$ 165,360$ & 20 & .45 & $-\$ 74,031$ \\
\hline ONE TIME TOTAL & $-\$ 1,618,7$ & & & $-\$ 1,056,941$ \\
\hline TOTAL & $-\$ 1,771,6$ & & & $-\$ 3,117,488$ \\
\hline
\end{tabular}

Life Cycle Cost Analysis Study: DAVMON8.LC

Energy Conservation Investment Program (ECIP)

LCCID FY96

Installation \& Location: Davis-Monthan AFB

Region data: ARIZONA Census Region: 4

Project NO. \& Title: 250-Ton Chiller Replacement

Fiscal Year: 97 Discrete Portion: Option \#3c

Analysis Date: 10/01/97 Economic Life: 20 years

Prepared by: William $\mathrm{T}$ Brown III

\begin{tabular}{|c|c|c|c|c|}
\hline $\begin{array}{c}\text { Item } \\
==================\end{array}$ & $\left|\begin{array}{l}\text { Savings } / \\
\text { Cost } \\
========\end{array}\right|$ & $=======$ & $\begin{array}{l}\text { Discount } \\
\text { Factor } \\
========\end{array}$ & $\mid \begin{array}{c}\text { Discounted } \\
\text { Savings /Cost } \\
==========\end{array}$ \\
\hline \multicolumn{5}{|c|}{$=$} \\
\hline \multicolumn{5}{|c|}{$\begin{array}{lc}\text { 4. First Year Dollar Savings } & \$ 334,421 \\
\text { 5. Simple Payback Period (Years) } & 1.49\end{array}$} \\
\hline \multicolumn{5}{|c|}{$\begin{array}{l}\text { 5. Simple Payback Period (Years) } \\
\text { 6. Total Net Discounted Savings }\end{array}$} \\
\hline \multicolumn{4}{|c|}{ 6. Total Net Discounted Savings } & $\$ 3,138,918$ \\
\hline \multicolumn{5}{|c|}{ 7. Savings to Investment Ratio } \\
\hline 8. Adjusted Internal & & & & $13.69 \%$ \\
\hline
\end{tabular}




\section{Appendix T: Calculated Paybacks and Savings-to-Investment Ratio for Option 3d}

\begin{abstract}
Life Cycle Cost Analysis Study: Option \#3d Energy Conservation Investment Program (ECIP) Installation \& Location: Davis-Monthan AFB Region data: ARIZONA Census Region: 4 Project NO. \& Title: 250-Ton Chiller Replacement Fiscal Year: 97 Discrete Portion: Option \#3d

Analysis Date: 10/01/97 Economic Life: 20 years Prepared by: William $\mathrm{T}$ Brown III
\end{abstract}

LCCID FY96

\section{ECIP Summary Report}

1. Investment
A. Construction Cost
B. $\mathrm{SIOH}$
C. Design Cost
D. Total Cost $(1 A+1 B+1 C)$
E. Salvage Value of Existing Equip.
F. Public Utility Company Rebate
G. Total Investment (1D-1E-1F)

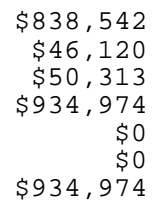

2. Energy Savings (+) / Costs (-) Date of NISTIR 85-3273-X used for Discount Factors Oct 1995

\begin{tabular}{|c|c|c|c|c|c|c|c|}
\hline Fuel & Price & $\begin{array}{l}\text { Price } \\
\text { Units } \\
=====\end{array}$ & $\begin{array}{l}\text { Usage } \\
\text { Savings } \\
=======\end{array}$ & $\begin{array}{l}\text { Usage } \\
\text { Units } \\
======\end{array}$ & $\begin{array}{c}\text { Annual } \\
\text { Savings } \\
=========\end{array}$ & $\begin{array}{l}\text { Discount } \\
\text { Factor } \\
=======\end{array}$ & $\begin{array}{l}\text { Discounted } \\
\text { Savings } \\
==========\end{array}$ \\
\hline Electricity & $\$ 46$. & /Mwatt & 40,791 & Mwatt- & $\$ 1,876,397$ & $\begin{array}{l}14.47 \\
13.47\end{array}$ & $\$ 27,151,460$ \\
\hline $\begin{array}{l}\text { Noc. Deman } \\
\text { Natural Gas } \\
\text { TOTAL }\end{array}$ & $\$ 3.4$ & /Mbtus & $\begin{array}{l}-376,046 \\
-236,860\end{array}$ & $\begin{array}{l}\text { Mbtus } \\
\text { Mbtus }\end{array}$ & $\begin{array}{r}\$ 1,278,55 \\
-\$ 1,066,622\end{array}$ & $\begin{array}{l}13.41 \\
17.32\end{array}$ & $\begin{array}{l}\$ 0, \$ 22,144,57 \\
-\$ 11,321,360\end{array}$ \\
\hline
\end{tabular}


3. Non Energy Savings (+) / Costs (-)

\begin{tabular}{|c|c|c|c|c|}
\hline 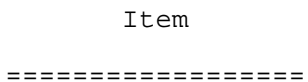 & $\begin{array}{l}\text { Savings/ } \\
\text { Cost } \\
========\end{array}$ & $=======$ & $\begin{array}{l}\text { Discount } \\
\text { Factor } \\
=========\end{array}$ & $\begin{array}{l}\text { Discounted } \\
\text { Savings/Cost } \\
===========\end{array}$ \\
\hline Baseline Maint & $-\$ 305,946$ & Annual & 13.47 & $-\$ 4,121,093$ \\
\hline ANNUAL TOTAL & $-\$ 305,946$ & & & $-\$ 4,121,093$ \\
\hline Maintenance & $-\$ 101,604$ & 2 & .92 & $-\$ 93,758$ \\
\hline Maintenance & $-\$ 94,782$ & 3 & .89 & $-\$ 84,018$ \\
\hline Maintenance & $-\$ 335,700$ & 4 & .85 & $-\$ 285,857$ \\
\hline Maintenance & $-\$ 92,748$ & 5 & .82 & $-\$ 75,867$ \\
\hline Maintenance & $-\$ 100,344$ & 6 & .79 & $-\$ 78,847$ \\
\hline Maintenance & $-\$ 333,252$ & 7 & .75 & $-\$ 251,546$ \\
\hline Maintenance & $-\$ 107,988$ & 8 & .73 & $-\$ 78,301$ \\
\hline Maintenance & $-\$ 108,690$ & 9 & .7 & $-\$ 75,706$ \\
\hline Maintenance & $-\$ 339,834$ & 10 & .67 & $-\$ 227,384$ \\
\hline Maintenance & $-\$ 99,516$ & 11 & .64 & $-\$ 63,964$ \\
\hline Maintenance & $-\$ 109,104$ & 12 & .62 & $-\$ 67,365$ \\
\hline Maintenance & $-\$ 324,336$ & 13 & .59 & $-\$ 192,369$ \\
\hline Maintenance & $-\$ 111,162$ & 15 & .55 & $-\$ 60,841$ \\
\hline Maintenance & $-\$ 434,856$ & 16 & .53 & $-\$ 228,630$ \\
\hline Maintenance & $-\$ 115,272$ & 18 & .49 & $-\$ 55,926$ \\
\hline Maintenance & $-\$ 97,500$ & 19 & .47 & $-\$ 45,440$ \\
\hline Maintenance & $-\$ 330,720$ & 20 & .45 & $-\$ 148,063$ \\
\hline ONE TIME TOTAL & $-\$ 3,237,4$ & & & $-\$ 2,113,883$ \\
\hline TOTAL & $-\$ 3,543,3$ & & & $-\$ 6,234,975$ \\
\hline
\end{tabular}

Life Cycle Cost Analysis

Study: DAVMON9.LC

Energy Conservation Investment Program (ECIP)

Installation \& Location: Davis-Monthan AFB
Region data: ARIZONA

Project NO. \& Title: 250-Ton Chiller Replacement

Fiscal Year: 97 Discrete Portion: Option \#3d

Analysis Date: 10/01/97 Economic Life: 20 years

Prepared by: William T Brown III

\begin{tabular}{|c|c|c|c|c|}
\hline$==0==$ & $\left|\begin{array}{l}\text { Savings } / \\
\text { Cost } \\
========\mid\end{array}\right|$ & & $\begin{array}{l}\text { Discount } \\
\text { Factor } \\
=======\end{array}$ & $\left|\begin{array}{c}\text { Discounted } \\
\text { Savings } / \text { Cost } \\
==========\end{array}\right|$ \\
\hline \multicolumn{5}{|c|}{ =" } \\
\hline \multicolumn{5}{|c|}{$\begin{array}{lc}\text { 4. First Year Dollar Savings } & \$ 598,806 \\
\text { 5. Simple Payback Period (Years) } & 1.43 \\
\text { 6. Total Net Discounted Savings } & \$ 5,086,382 \\
\text { 7. Savings to Investment Ratio } & 5.44 \\
\text { If < 1, Project does not qualify } & \end{array}$} \\
\hline - Adjusted Interna & & & & \\
\hline
\end{tabular}


